Supporting Information for:

\title{
Limonoids from Cipadessa baccifera
}

Jin-Hai Yu, ${ }^{\dagger}$ Hua Zhang, ${ }^{\dagger}$ Bin Zhou, ${ }^{\dagger}$ Flavia M. Zimbres, ${ }^{\#}$ Seema Dalal, ${ }^{\dagger}$ Qun-Fang Liu, ${ }^{\dagger}$ Maria B. Cassera, ${ }^{\#}$ and Jian-Min Yue ${ }^{* \dagger}$

†State Key Laboratory of Drug Research, Shanghai Institute of Materia Medica, Chinese Academy of Sciences, 555 Zu Chong Zhi Road, Shanghai, 201203, People's Republic of China

\#Department of Biochemistry and Molecular Biology, and Center for Tropical and Emerging Global Diseases (CTEGD), University of Georgia, Athens, Georgia 30602, United States

${ }^{ \pm}$Department of Biochemistry, MC 0308, Virginia Polytechnic Institute and State University, Blacksburg, Virginia 24061, United States 


\section{Table of Contents}

Table S1. Crystal data and structure refinement for 3a.

Table S2. Comparison of experimental ${ }^{13} \mathrm{C}$ NMR data with calculated ones of compounds 8, 9, 11 and 18.

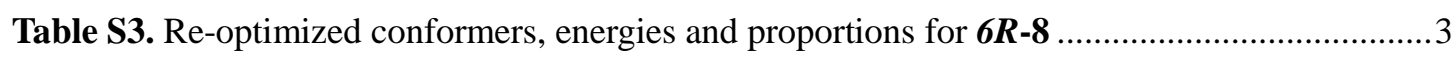

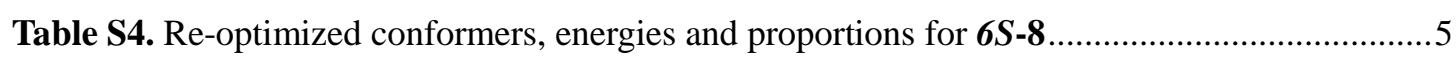

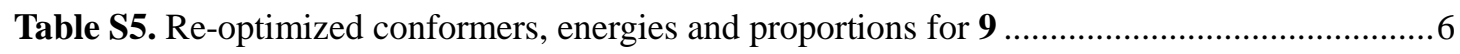

Table S6. Re-optimized conformers, energies and proportions for 11...........................................

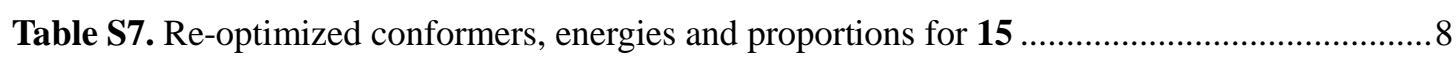

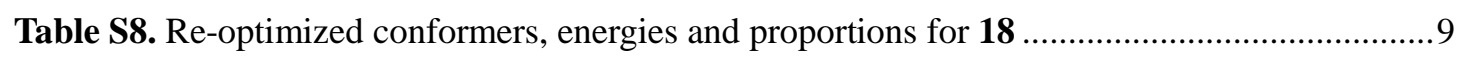

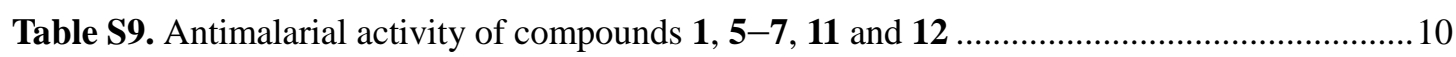

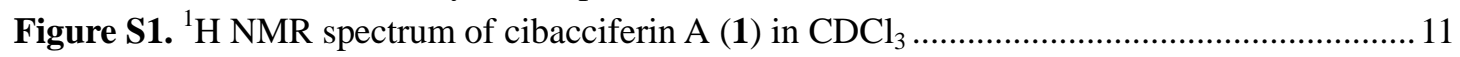

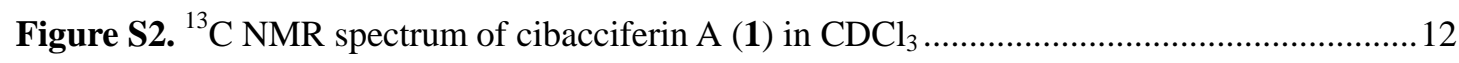

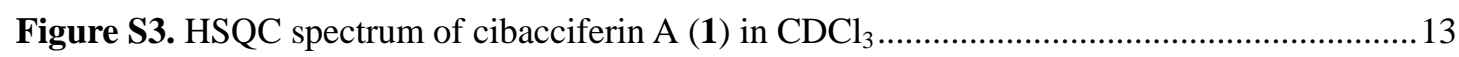

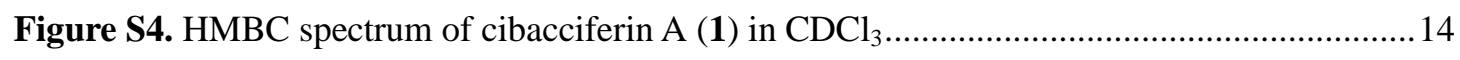

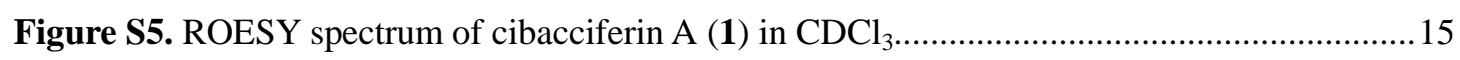

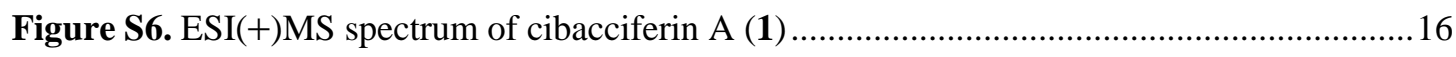

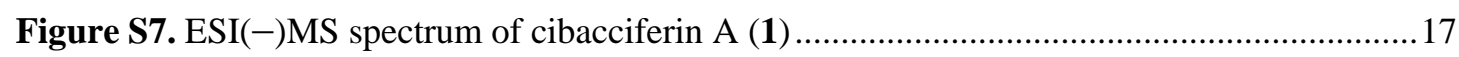

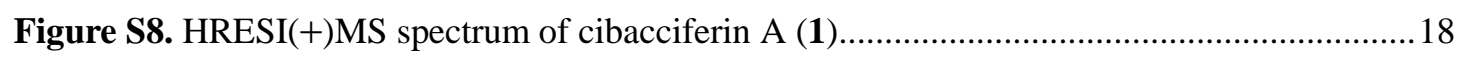

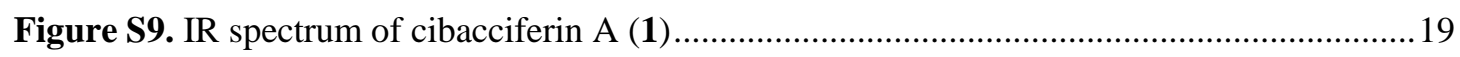

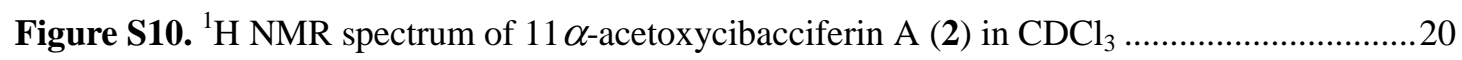

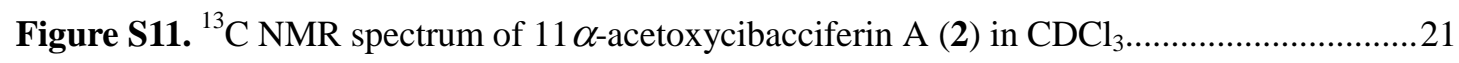

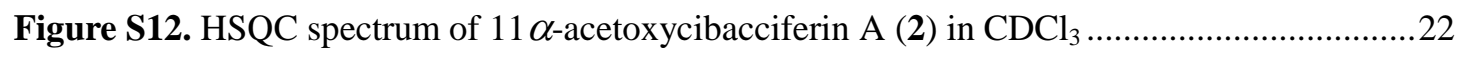

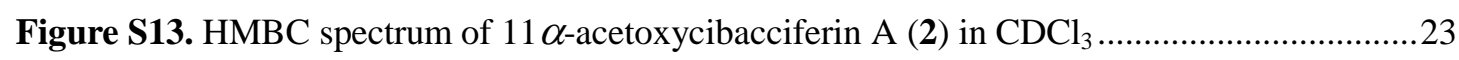

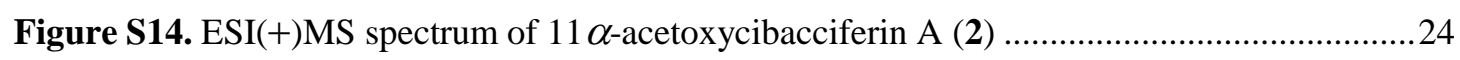

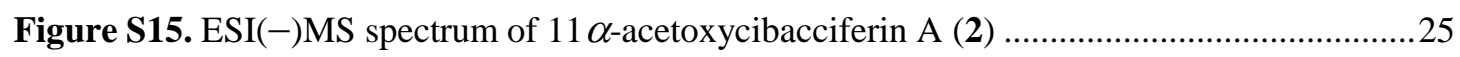

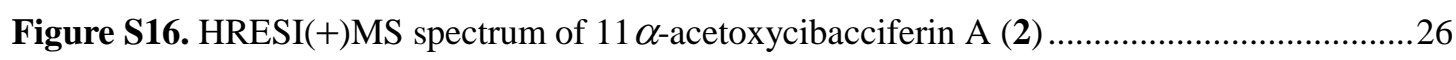

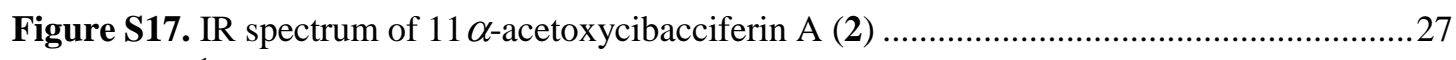

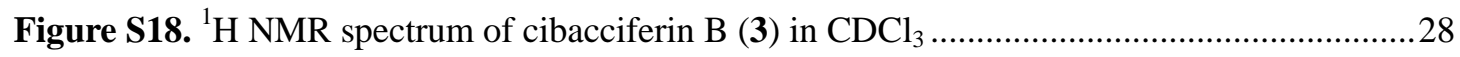

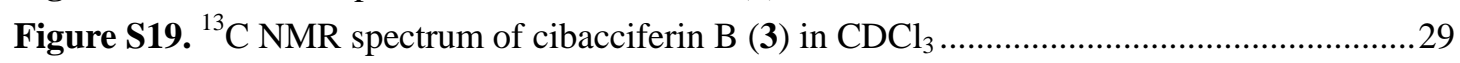

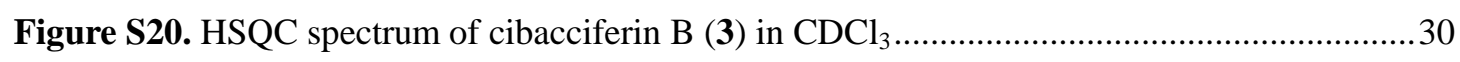

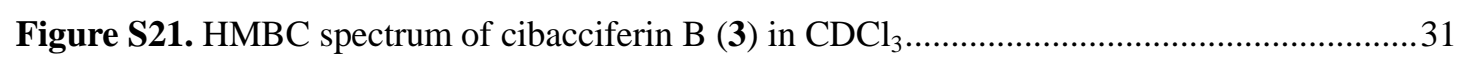

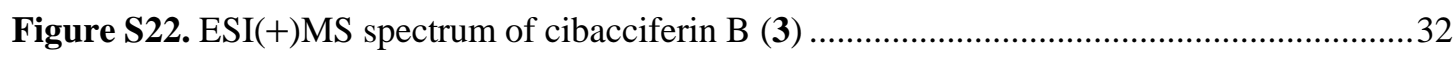

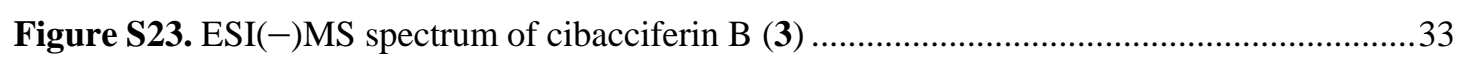

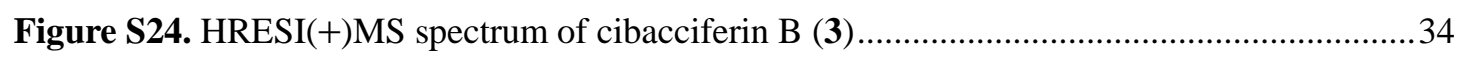

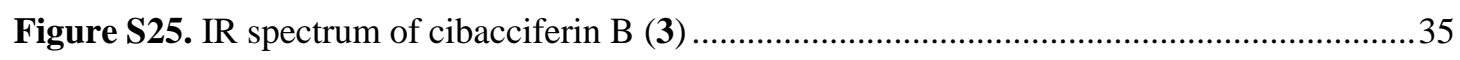

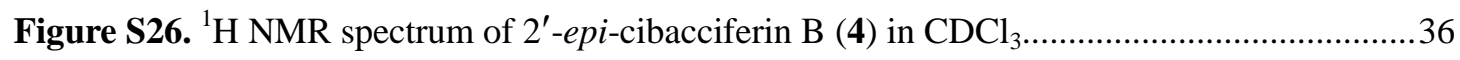

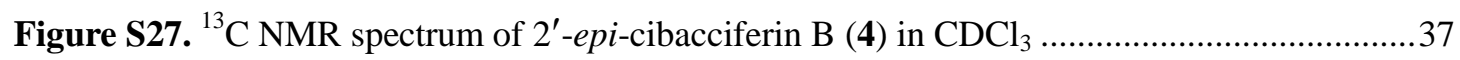

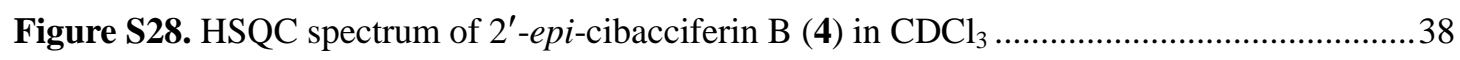

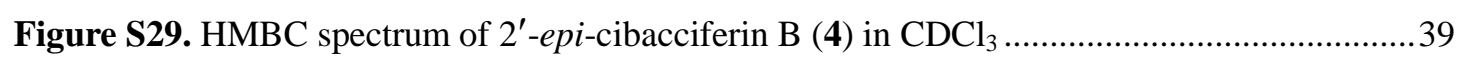

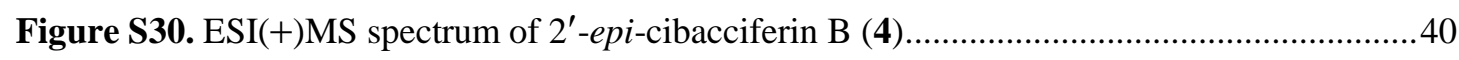

Figure S31. ESI(-)MS spectrum of 2'-epi-cibacciferin B (4)................................................... 41

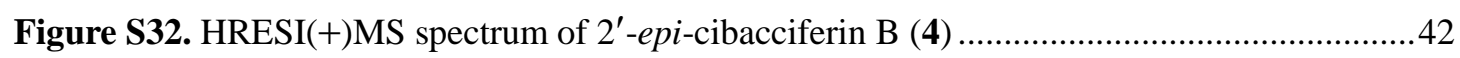


Figure S33. IR spectrum of $2^{\prime}$-epi-cibacciferin B (4)

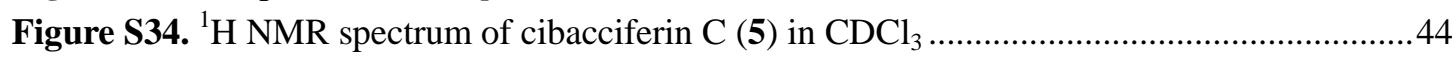

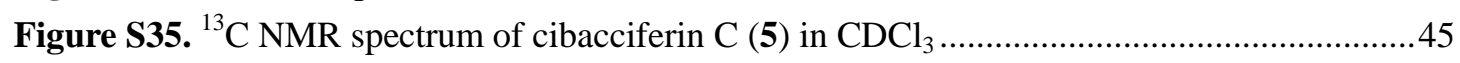

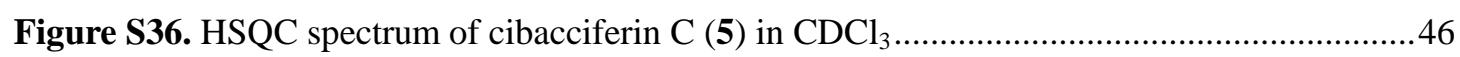

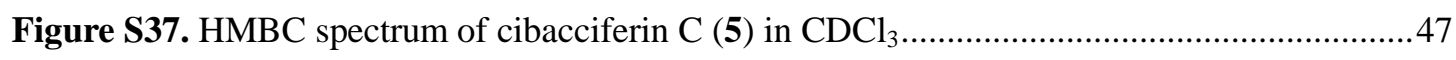

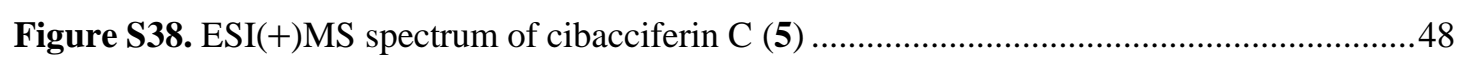

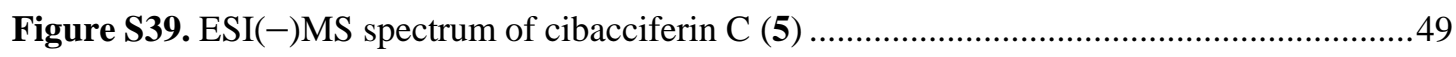

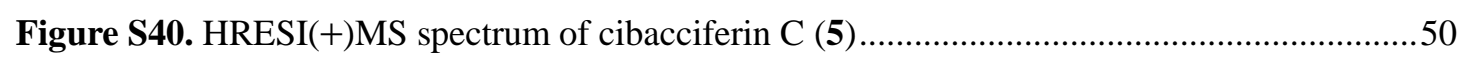

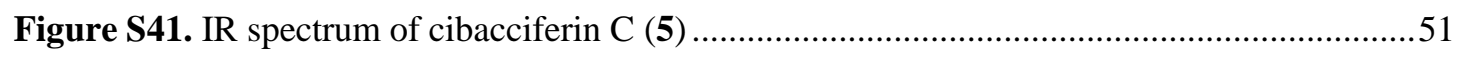

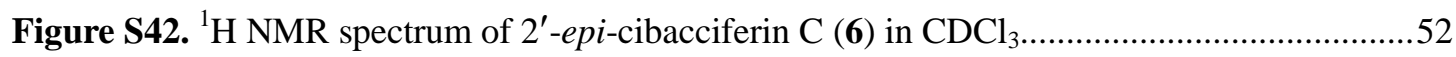

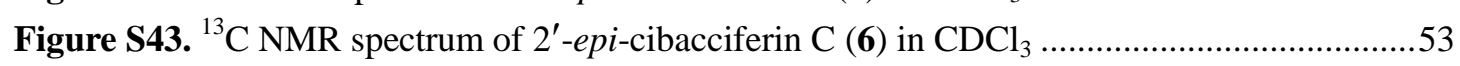

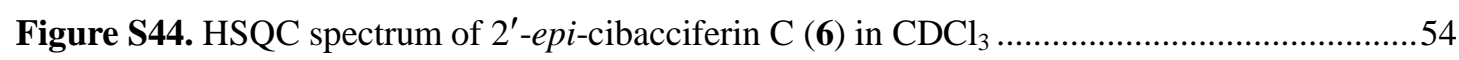

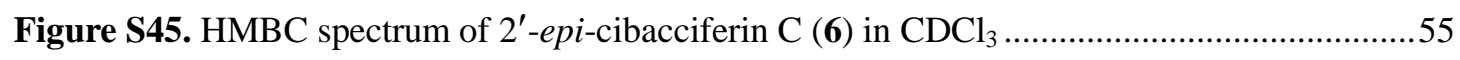

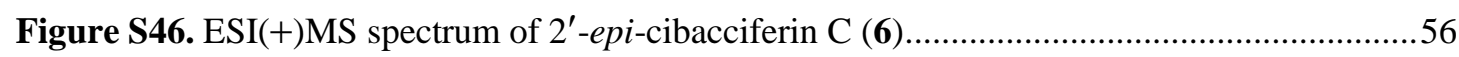

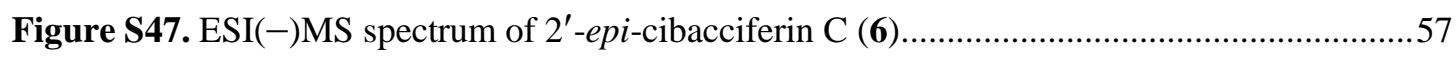

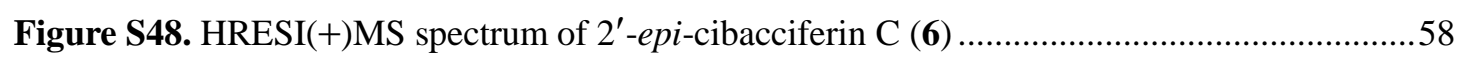

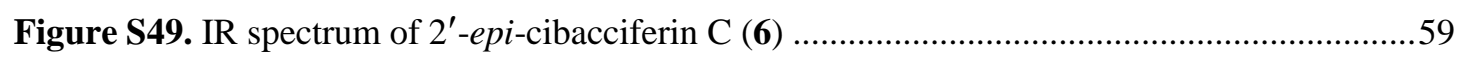

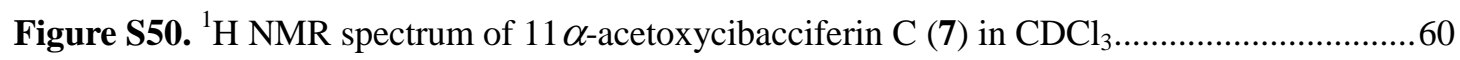

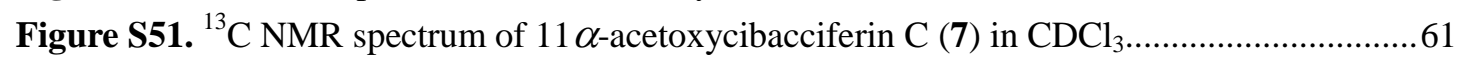

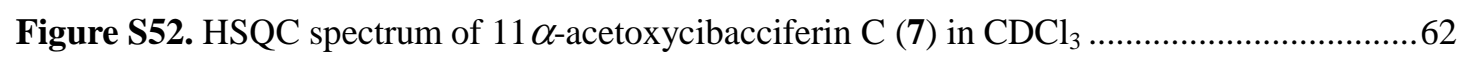

Figure S53. HMBC spectrum of $11 \alpha$-acetoxycibacciferin $\mathrm{C}(7)$ in $\mathrm{CDCl}_{3} \ldots \ldots \ldots \ldots \ldots \ldots \ldots \ldots \ldots . . . . . . . . . . . . . . . . . . .63$

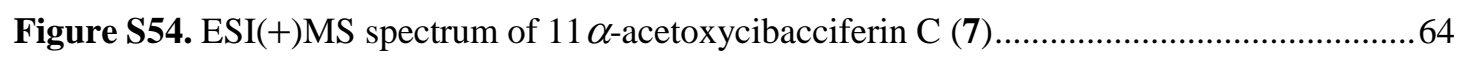

Figure S55. ESI(-)MS spectrum of $11 \alpha$-acetoxycibacciferin C (7)......................................65

Figure S56. HRESI(+)MS spectrum of $11 \alpha$-acetoxycibacciferin C (7) ....................................66

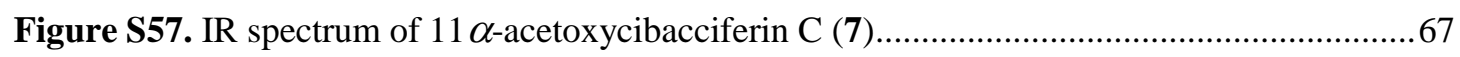

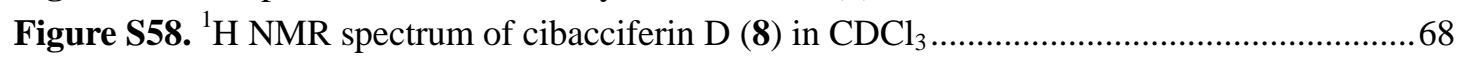

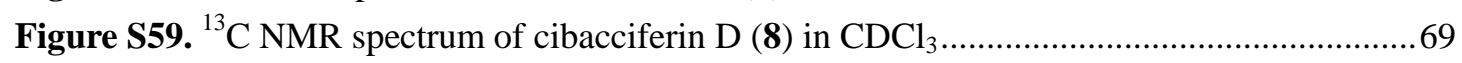

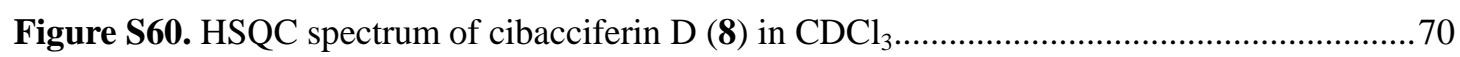

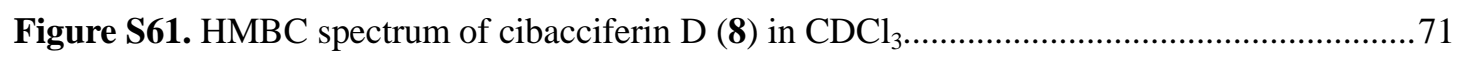

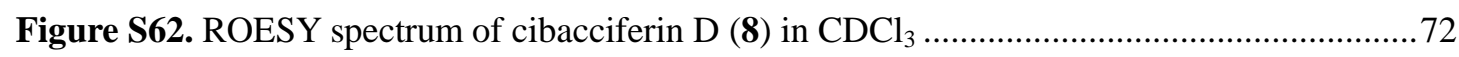

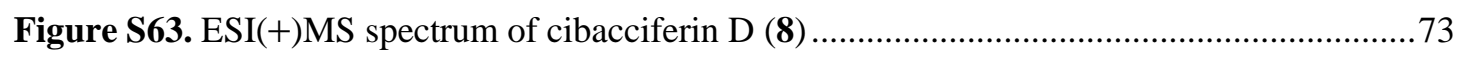

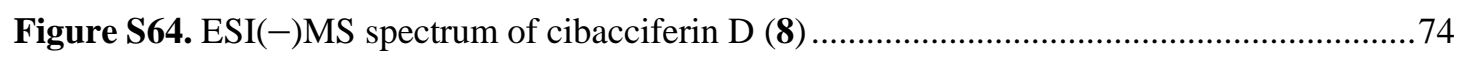

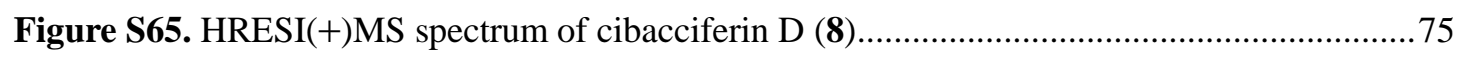

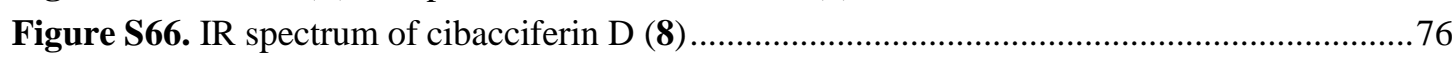

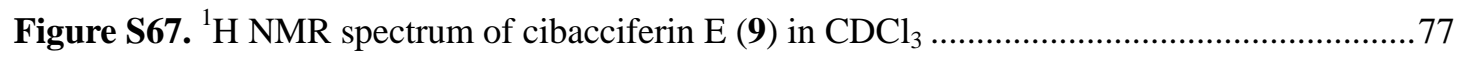

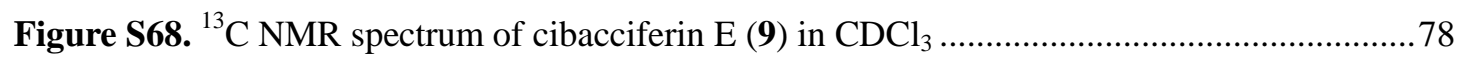

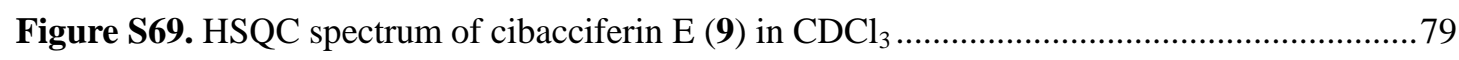

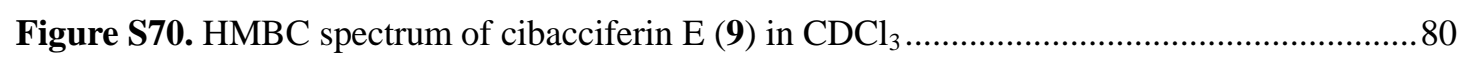

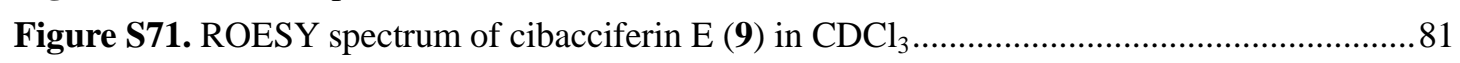

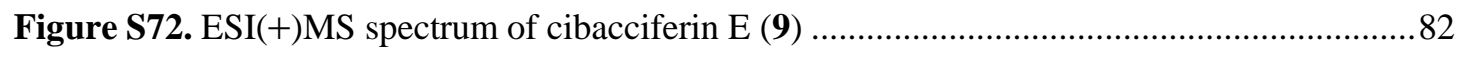

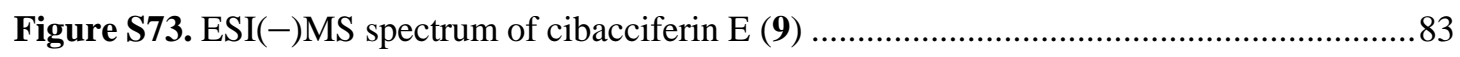

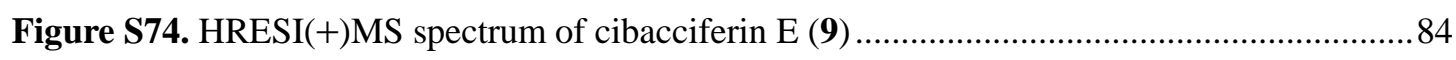

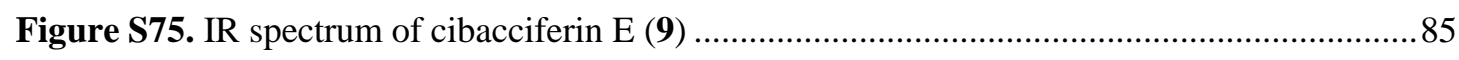

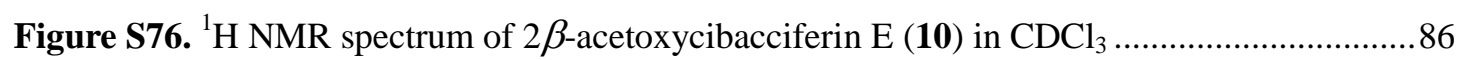




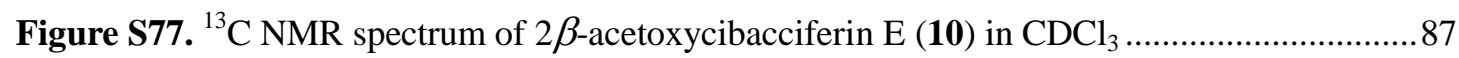

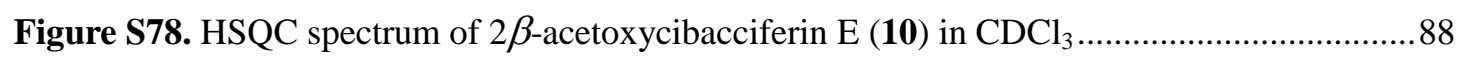

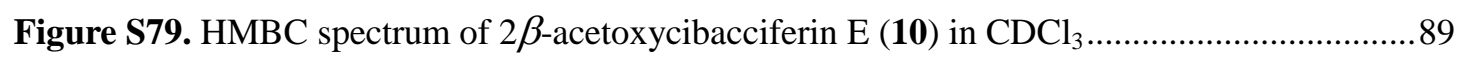

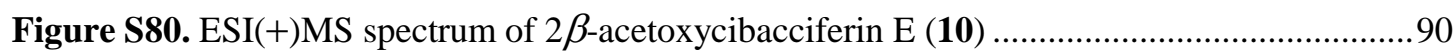

Figure S81. ESI(-)MS spectrum of $2 \beta$-acetoxycibacciferin E (10) ..........................................

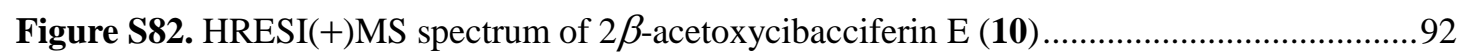

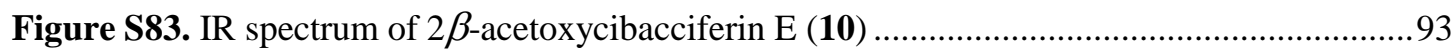

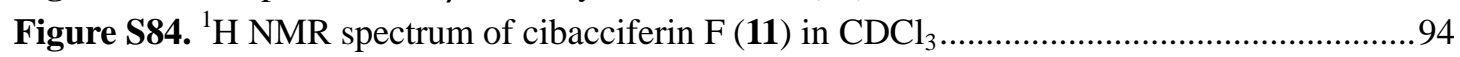

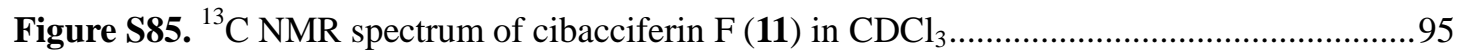

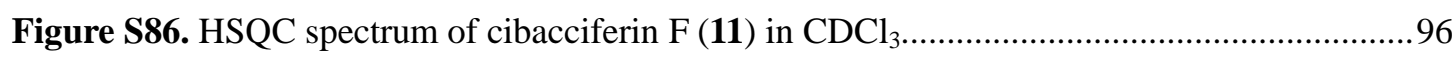

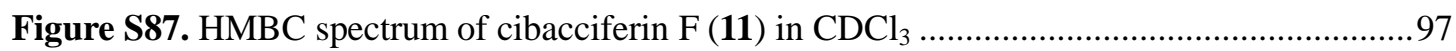

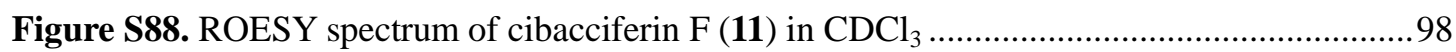

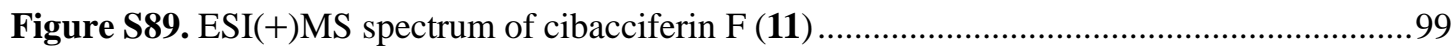

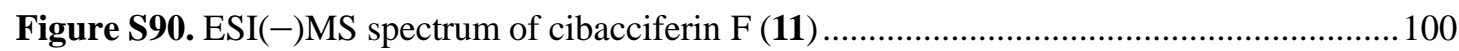

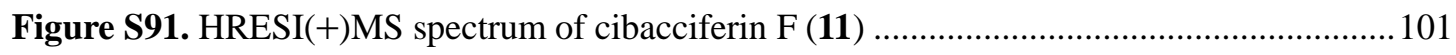

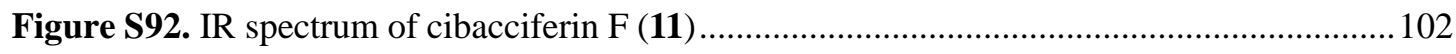

Figure S93. ${ }^{1} \mathrm{H}$ NMR spectrum of 6-dehydroxycibacciferin $\mathrm{F}(\mathbf{1 2})$ in $\mathrm{CDCl}_{3} \ldots \ldots \ldots \ldots \ldots \ldots \ldots \ldots \ldots . . . . . . . . . . . .103$

Figure S94. ${ }^{13} \mathrm{C}$ NMR spectrum of 6-dehydroxycibacciferin $\mathrm{F}(\mathbf{1 2})$ in $\mathrm{CDCl}_{3} \ldots \ldots \ldots \ldots \ldots \ldots \ldots \ldots \ldots . . . . . . . . . . . . . .104$

Figure S95. HSQC spectrum of 6-dehydroxycibacciferin $\mathrm{F}(\mathbf{1 2})$ in $\mathrm{CDCl}_{3} \ldots \ldots \ldots \ldots \ldots \ldots \ldots \ldots \ldots \ldots . . . . . . . . . . . . . .105$

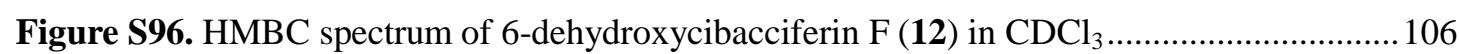

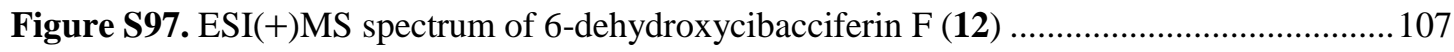

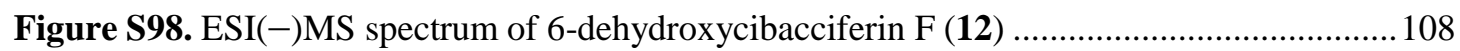

Figure S99. HRESI(+)MS spectrum of 6-dehydroxycibacciferin F (12) .................................109

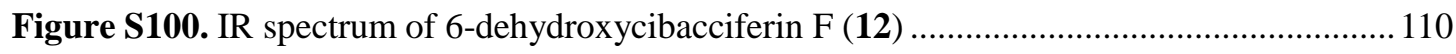

Figure S101. ${ }^{1} \mathrm{H}$ NMR spectrum of 12-deacetoxycibacciferin E (13) in $\mathrm{CDCl}_{3} \ldots \ldots \ldots \ldots \ldots \ldots \ldots \ldots . . . . . . . . . .111$

Figure S102. ${ }^{13} \mathrm{C}$ NMR spectrum of 12-deacetoxycibacciferin $\mathrm{E}(\mathbf{1 3})$ in $\mathrm{CDCl}_{3}$...........................112

Figure S103. HSQC spectrum of 12-deacetoxycibacciferin $\mathrm{E}(\mathbf{1 3})$ in $\mathrm{CDCl}_{3} \ldots \ldots \ldots \ldots \ldots \ldots \ldots \ldots \ldots . . . . . . . \ldots 13$

Figure S104. HMBC spectrum of 12-deacetoxycibacciferin E (13) in $\mathrm{CDCl}_{3} \ldots \ldots \ldots \ldots \ldots \ldots \ldots \ldots \ldots \ldots . . . . . . . . . . . . . .114$

Figure S105. ESI(+)MS spectrum of 12-deacetoxycibacciferin E (13) .....................................115

Figure S106. ESI(-)MS spectrum of 12-deacetoxycibacciferin E (13) ..................................116

Figure S107. HRESI(+)MS spectrum of 12-deacetoxycibacciferin E (13).................................117

Figure S108. IR spectrum of 12-deacetoxycibacciferin E (13) ................................................. 118

Figure S109. ${ }^{1} \mathrm{H}$ NMR spectrum of $2 \beta$-acetoxy-12-deacetoxycibacciferin $\mathrm{E}(\mathbf{1 4})$ in $\mathrm{CDCl}_{3} \ldots \ldots . .119$

Figure S110. ${ }^{13} \mathrm{C}$ NMR spectrum of $2 \beta$-acetoxy-12-deacetoxycibacciferin $\mathrm{E}(\mathbf{1 4})$ in $\mathrm{CDCl}_{3} \ldots . .120$

Figure S111. HSQC spectrum of $2 \beta$-acetoxy-12-deacetoxycibacciferin $\mathrm{E}(\mathbf{1 4})$ in $\mathrm{CDCl}_{3} \ldots \ldots \ldots . . .121$

Figure S112. HMBC spectrum of $2 \beta$-acetoxy-12-deacetoxycibacciferin $\mathrm{E}$ (14) in $\mathrm{CDCl}_{3} \ldots \ldots . .122$

Figure S113. ESI(+)MS spectrum of $2 \beta$-acetoxy-12-deacetoxycibacciferin E (14)..................... 123

Figure S114. ESI(-)MS spectrum of $2 \beta$-acetoxy-12-deacetoxycibacciferin E (14)..................... 124

Figure S115. HRESI(+)MS spectrum of $2 \beta$-acetoxy-12-deacetoxycibacciferin E (14) ............... 125

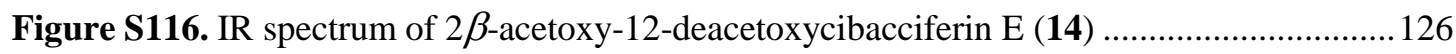

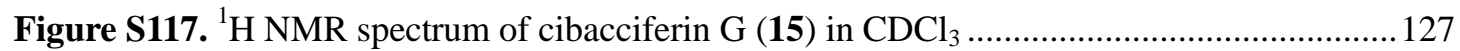

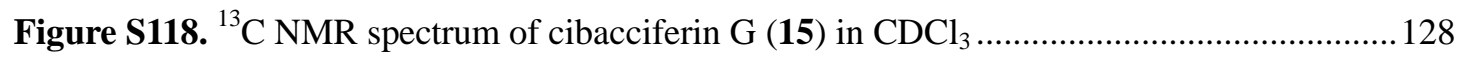

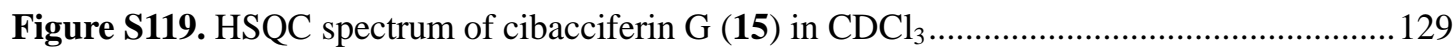

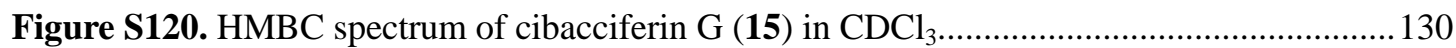




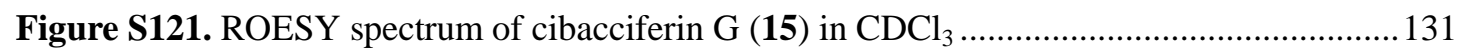

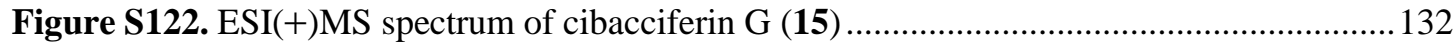

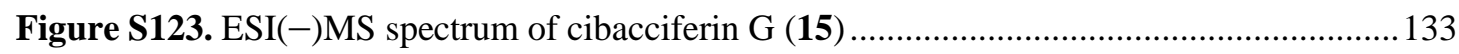

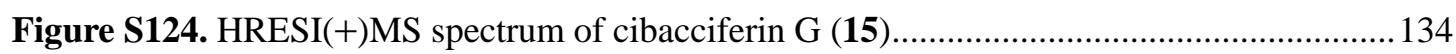

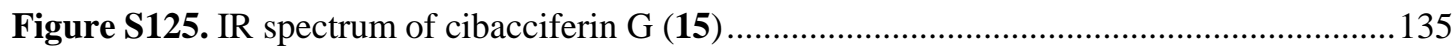

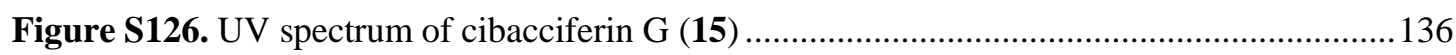

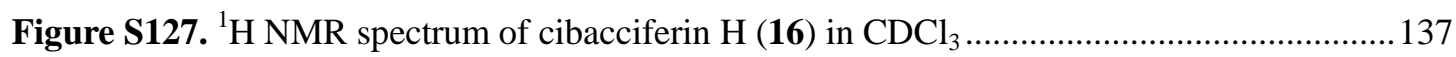

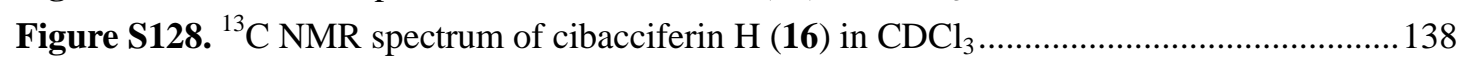

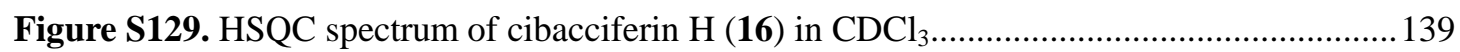

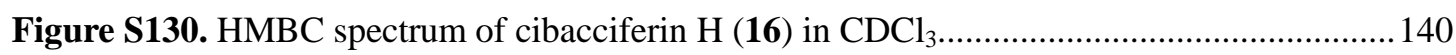

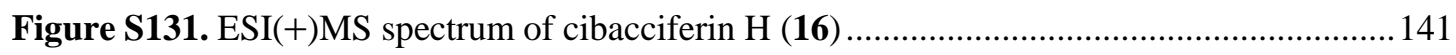

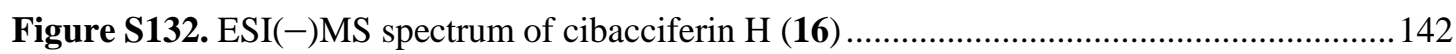

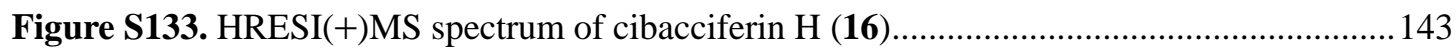

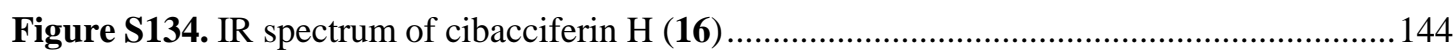

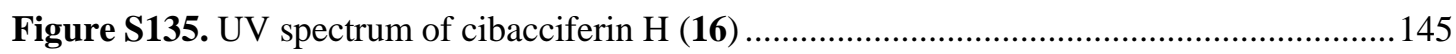

Figure S136. ${ }^{1} \mathrm{H}$ NMR spectrum of 12-dehydroxycibacciferin $\mathrm{H}(\mathbf{1 7})$ in $\mathrm{CDCl}_{3} \ldots \ldots \ldots \ldots \ldots \ldots \ldots \ldots . . . . . . . . . . . . .146$

Figure S137. ${ }^{13} \mathrm{C}$ NMR spectrum of 12-dehydroxycibacciferin $\mathrm{H}(\mathbf{1 7})$ ) in $\mathrm{CDCl}_{3} \ldots \ldots \ldots \ldots \ldots \ldots \ldots . . . .147$

Figure S138. HSQC spectrum of 12-dehydroxycibacciferin $\mathrm{H}(\mathbf{1 7})$ in $\mathrm{CDCl}_{3} \ldots \ldots \ldots \ldots \ldots \ldots \ldots \ldots . . . . . . . . . . . . .148$

Figure S139. HMBC spectrum of 12-dehydroxycibacciferin $\mathrm{H}(\mathbf{1 7})$ in $\mathrm{CDCl}_{3} \ldots \ldots \ldots \ldots \ldots \ldots \ldots \ldots \ldots . . . . . . . . . . . .149$

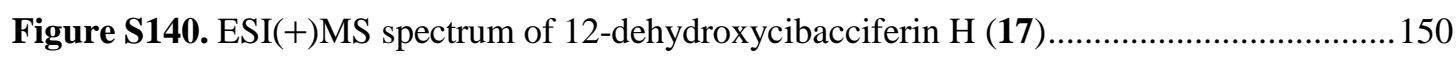

Figure S141. ESI(-)MS spectrum of 12-dehydroxycibacciferin H (17)...................................151

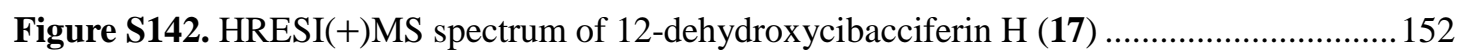

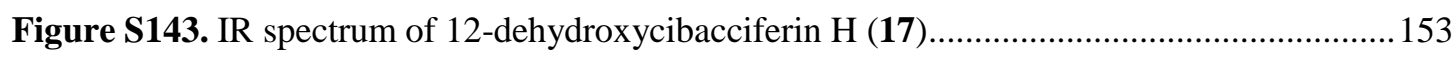

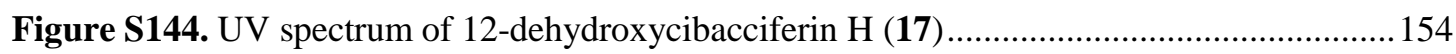

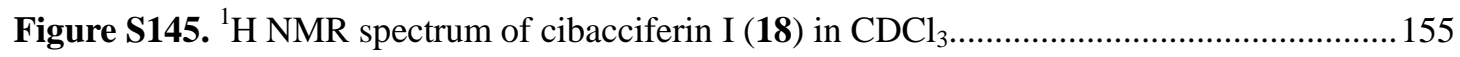

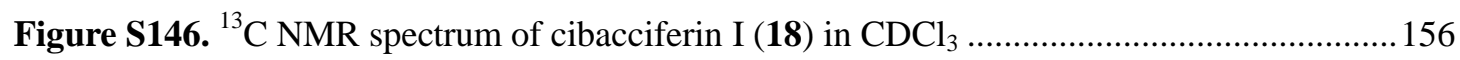

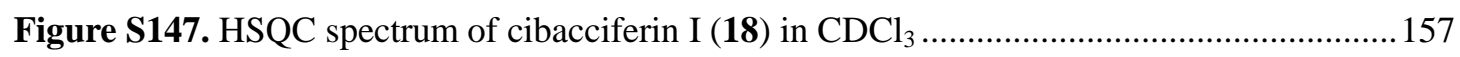

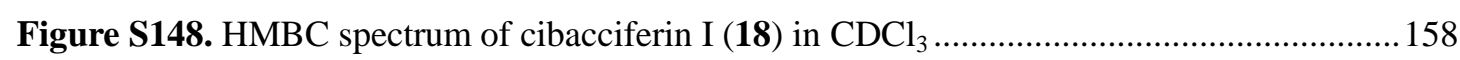

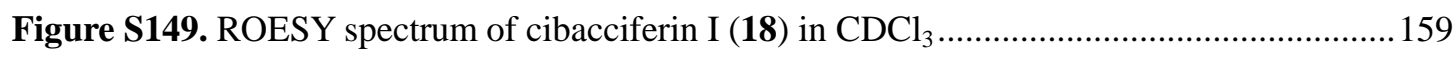

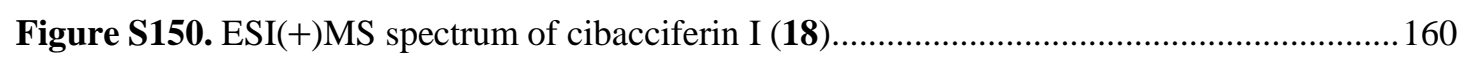

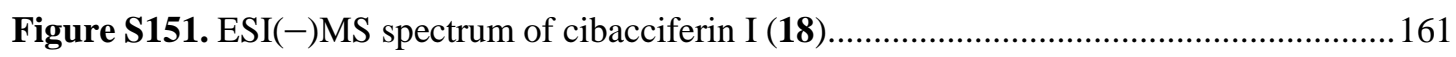

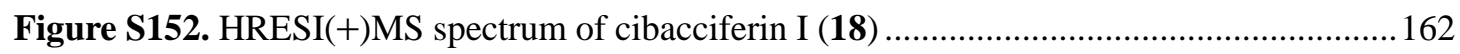

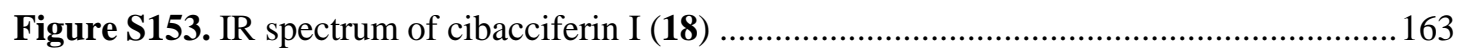

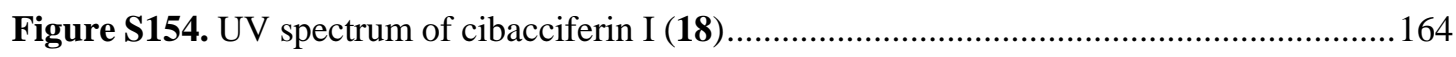

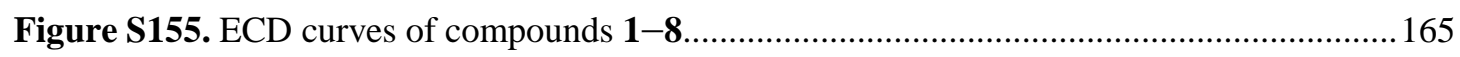

Figure S156. ECD curves of compounds 9-14

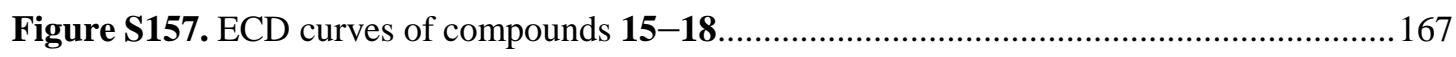

Figure S158. Comparison of the experimental ${ }^{13} \mathrm{C}$ NMR data of compound 9 with the calculated

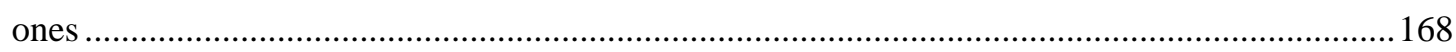

Figure S159. Comparison of the experimental ${ }^{13} \mathrm{C}$ NMR data of compound 11 with the calculated

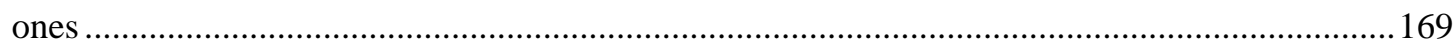

Figure S160. Comparison of the experimental ${ }^{13} \mathrm{C}$ NMR data of compound 18 with the calculated ones 
Table S1. Crystal data and structure refinement for 3a

\begin{tabular}{|c|c|c|}
\hline Empirical formula & \multicolumn{2}{|l|}{$\mathrm{C}_{32} \mathrm{H}_{44} \mathrm{O}_{10}$} \\
\hline Formula weight & \multicolumn{2}{|l|}{588.67} \\
\hline Temperature & \multicolumn{2}{|l|}{$296.15 \mathrm{~K}$} \\
\hline Wavelength & \multicolumn{2}{|l|}{$1.54178 \AA$} \\
\hline Crystal system & \multicolumn{2}{|l|}{ Orthorhombic } \\
\hline Space group & \multicolumn{2}{|l|}{ P 212121} \\
\hline \multirow{3}{*}{ Unit cell dimensions } & $\mathrm{a}=8.49070(10) \AA$ & $\alpha=90^{\circ}$ \\
\hline & $\mathrm{b}=9.26300(10) \AA$ & $\beta=90^{\circ}$ \\
\hline & $\mathrm{c}=38.6476(6) \AA$ & $\gamma=90^{\circ}$ \\
\hline Volume & \multicolumn{2}{|l|}{$3039.61(7) \AA^{3}$} \\
\hline $\mathrm{Z}$ & \multicolumn{2}{|l|}{4} \\
\hline Density (calculated) & \multicolumn{2}{|l|}{$1.286 \mathrm{Mg} / \mathrm{m}^{3}$} \\
\hline Absorption coefficient & \multicolumn{2}{|l|}{$0.782 \mathrm{~mm}^{-1}$} \\
\hline $\mathrm{F}(000)$ & \multicolumn{2}{|l|}{1264} \\
\hline Crystal size & \multicolumn{2}{|c|}{$0.2 \times 0.15 \times 0.12 \mathrm{~mm}^{3}$} \\
\hline Theta range for data collection & \multicolumn{2}{|l|}{2.286 to $69.680^{\circ}$} \\
\hline Index ranges & \multicolumn{2}{|c|}{$-10<=\mathrm{h}<=10,-10<=\mathrm{k}<=7,-46<=1<=46$} \\
\hline Reflections collected & \multicolumn{2}{|l|}{20035} \\
\hline Independent reflections & \multicolumn{2}{|c|}{$5399[\mathrm{R}(\mathrm{int})=0.0291]$} \\
\hline Completeness to theta $=67.679^{\circ}$ & \multicolumn{2}{|l|}{$96.9 \%$} \\
\hline Absorption correction & \multicolumn{2}{|c|}{ Semi-empirical from equivalents } \\
\hline Max. and min. transmission & \multicolumn{2}{|l|}{0.7532 and 0.5979} \\
\hline Refinement method & \multicolumn{2}{|c|}{ Full-matrix least-squares on $\mathrm{F}^{2}$} \\
\hline Data / restraints / parameters & \multicolumn{2}{|l|}{$5399 / 0 / 388$} \\
\hline Goodness-of-fit on $\mathrm{F}^{2}$ & \multicolumn{2}{|l|}{1.026} \\
\hline Final $\mathrm{R}$ indices [I>2sigma(I)] & \multicolumn{2}{|c|}{$\mathrm{R} 1=0.0406, \mathrm{wR} 2=0.1100$} \\
\hline $\mathrm{R}$ indices (all data) & \multicolumn{2}{|c|}{$\mathrm{R} 1=0.0429, \mathrm{wR} 2=0.1121$} \\
\hline Absolute structure parameter & \multicolumn{2}{|l|}{$0.05(8)$} \\
\hline Extinction coefficient & \multicolumn{2}{|l|}{$\mathrm{n} / \mathrm{a}$} \\
\hline Largest diff. peak and hole & \multicolumn{2}{|c|}{0.148 and -0.209 e. $\AA^{-3}$} \\
\hline
\end{tabular}


Table S2. Comparison of experimental ${ }^{13} \mathrm{C}$ NMR data with calculated ones of compounds 8, 9, 11 and 18

\begin{tabular}{|c|c|c|c|c|c|c|c|c|c|}
\hline No. & $\begin{array}{c}\text { Exp. } \\
8\end{array}$ & $\begin{array}{c}\text { Calcd. } \\
6 R-8\end{array}$ & $\begin{array}{c}\text { Calcd. } \\
6 S-8\end{array}$ & $\begin{array}{c}\text { Exp. } \\
9\end{array}$ & $\begin{array}{c}\text { Calcd. } \\
9\end{array}$ & $\begin{array}{c}\text { Exp. } \\
\mathbf{1 1}\end{array}$ & $\begin{array}{c}\text { Calcd. } \\
\mathbf{1 1}\end{array}$ & $\begin{array}{c}\text { Exp. } \\
\mathbf{1 8}\end{array}$ & $\begin{array}{c}\text { Calcd. } \\
18\end{array}$ \\
\hline 1 & 75.9 & 75.6 & 74.8 & 77.5 & 77.9 & 78.3 & 76.5 & 86.6 & 86.7 \\
\hline 2 & 66.1 & 67.4 & 68.0 & 27.6 & 29.3 & 71.3 & 72.6 & 24.6 & 27.6 \\
\hline 3 & 77 & 76.8 & 78.4 & 76.7 & 77.0 & 72.9 & 74.7 & 77.2 & 78.6 \\
\hline 4 & 38.8 & 40.2 & 42.1 & 37.1 & 38.9 & 40.7 & 44.1 & 37.4 & 38.8 \\
\hline 5 & 42.9 & 43.6 & 45.9 & 45.7 & 45.0 & 51.0 & 52.8 & 45.9 & 46.9 \\
\hline 6 & 71 & 72.2 & 72.2 & 71.2 & 72.1 & 70.0 & 69.2 & 70.2 & 70.0 \\
\hline 7 & 174.5 & 177.0 & 177.7 & 174.9 & 174.1 & 175.3 & 176.0 & 175.2 & 176.7 \\
\hline 8 & 147.3 & 151.7 & 154.2 & 44.0 & 45.4 & 44.8 & 48.4 & 101.5 & 102.2 \\
\hline 9 & 77.7 & 79.0 & 82.0 & 210.2 & 210.2 & 211.6 & 210.8 & 159.2 & 159.9 \\
\hline 10 & 52.3 & 53.2 & 56.9 & 45.9 & 46.7 & 45.6 & 46.7 & 48.7 & 49.7 \\
\hline 11 & 35 & 36.8 & 37.4 & 65.4 & 66.5 & 58.5 & 60.0 & 56.6 & 58.0 \\
\hline 12 & 30 & 32.6 & 32.2 & 70.4 & 72.1 & 29.4 & 30.8 & 71.5 & 73.1 \\
\hline 13 & 41.3 & 42.2 & 42.4 & 45.5 & 46.9 & 40.5 & 44.6 & 44.8 & 46.4 \\
\hline 14 & 81.6 & 84.8 & 85.0 & 79.4 & 82.1 & 80.2 & 83.2 & 160.4 & 160.3 \\
\hline 15 & 33.8 & 36.7 & 36.3 & 39.6 & 41.2 & 38.8 & 40.1 & 106.4 & 104.8 \\
\hline 16 & 170.3 & 168.1 & 168.1 & 168.4 & 166.9 & 168.8 & 165.4 & 165.1 & 160.6 \\
\hline 17 & 80.1 & 82.0 & 82.0 & 77.8 & 79.2 & 79.2 & 80.0 & 77.4 & 78.7 \\
\hline 18 & 13.6 & 15.7 & 15.2 & 15.7 & 17.2 & 21.6 & 21.6 & 13.7 & 15.2 \\
\hline 19 & 17.2 & 18.7 & 18.1 & 19.0 & 18.3 & 19.2 & 18.8 & 21.0 & 22.7 \\
\hline 20 & 121.2 & 123.8 & 123.8 & 120.4 & 122.4 & 120.9 & 123.2 & 120.8 & 122.8 \\
\hline 21 & 140 & 139.5 & 139.6 & 142.0 & 140.9 & 141.7 & 140.0 & 143.9 & 142.0 \\
\hline 22 & 109.5 & 109.8 & 109.8 & 110.0 & 110.9 & 110.3 & 110.4 & 111.3 & 113.8 \\
\hline 23 & 143 & 141.8 & 141.8 & 143.4 & 141.1 & 143.0 & 141.1 & 142.4 & 139.3 \\
\hline 28 & 22.4 & 22.8 & 25.6 & 22.8 & 22.8 & 15.6 & 16.2 & 22.6 & 24.7 \\
\hline 29 & 27.7 & 28.4 & 26.7 & 28.5 & 28.7 & 29.0 & 27.6 & 26.5 & 27.2 \\
\hline 30 & 110.7 & 112.1 & 110.0 & 10.2 & 11.3 & 10.2 & 11.6 & 10.3 & 11.5 \\
\hline
\end{tabular}


Table S3. Re-optimized conformers, energies and proportions for $\mathbf{6} \boldsymbol{R}-\mathbf{8}$

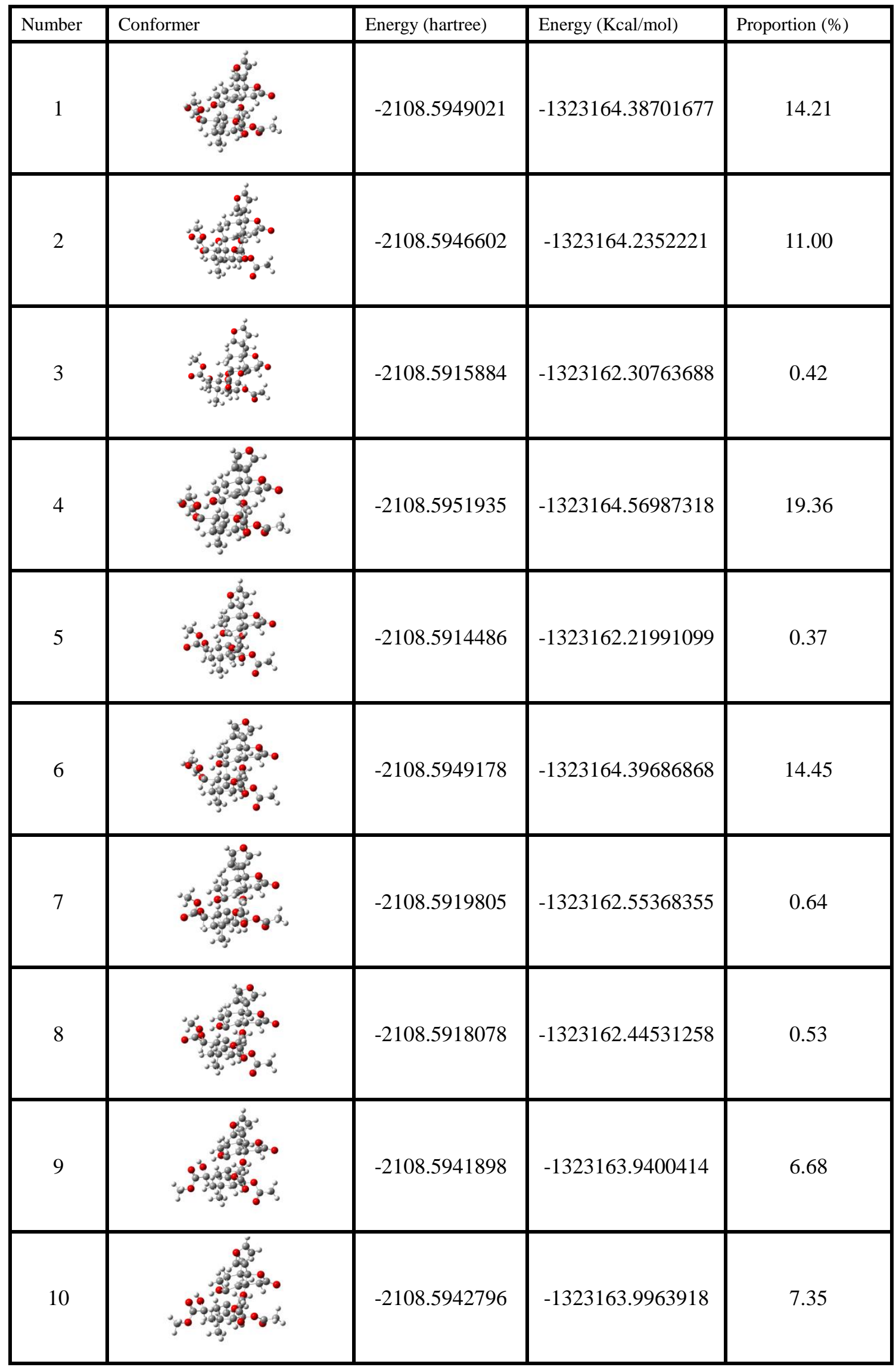




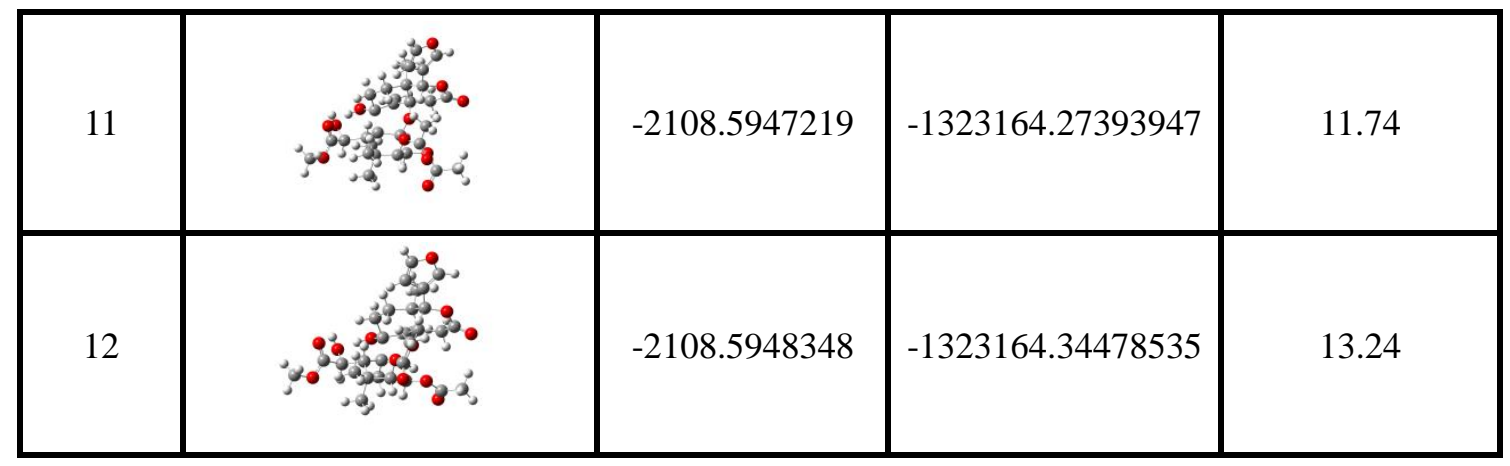


Table S4. Re-optimized conformers, energies and proportions for $\mathbf{6 S - 8}$

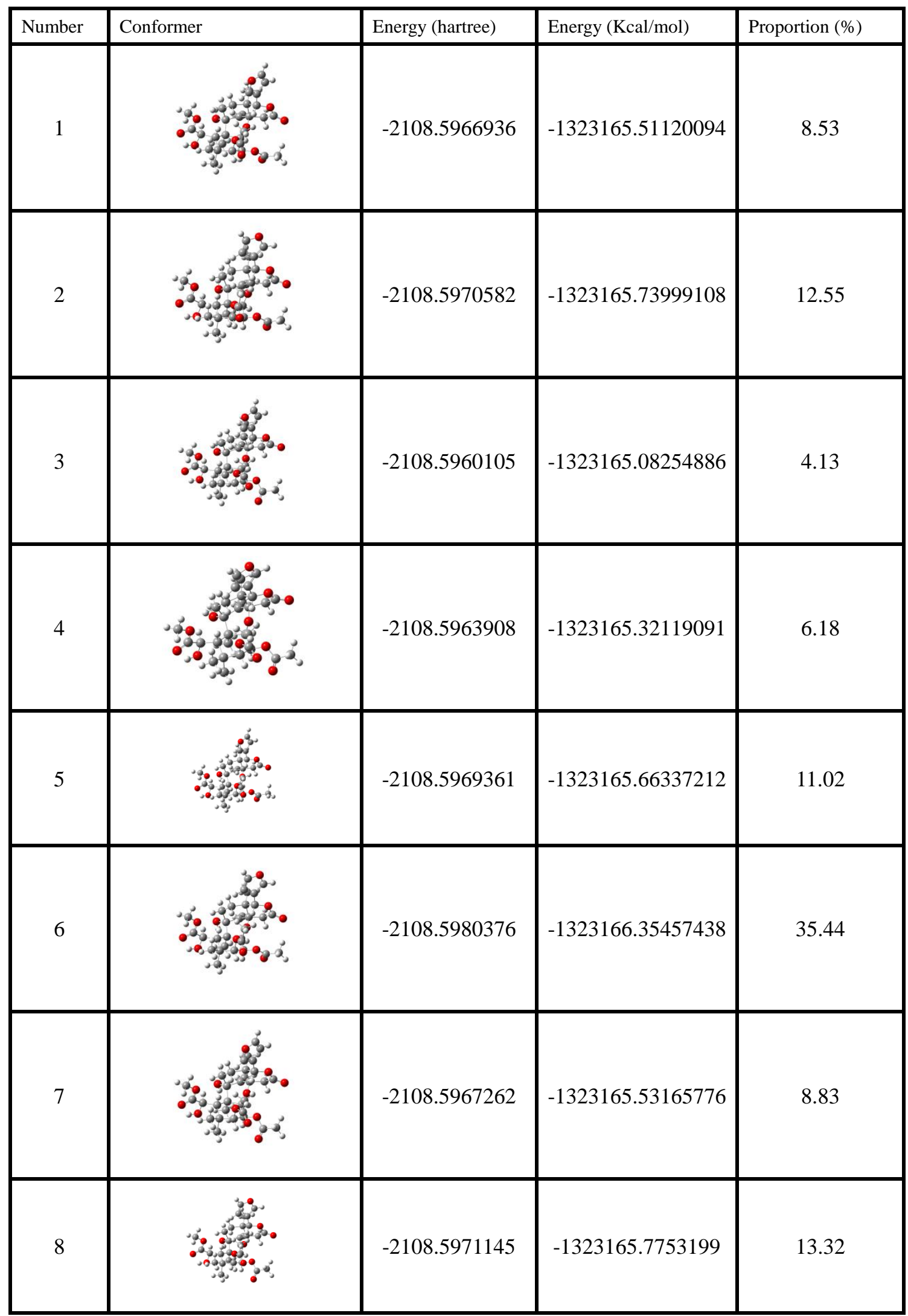


Table S5. Re-optimized conformers, energies and proportions for 9

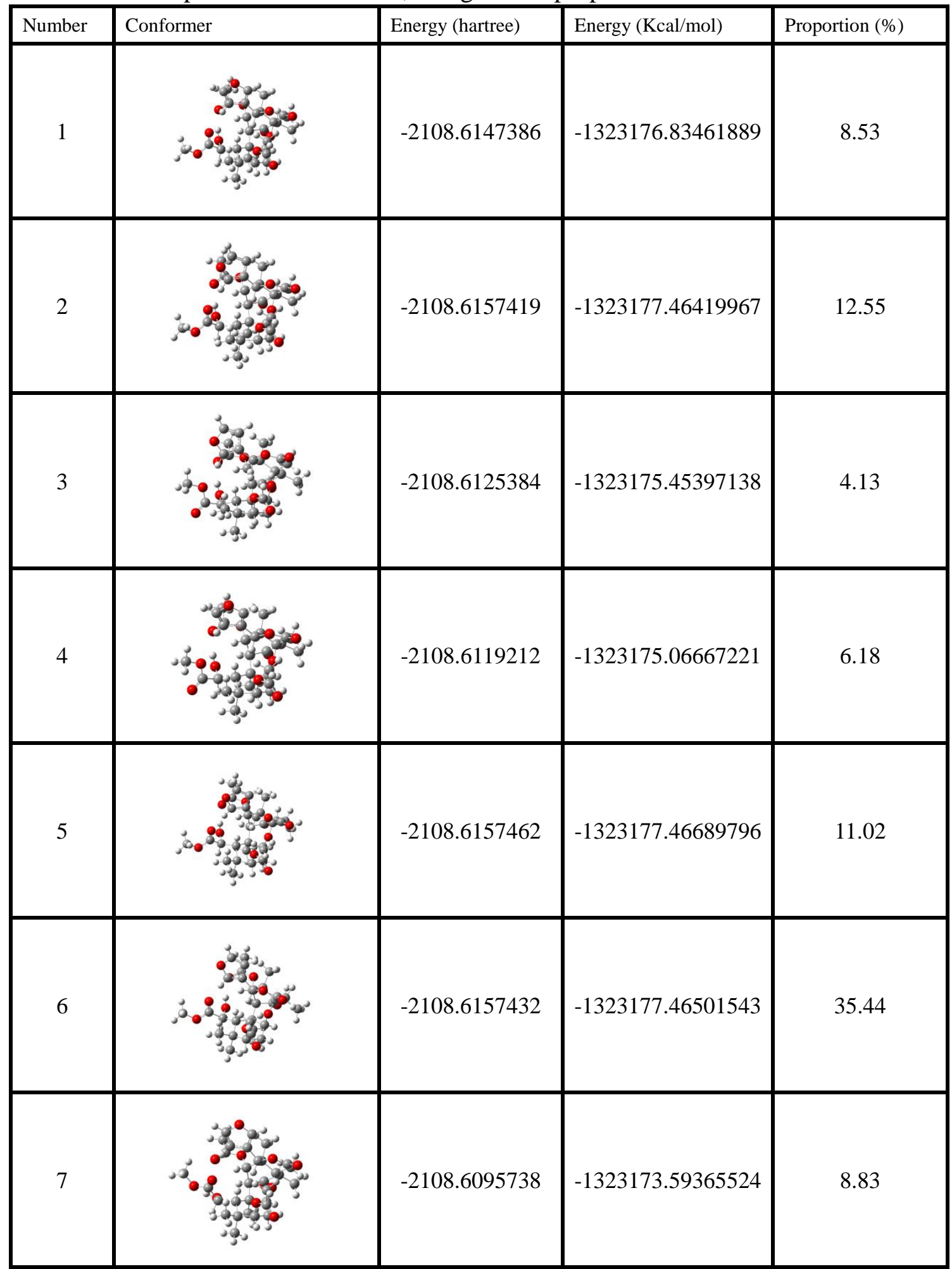


Table S6. Re-optimized conformers, energies and proportions for $\mathbf{1 1}$

\begin{tabular}{|c|c|c|c|c|}
\hline Number & Conformer & Energy (hartree) & Energy $(\mathrm{Kcal} / \mathrm{mol})$ & Proportion (\%) \\
\hline 1 & & -2034.5604732 & -1276707.04253773 & 31.02 \\
\hline 2 & & -2034.5599825 & -1276706.73461858 & 18.43 \\
\hline 3 & & -2034.5600960 & -1276706.80584096 & 20.79 \\
\hline 4 & & -2034.5595881 & -1276706.48712863 & 12.13 \\
\hline 5 & & -2034.5594598 & -1276706.4066191 & 10.59 \\
\hline 6 & & -2034.5589684 & -1276706.09826068 & 6.29 \\
\hline 7 & & -2034.5569587 & -1276704.83715384 & 0.75 \\
\hline
\end{tabular}


Table S7. Re-optimized conformers, energies and proportions for $\mathbf{1 5}$

\begin{tabular}{|c|c|c|c|c|}
\hline Number & Conformer & Energy (hartree) & Energy (Kcal/mol) & Proportion (\%) \\
\hline 1 & & -1879.4830981 & -1179394.43888873 & 48.57 \\
\hline 2 & & -1879.4823054 & -1179394.43888873 & 20.96 \\
\hline 3 & & -1879.4764679 & -1179390.27837193 & 0.04 \\
\hline 4 & & -1879.4767826 & -1179390.47584933 & 0.06 \\
\hline 5 & & -1879.4826550 & -1179394.16083905 & 30.36 \\
\hline
\end{tabular}


Table S8. Re-optimized conformers, energies and proportions for $\mathbf{1 8}$

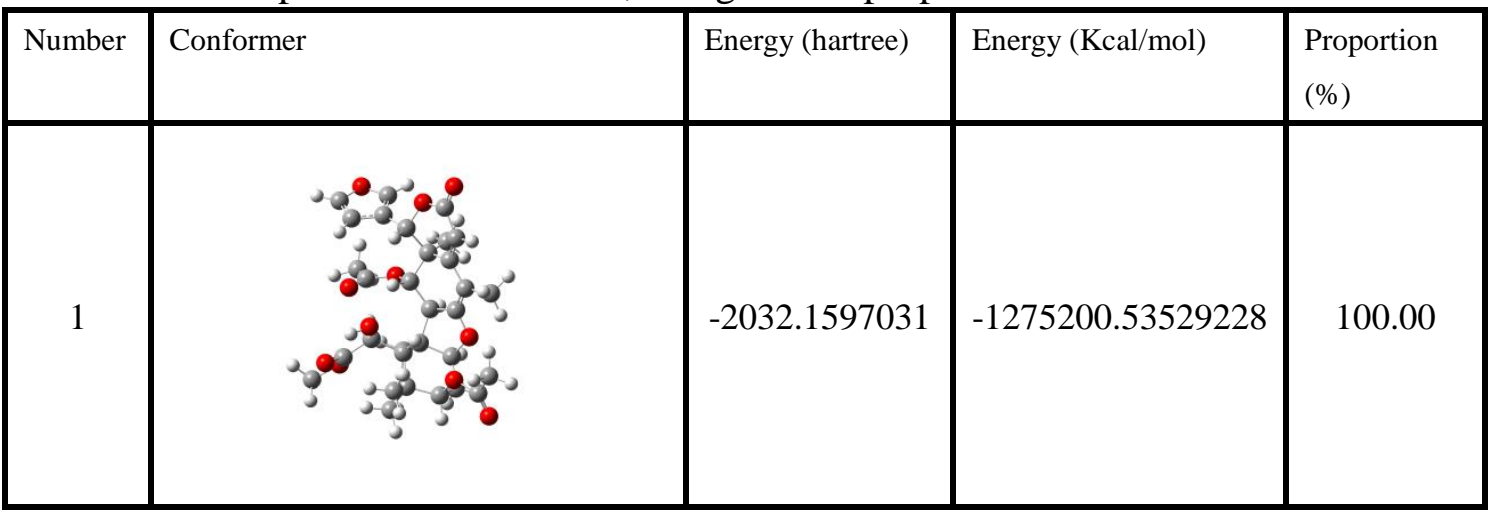


Table S9. Antimalarial activity of compounds 1, 5-7, 11 and 12

\begin{tabular}{llllllll}
\hline \hline compds & $\mathbf{1}$ & $\mathbf{5}$ & $\mathbf{6}$ & $\mathbf{7}$ & $\mathbf{1 1}$ & $\mathbf{1 2}$ & Artemisinin \\
\hline $\mathrm{IC}_{50}$ & $20.0 \pm$ & $16.3 \pm$ & $12.3 \pm$ & $23.1 \pm$ & $16.9 \pm$ & $28.0 \pm$ & $0.013 \pm$ \\
& 2.9 & 1.7 & 0.7 & 6.2 & 1.6 & 4.7 & 0.0009 \\
\hline
\end{tabular}

Data are presented as means $\pm S D, n=3$. 
Figure S1. ${ }^{1} \mathrm{H}$ NMR spectrum of cibacciferin A (1) in $\mathrm{CDCl}_{3}$

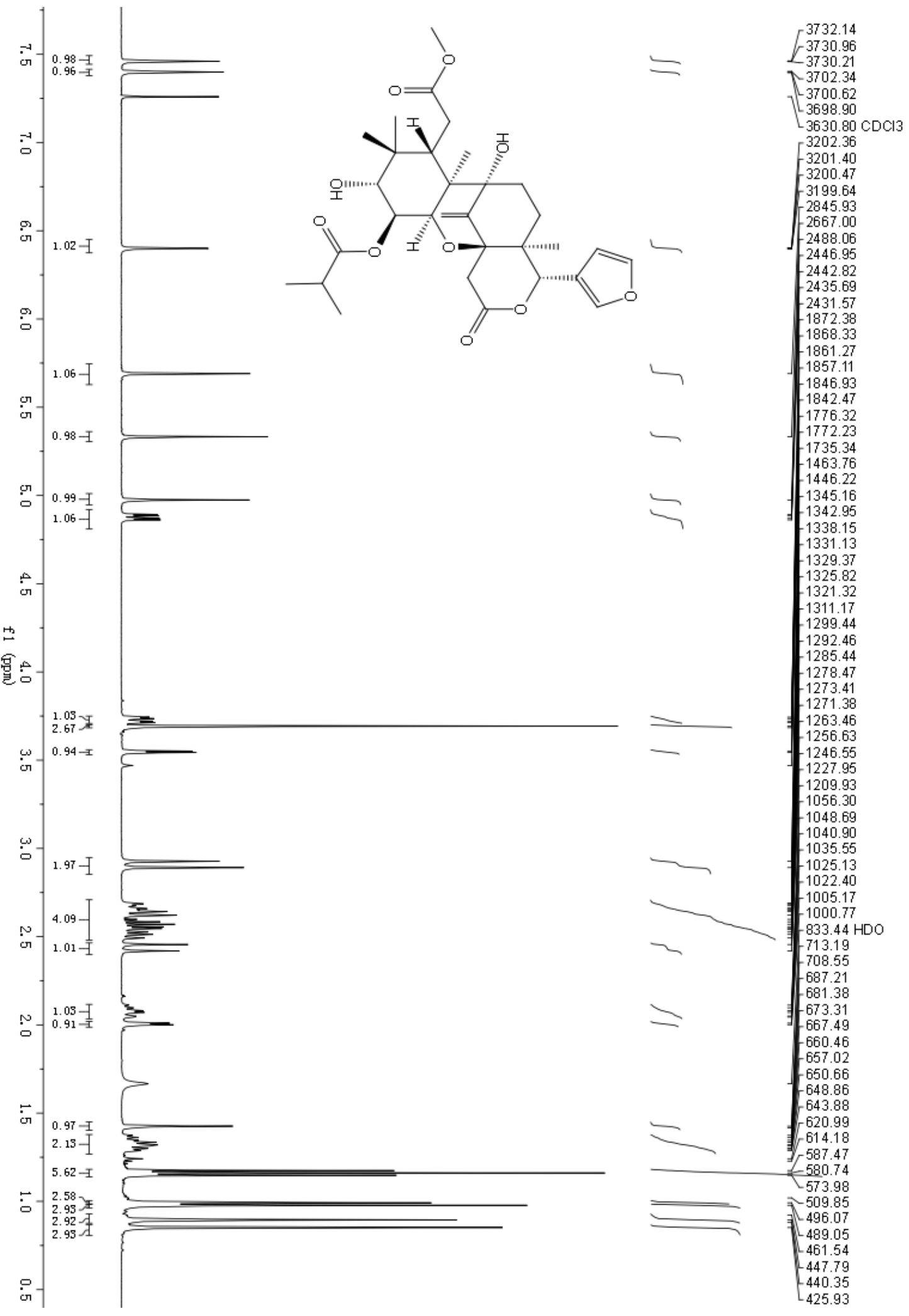


Figure S2. ${ }^{13} \mathrm{C}$ NMR spectrum of cibacciferin $\mathrm{A}(\mathbf{1})$ in $\mathrm{CDCl}_{3}$

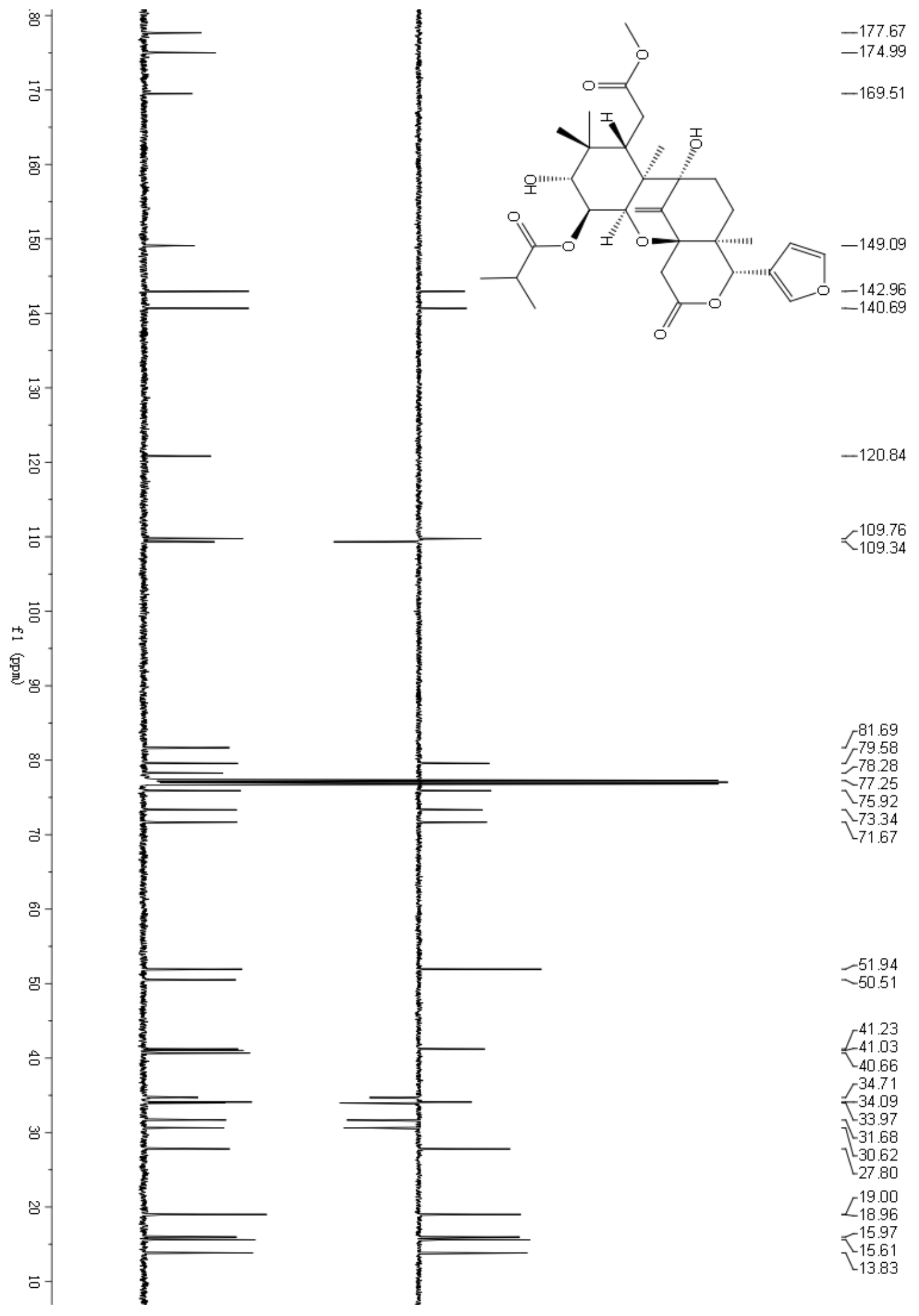


Figure S3. HSQC spectrum of cibacciferin A (1) in $\mathrm{CDCl}_{3}$

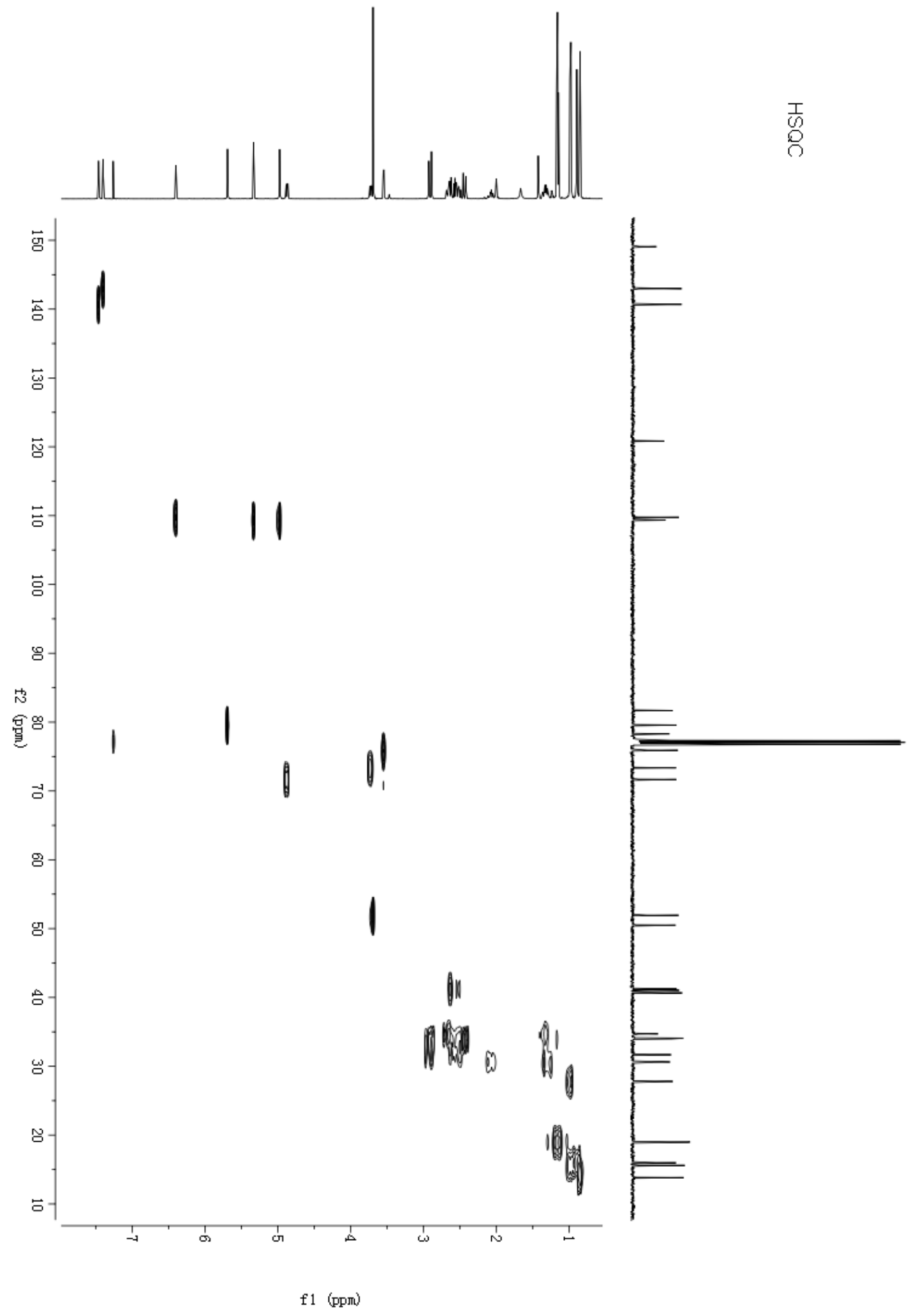


Figure S4. $\mathrm{HMBC}$ spectrum of cibacciferin $\mathrm{A}(\mathbf{1})$ in $\mathrm{CDCl}_{3}$

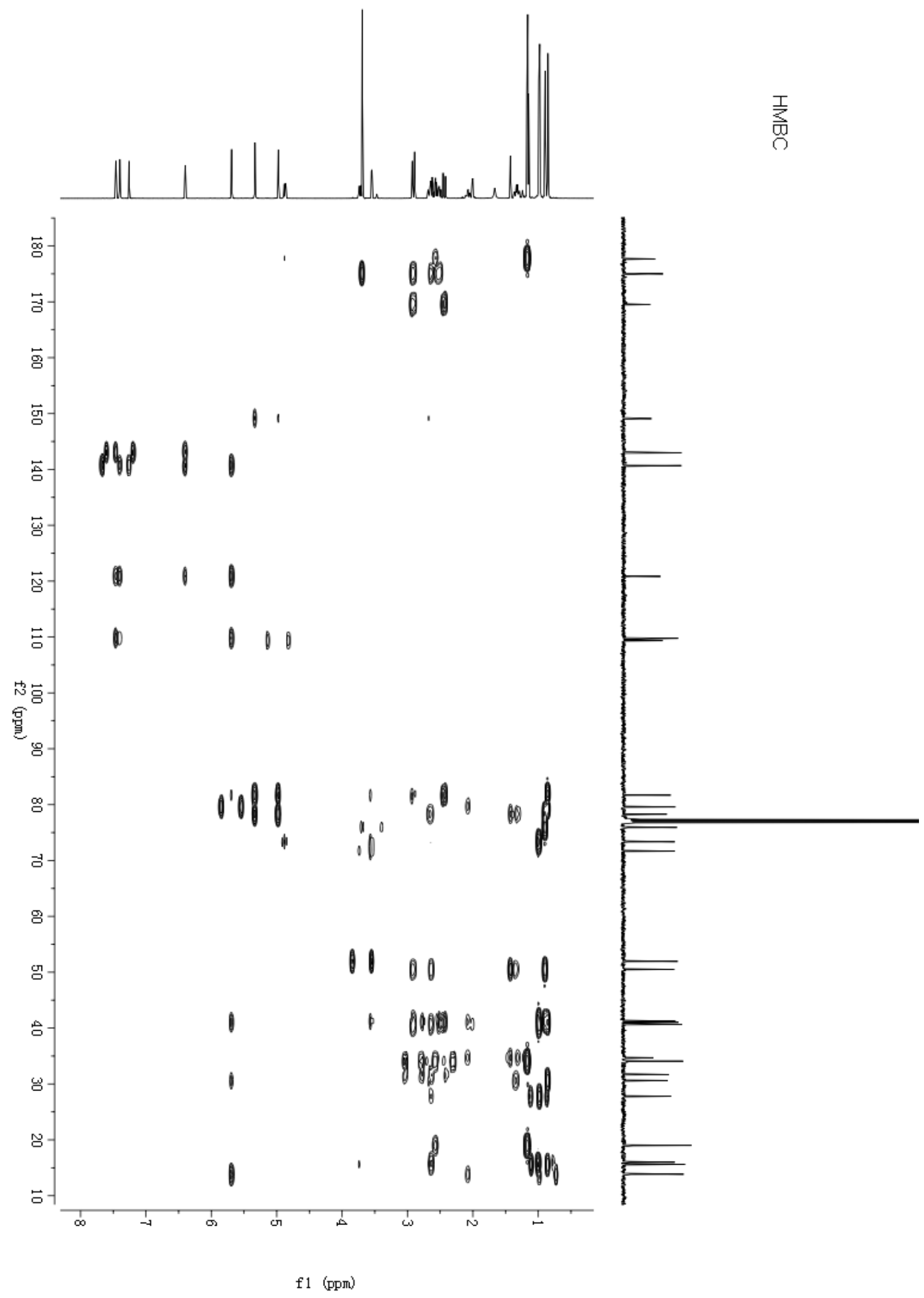


Figure S5. ROESY spectrum of cibacciferin $\mathrm{A}(\mathbf{1})$ in $\mathrm{CDCl}_{3}$

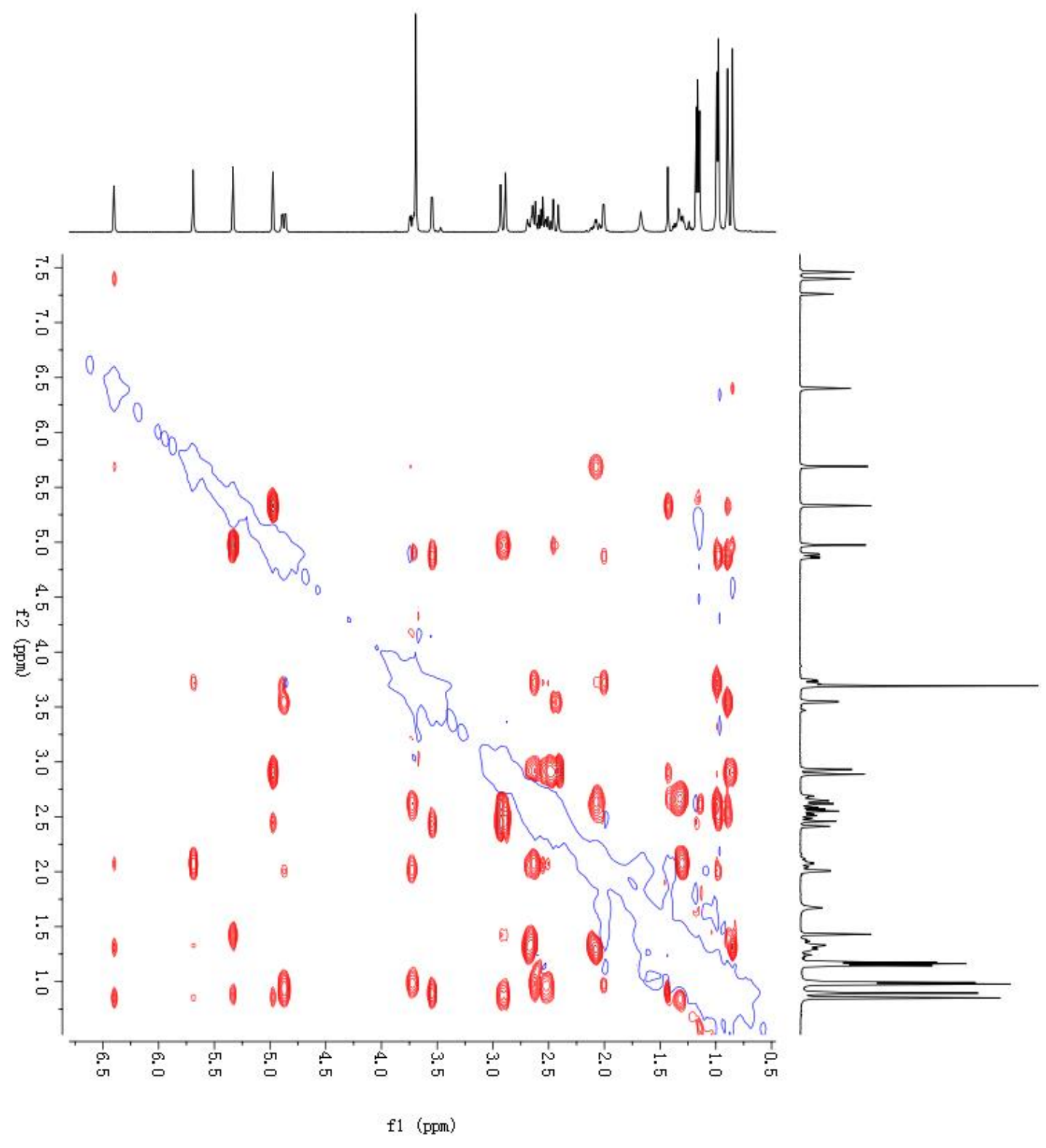


Figure S6. ESI(+)MS spectrum of cibacciferin A (1)

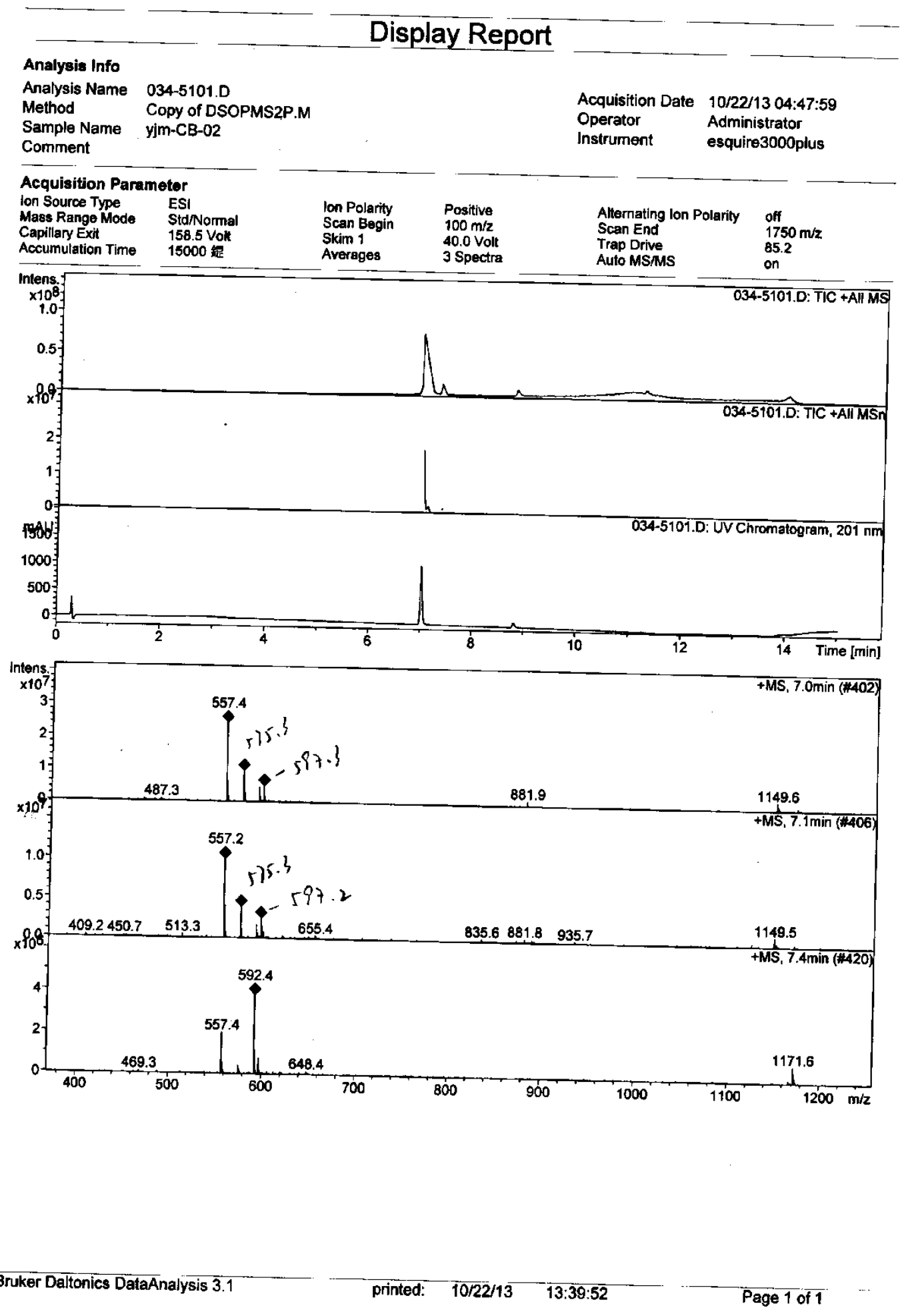


Figure S7. ESI(-)MS spectrum of cibacciferin A (1)

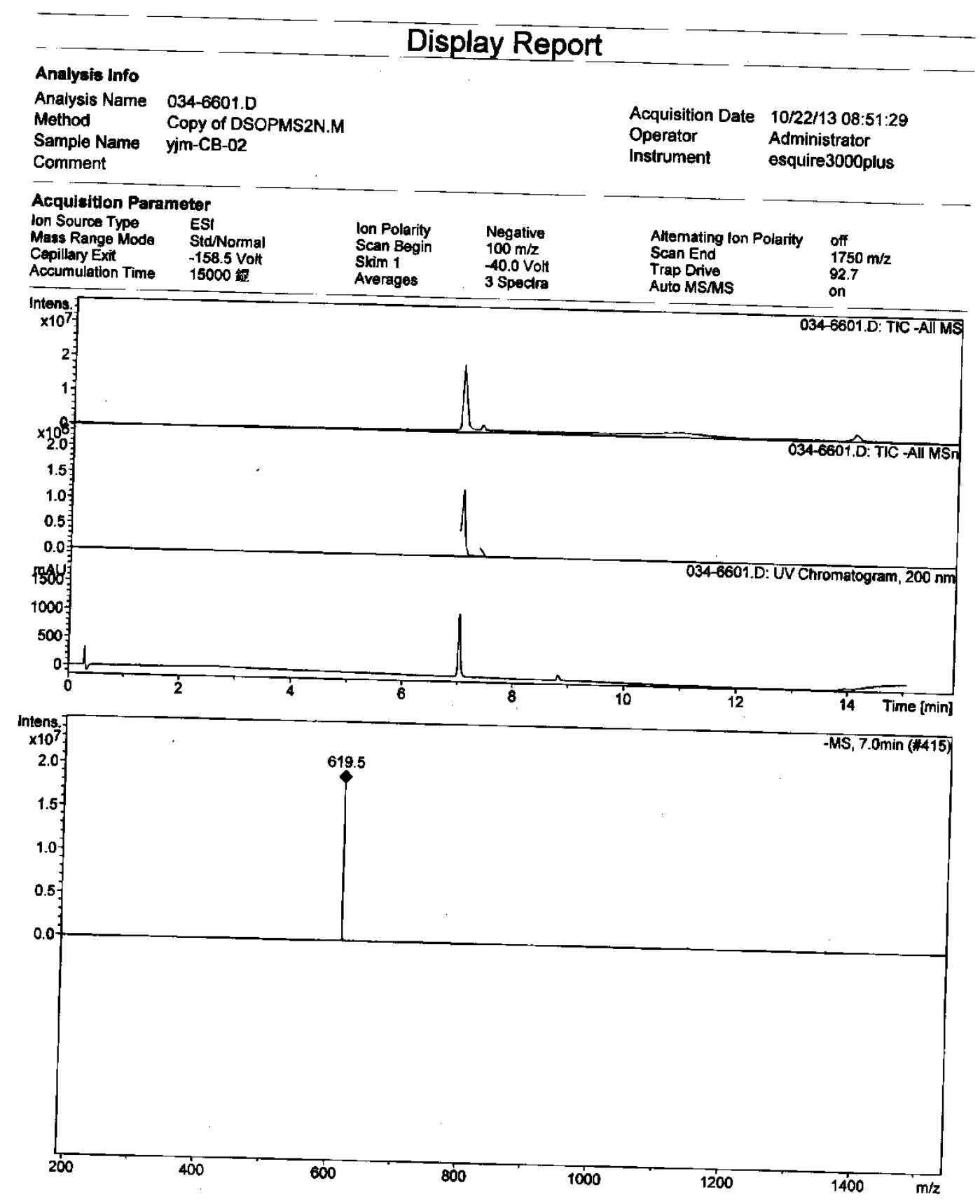


Figure S8. HRESI(+)MS spectrum of cibacciferin A (1)

Elemental Composition Report

Page 1

Single Mass Analysis

Tolerance $=3.0$ PPM $/$ DBE: $\min =-1.5, \max =50.0$

Element prediction: Off

Number of isotope peaks used for $\mathrm{i}-\mathrm{FIT}=3$

Monoisotopic Mass, Even Electron lons

242 formula(e) evaluated with 2 results within limits (up to 50 closest results for each mass)

Elements Used:

$\begin{array}{llll}\text { C: } 5-80 & \text { H: } 2-120 & \text { O: } 0-20 \quad \mathrm{Na}: 0-1\end{array}$

CB-02 LCT PXE KE324
LE

31-Oct-2013

14:01:32

CB-02_1031 31 (0.654) AM2 (Ar, 10500.0,0.00,0.70); ABS; Cm (28:41)

1: TOF MS ES+

100 597.2686

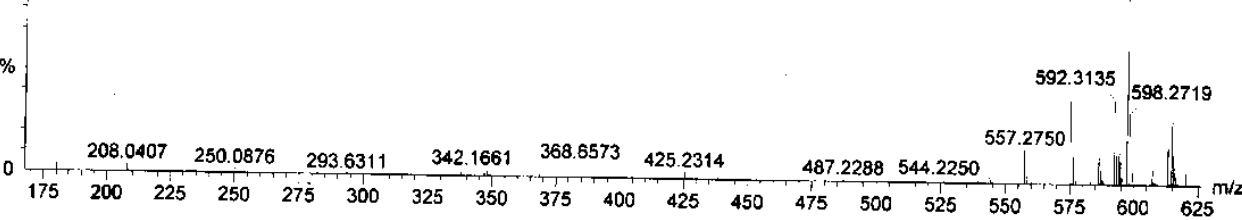

Minimum:

$\begin{array}{llll}\text { Maximum: } & 5.0 & 3.0 & -1.5 \\ & & & \end{array}$

$\begin{array}{lllllllllllll}375 & 400 & 425 & 450 & 475 & 500 & 525 & 550 & 575 & 600 & 625\end{array}$

Mass Calc. Mass mDa PEM DgE

$\begin{array}{lllll}597.2686 & 597.2676 & 2.0 & 1.7 & 10.5\end{array}$

i-FIT i-FIT (Norm) Formula

$\begin{array}{lllllllll}597.2700 & -1.4 & -2.3 & 13.5 & 70.6 & 0.2 & \mathrm{C} 31 & \mathrm{H} 42 & 010 \mathrm{Na}\end{array}$ 
Figure S9. IR spectrum of cibacciferin A (1)

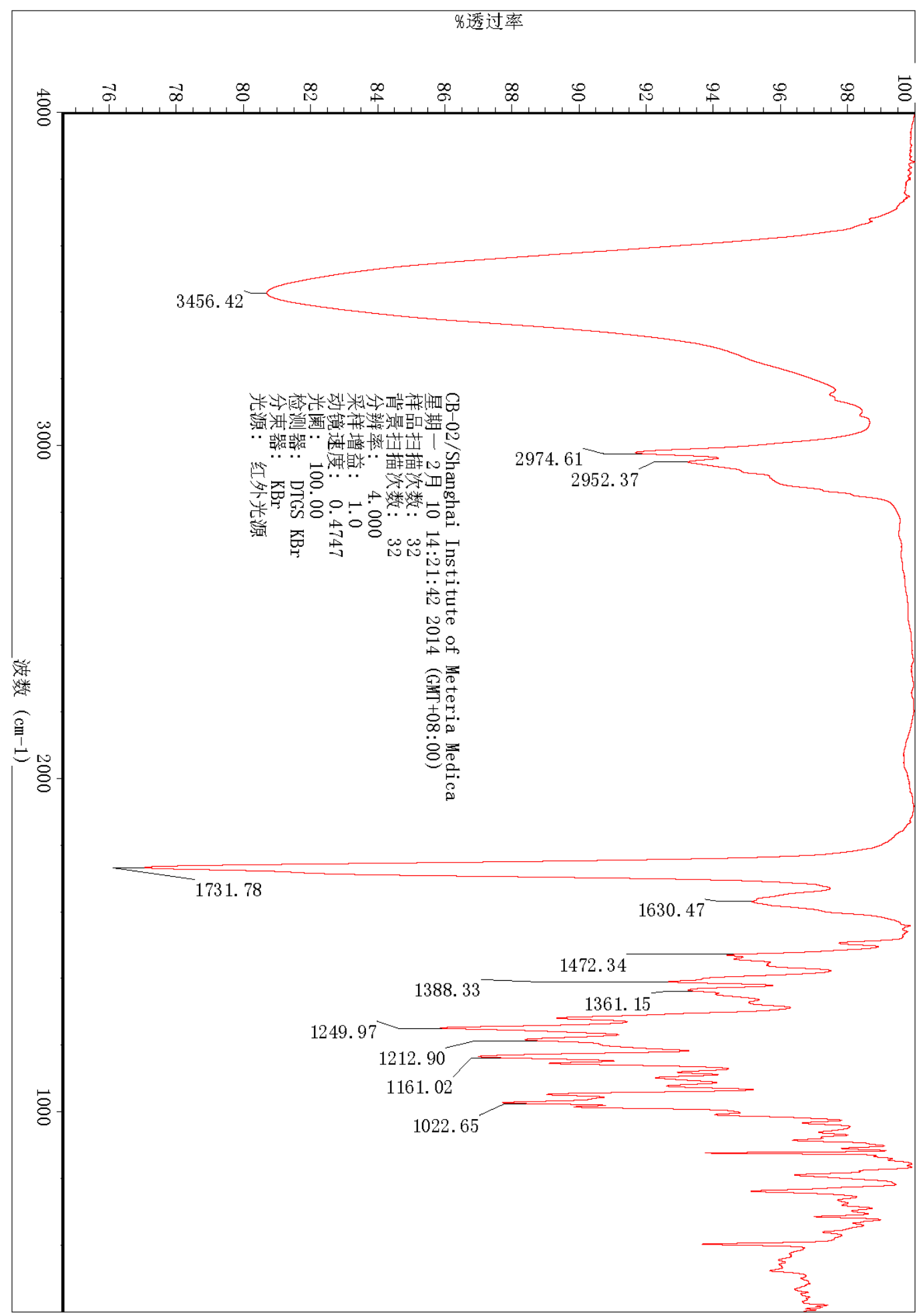


Figure S10. ${ }^{1} \mathrm{H}$ NMR spectrum of $11 \alpha$-acetoxycibacciferin $\mathrm{A}(2)$ in $\mathrm{CDCl}_{3}$

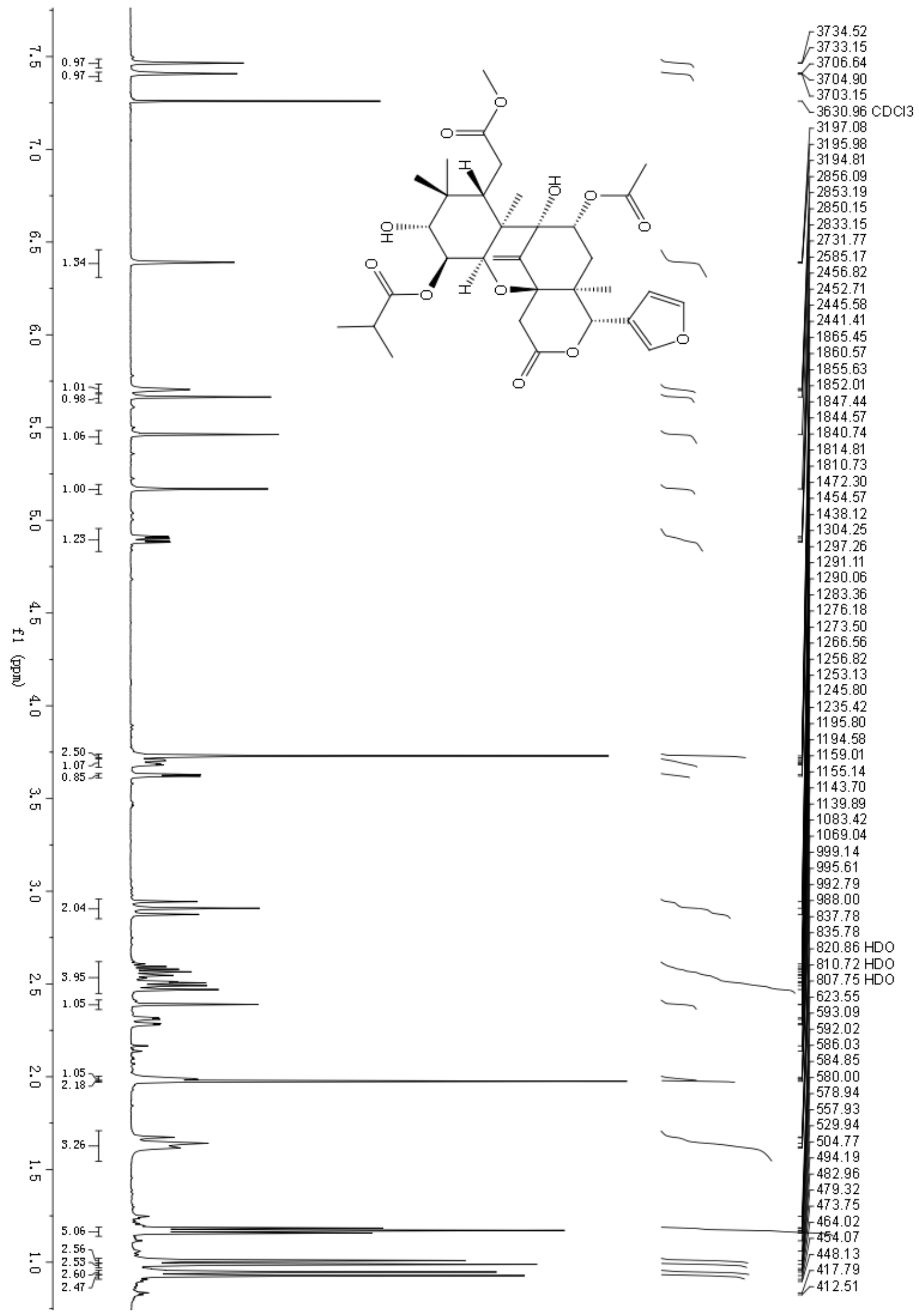


Figure S11. ${ }^{13} \mathrm{C}$ NMR spectrum of $11 \alpha$-acetoxycibacciferin $\mathrm{A}(2)$ in $\mathrm{CDCl}_{3}$

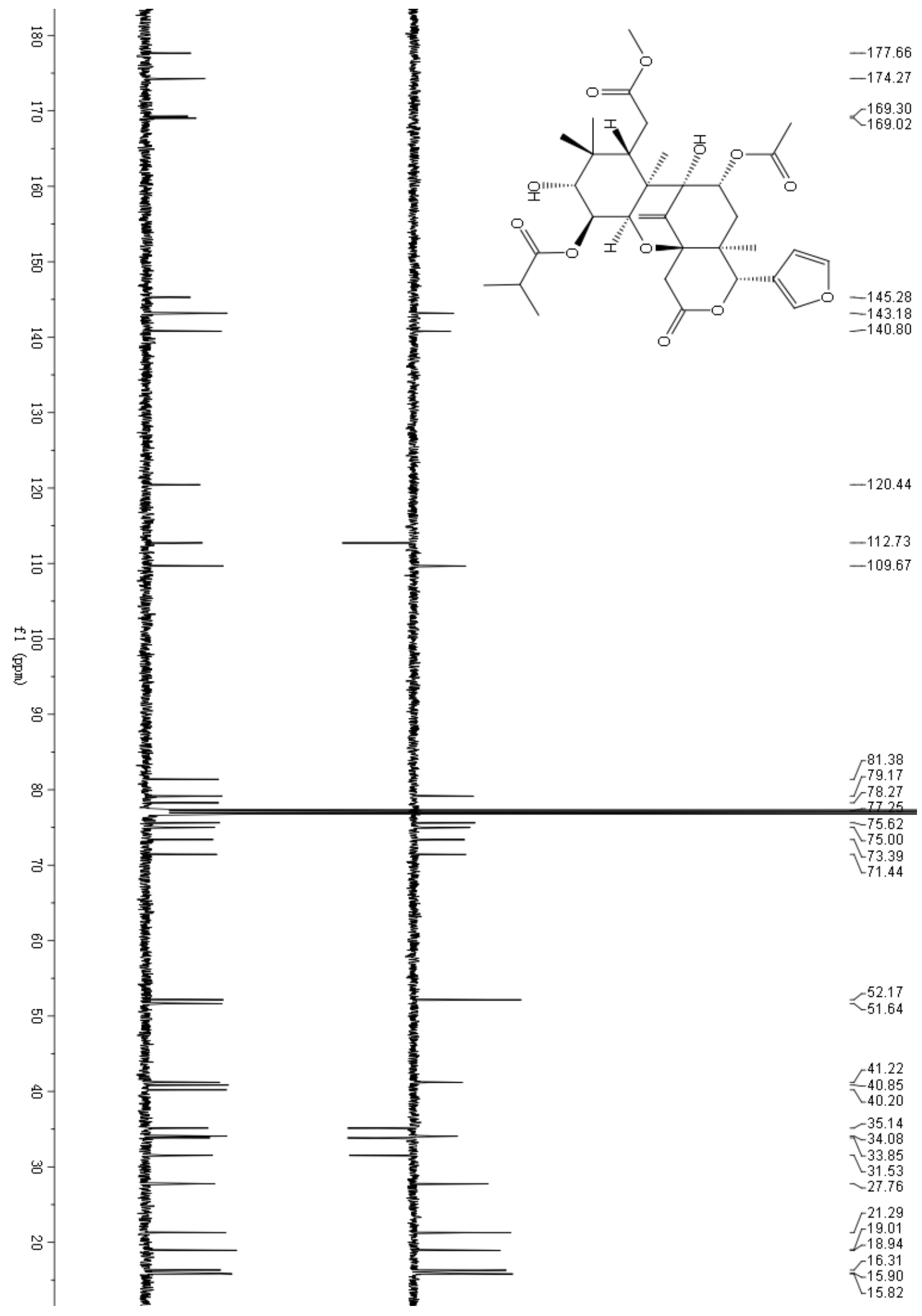


Figure S12. HSQC spectrum of $11 \alpha$-acetoxycibacciferin $\mathrm{A}(2)$ in $\mathrm{CDCl}_{3}$

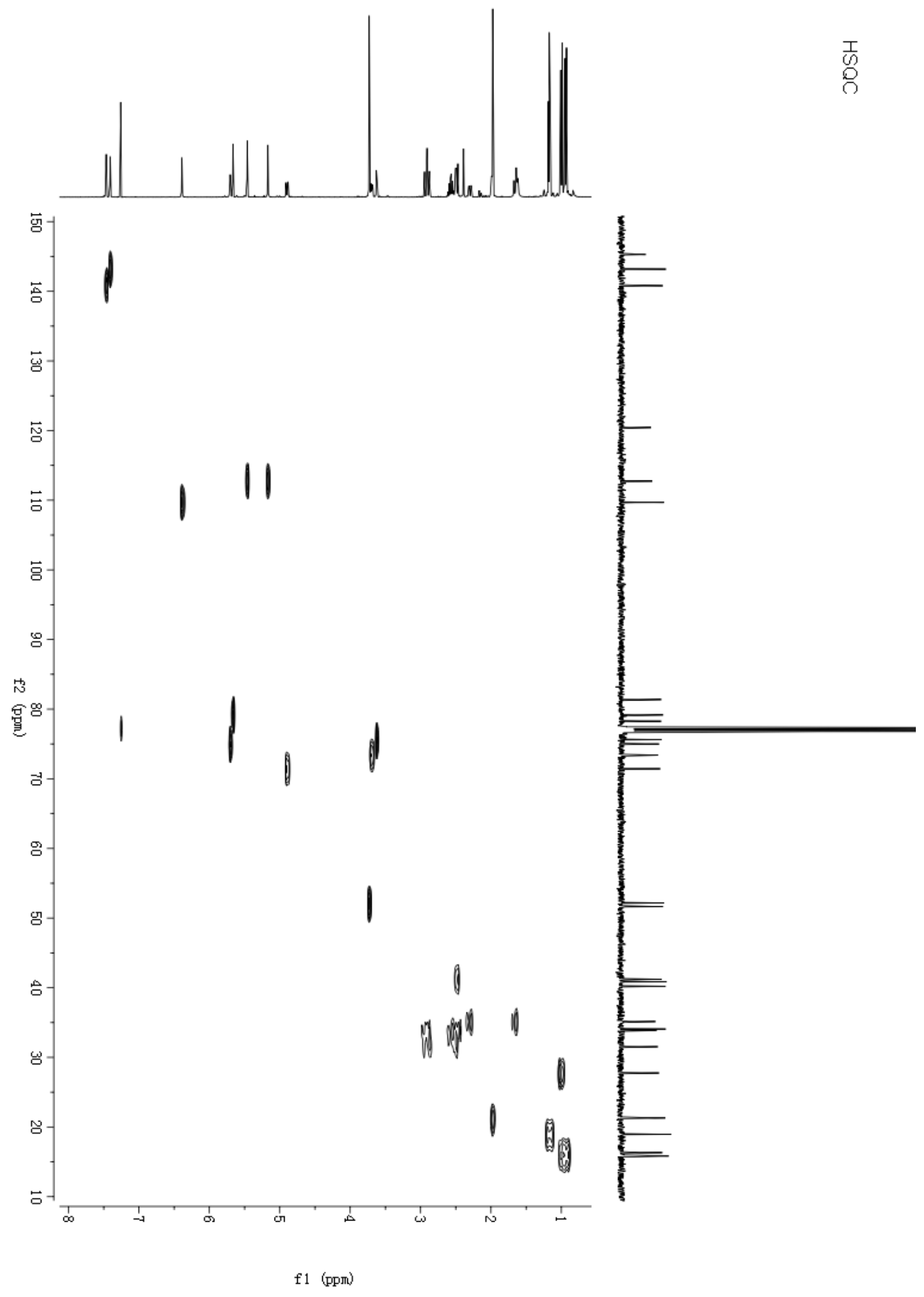


Figure S13. $\mathrm{HMBC}$ spectrum of $11 \alpha$-acetoxycibacciferin A (2) in $\mathrm{CDCl}_{3}$

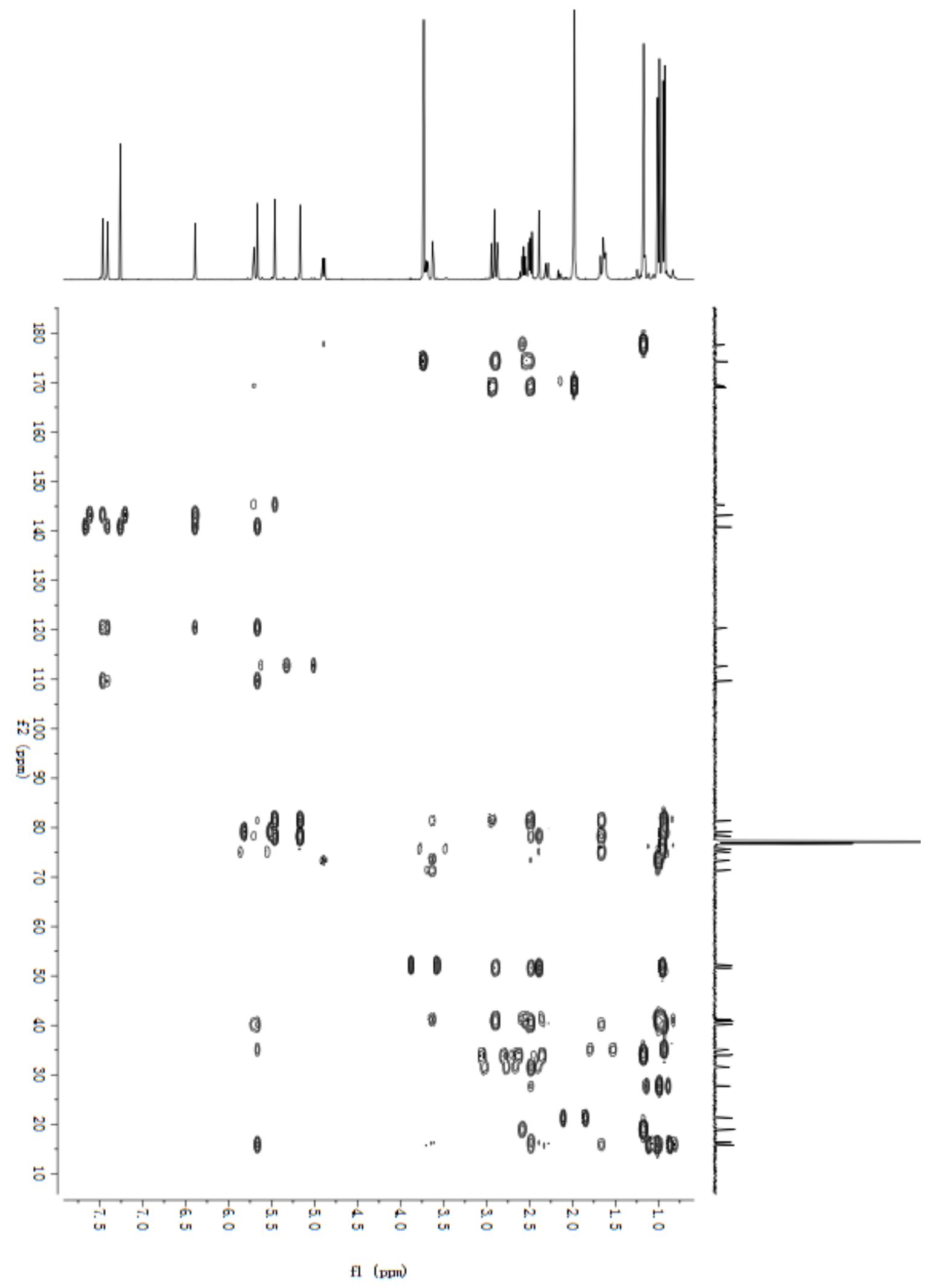


Figure S14. ESI(+)MS spectrum of $11 \alpha$-acetoxycibacciferin A (2)

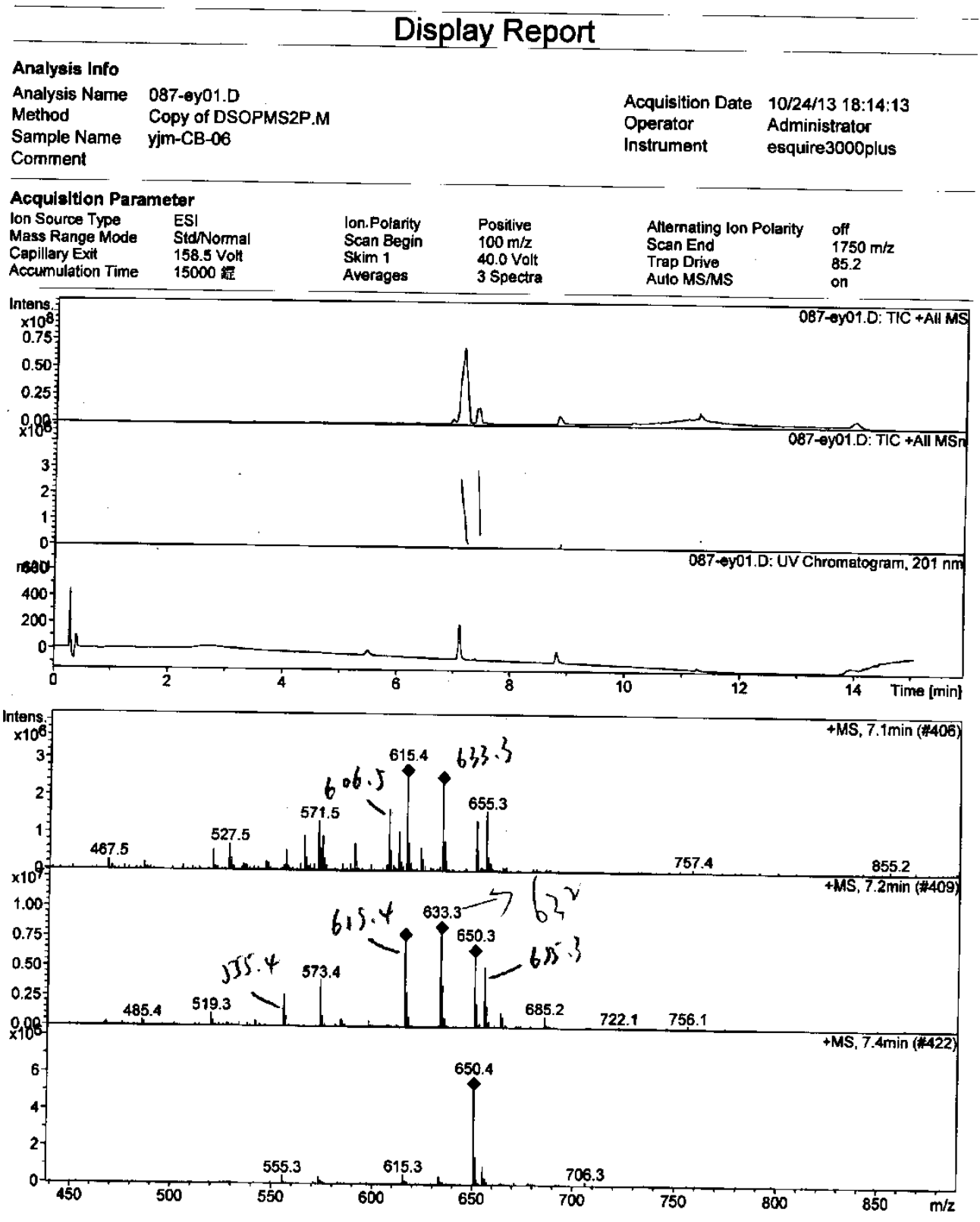


Figure S15. ESI(-)MS spectrum of $11 \alpha$-acetoxycibacciferin A (2)

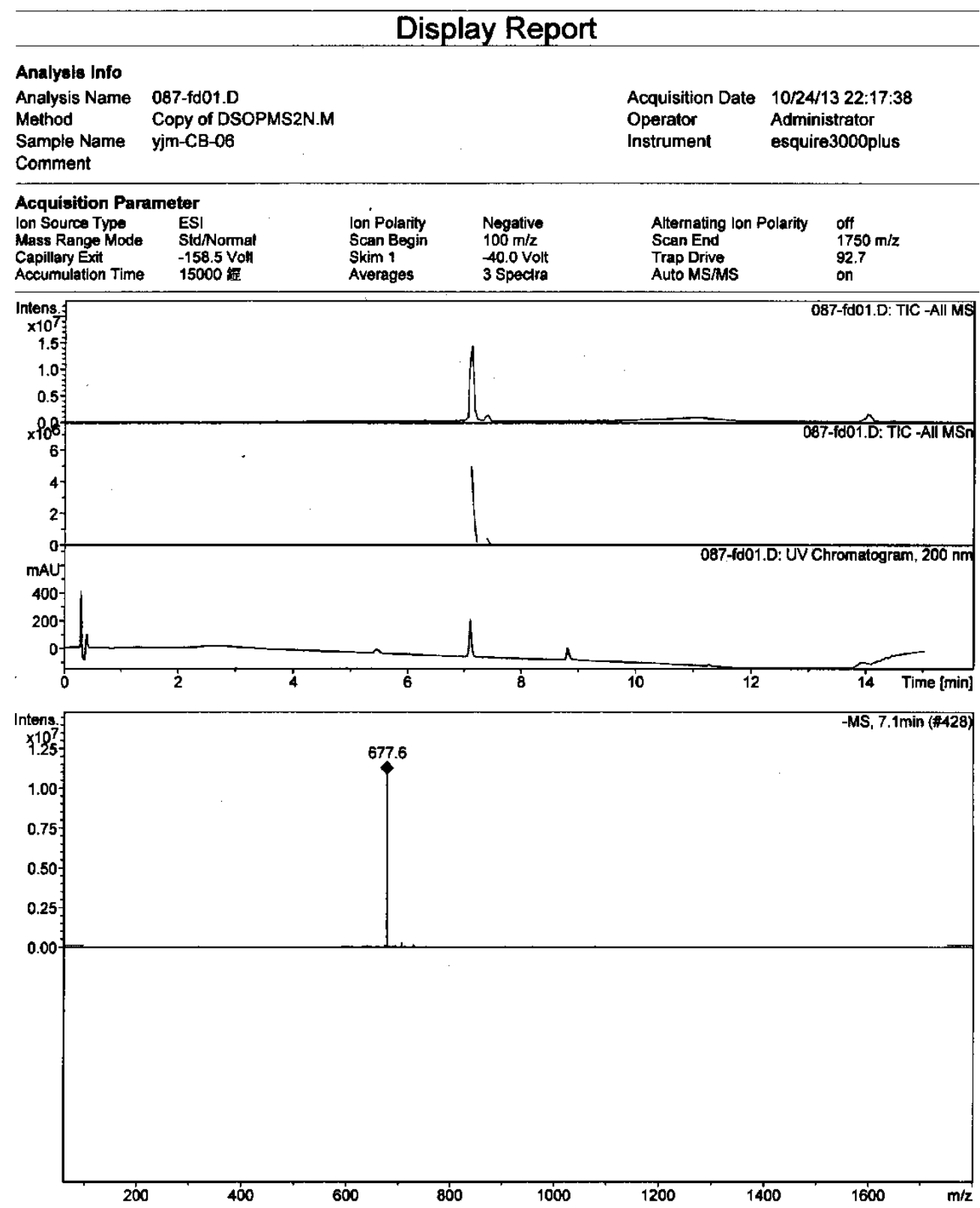


Figure S16. HRESI(+)MS spectrum of $11 \alpha$-acetoxycibacciferin A (2)

\section{Elemental Composition Report}

Single Mass Analysis

Tolerance $=3.0 \mathrm{PPM} / \mathrm{DBE}: \min =-1.5, \max =50.0$
Element prediction: Off

Number of isotope peaks used for $\mathrm{i}-\mathrm{F} I \mathrm{~T}=3$

Monoisotopic Mass, Even Electron lons

271 formula(e) evaluated with 1 results within limits (up to 50 closest results for each mass)
Elements Used:

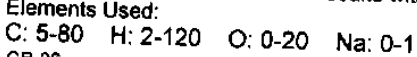

$\begin{array}{lll}\text { CB-08 } & \text { H: } 2-120 & \text { O: } 0-20 \text { Na: 0-1 }\end{array}$

CB-06_1031 23 (0.495) AM2 (Ar, 10500.0,0.00,0.70); ABS; Cm (17:28)

100

$\%$

$\%$

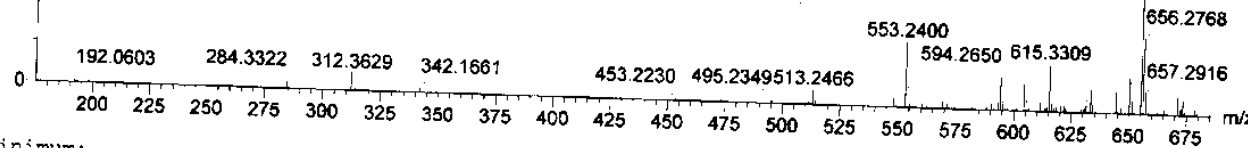

Minimum:

Maximum: $\quad \begin{array}{lll}5.0 & 3.0 & -1.5 \\ & 50.0\end{array}$

Mass Calc. Mass mDa PPM DBE

$\begin{array}{llll}655.2734 \quad 655.2730 & 0.4 & 0.6\end{array}$

$\begin{array}{llllllllllllll} & 11.5 & 86.0 & 0.0 & \text { C33 } & \text { H4 } & 012 & \text { Na }\end{array}$


Figure S17. IR spectrum of $11 \alpha$-acetoxycibacciferin A (2)

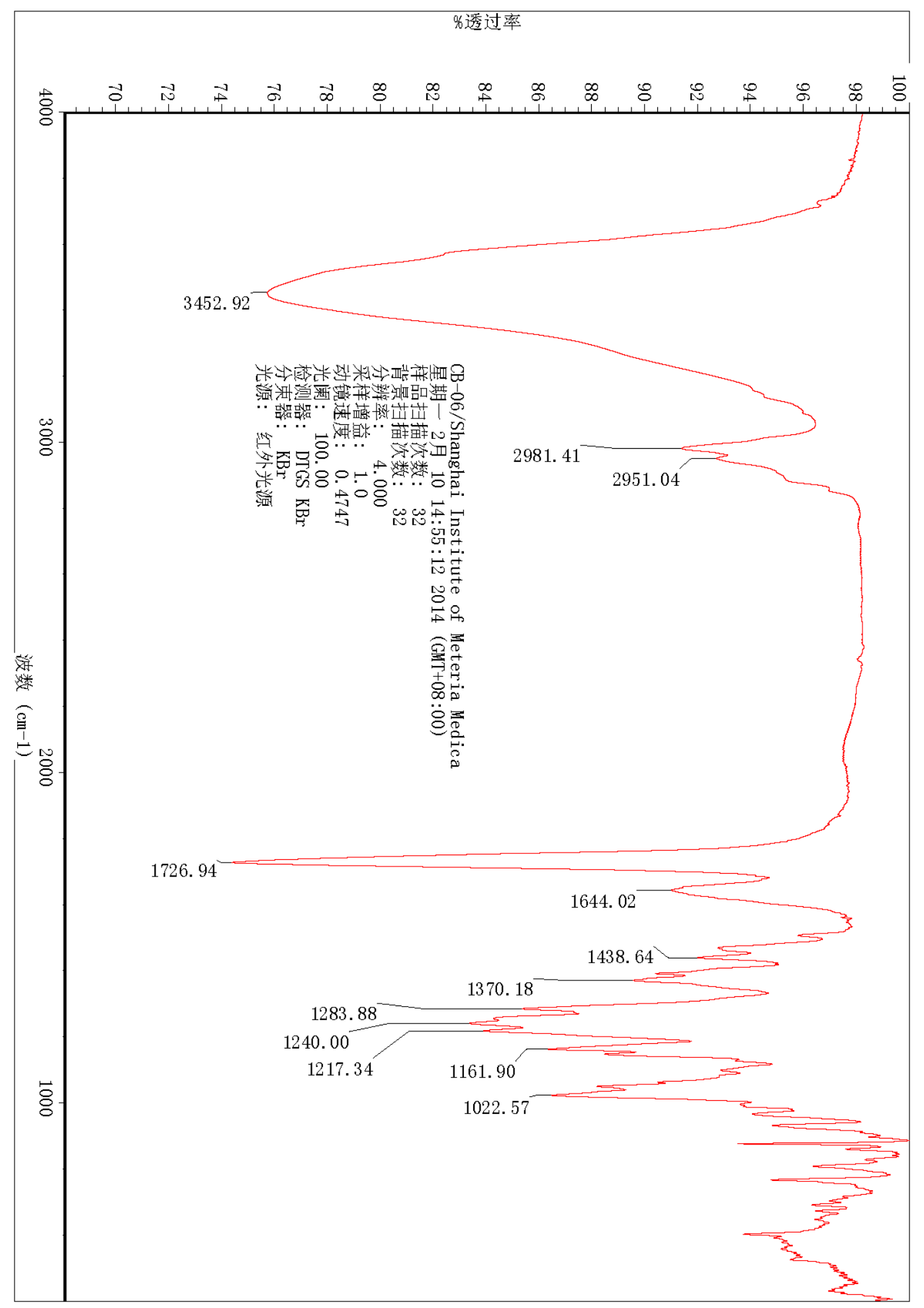


Figure S18. ${ }^{1} \mathrm{H}$ NMR spectrum of cibacciferin $\mathrm{B}(3)$ in $\mathrm{CDCl}_{3}$

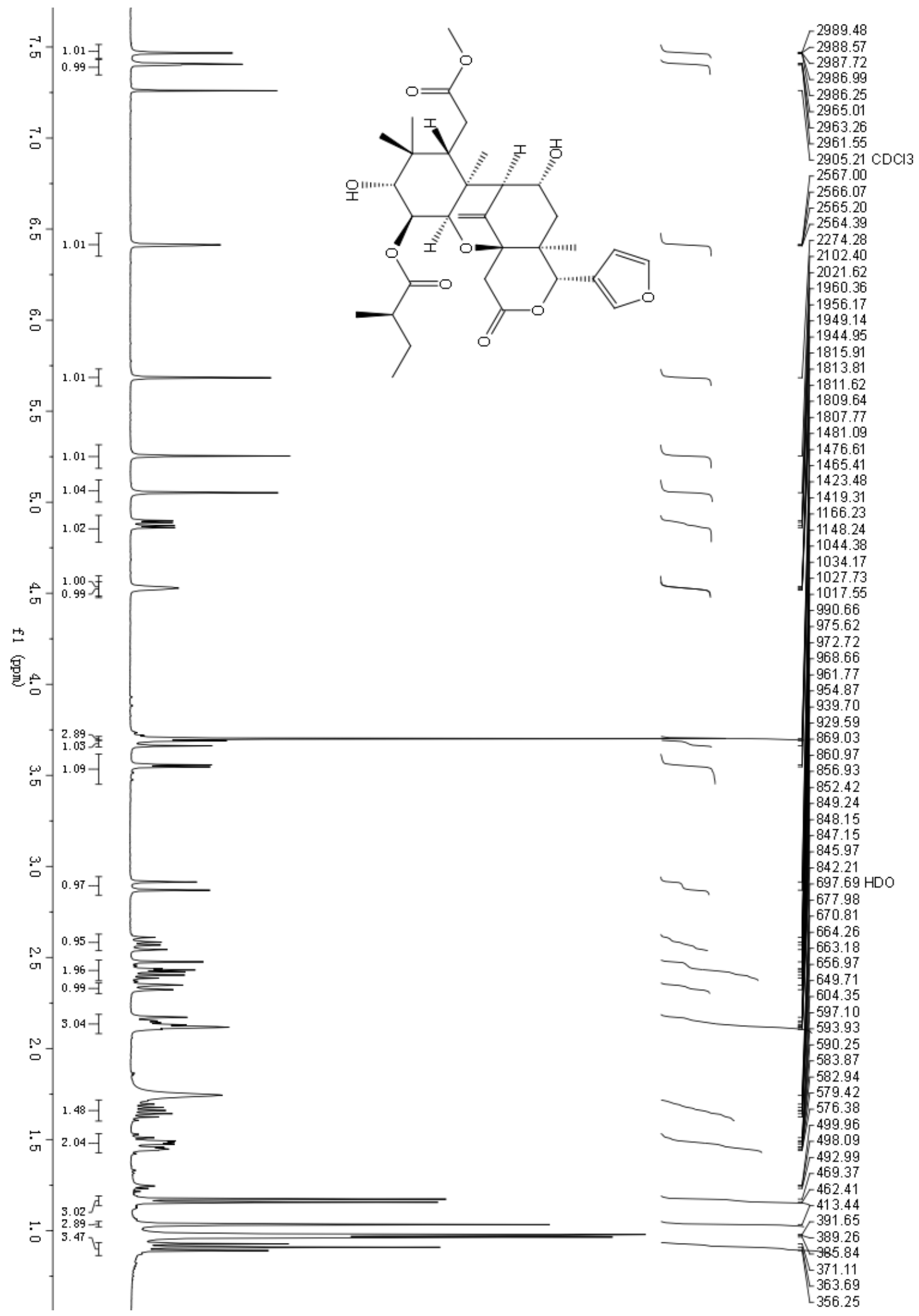


Figure S19. ${ }^{13} \mathrm{C}$ NMR spectrum of cibacciferin $\mathrm{B}(3)$ in $\mathrm{CDCl}_{3}$

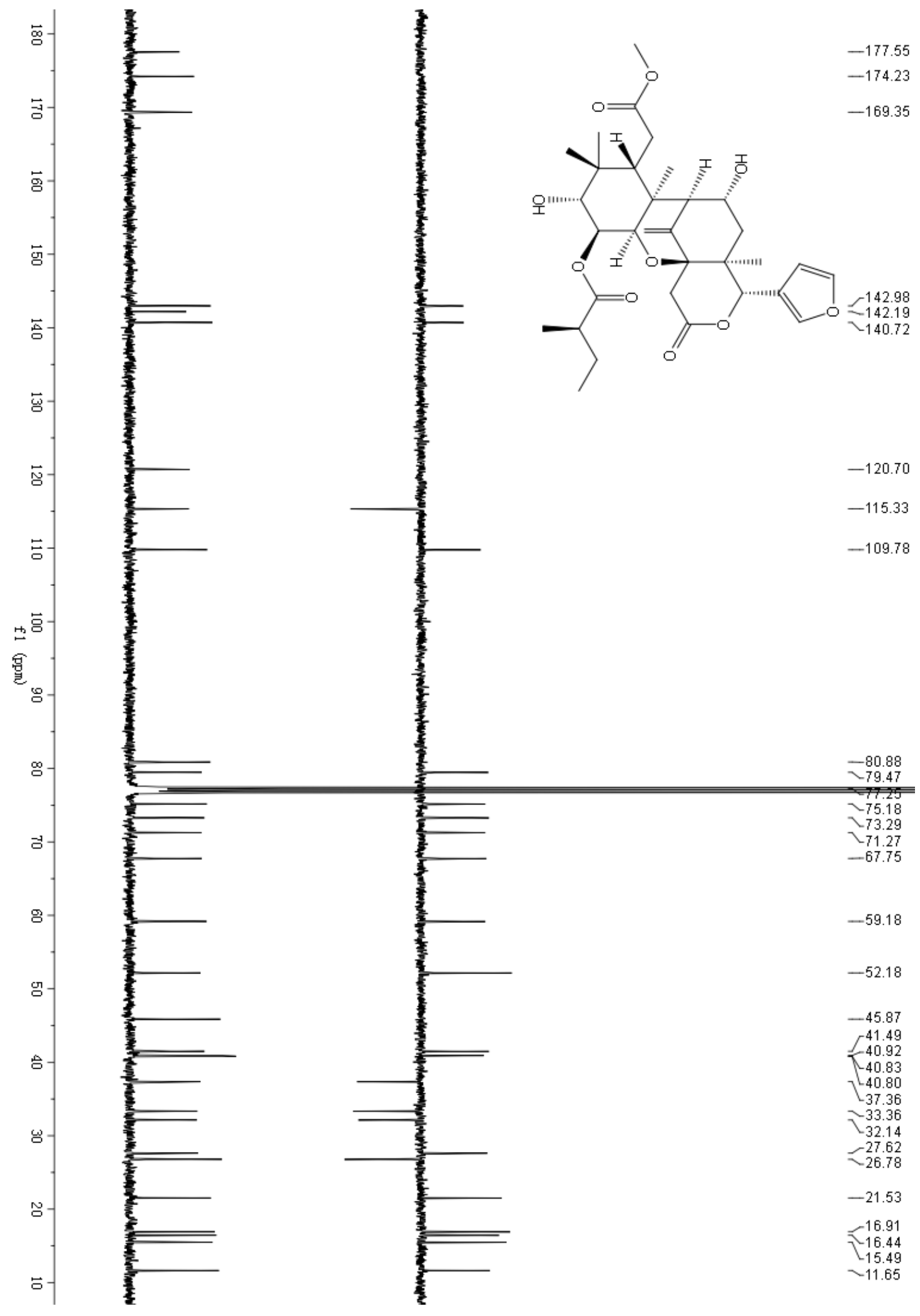


Figure S20. $\mathrm{HSQC}$ spectrum of cibacciferin $\mathrm{B}(3)$ in $\mathrm{CDCl}_{3}$

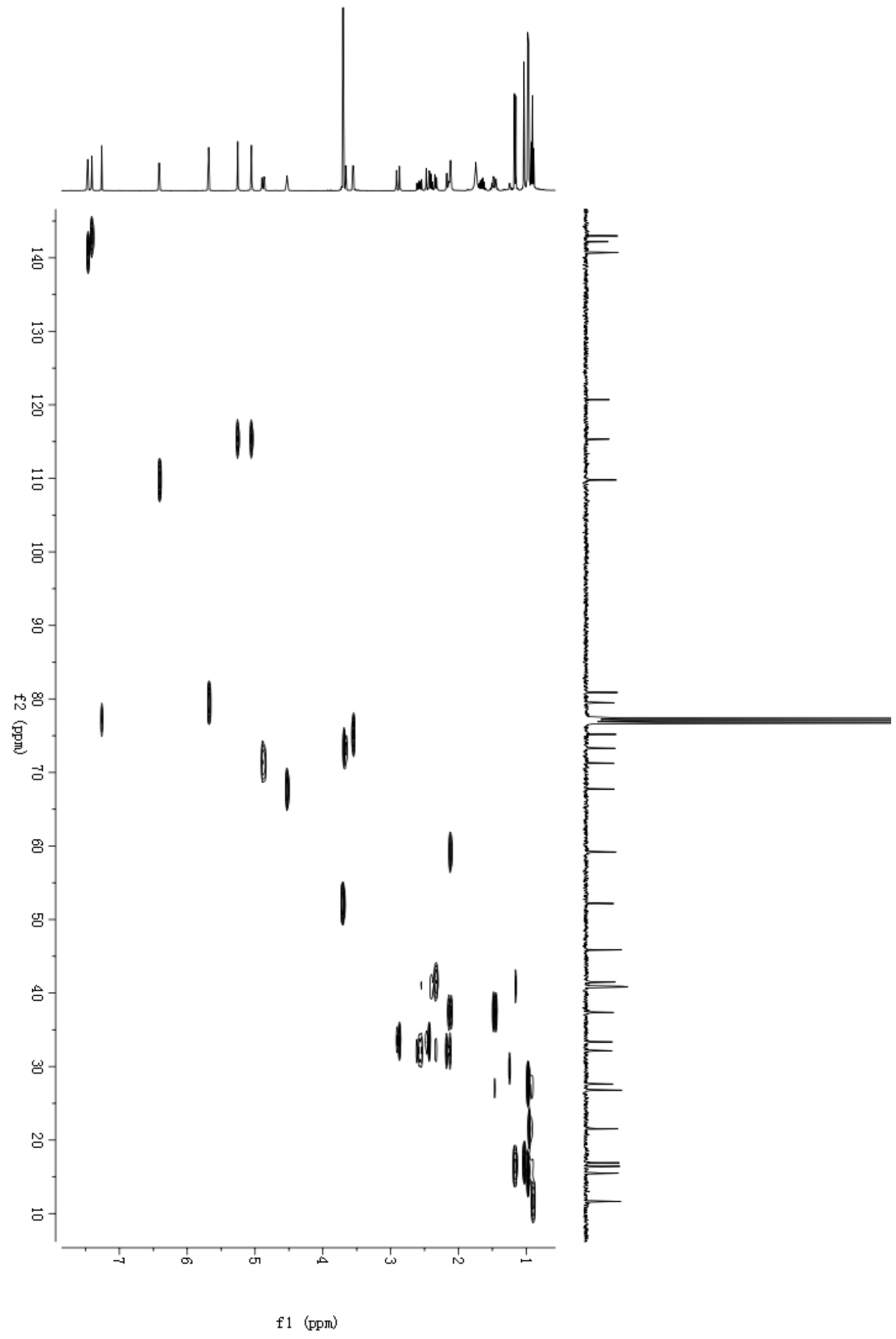


Figure S21. HMBC spectrum of cibacciferin $\mathrm{B}(3)$ in $\mathrm{CDCl}_{3}$

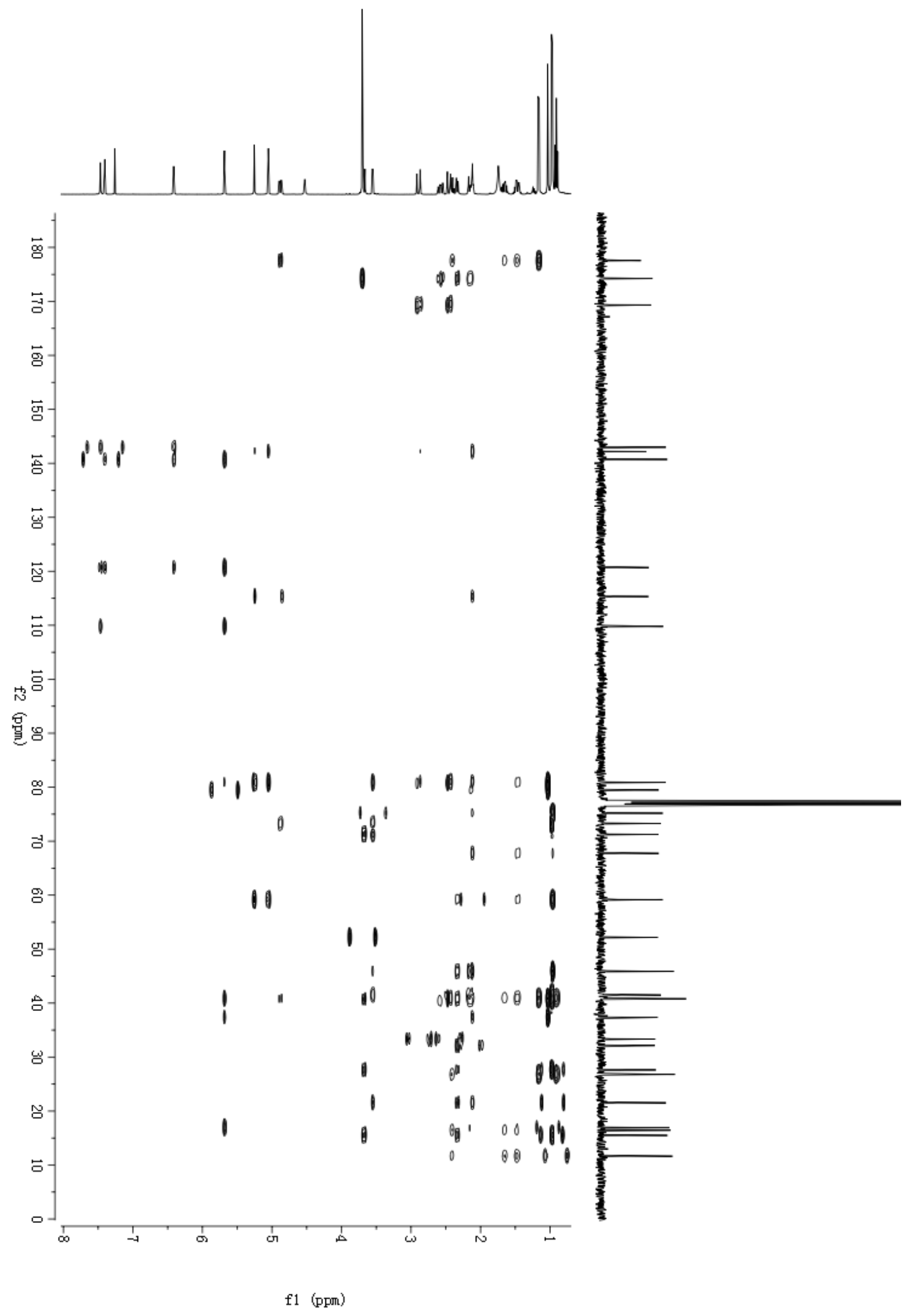


Figure S22. ESI(+)MS spectrum of cibacciferin B (3)

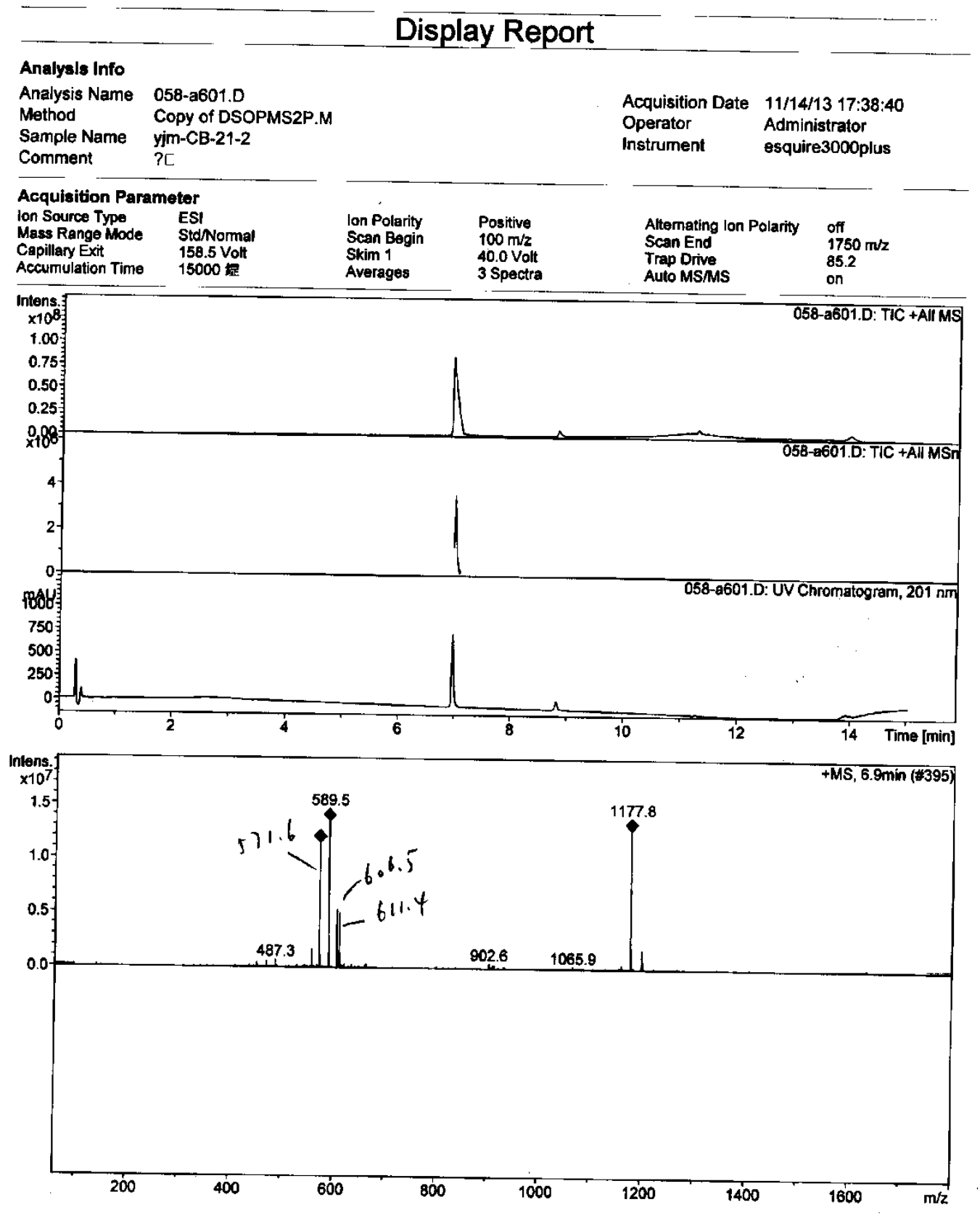


Figure S23. ESI(-)MS spectrum of cibacciferin B (3)

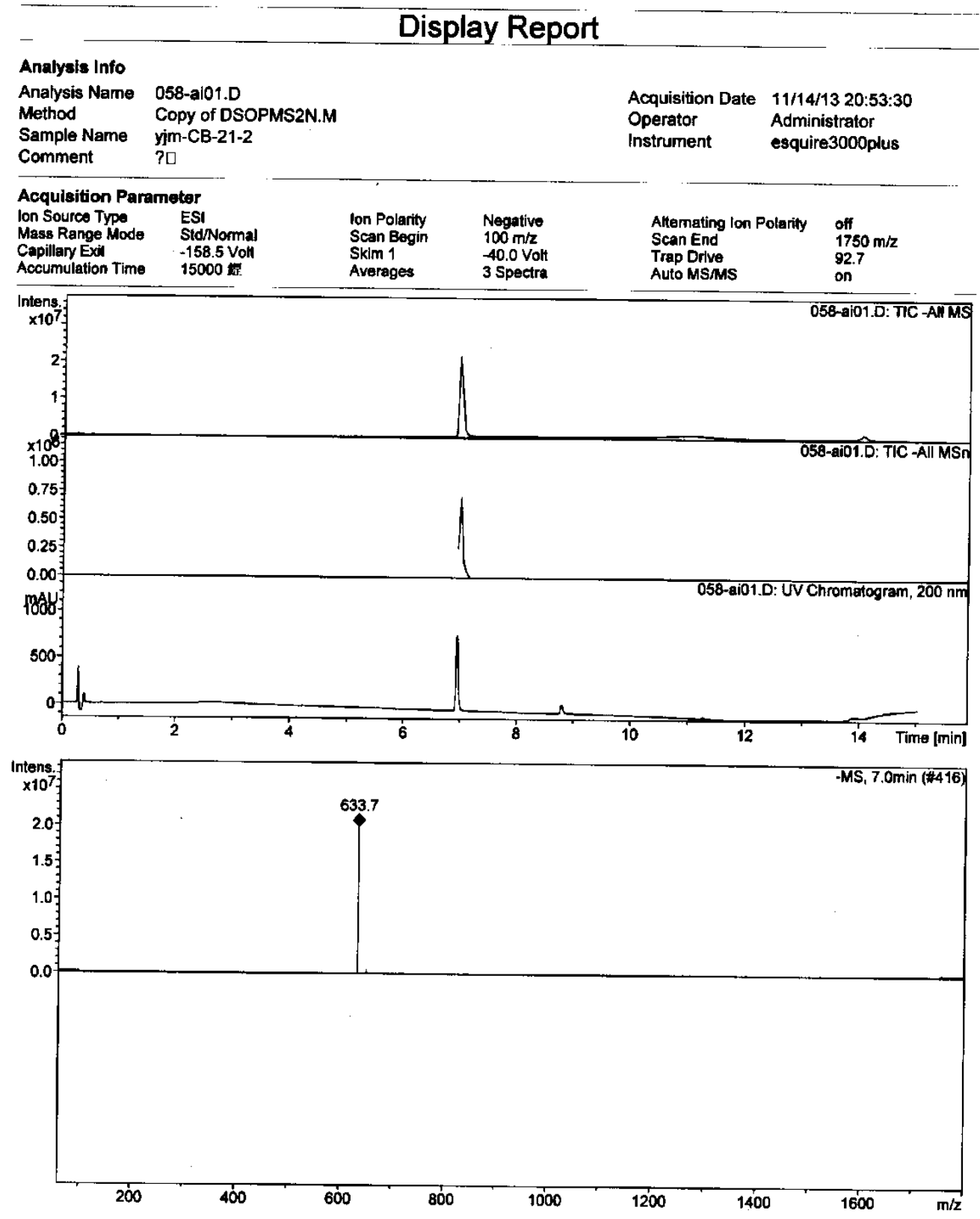


Figure S24. HRESI(+)MS spectrum of cibacciferin B (3)

Elemental Composition Report

Single Mass Analysis

Tolerance $=3.0 \mathrm{PPM} / \mathrm{DBE}: \min =-1.5, \max =50.0$

Element prediction: Off

Number of isotope peaks used for i-FIT $=3$

Monoisotopic Mass, Even Electron lons

249 formula(e) evaluated with 1 results within limits (up to 50 closest results for each mass)

Elements Used:

$\begin{array}{llll}\text { C: } 5-80 & \text { H: } 2-120 & \text { O: } 0-20 & \mathrm{Na}: 0-1\end{array}$

$\begin{array}{llll}\text { C: } 5-80 & \text { H: } 2-120 & \text { O: } 0-20 & \mathrm{Na}: 0-1 \quad \text { LCT PXE KE324 }\end{array}$

19-Nov-2013

16:38:37

CB-21-2_1119 23 (0.495) AM2 (Ar, 10000.0,0.00,1.00); ABS; Cm (21:35)

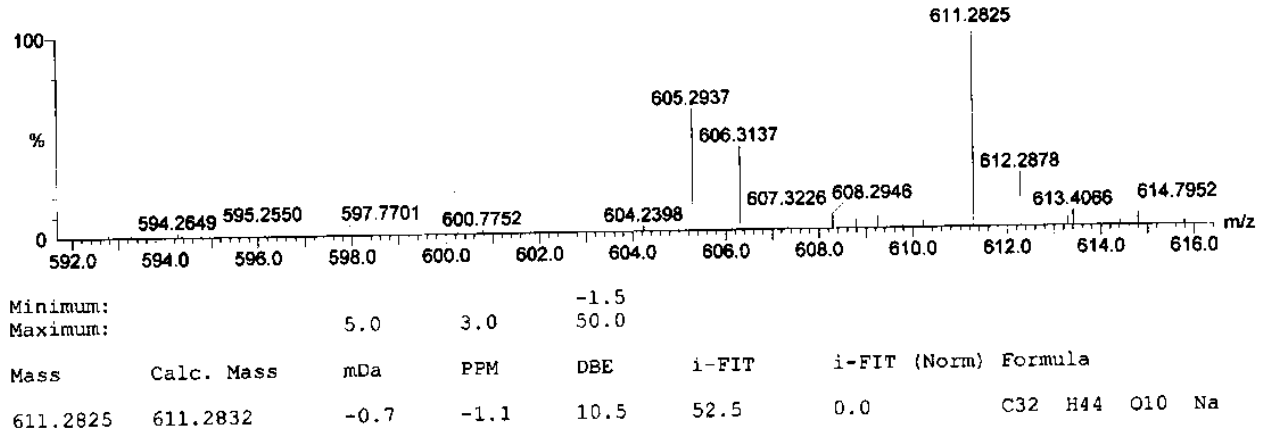


Figure S25. IR spectrum of cibacciferin B (3)

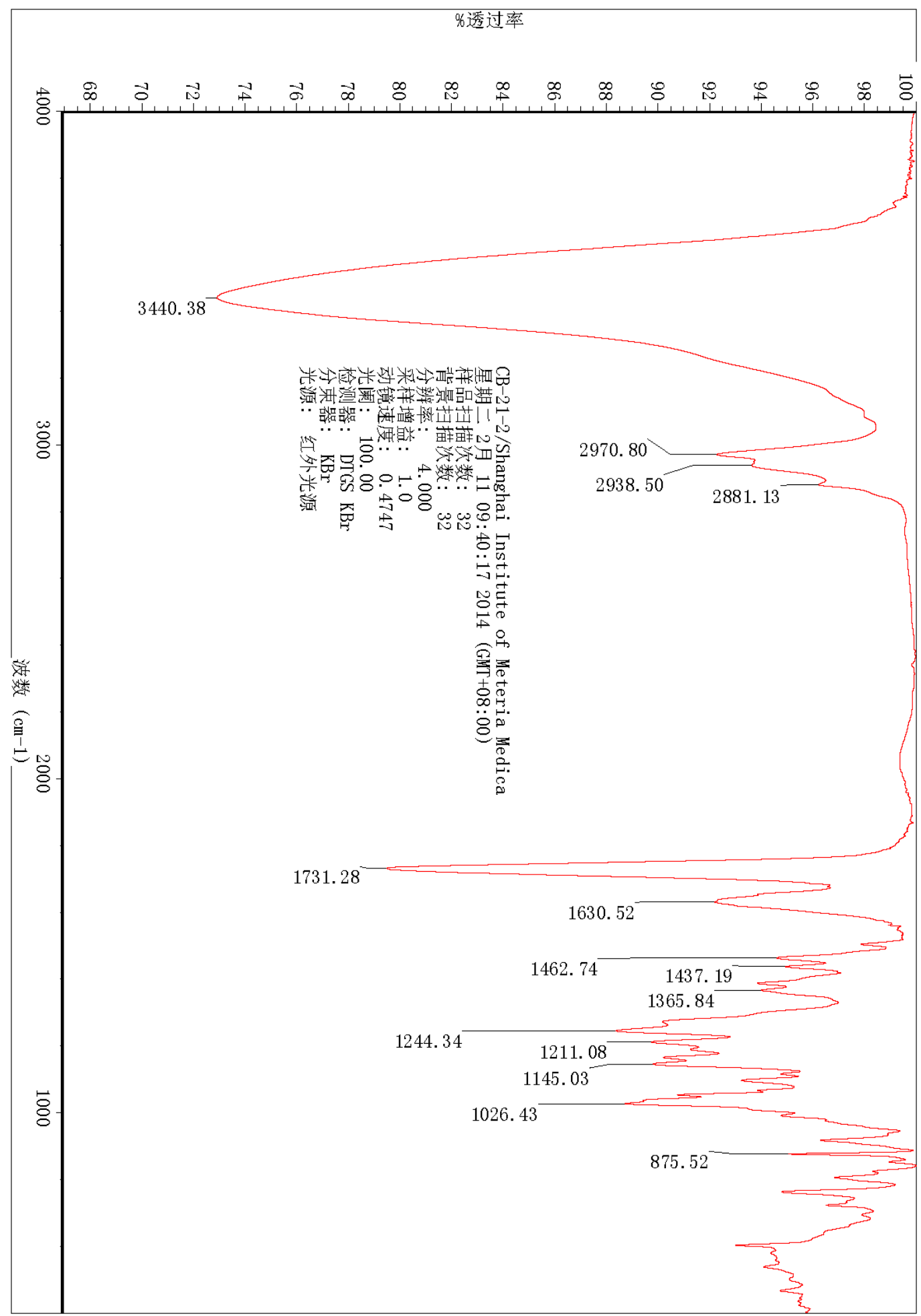


Figure S26. ${ }^{1} \mathrm{H}$ NMR spectrum of $2{ }^{\prime}$-epi-cibacciferin B (4) in $\mathrm{CDCl}_{3}$
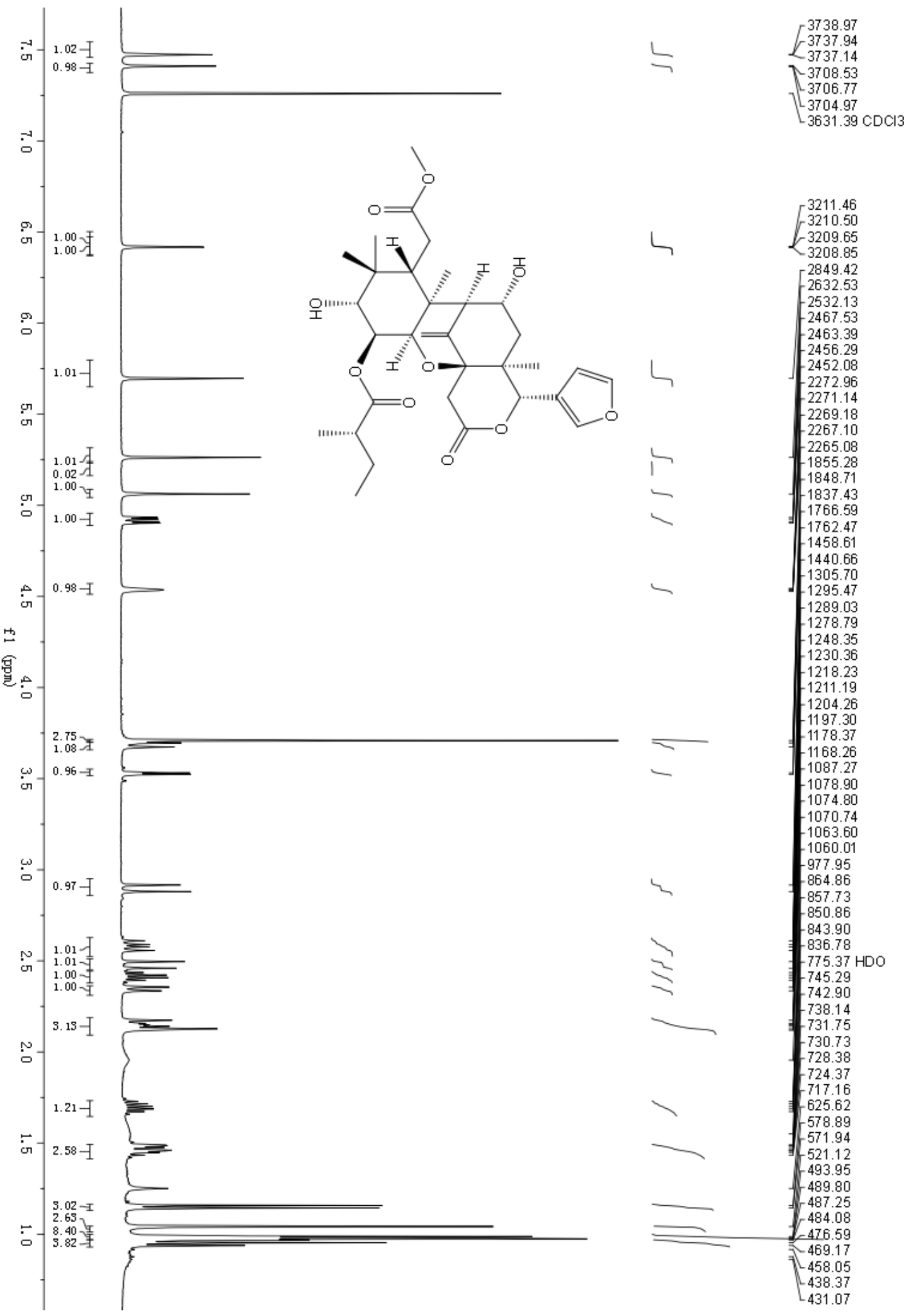
Figure S27. ${ }^{13} \mathrm{C}$ NMR spectrum of $2^{\prime}$-epi-cibacciferin $\mathrm{B}(4)$ in $\mathrm{CDCl}_{3}$

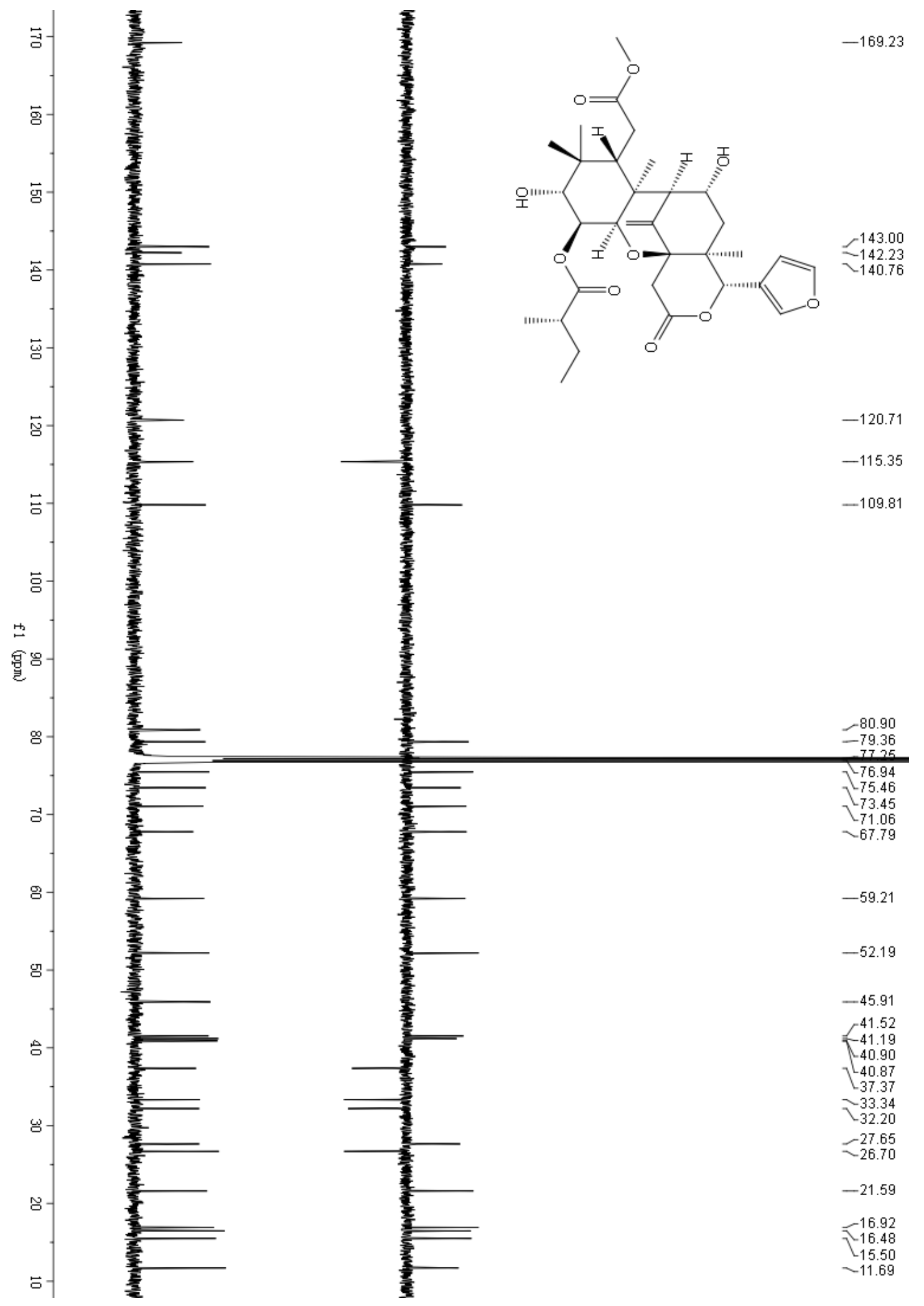


Figure S28. HSQC spectrum of 2'-epi-cibacciferin B (4) in $\mathrm{CDCl}_{3}$

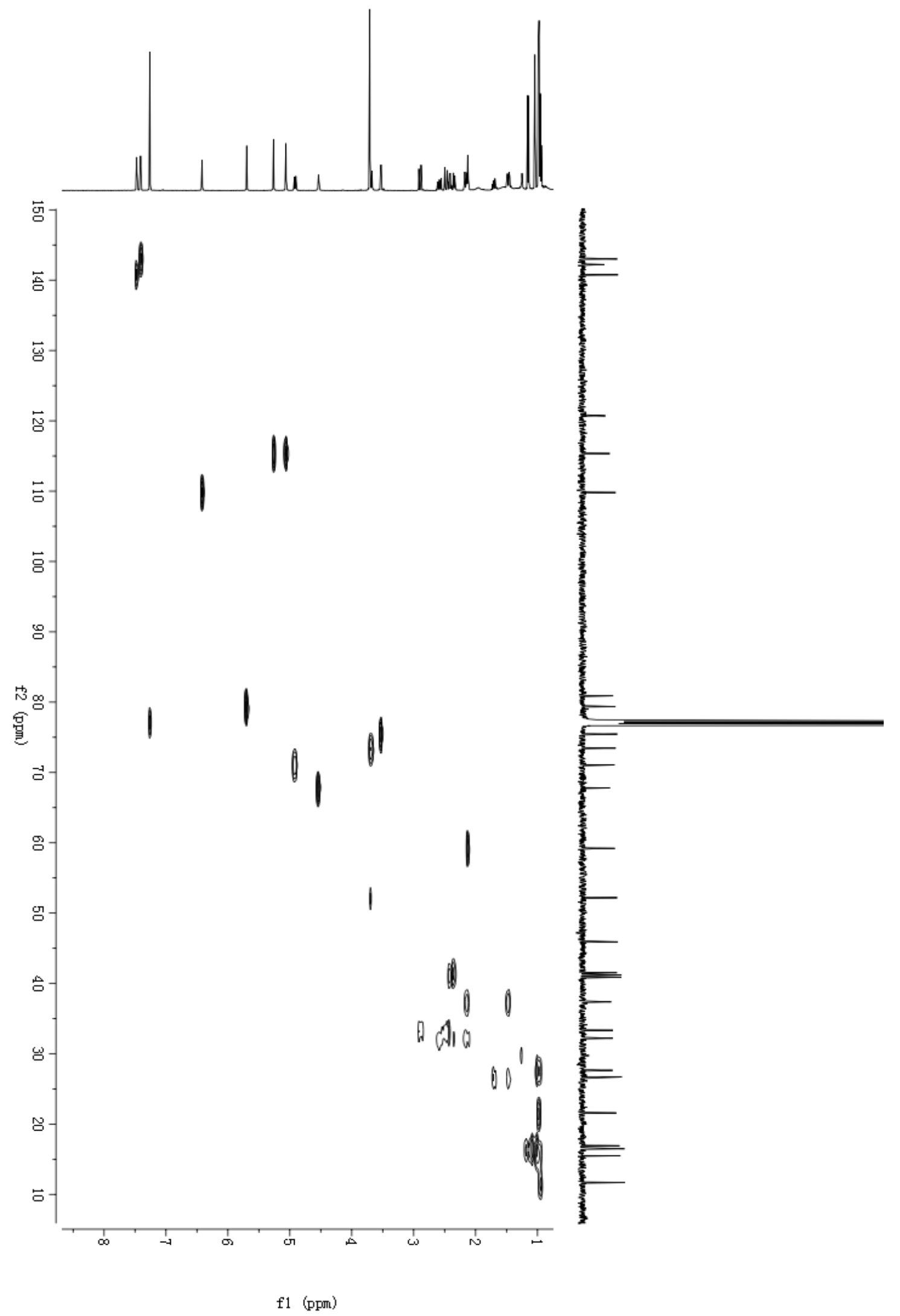


Figure S29. $\mathrm{HMBC}$ spectrum of $2^{\prime}$-epi-cibacciferin $\mathrm{B}(4)$ in $\mathrm{CDCl}_{3}$

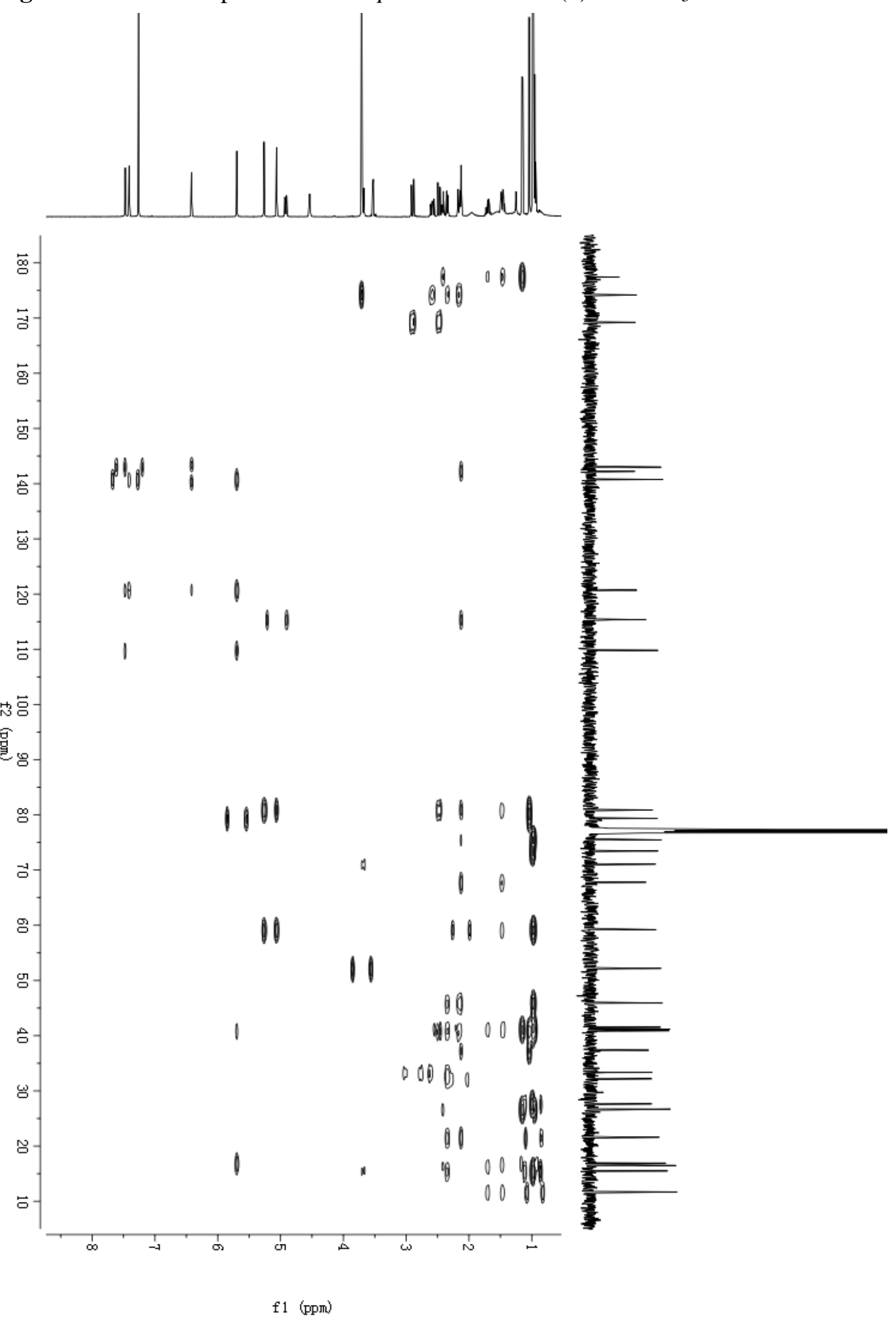

39 
Figure S30. ESI(+)MS spectrum of 2'-epi-cibacciferin B (4)

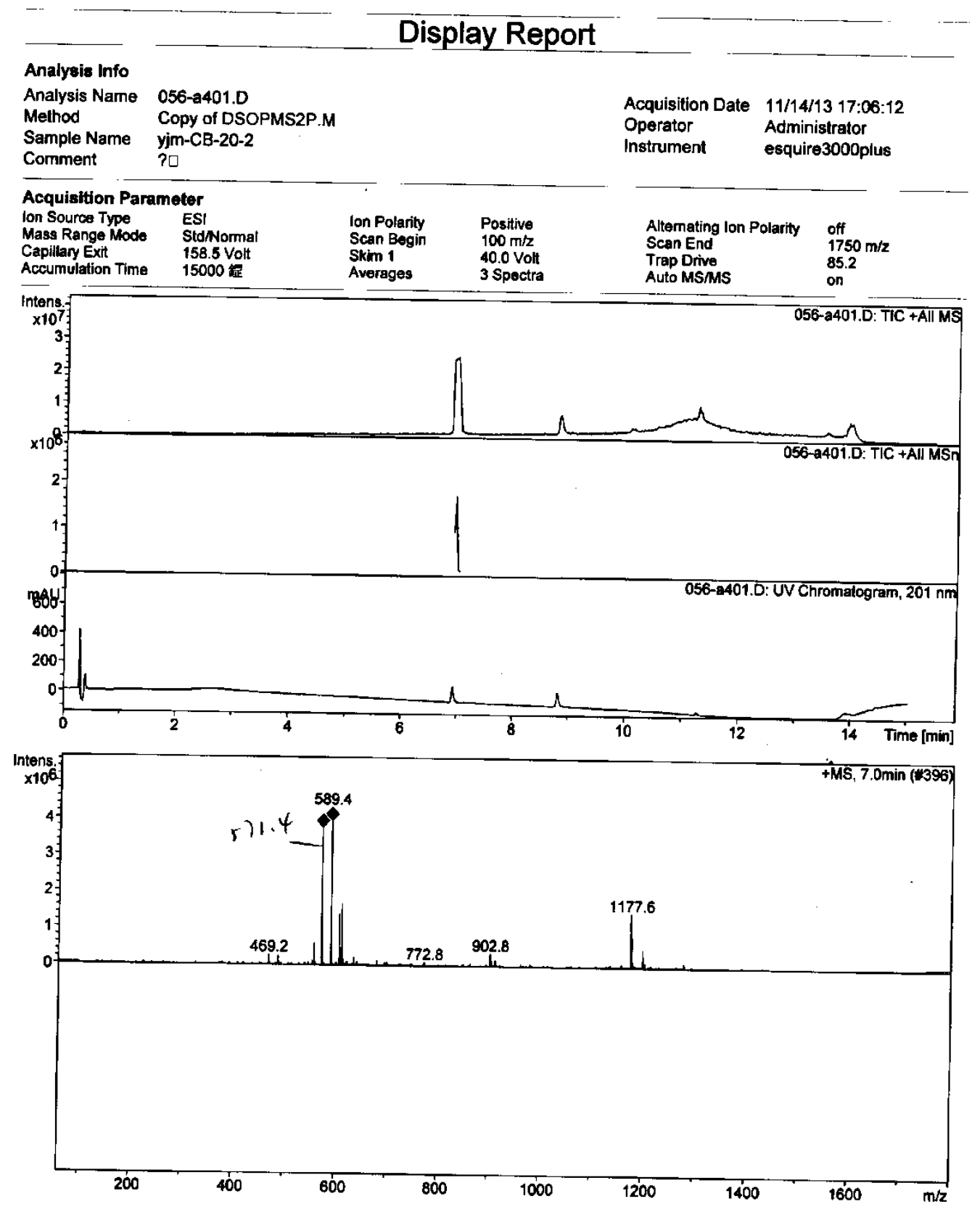


Figure S31. ESI(-)MS spectrum of 2'-epi-cibacciferin B (4)

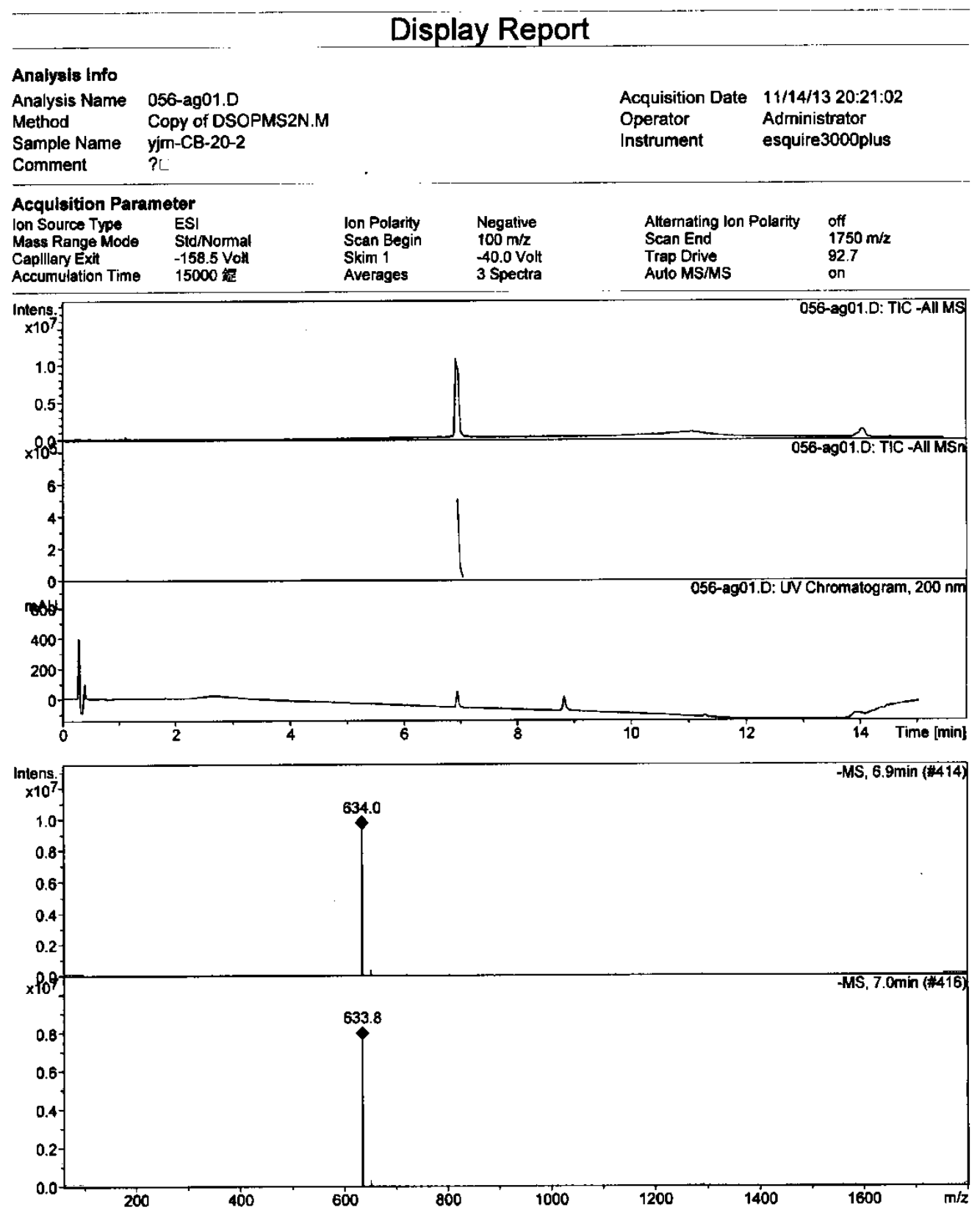


Figure S32. HRESI(+)MS spectrum of 2'-epi-cibacciferin B (4)

\section{Elemental Composition Report}

\section{Single Mass Analysis}

Tolerance $=3.0$ PPM / DBE: $\min =-1.5, \max =50.0$

Element prediction: Off

Number of isotope peaks used for i-FIT $=3$

Monoisolopic Mass, Even Electron lons

249 formula(e)

$\begin{array}{lll}\text { C: } 5-80 & \text { H: } 2-120 & \text { O: } 0-20 \quad \mathrm{Na}: 0-1\end{array}$

CB-20-2

LCT PXE KE324

19-Nov-2013

16:33:58

1: TOF MS ES+

CB-20-2_1119 32 (0.704) AM2 (Ar, 10000.0.0.00,1.00); ABS; Cm (21:33)

$4.77 \mathrm{e}+003$

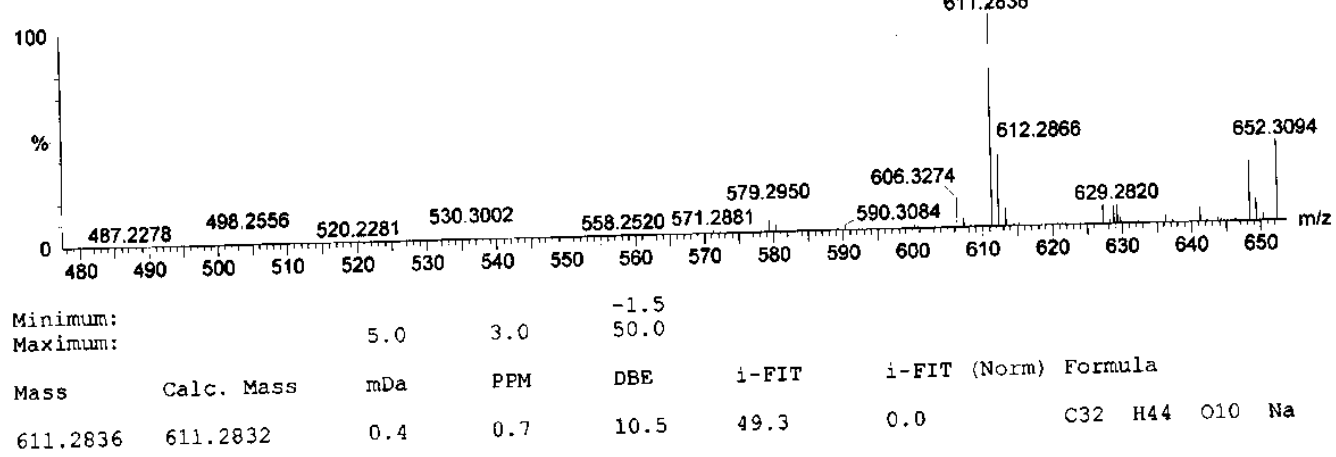


Figure S33. IR spectrum of 2'-epi-cibacciferin B (4)

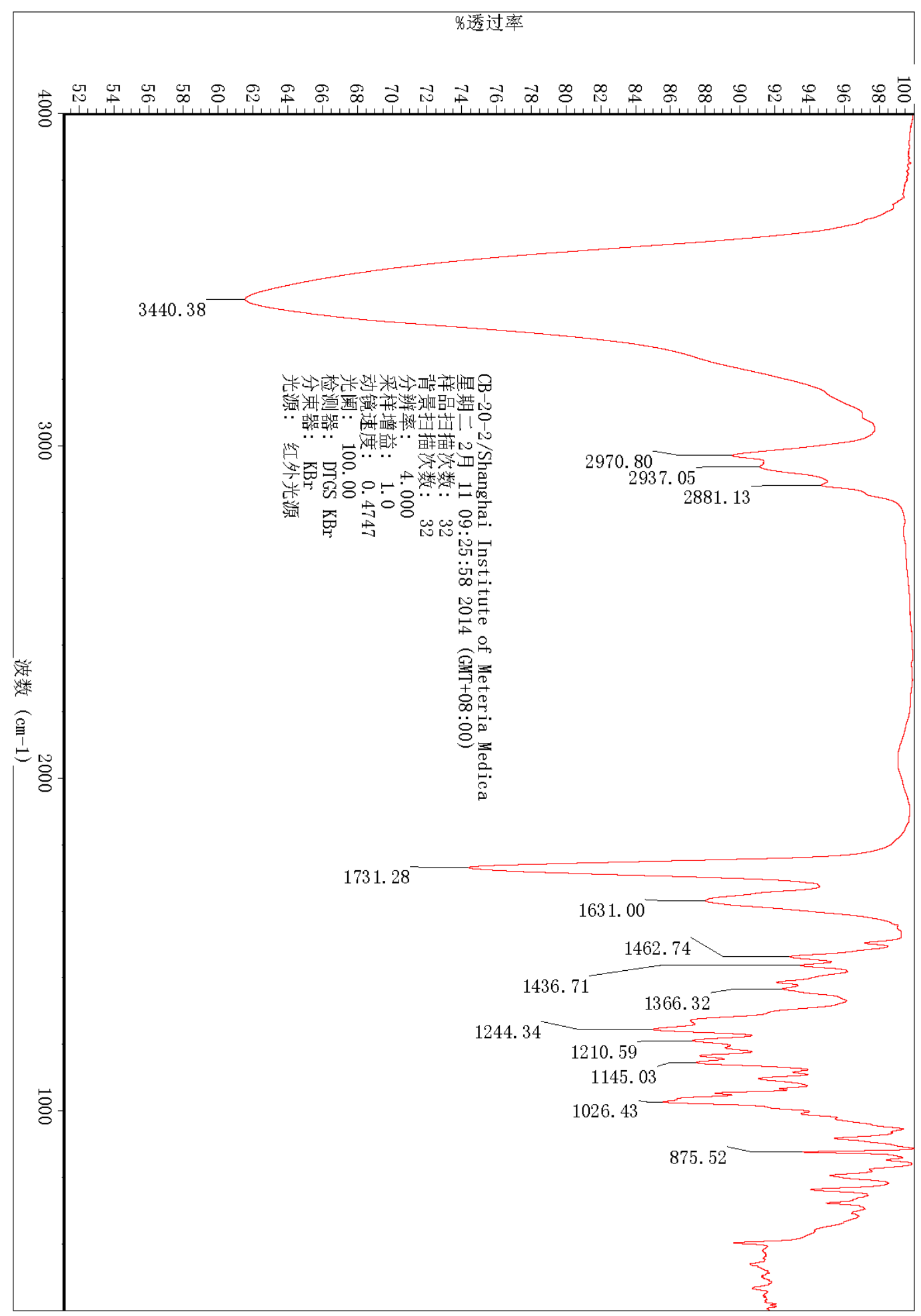


Figure S34. ${ }^{1} \mathrm{H}$ NMR spectrum of cibacciferin $\mathrm{C}(5)$ in $\mathrm{CDCl}_{3}$
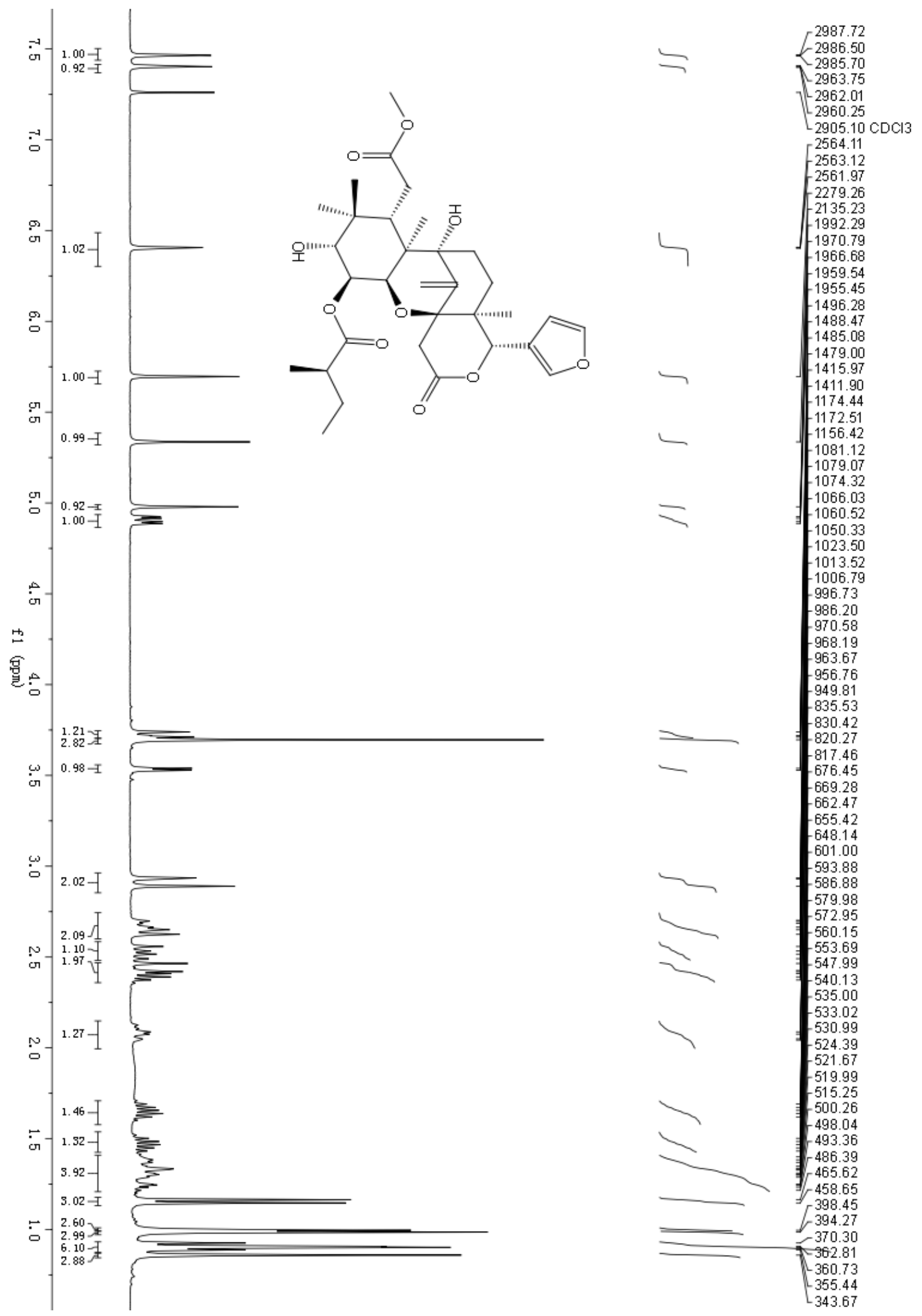
Figure S35. ${ }^{13} \mathrm{C}$ NMR spectrum of cibacciferin $\mathrm{C}(\mathbf{5})$ in $\mathrm{CDCl}_{3}$

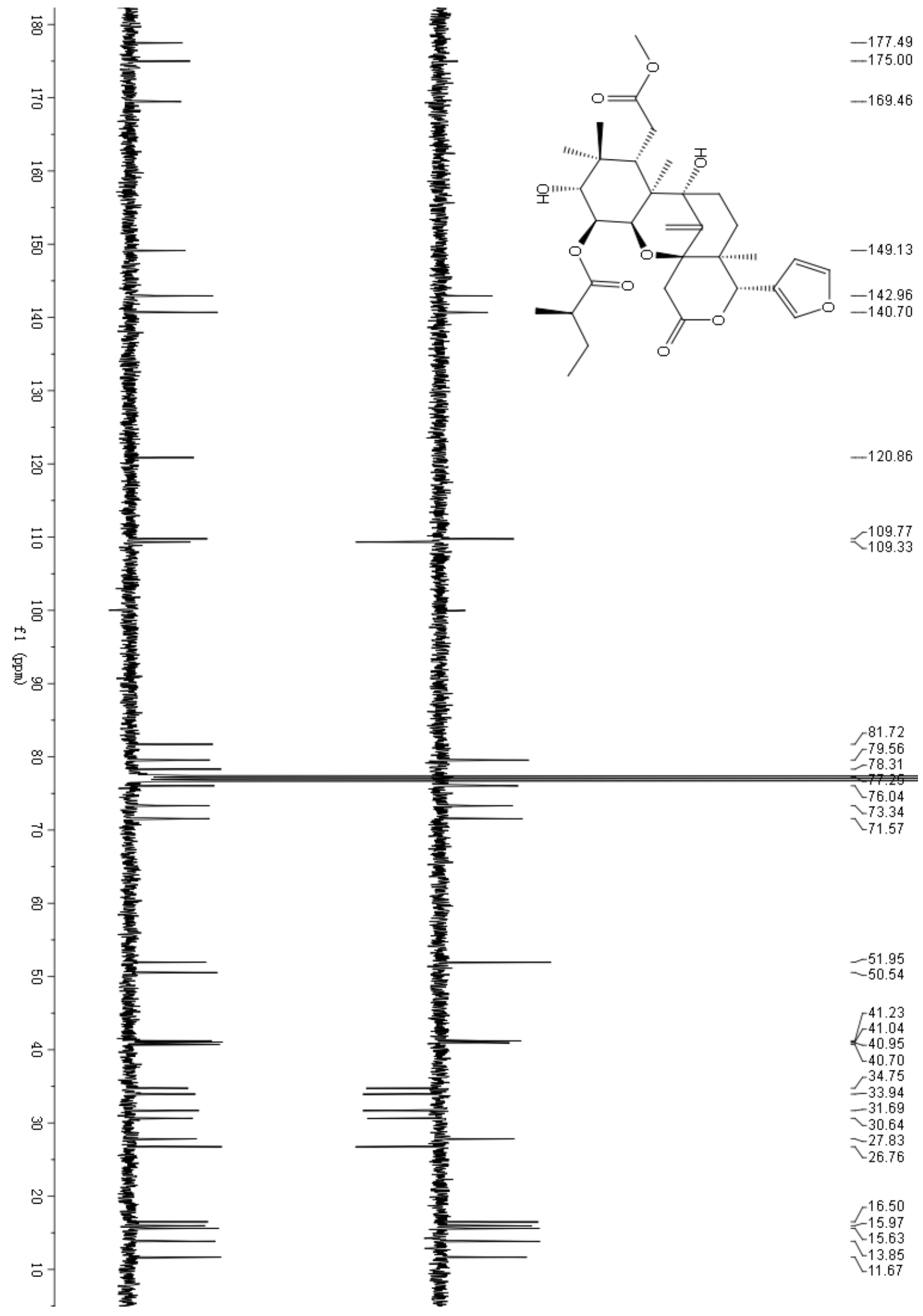


Figure S36. HSQC spectrum of cibacciferin $\mathrm{C}(\mathbf{5})$ in $\mathrm{CDCl}_{3}$

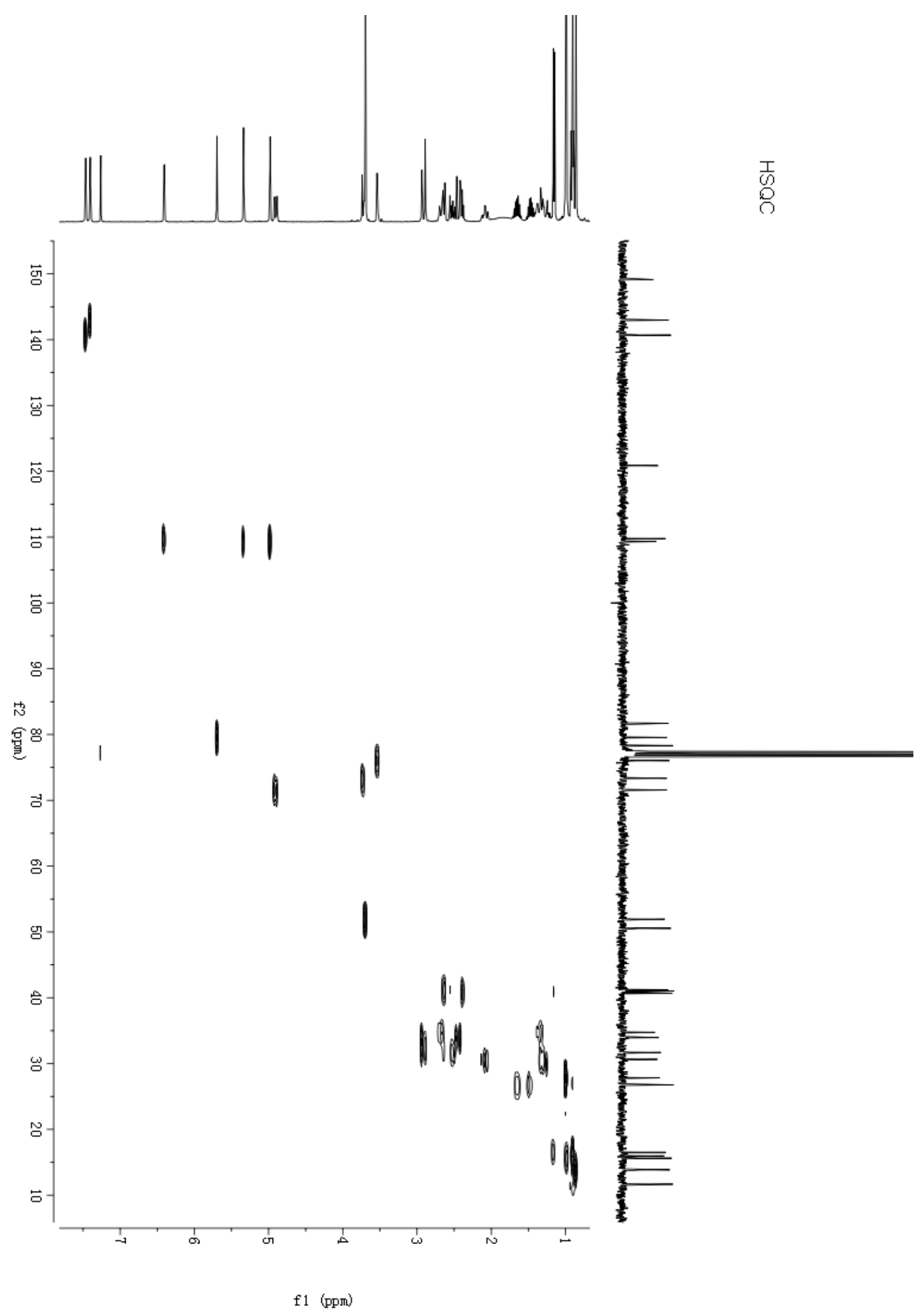

46 
Figure S37. HMBC spectrum of cibacciferin C (5) in $\mathrm{CDCl}_{3}$

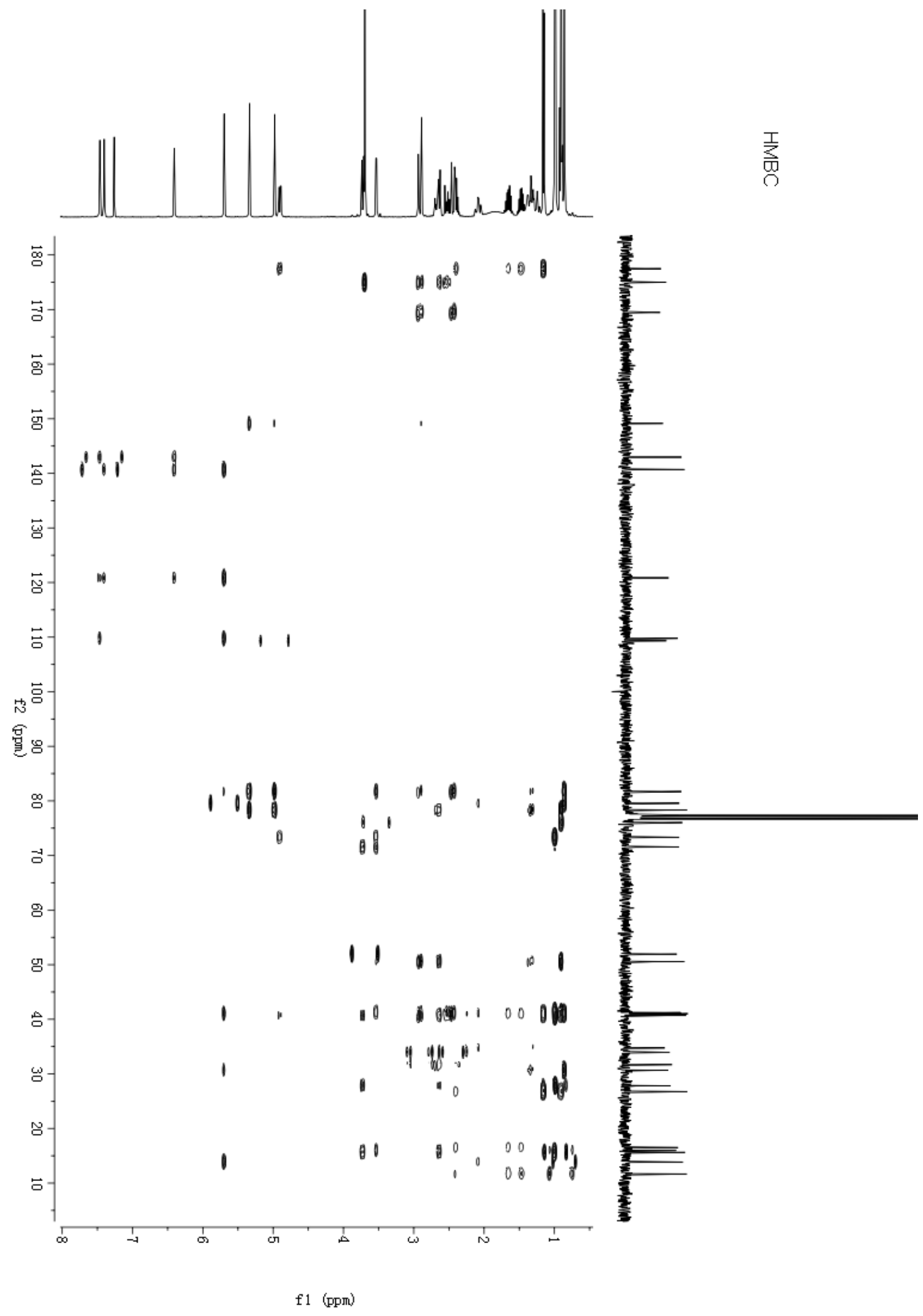


Figure S38. ESI(+)MS spectrum of cibacciferin C (5)

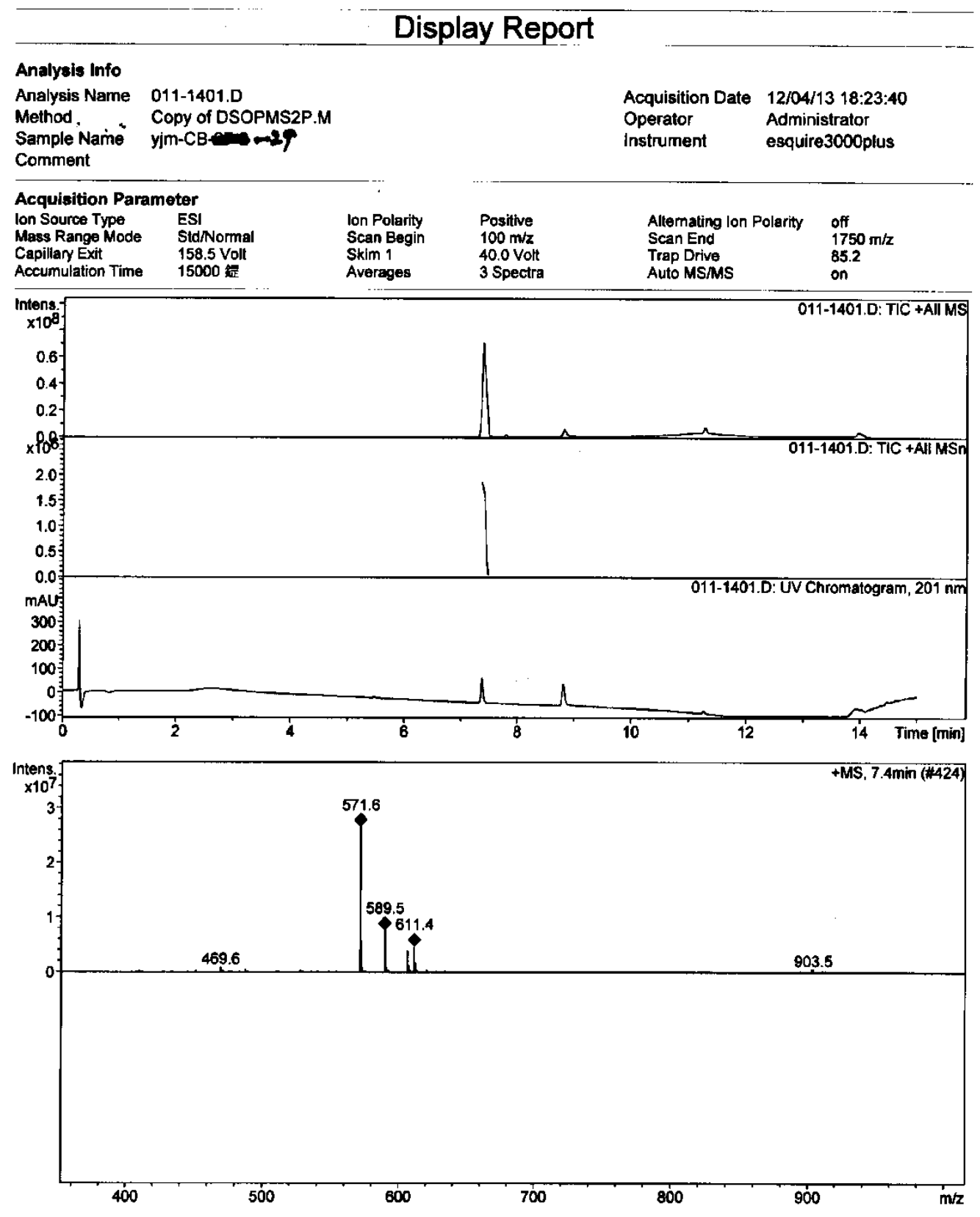


Figure S39. ESI(-)MS spectrum of cibacciferin C (5)

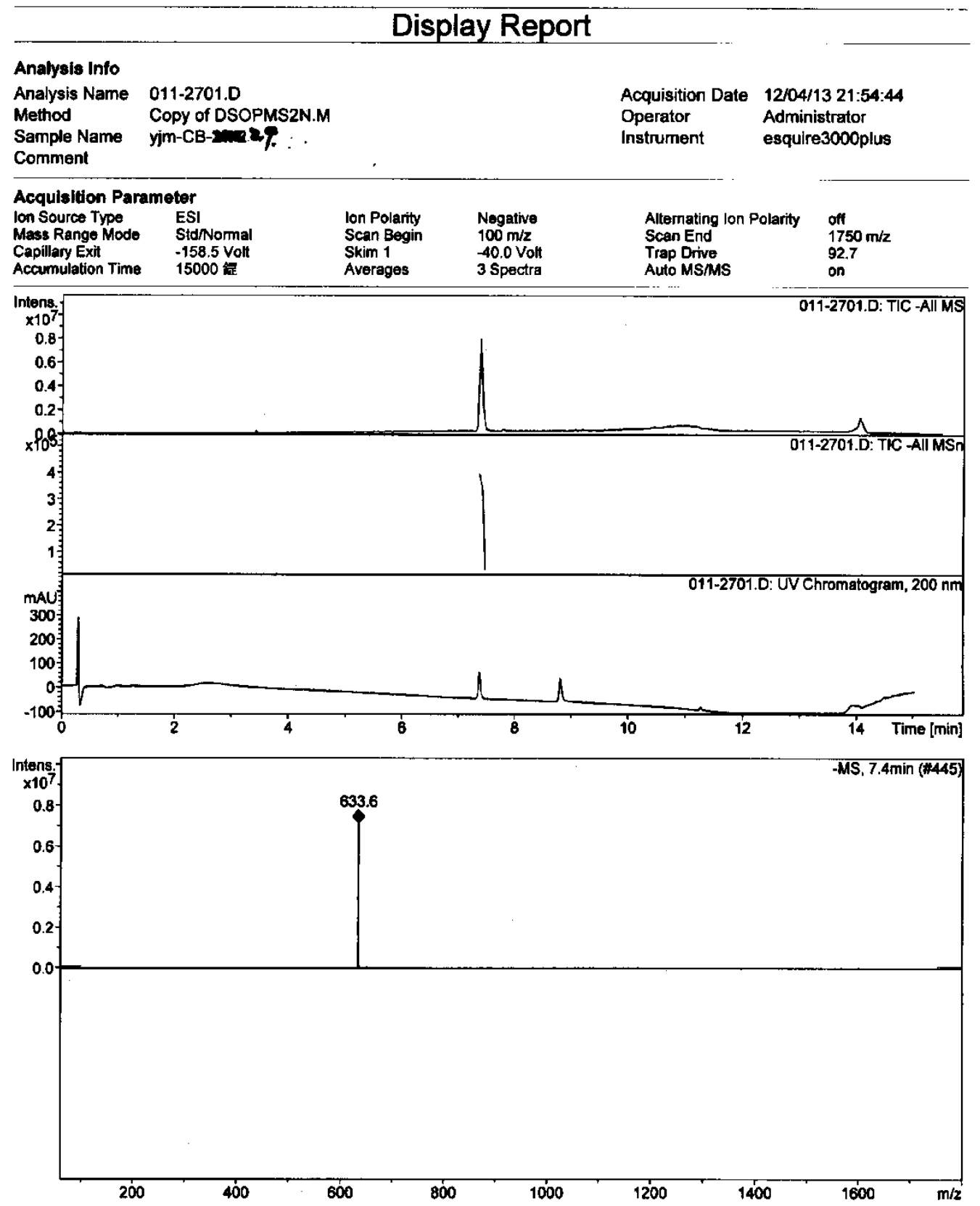


Figure S40. HRESI(+)MS spectrum of cibacciferin C (5)

Elemental Composition Report

Page 1

Single Mass Analysis

Tolerance $=4.0$ PPM \& DBE: $\min =-1.5, \max =50.0$

Element prediction: Off

Number of isotope peaks used for $\mathrm{i}-\mathrm{FIT}=3$

Monoisotopic Mass, Even Electron lons

238 formula(e) evaluated with 1 results within limits (up to 50 closest results for each mass)

Elements Used:

$\begin{array}{llll}\text { C: } 5-80 & \text { H: } 2-120 & \text { O: } 0-20 \quad \mathrm{Na}: 0-1\end{array}$

CB-29 LCT PXE KE324

05-Dec-2013

16:58:34

CB-29_1205 17 (0.373) AM2 (Ar, 10000.0,0.00, 1.00); ABS; Cm (17:36)

100

589.3020

$1.38 \mathrm{e}+004$

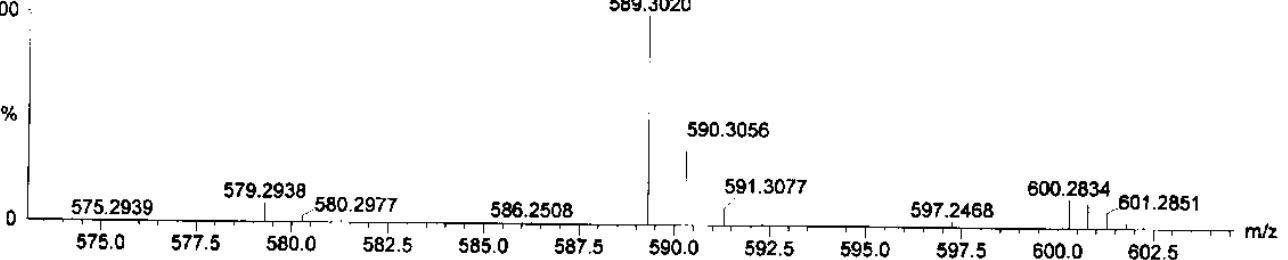

Minimum:

$5.0 \quad 4.0$

Calc. Mass ma

$\begin{array}{llllllllll}589.3020 & 589.3013 & 0.7 & 1.2 & 10.5 & 88.1 & 0.0 & \text { C32 H45 } & 010\end{array}$ 
Figure S41. IR spectrum of cibacciferin C (5)

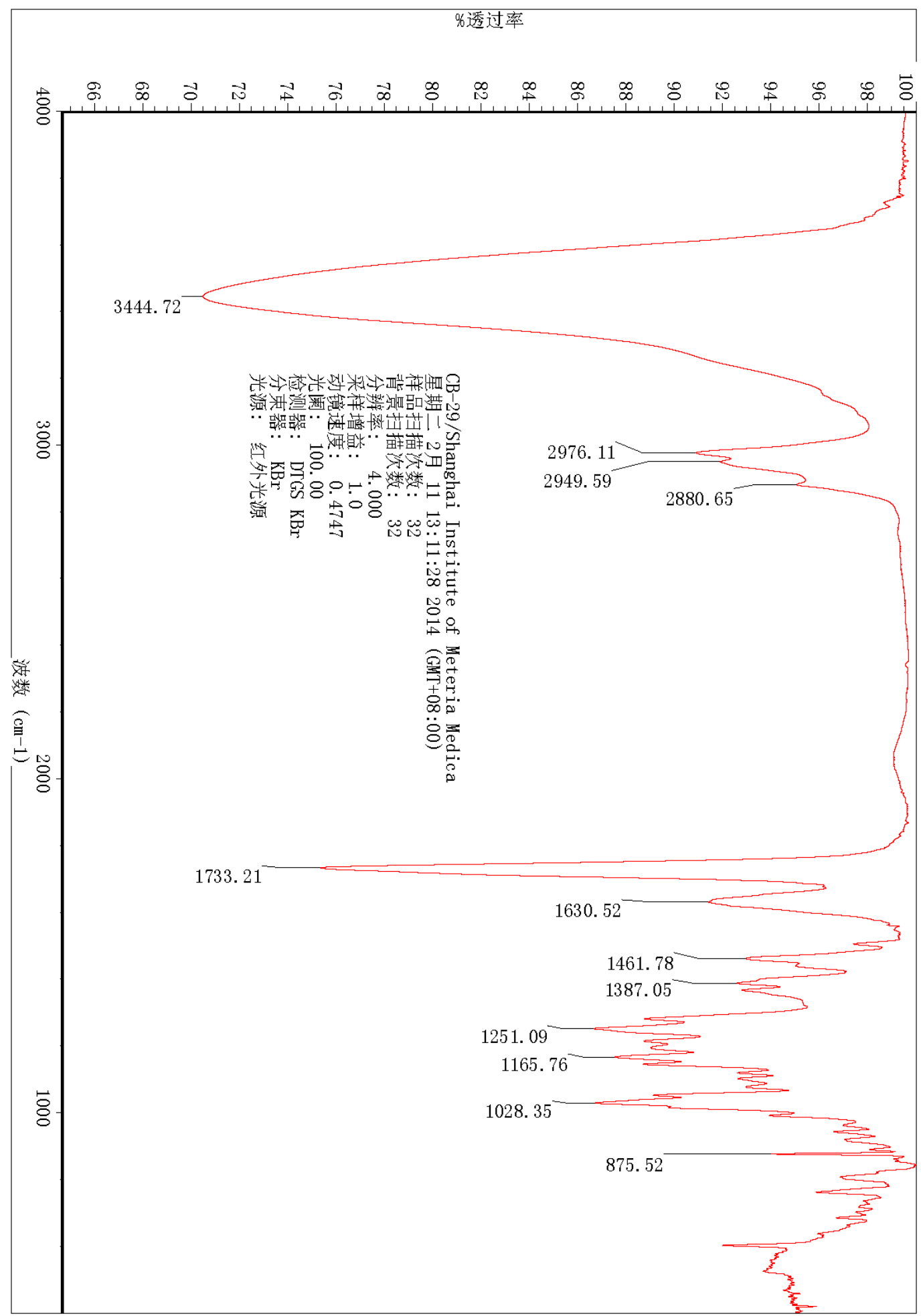


Figure S42. ${ }^{1} \mathrm{H}$ NMR spectrum of $2{ }^{\prime}$-epi-cibacciferin $\mathrm{C}(\mathbf{6})$ in $\mathrm{CDCl}_{3}$

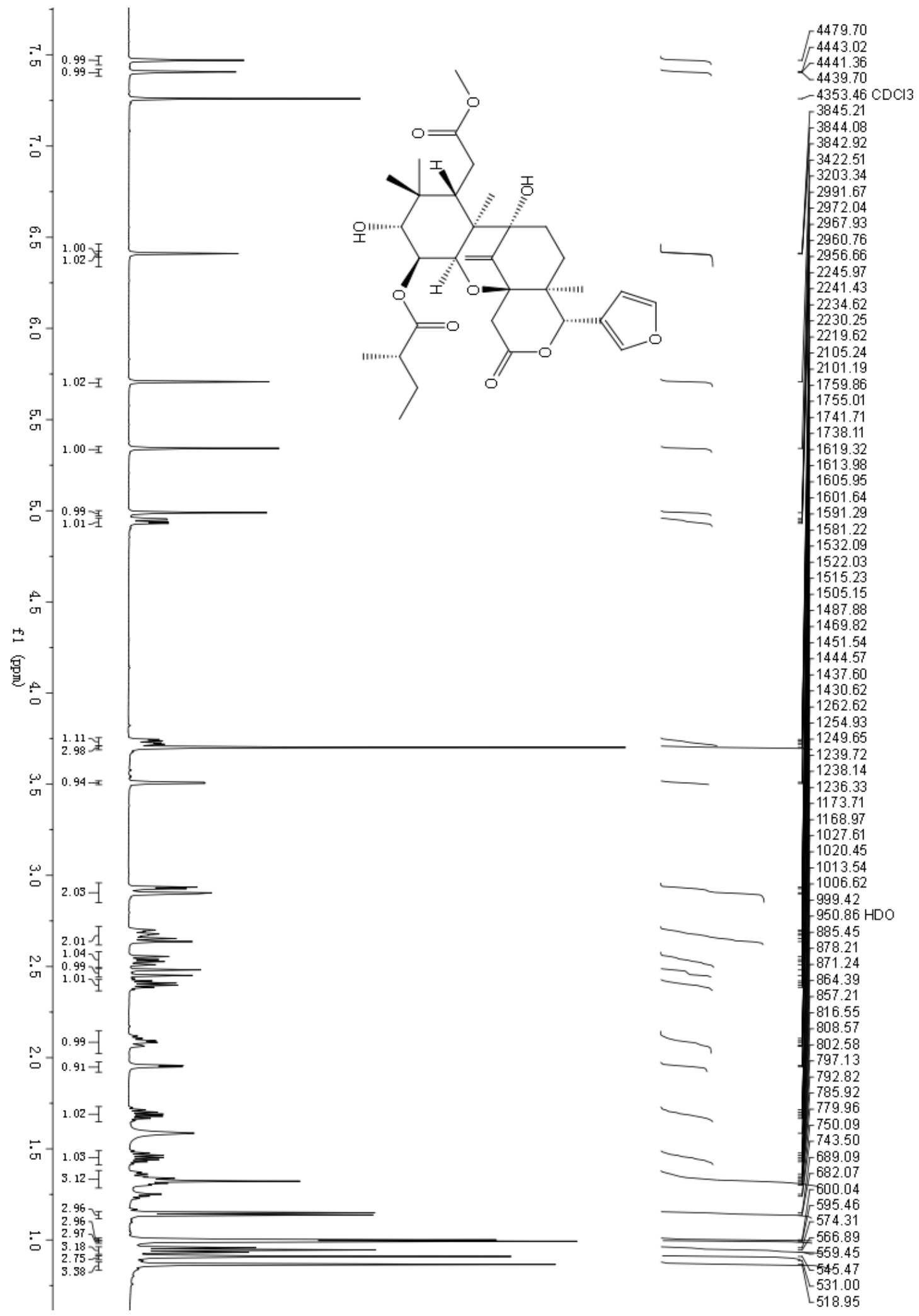


Figure S43. ${ }^{13} \mathrm{C}$ NMR spectrum of $2^{\prime}$-epi-cibacciferin $\mathrm{C}(\mathbf{6})$ in $\mathrm{CDCl}_{3}$

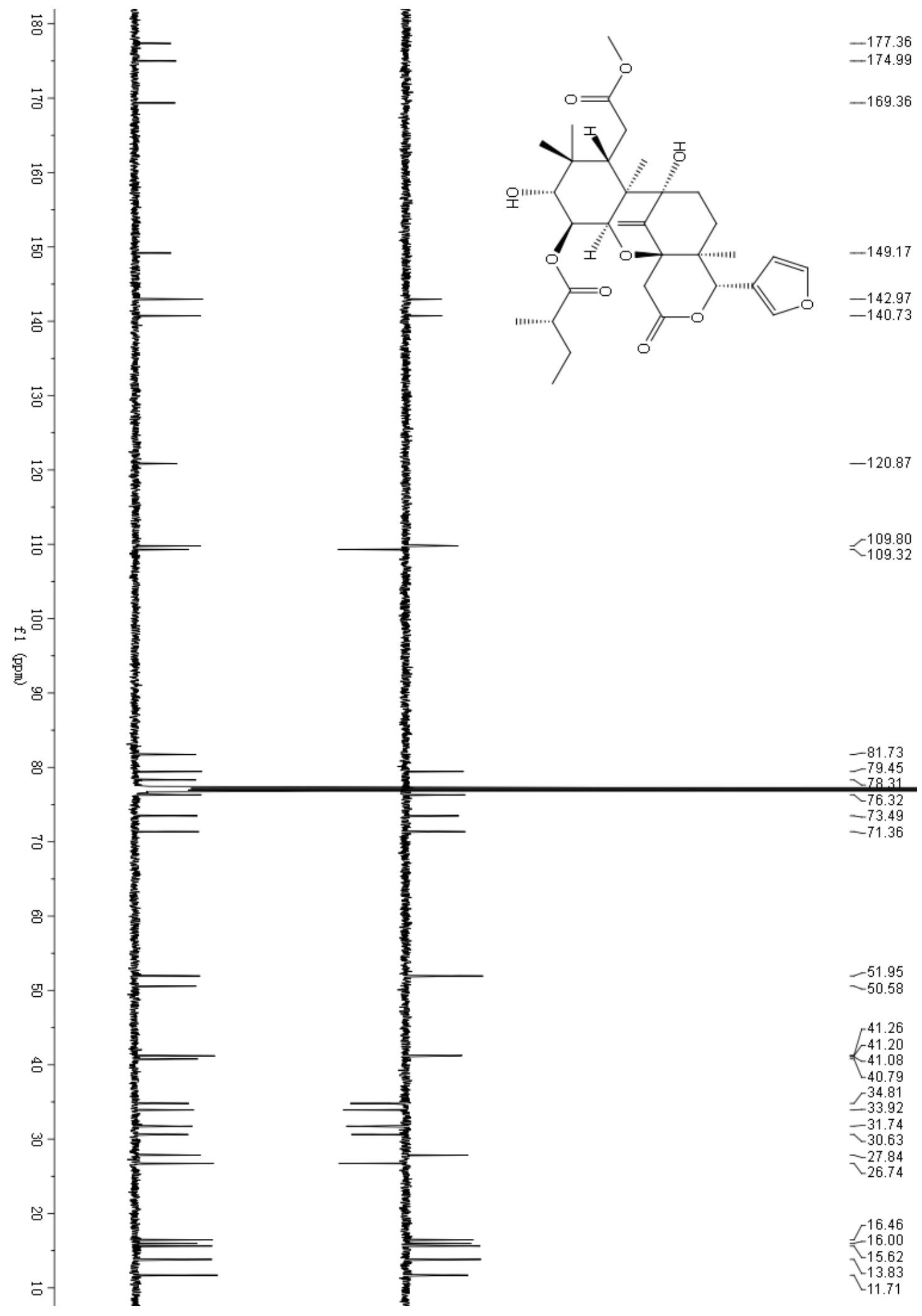


Figure S44. HSQC spectrum of 2'-epi-cibacciferin C (6) in $\mathrm{CDCl}_{3}$

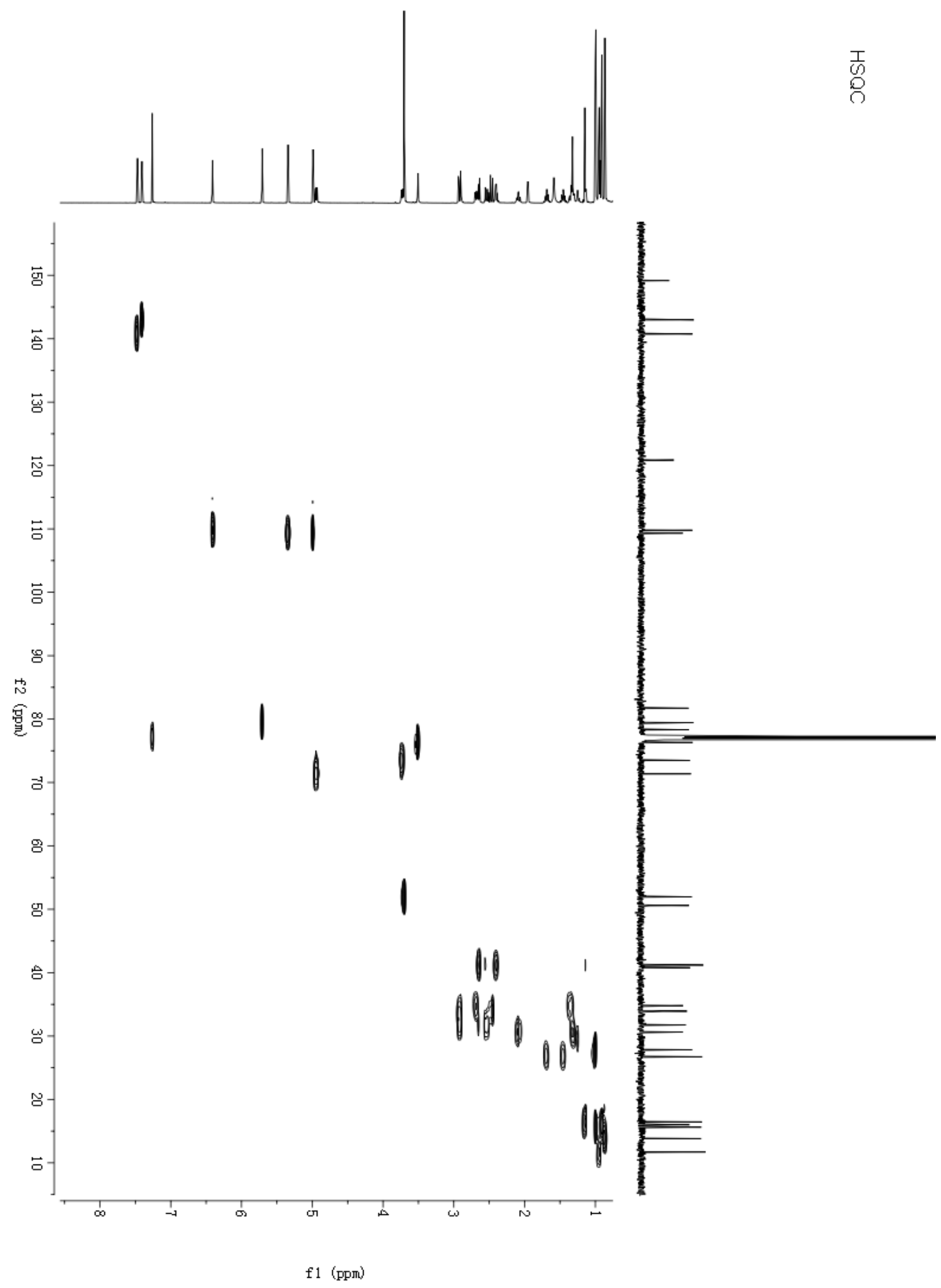


Figure S45. HMBC spectrum of 2 '-epi-cibacciferin $\mathrm{C}(\mathbf{6})$ in $\mathrm{CDCl}_{3}$

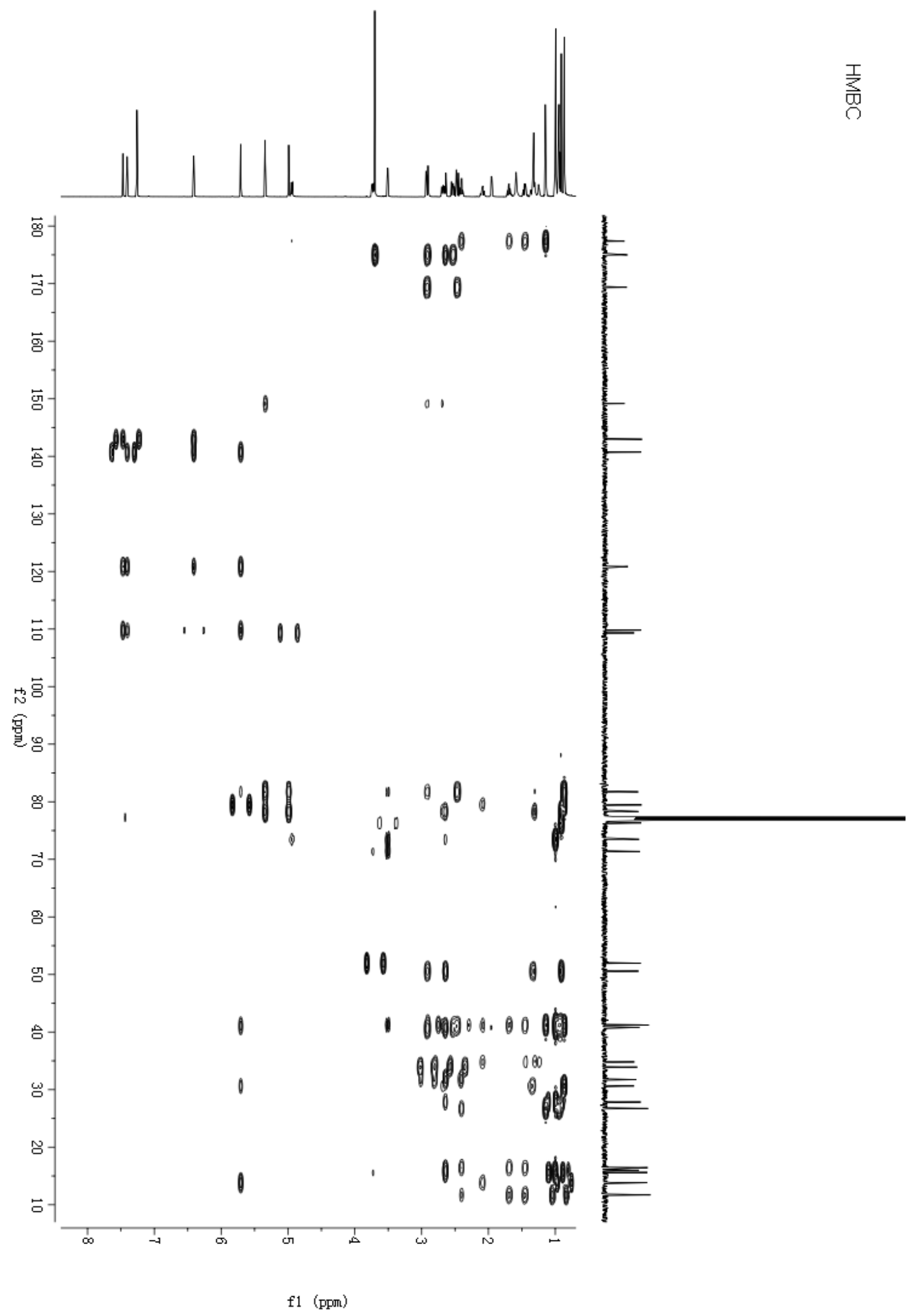


Figure S46. ESI(+)MS spectrum of 2'-epi-cibacciferin C (6)

\section{Display Report}
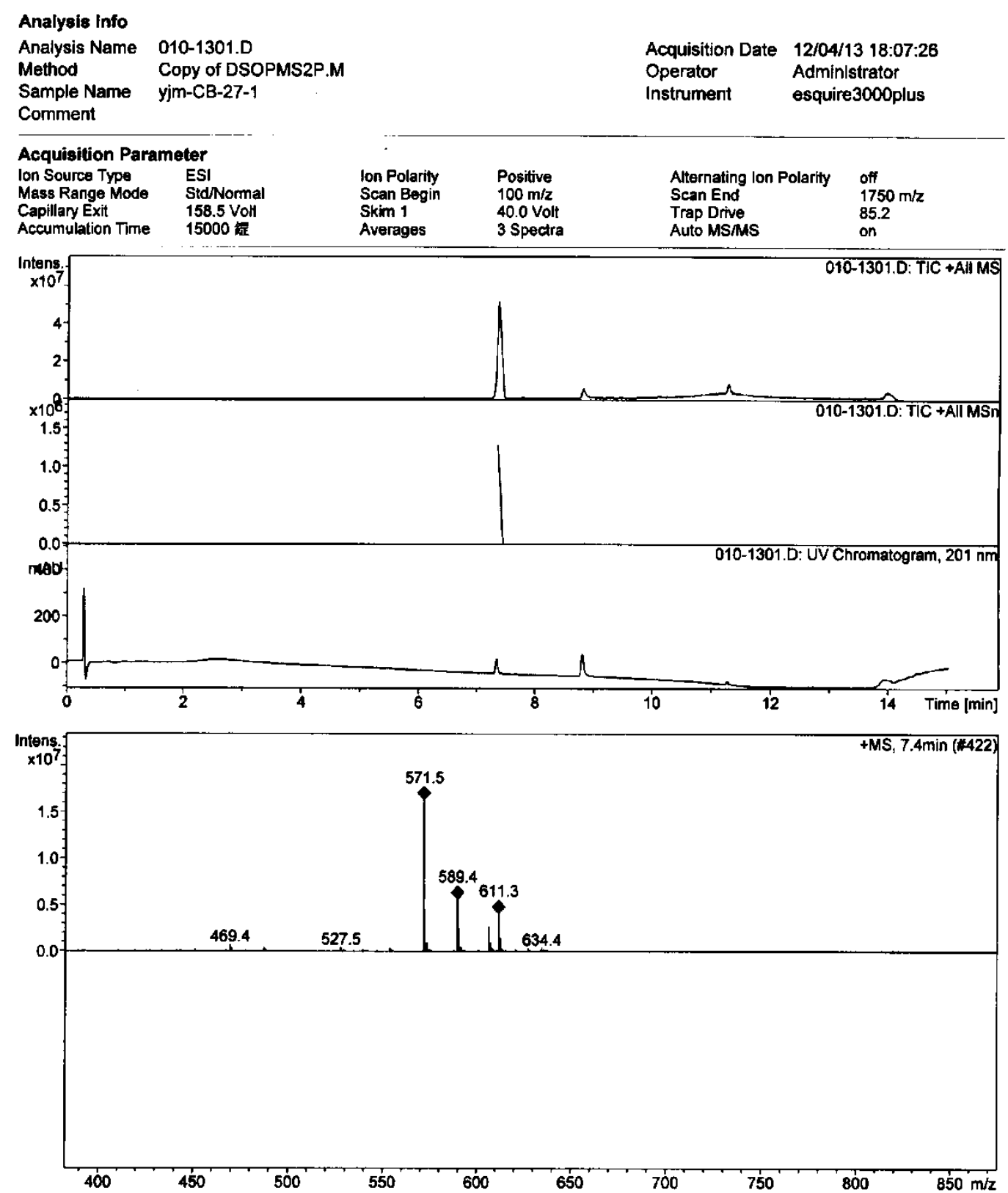
Figure S47. ESI(-)MS spectrum of 2'-epi-cibacciferin C (6)

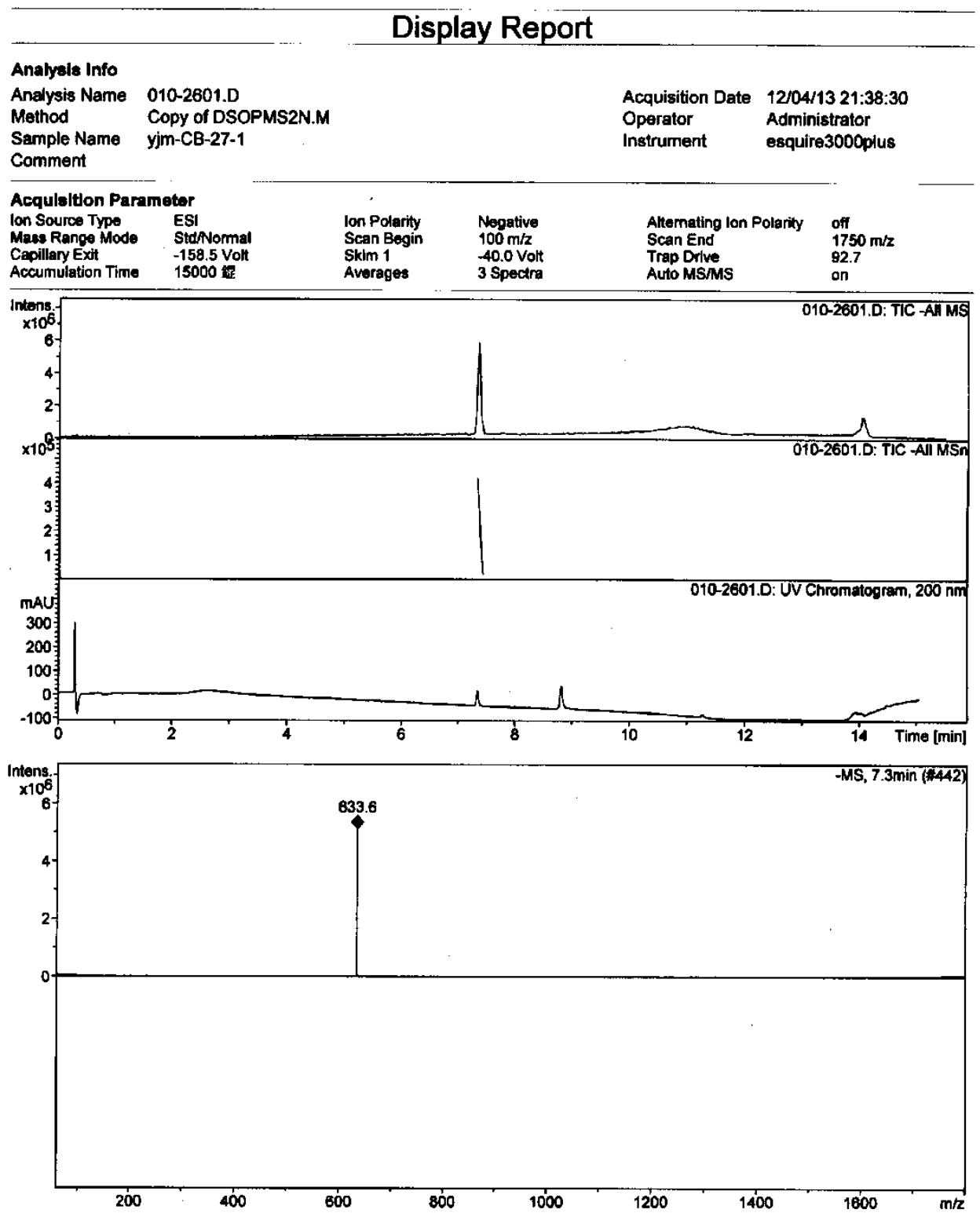


Figure S48. HRESI(+)MS spectrum of 2'-epi-cibacciferin C (6)

Elemental Composition Report

Page 1

\section{Single Mass Analysis}

Tolerance $=5.0 \mathrm{PPM}$ I DBE: $\min =-1.5, \max =50.0$
Element prediction: Off

Number of isotope peaks used for i-FIT $=3$

Monoisotopic Mass, Even Electron lons

249 formula(e) evaluated with 2 results within limits (up to 50 closest results for each mass)

Elements Used:

$\begin{array}{llll}\text { C: } 5-80 & \text { H: } 2-120 & \text { O: } 0-20 & \text { Na: } 0-1\end{array}$

CB-27-1 H. 2-120 0:0-20 NA: $0-1$ LXE KE324

05-Dec-2013

CB-27-1_1205 29 (0.637) AM2 (Ar, 10000.0,0.00,1.00); ABS; Cm (28:48)

1. TOF MSES+

$1.260+004$

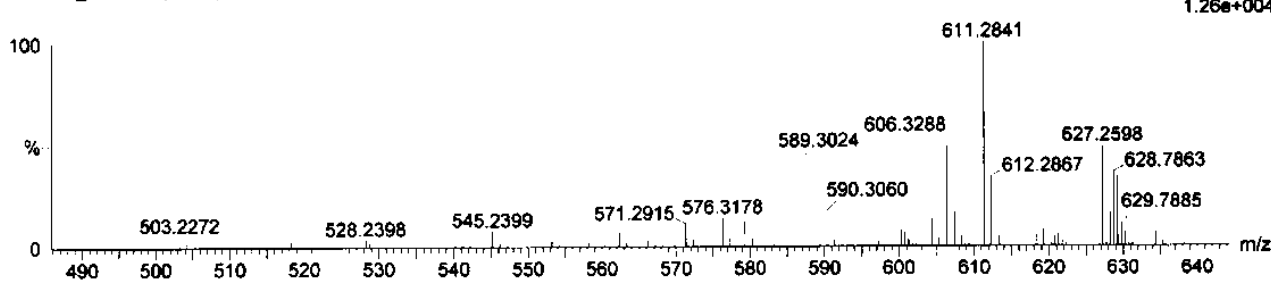

\section{Minimum:}

Maximum:

$\begin{array}{lll}5.0 & 5.0 & -1.5 \\ & 50.0\end{array}$

Mass

Calc. Mass

$\mathrm{mDa}$

PPM

50.0

611.2841

611.2832
611.2856

0.9
-1.5

1.5
-2.5

$\begin{array}{ll}10.5 & 75.0 \\ 13.5 & 77.2\end{array}$

i-EIT

i-FIT (Norm) Formula

0.1
2.2

C32 H44 O10 Na

C34 H43 O10 
Figure S49. IR spectrum of $2^{\prime}$-epi-cibacciferin C (6)

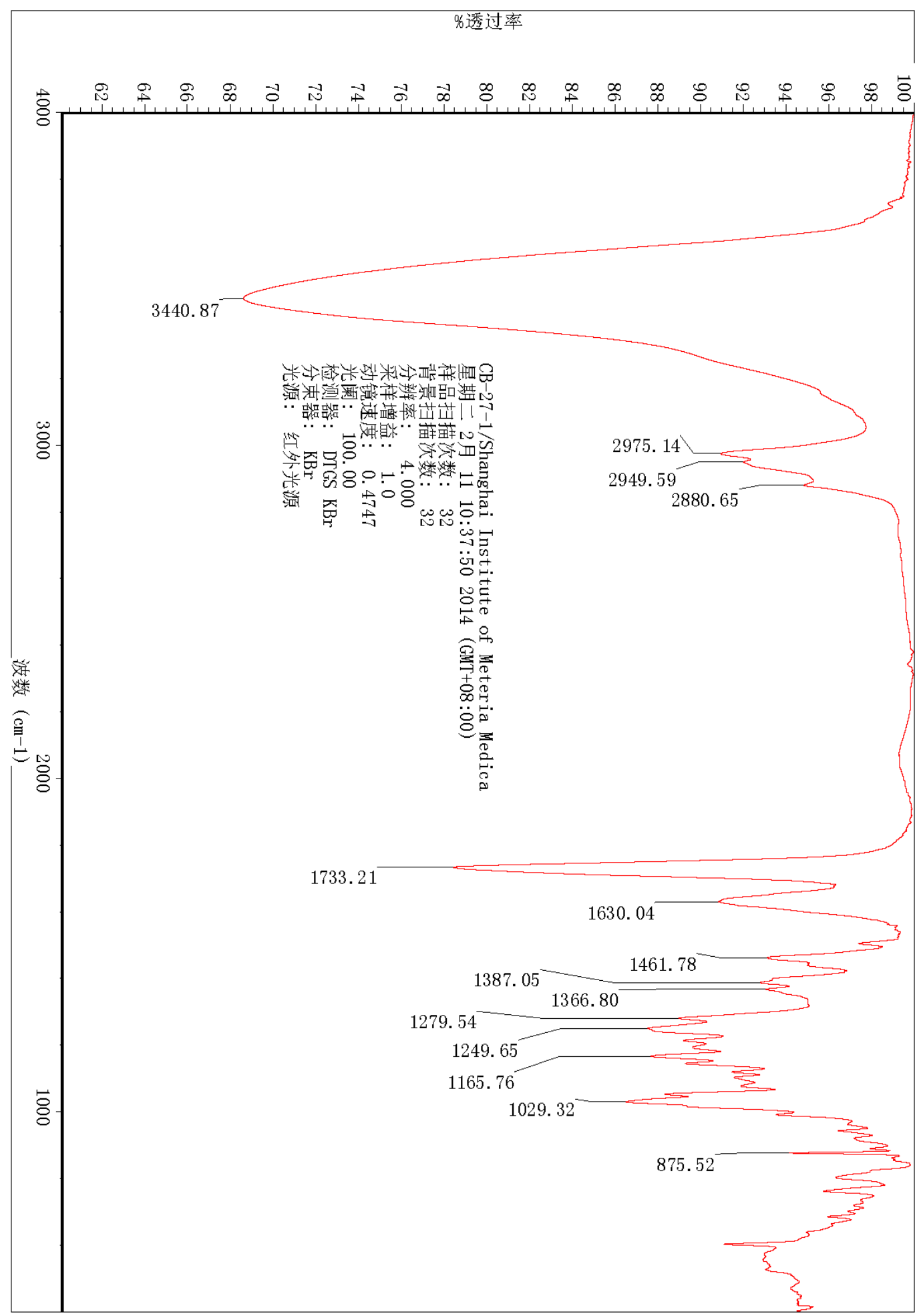


Figure S50. ${ }^{1} \mathrm{H}$ NMR spectrum of $11 \alpha$-acetoxycibacciferin $\mathrm{C}(7)$ in $\mathrm{CDCl}_{3}$

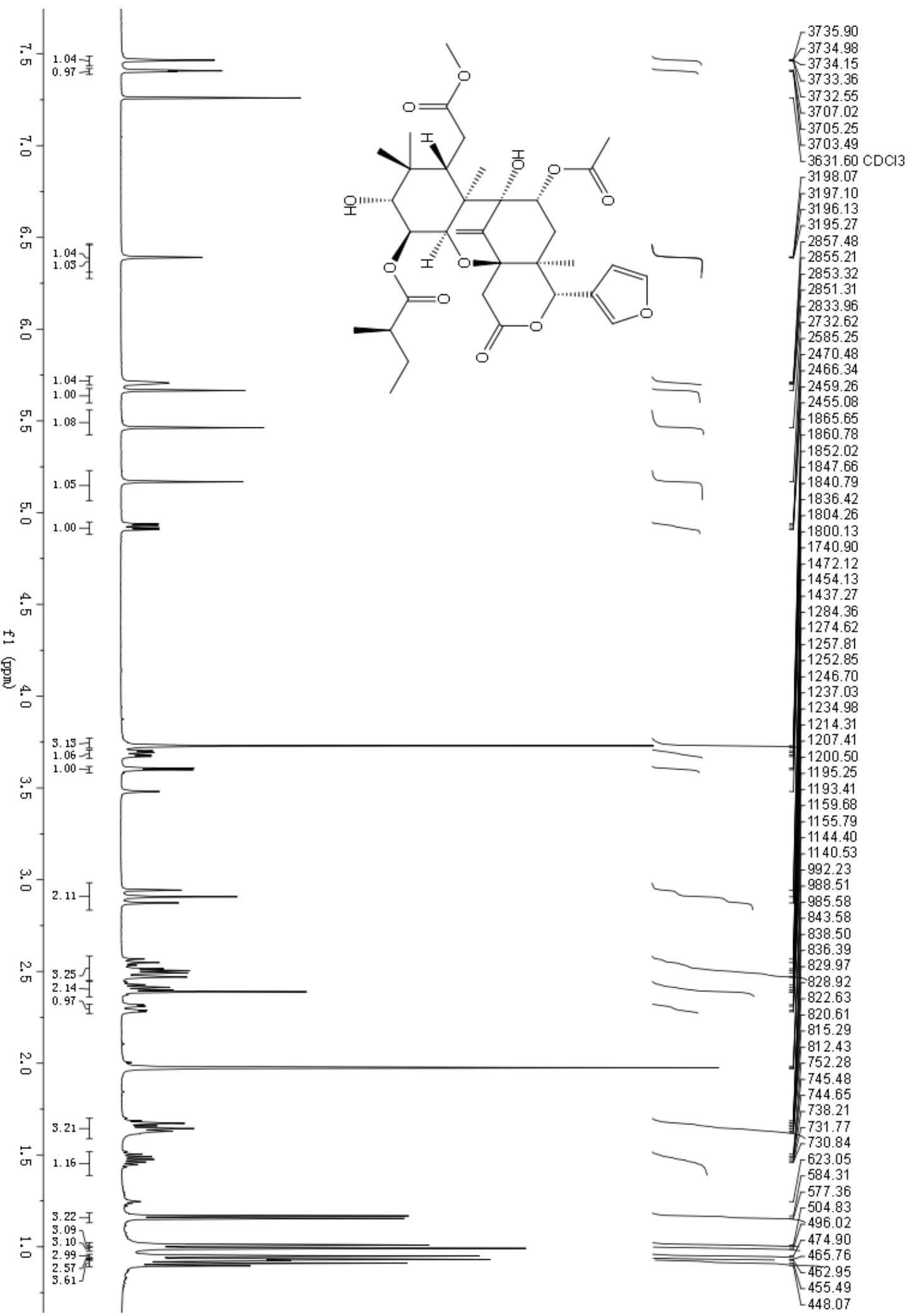


Figure S51. ${ }^{13} \mathrm{C}$ NMR spectrum of $11 \alpha$-acetoxycibacciferin $\mathrm{C}(7)$ in $\mathrm{CDCl}_{3}$

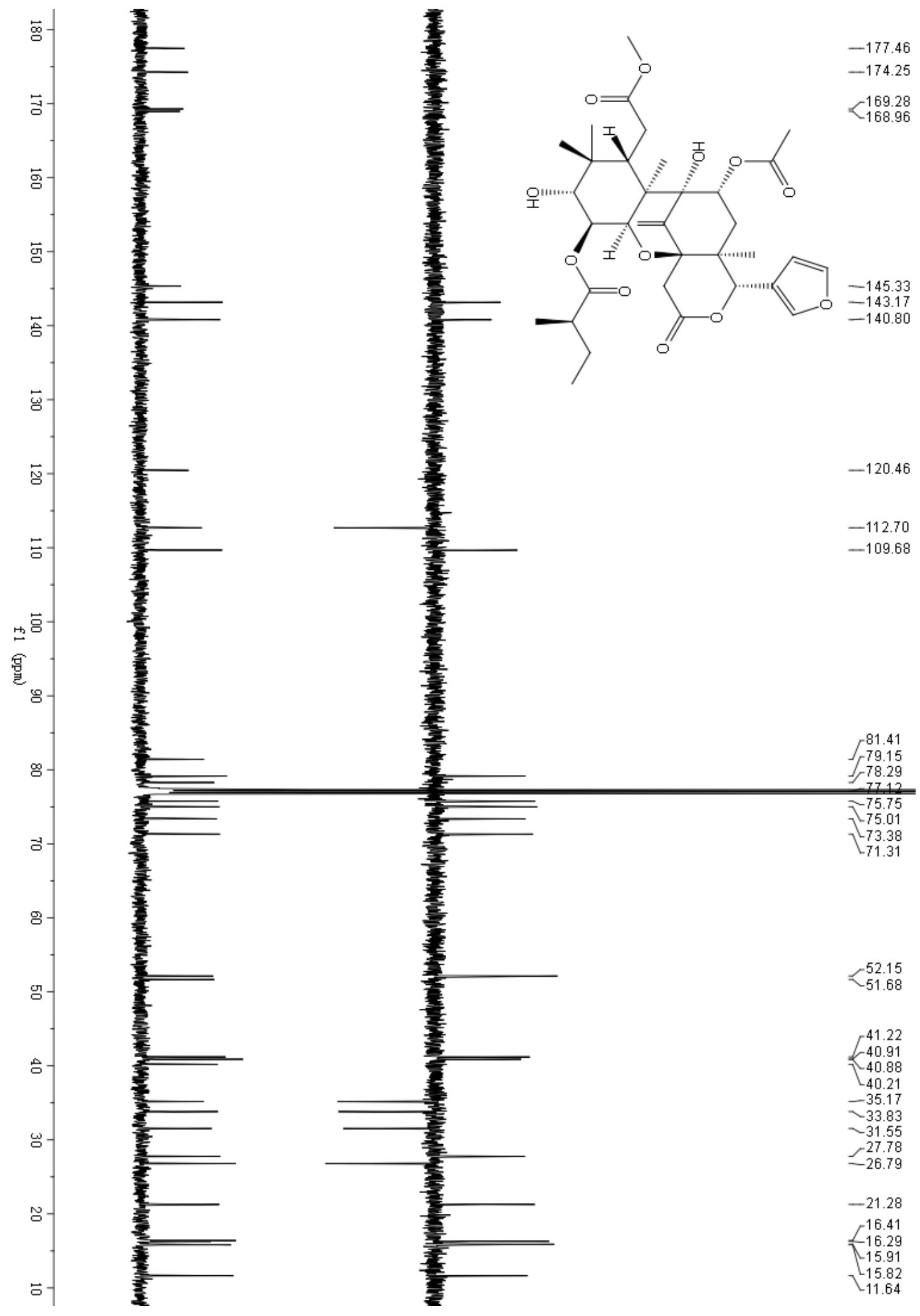


Figure S52. HSQC spectrum of $11 \alpha$-acetoxycibacciferin C (7) in $\mathrm{CDCl}_{3}$

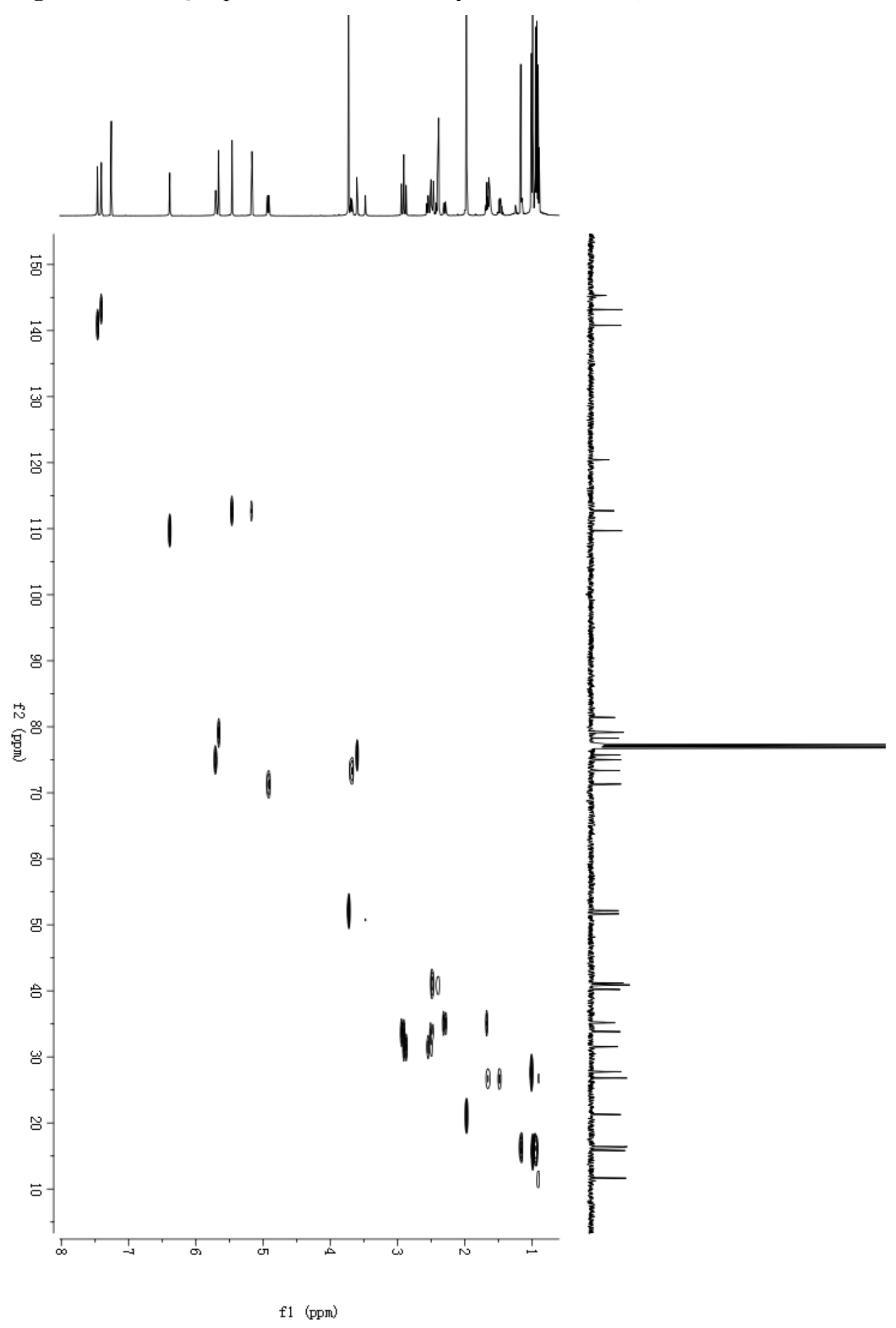

62 
Figure S53. HMBC spectrum of $11 \alpha$-acetoxycibacciferin $\mathrm{C}(7)$ in $\mathrm{CDCl}_{3}$

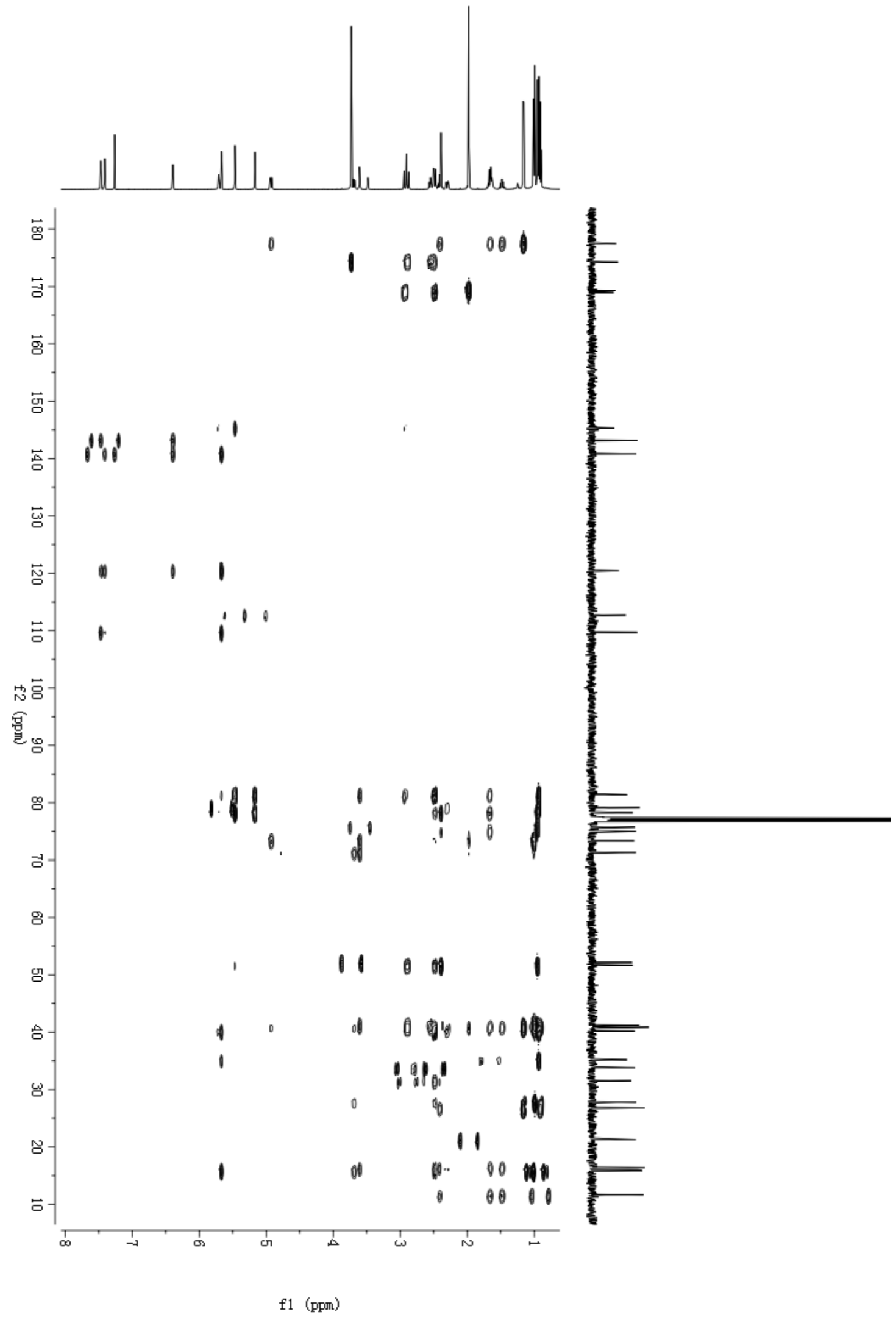


Figure S54. ESI(+)MS spectrum of $11 \alpha$-acetoxycibacciferin C (7)

\section{$\therefore$ कow}
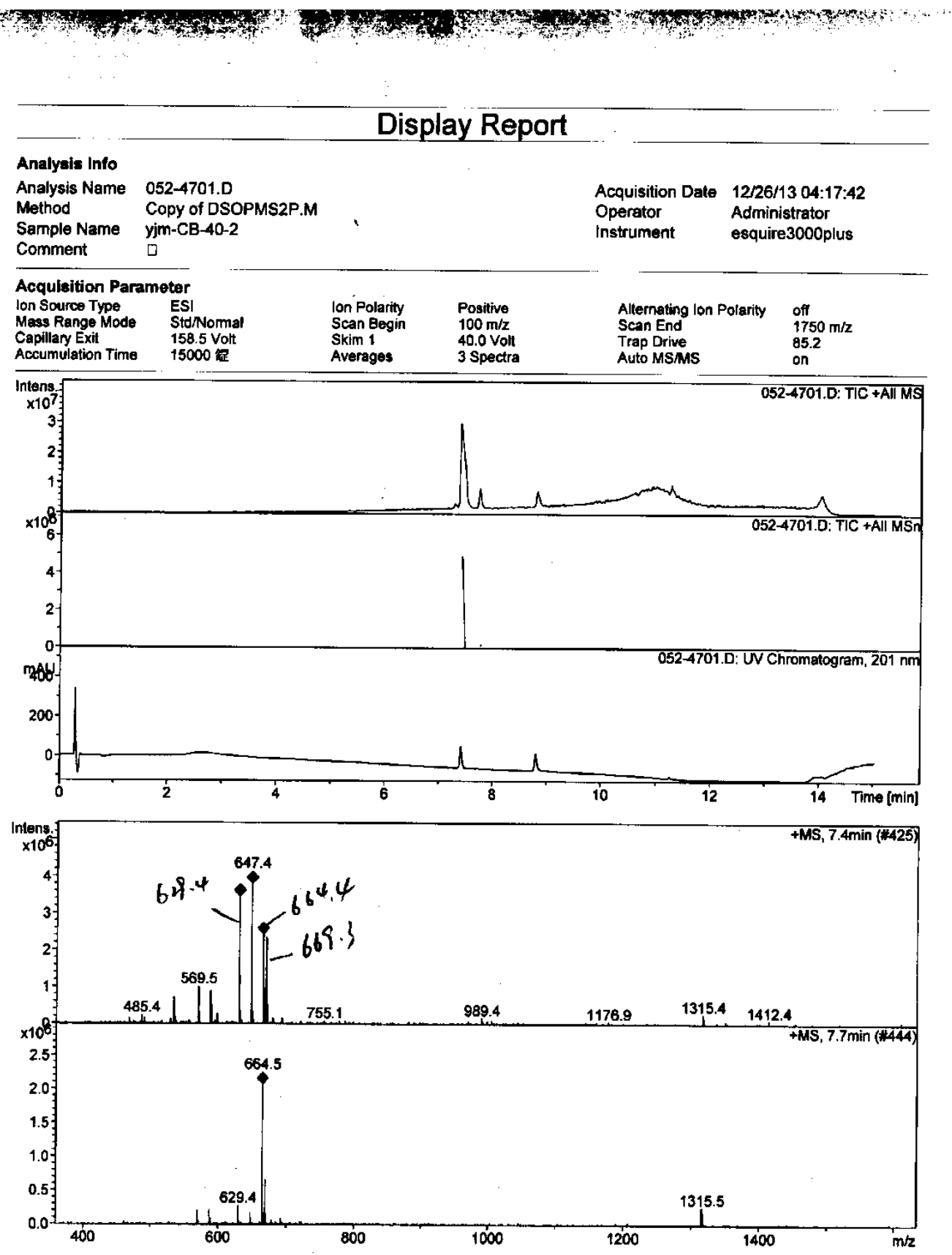
Figure S55. ESI(-)MS spectrum of $11 \alpha$-acetoxycibacciferin C (7)

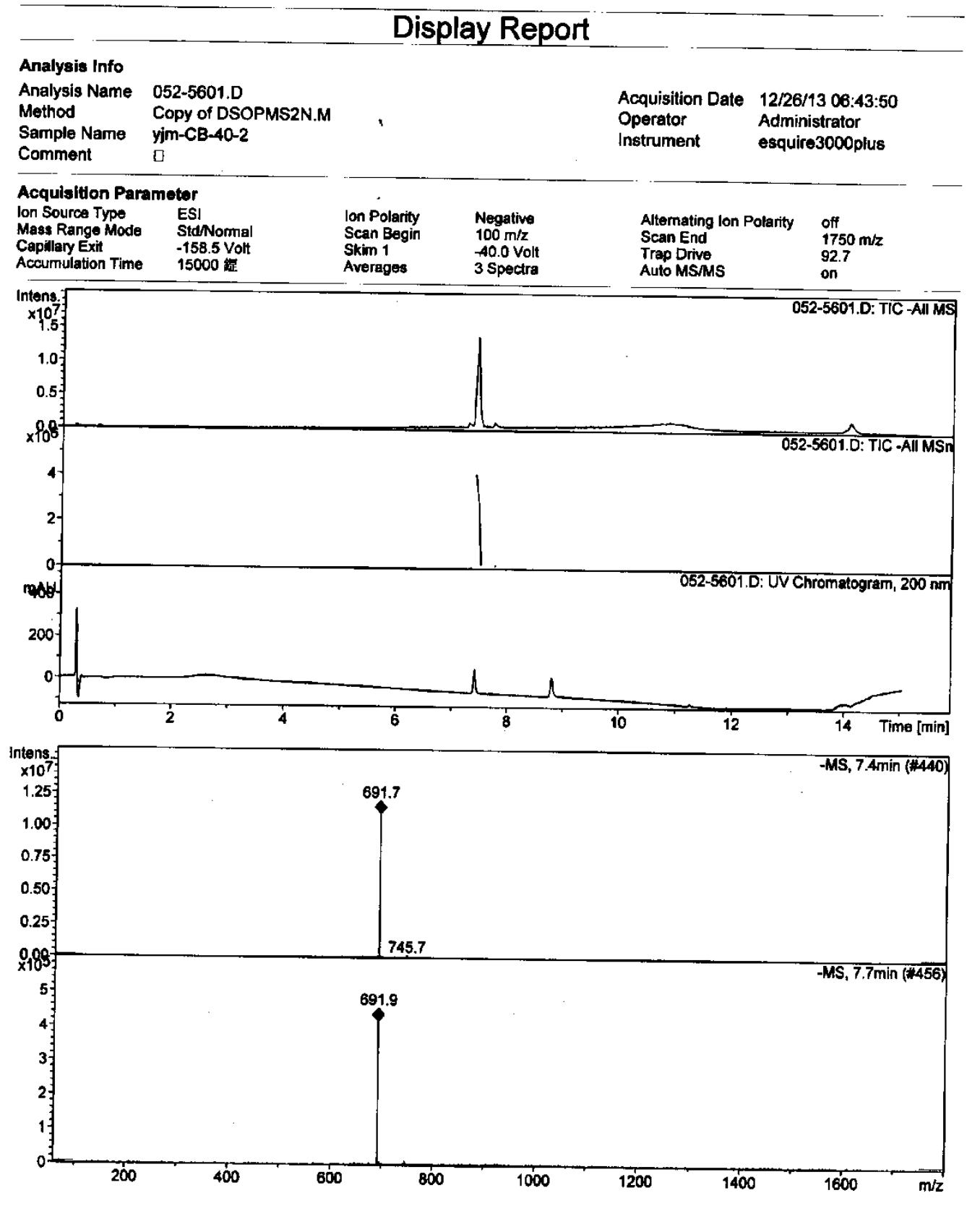


Figure S56. HRESI(+)MS spectrum of $11 \alpha$-acetoxycibacciferin C (7)

Elemental Composition Report

Page 1

Single Mass Analysis

Tolerance $=3.0$ PPM / DBE: $\min =-1.5, \max =50.0$

Element prediction: Off

Number of isotope peaks used for $\mathrm{i}-\mathrm{FIT}=3$

Monoisotopic Mass, Even Electron lons

278 formula(e) evaluated with 2 results within limits' (up to 50 closest results for each mass)

$\begin{array}{llll}\text { C: } 5-80 & \text { H: } 2-120 & \text { O: } 0-20 \quad \mathrm{Na}: 0-1\end{array}$

CB-40-2 . LCT PXE KE324

C8-40-2_20140403 33 (0.725) AM2 (Ar, 10000.0,0.00,1.00); ABS; Cm (23:39)

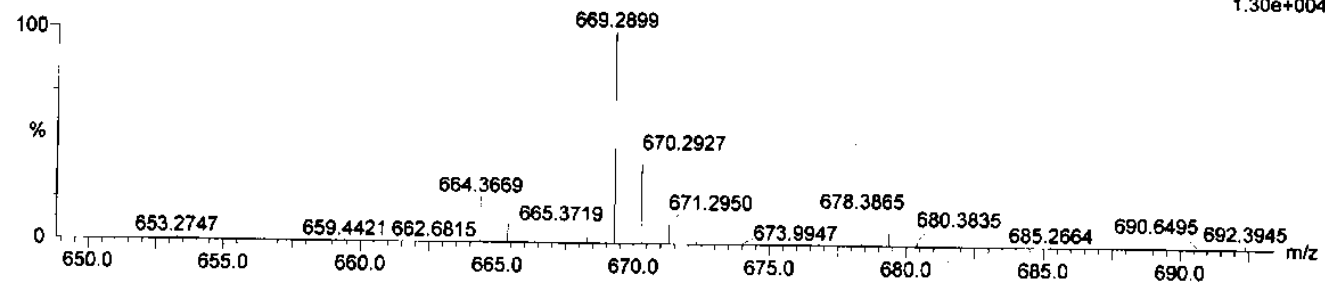

Minimum:

Maxirnum:

$\begin{array}{lll}5.0 & 3.0 & -1.5 \\ & 50.0\end{array}$

Mass Calc. Mass MDa PPM DBE

$669.2899 \quad 669.2911 \quad-1.2 \quad-1.8 \quad 14.5$

i-FIT i-EIT (Norm) Formula

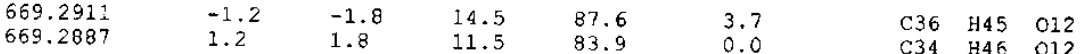

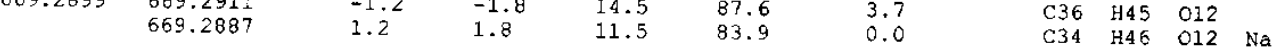


Figure S57. IR spectrum of $11 \alpha$-acetoxycibacciferin C (7)

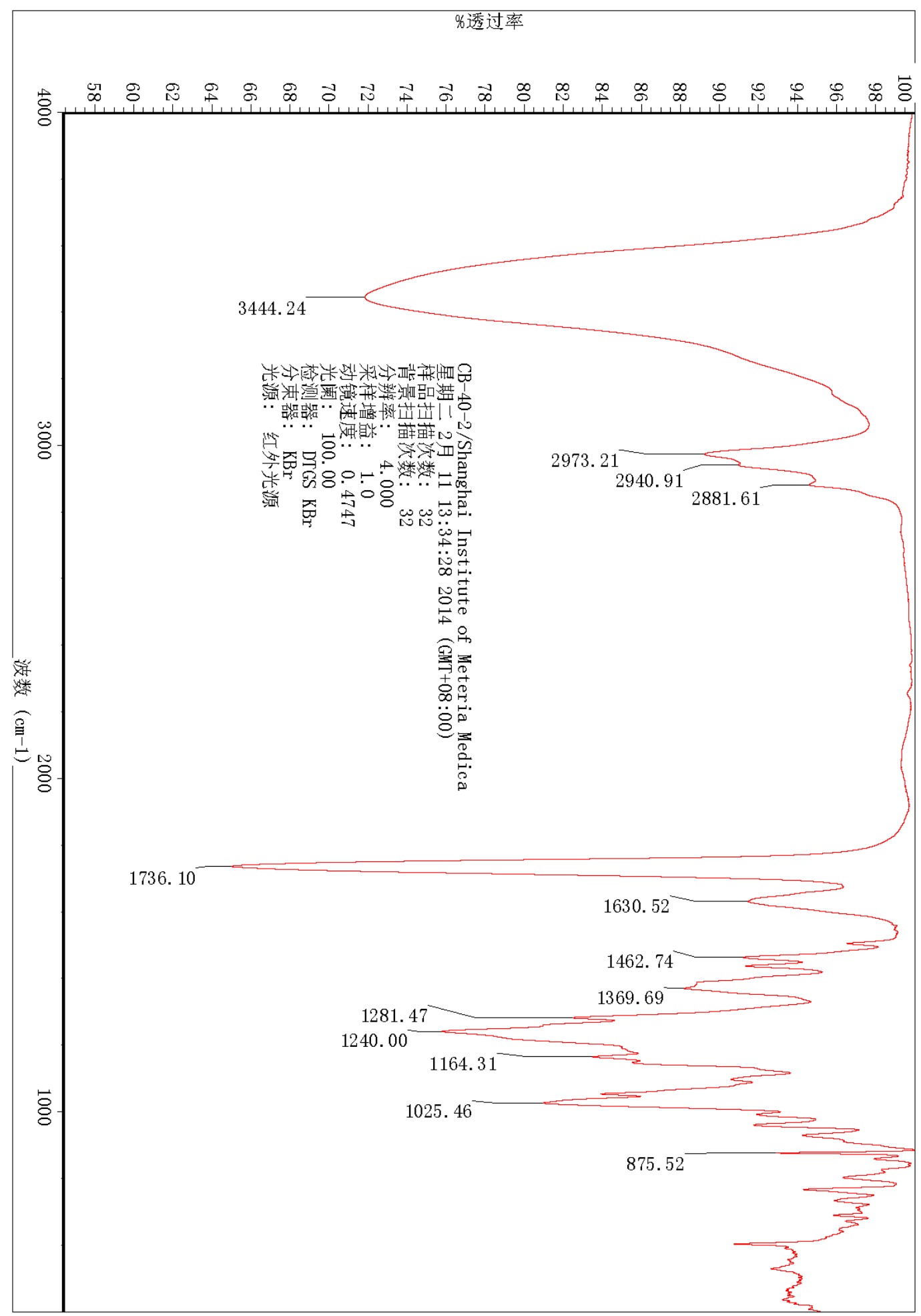


Figure S58. ${ }^{1} \mathrm{H}$ NMR spectrum of cibacciferin $\mathrm{D}(\mathbf{8})$ in $\mathrm{CDCl}_{3}$
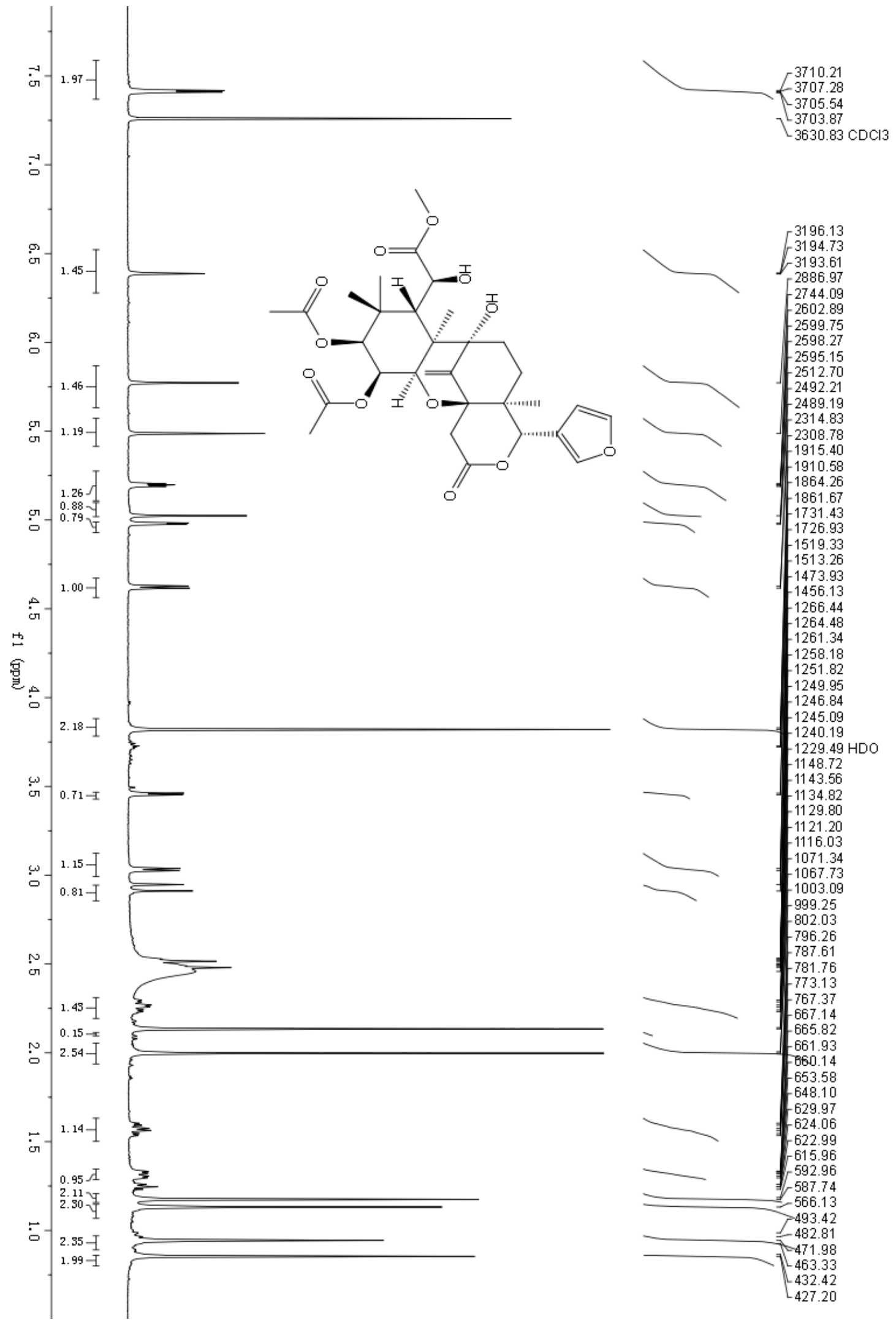
Figure S59. ${ }^{13} \mathrm{C}$ NMR spectrum of cibacciferin $\mathrm{D}(\mathbf{8})$ in $\mathrm{CDCl}_{3}$

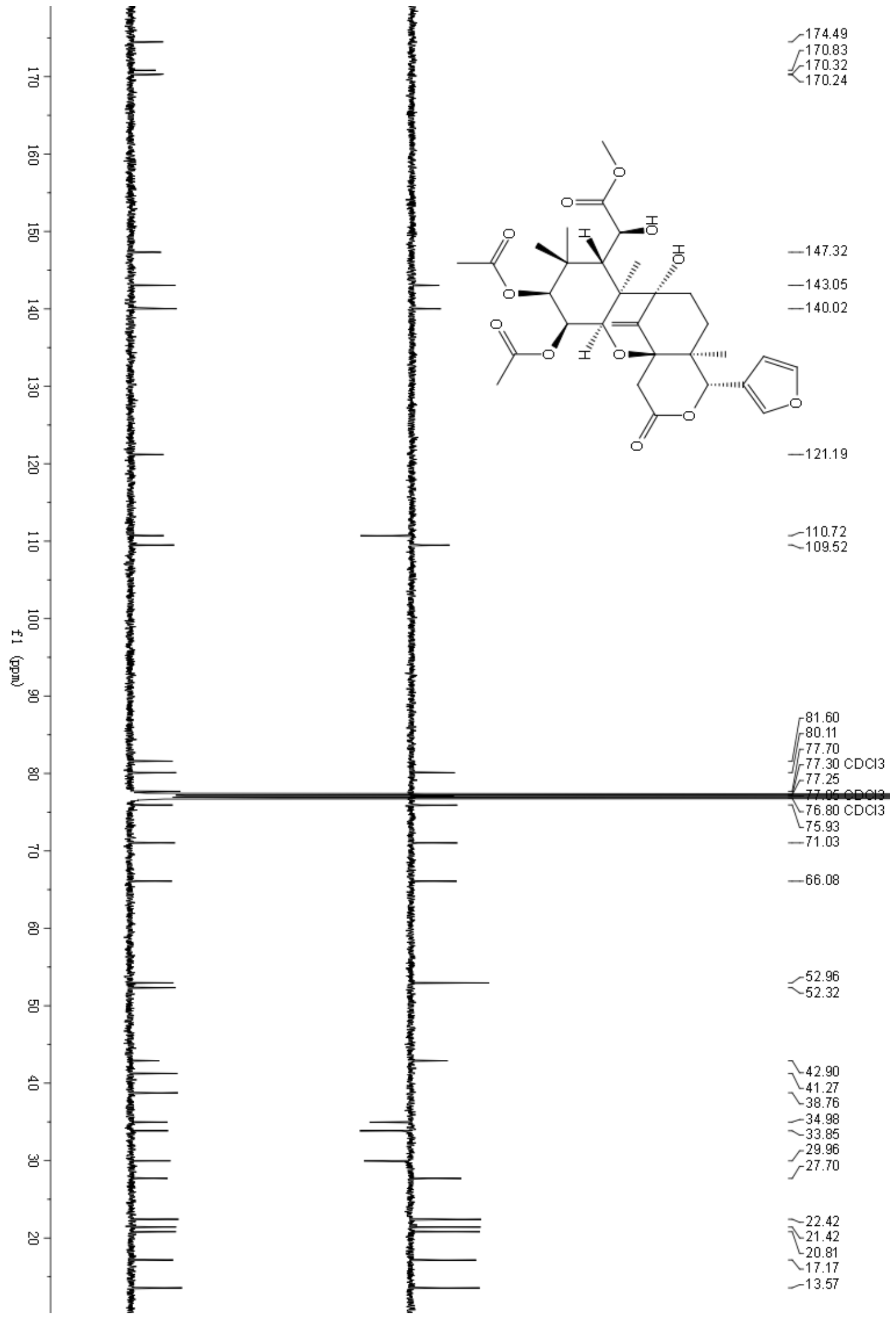


Figure S60. HSQC spectrum of cibacciferin D (8) in $\mathrm{CDCl}_{3}$

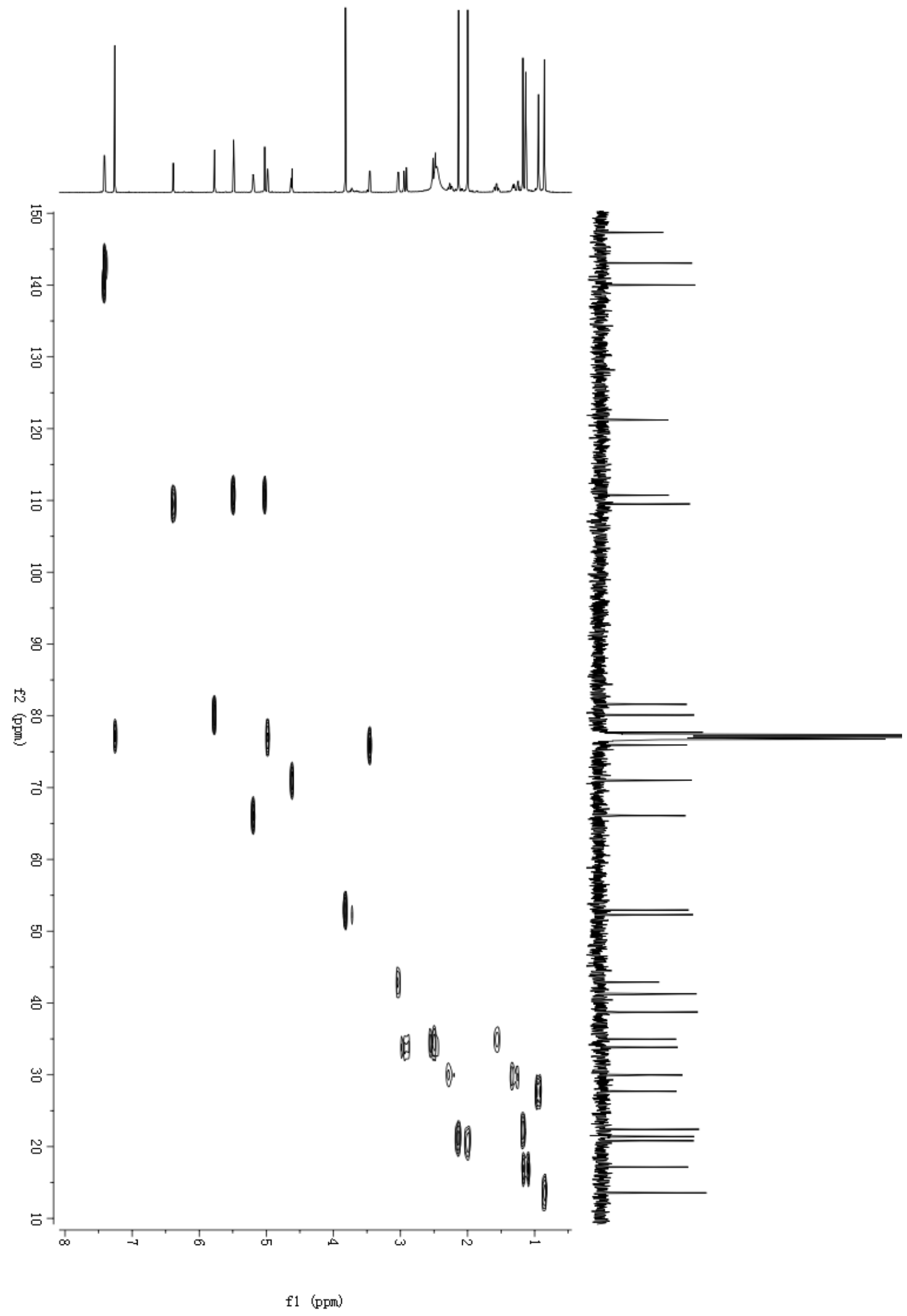


Figure S61. HMBC spectrum of cibacciferin D (8) in $\mathrm{CDCl}_{3}$

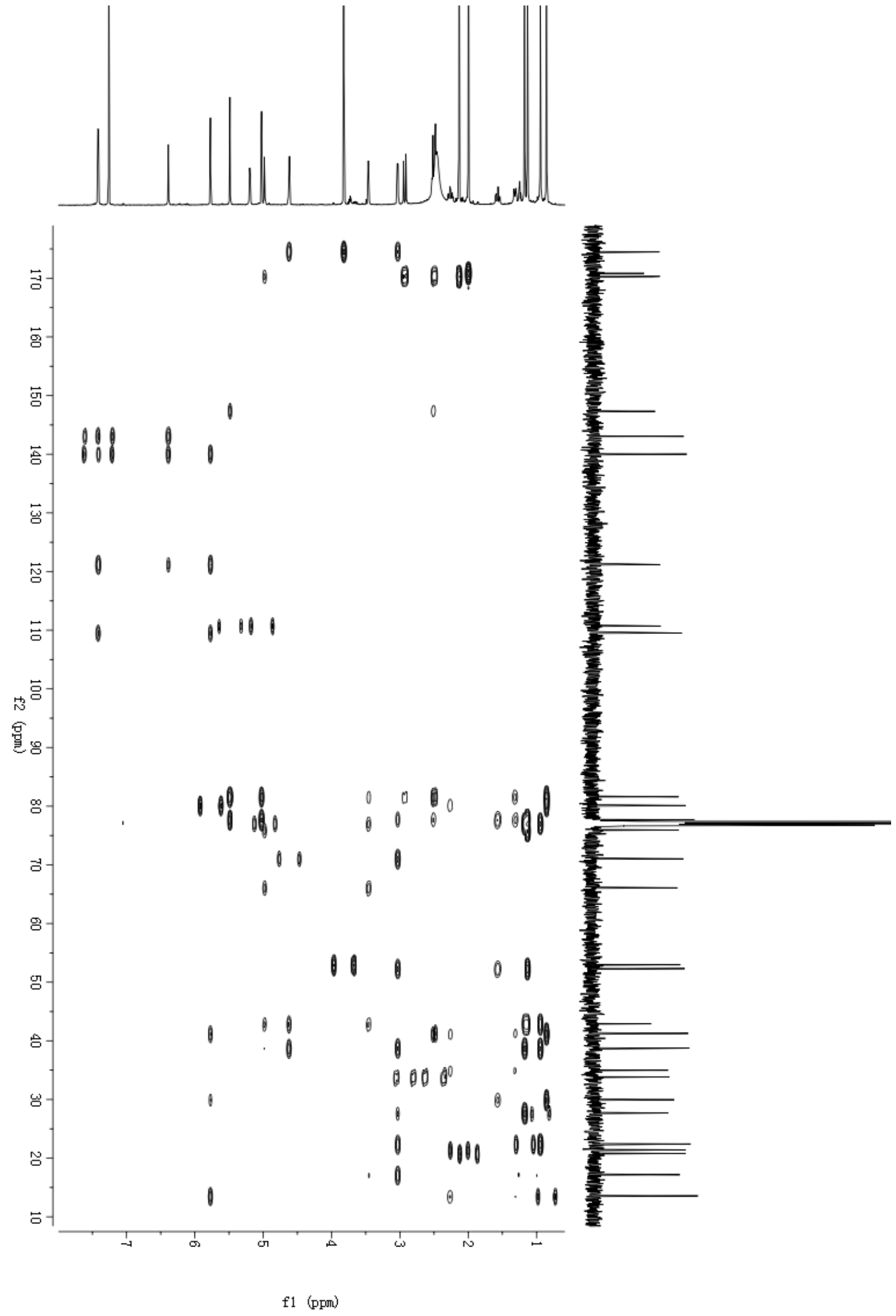


Figure S62. ROESY spectrum of cibacciferin D (8) in $\mathrm{CDCl}_{3}$

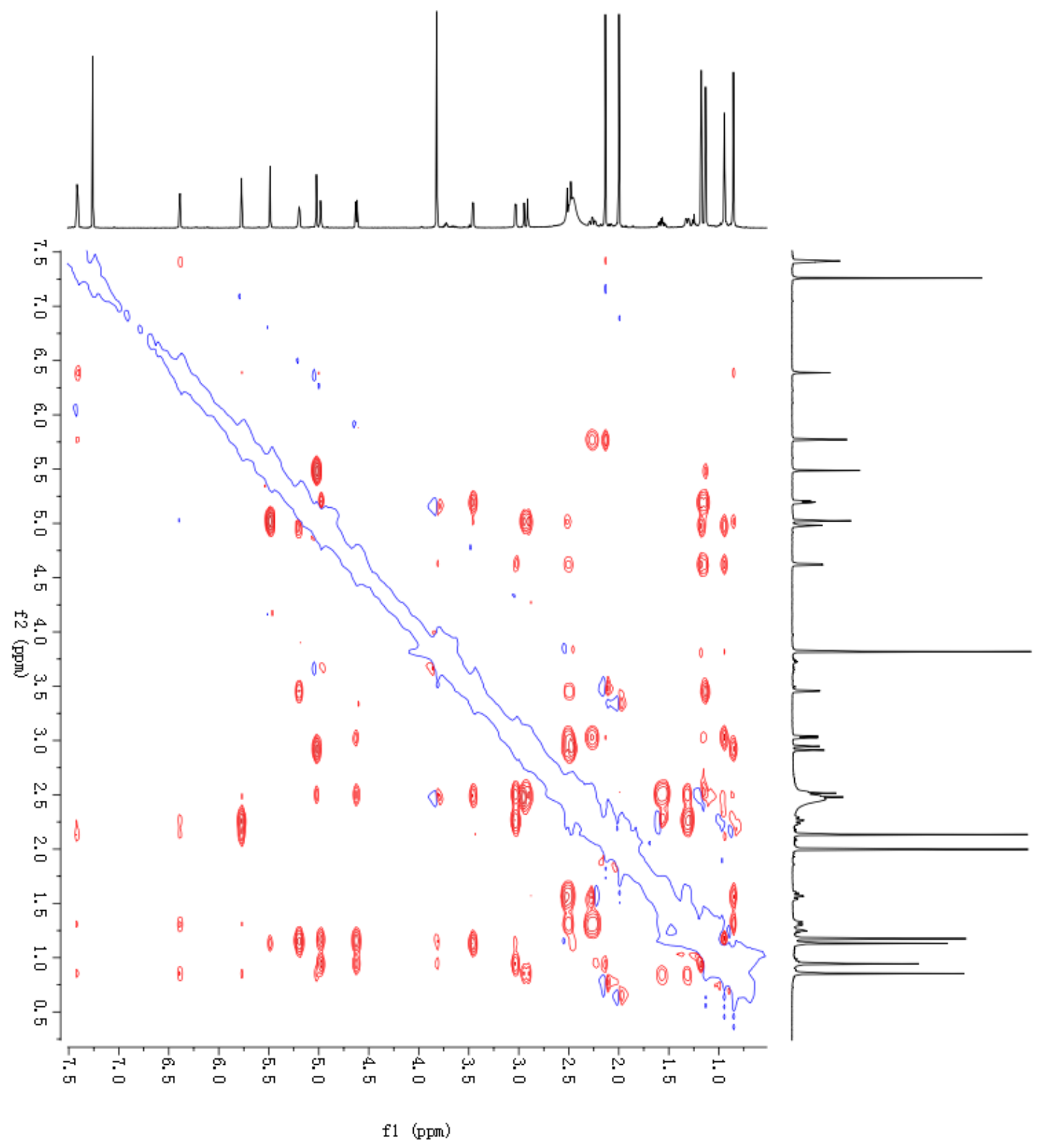


Figure S63. ESI(+)MS spectrum of cibacciferin D (8)

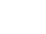

Analysis Info

$\begin{array}{ll}\text { Analysis Name } & 002-0501 . D \\ \text { Method } & \text { Copy of DSOPMS2P.M } \\ \text { Sample Name } & \text { yjm-cc-57 }\end{array}$

Comment

Acquisition Parameter

Ion Source Type ESI

Mass Range Mode Std/Normal

Accumulation Time

$158.5 \mathrm{Volt}$

Display Report
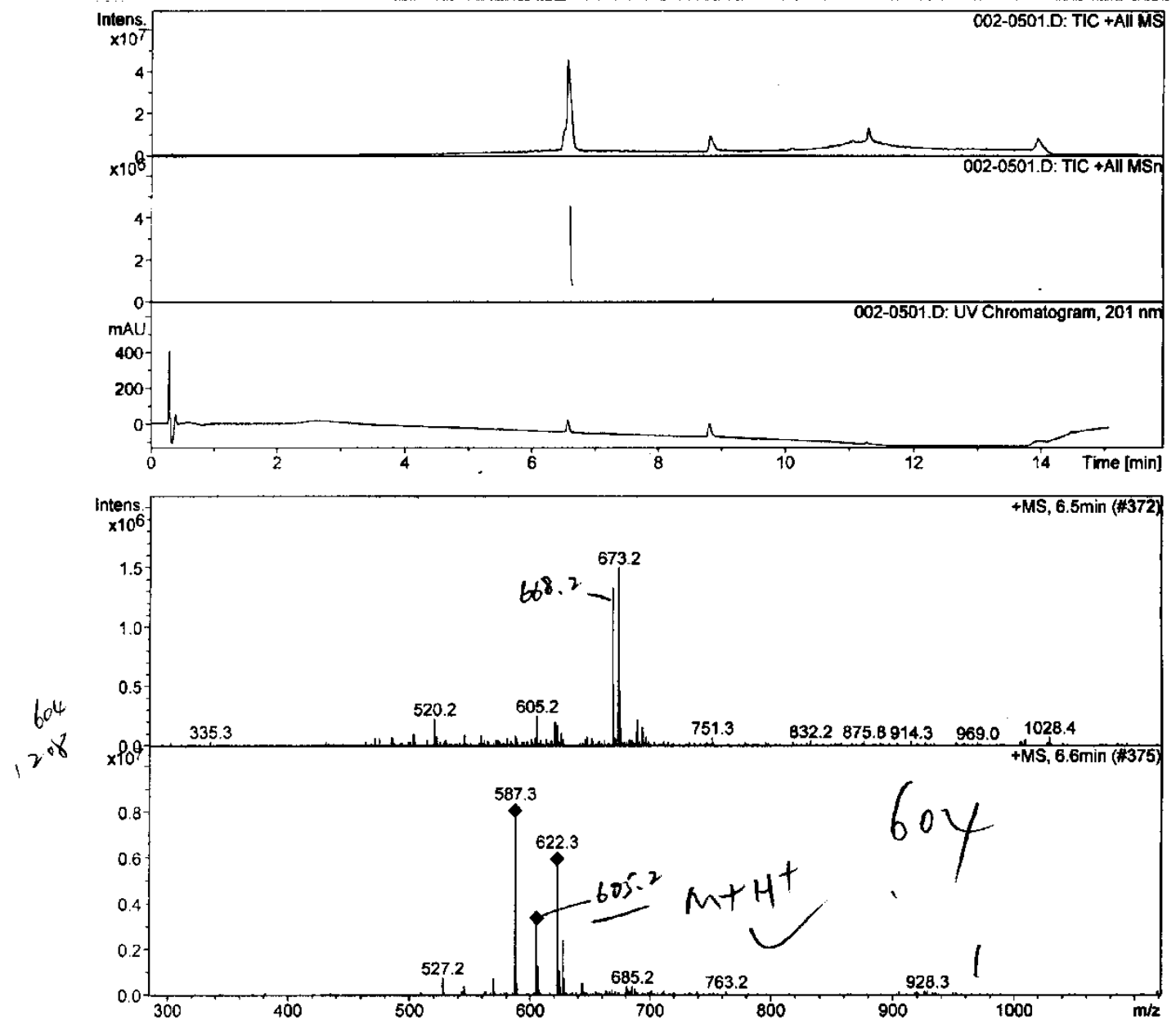

Bruker Daltonics DataAnalysis 3.1

printed:

$05 / 29 / 13$

$09: 32: 21$

Page 1 of 1 
Figure S64. ESI(-)MS spectrum of cibacciferin D (8)

\section{Display Report}

Analysis Info

Analysis Name 002-2001.D

Method Capy of DSOPMS2N.M

Acquisition Date 05/28/13 21:29:03

Sample Name yjm-cc-57

Operator Administrator

Comment

Acquisition Parameter

$\begin{array}{llll}\text { Ion Source Type } & \text { ESI } & \text { Ion Polarity } & \text { Negative } \\ \text { Mass Range Mode } & \text { Std/Normal } & \text { Scan Begin } & 100 \mathrm{~m} / \mathrm{z}\end{array}$

\begin{tabular}{|c|c|c|c|c|c|}
\hline $\begin{array}{l}\text { Capillary Exit } \\
\text { Accumulation Time }\end{array}$ & $\begin{array}{l}\text { Std/Normal } \\
-158.5 \text { Volt } \\
15000 \text { 埕: }\end{array}$ & $\begin{array}{l}\text { Scan Begin } \\
\text { Skim } 1 \\
\text { Averages }\end{array}$ & $\begin{array}{l}100 \mathrm{~m} / \mathrm{z} \\
-40.0 \text { Volt } \\
3 \text { Spectra }\end{array}$ & $\begin{array}{l}\text { Scan End } \\
\text { Trap Drive } \\
\text { Auto MS/MS }\end{array}$ & $\begin{array}{l}1750 \mathrm{~m} / 2 \\
92.7 \\
\text { on }\end{array}$ \\
\hline
\end{tabular}

Averages 3 Spectra

Instrument

esquire 3000 plus
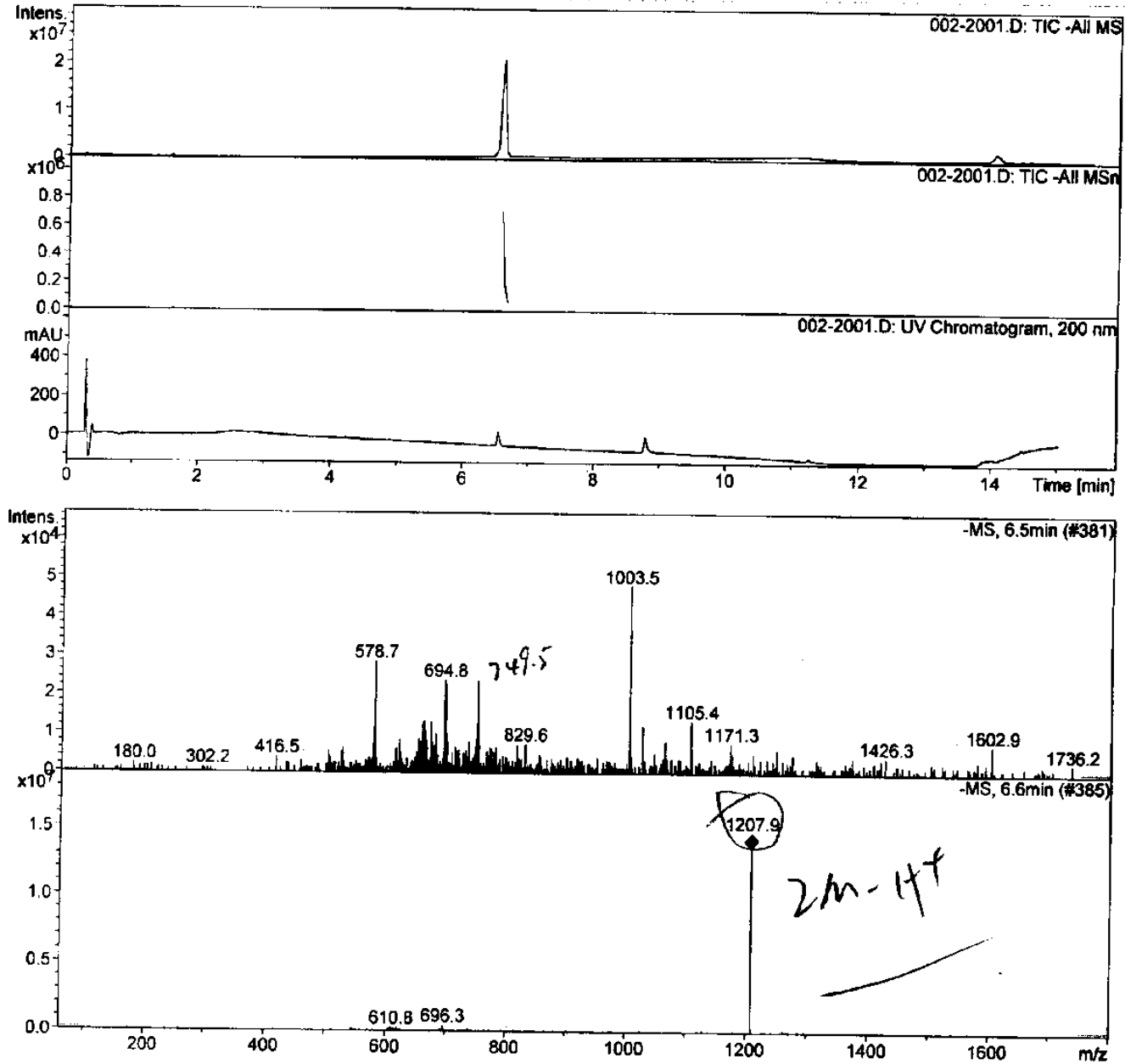
Figure S65. HRESI(+)MS spectrum of cibacciferin D (8)

\section{Elemental Composition Report}

\section{Singie Mass Analysis}

Tolerance $=3.0$ PPM / DBE: $\min =-1.5, \max =50.0$

Element prediction: Off

Number of isotope peaks used for i-FIT $=3$

Monoisotopic Mass, Even Electron lons

257 formula(e) evaluated with 1 results within limits (up to 50 closest results for each mass)

Elements Used:

$\begin{array}{llll}\text { C: } 6-80 & \text { H: } 2-120 & \text { O: } 0-20 & \mathrm{Na}: 0-1\end{array}$

CC-57

LCT PXE KE324

CC-57_0608 29 (0.636) AM2 (Ar, 10000.0,0.00,1.00); ABS; Cm (19:38)

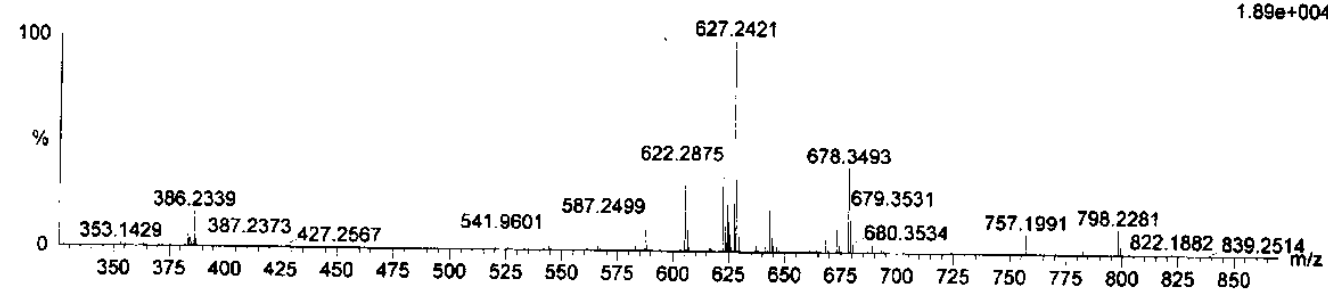
Minimum:

Maximum:

$\begin{array}{lll}3.0 & 3.0 & -1.5 \\ & 50.0\end{array}$

Mass Calc. Mass mDa PPM

$\begin{array}{llllllllllll}627.2421 & 627.2417 & 0.4 & 0.6 & 11.5 & 81.4 & 0.0 & \mathrm{C} 31 & \mathrm{H} 40 & 012 \mathrm{Na}\end{array}$

DBE i-FIT i-EIT (Norm) Formula 
Figure S66. IR spectrum of cibacciferin D (8)

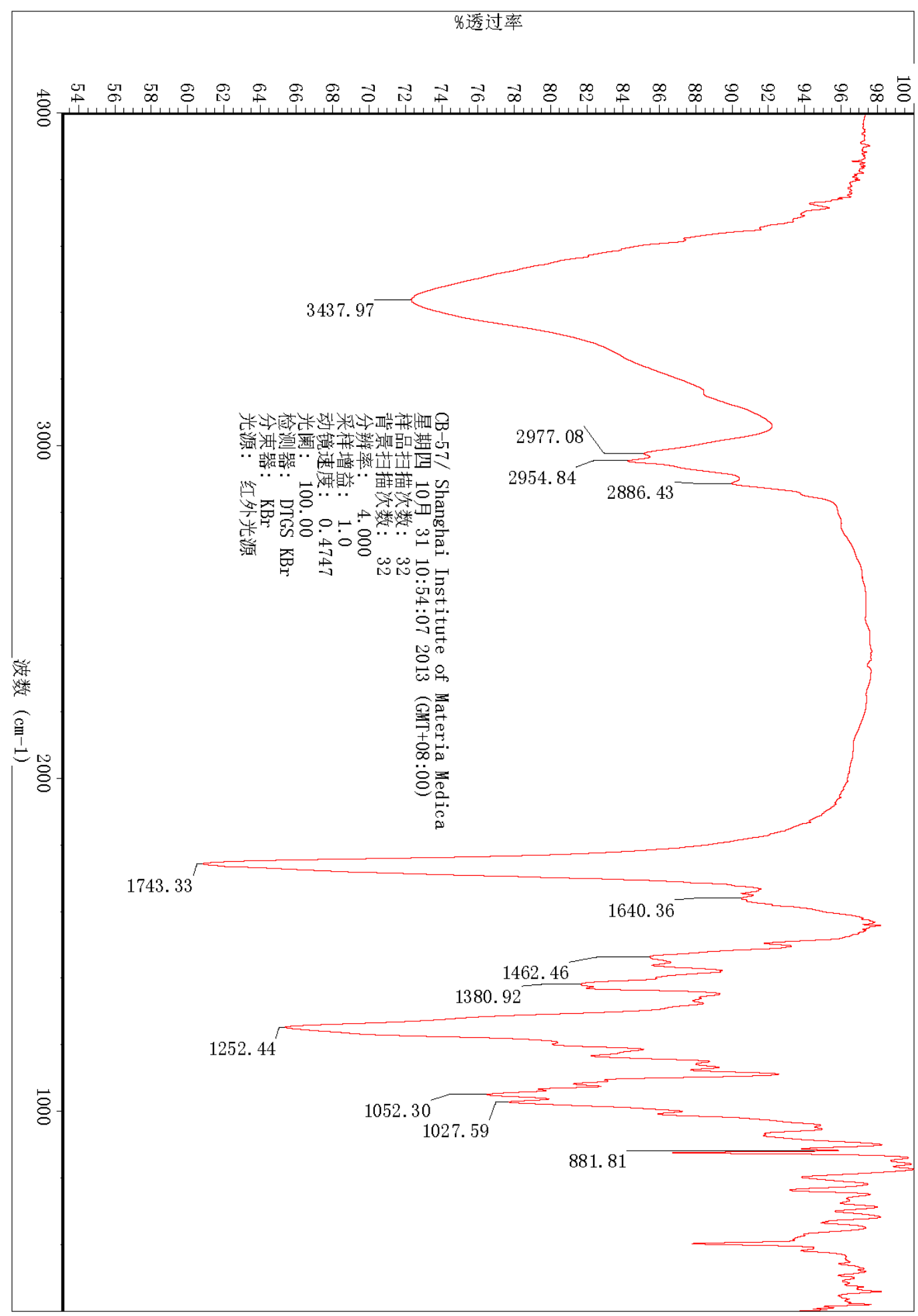


Figure S67. ${ }^{1} \mathrm{H}$ NMR spectrum of cibacciferin $\mathrm{E}(\mathbf{9})$ in $\mathrm{CDCl}_{3}$

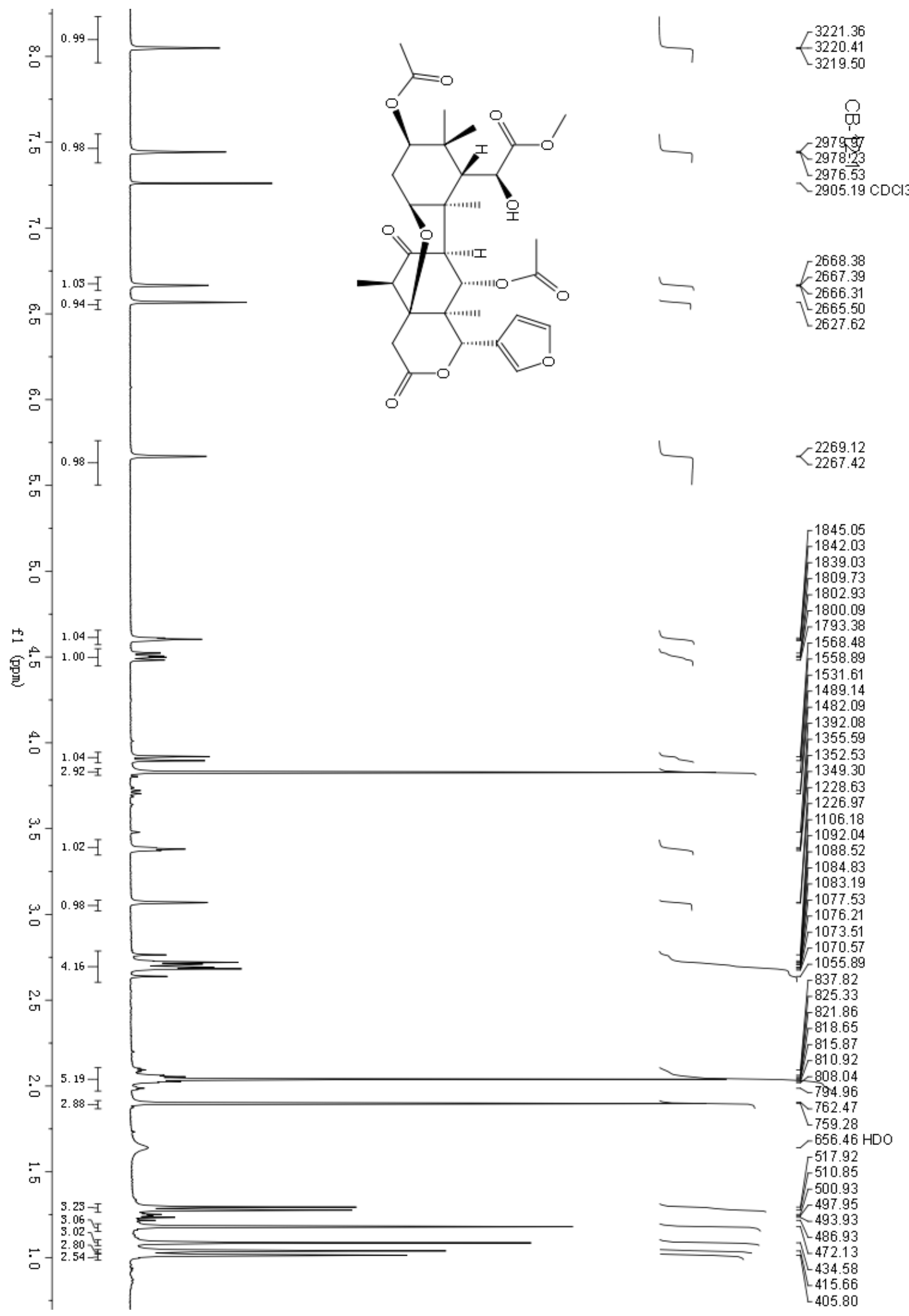


Figure S68. ${ }^{13} \mathrm{C}$ NMR spectrum of cibacciferin $\mathrm{E}(\mathbf{9})$ in $\mathrm{CDCl}_{3}$

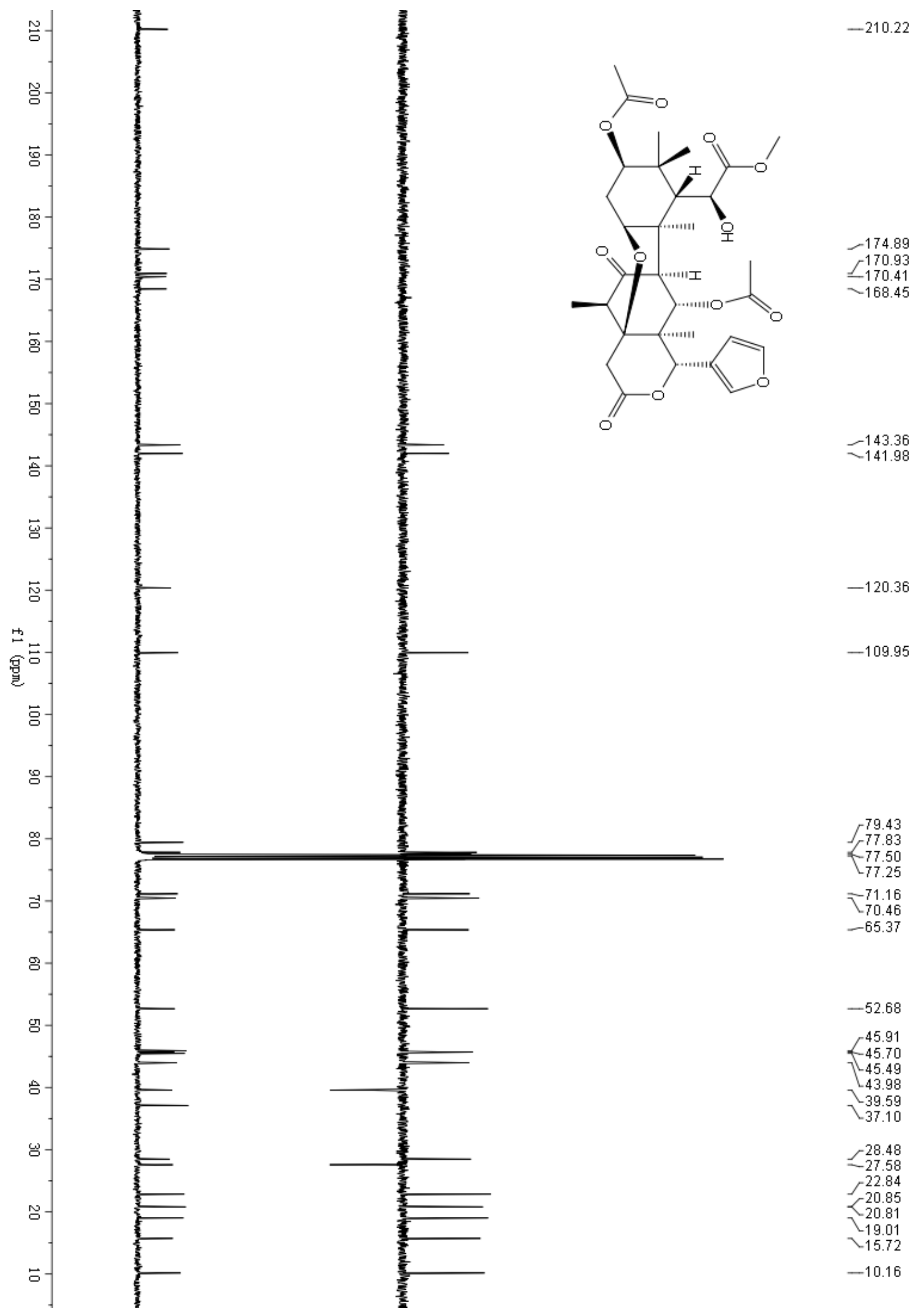


Figure S69. HSQC spectrum of cibacciferin $\mathrm{E}(\mathbf{9})$ in $\mathrm{CDCl}_{3}$

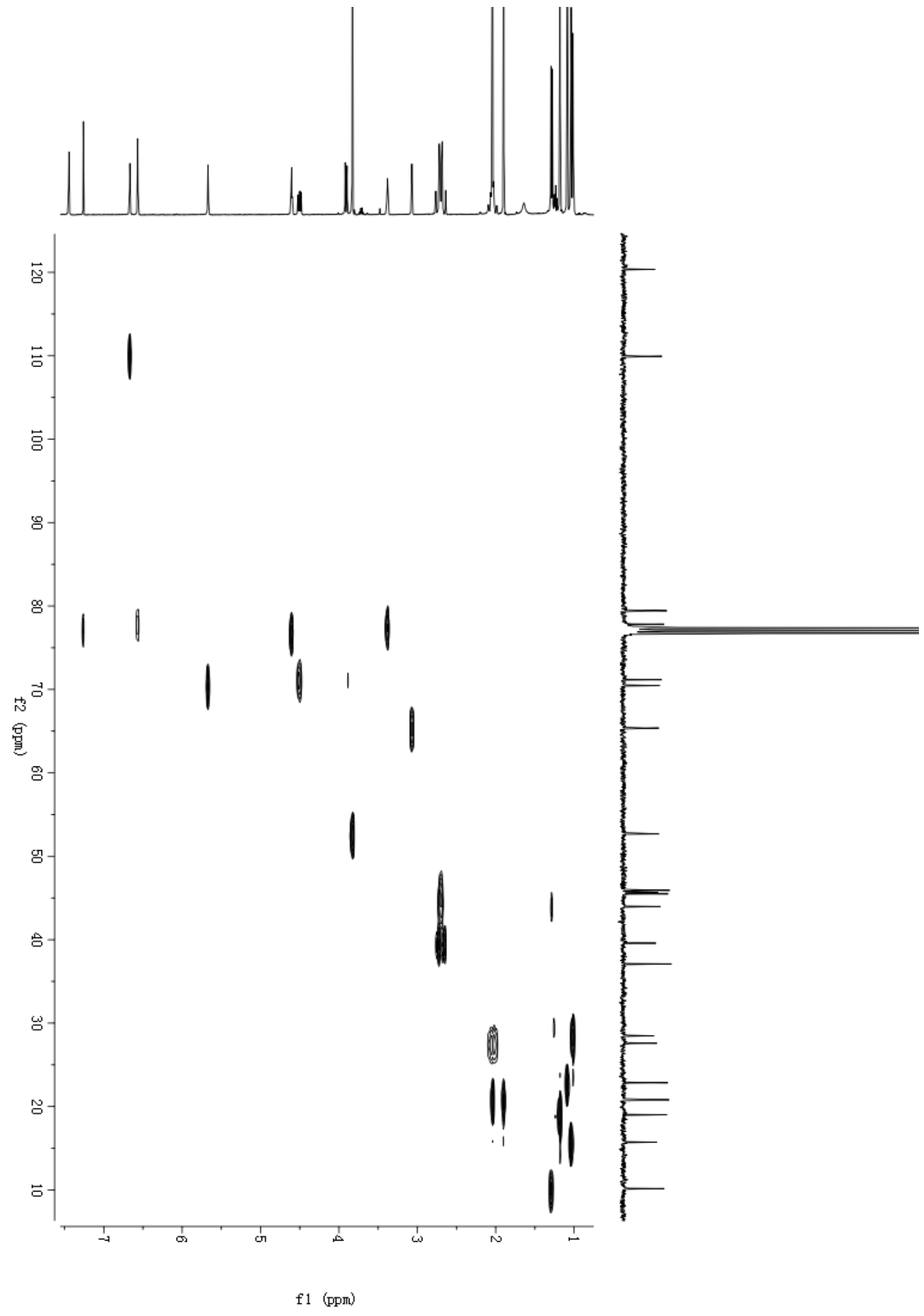


Figure S70. $\mathrm{HMBC}$ spectrum of cibacciferin $\mathrm{E}(\mathbf{9})$ in $\mathrm{CDCl}_{3}$

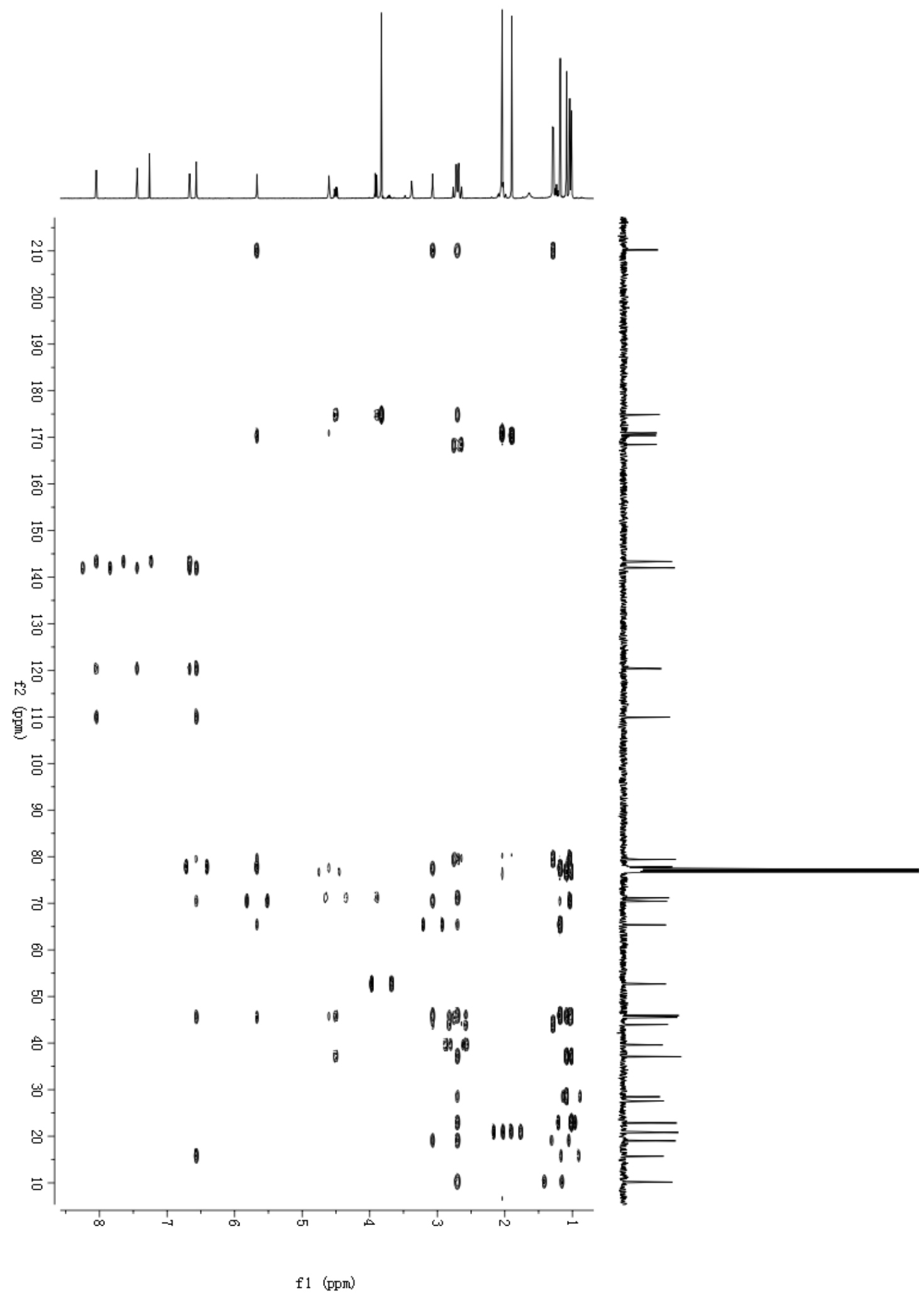


Figure S71. ROESY spectrum of cibacciferin E (9) in $\mathrm{CDCl}_{3}$

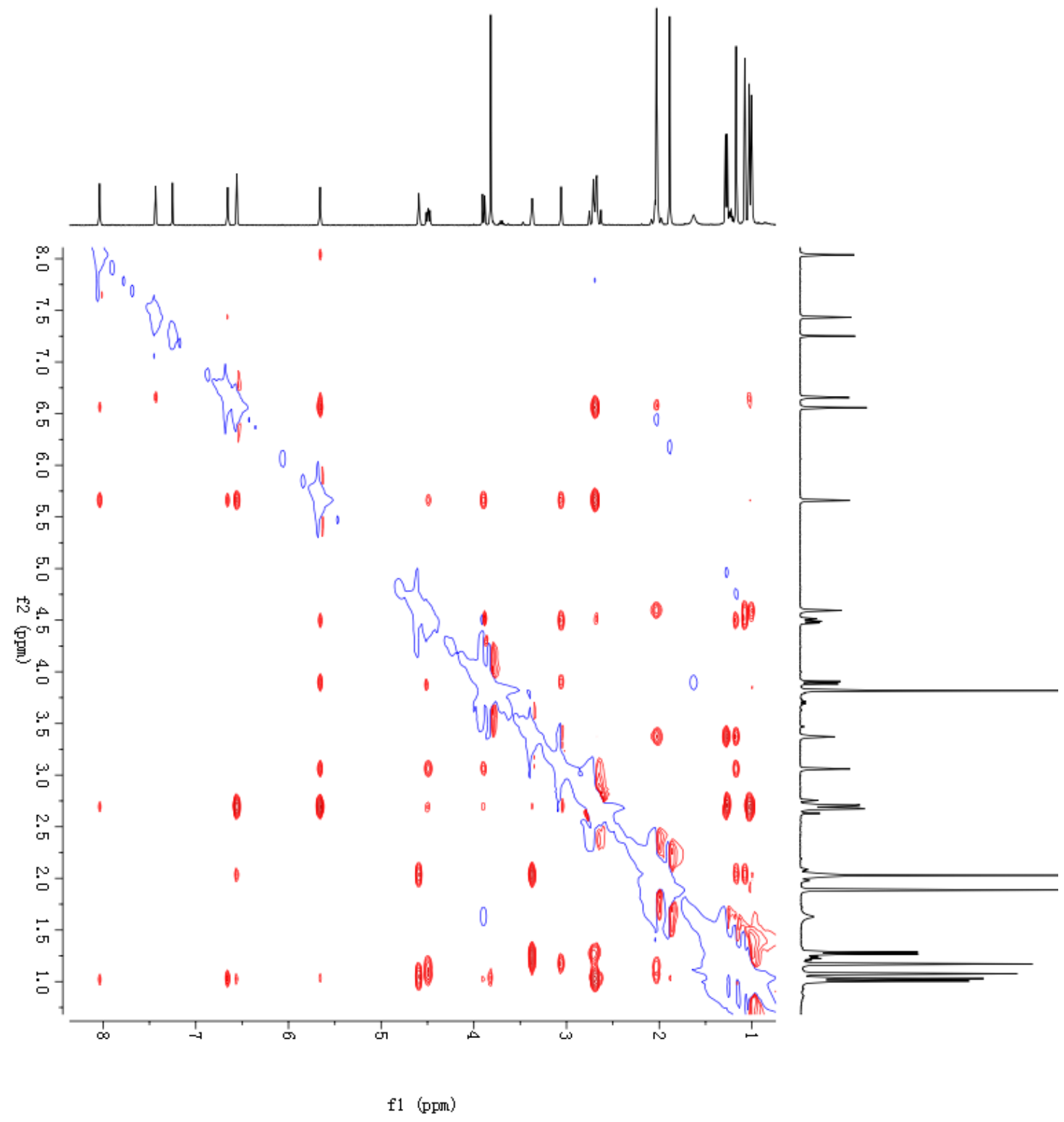


Figure S72. ESI(+)MS spectrum of cibacciferin E (9)

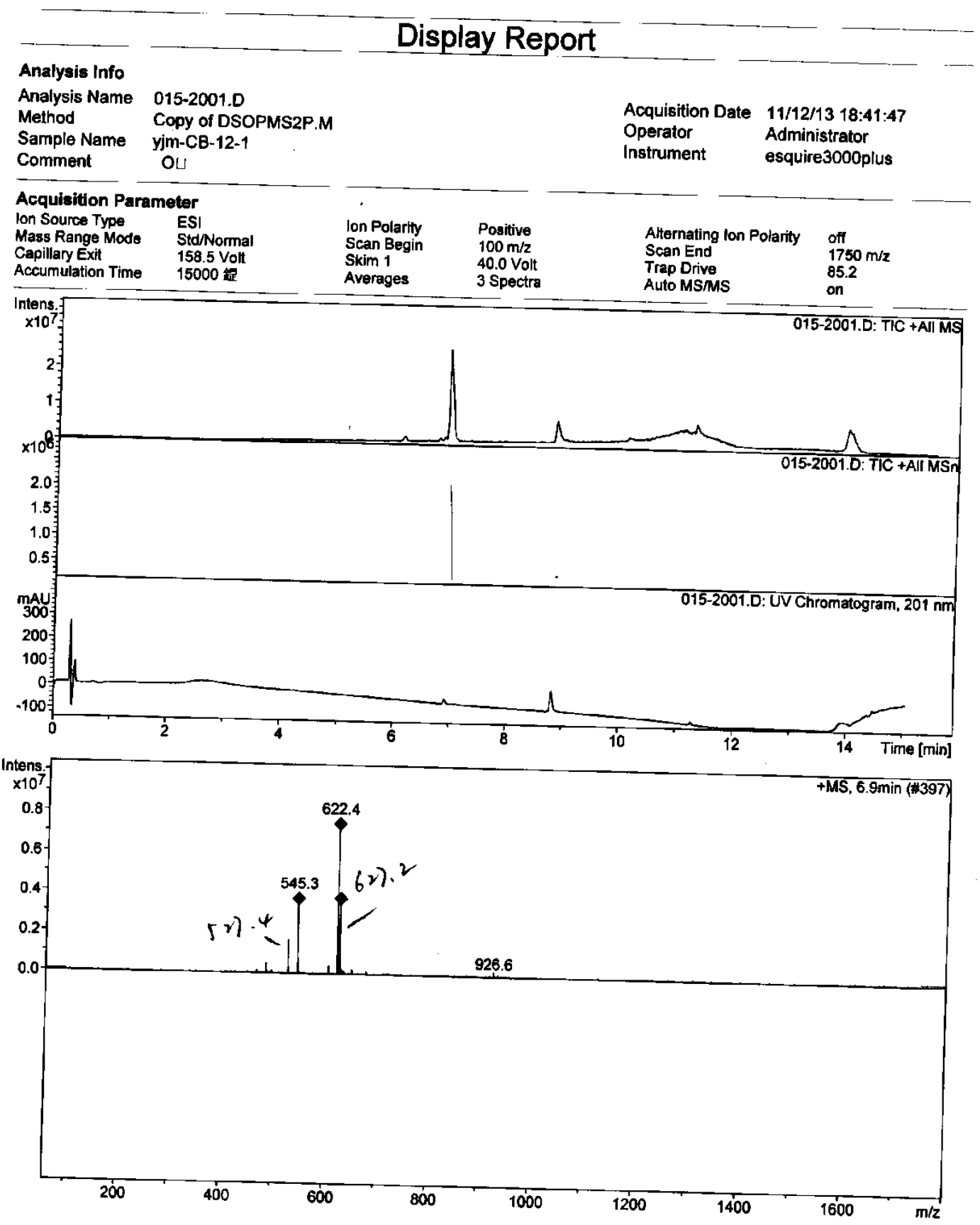

Bruker Daltonics DataAnalysis 3.1 — - printed: $11 / 13 \overline{1} \overline{3}-09: \overline{55: 18}-1$ Page 1 of 1 
Figure S73. ESI(-)MS spectrum of cibacciferin E (9)

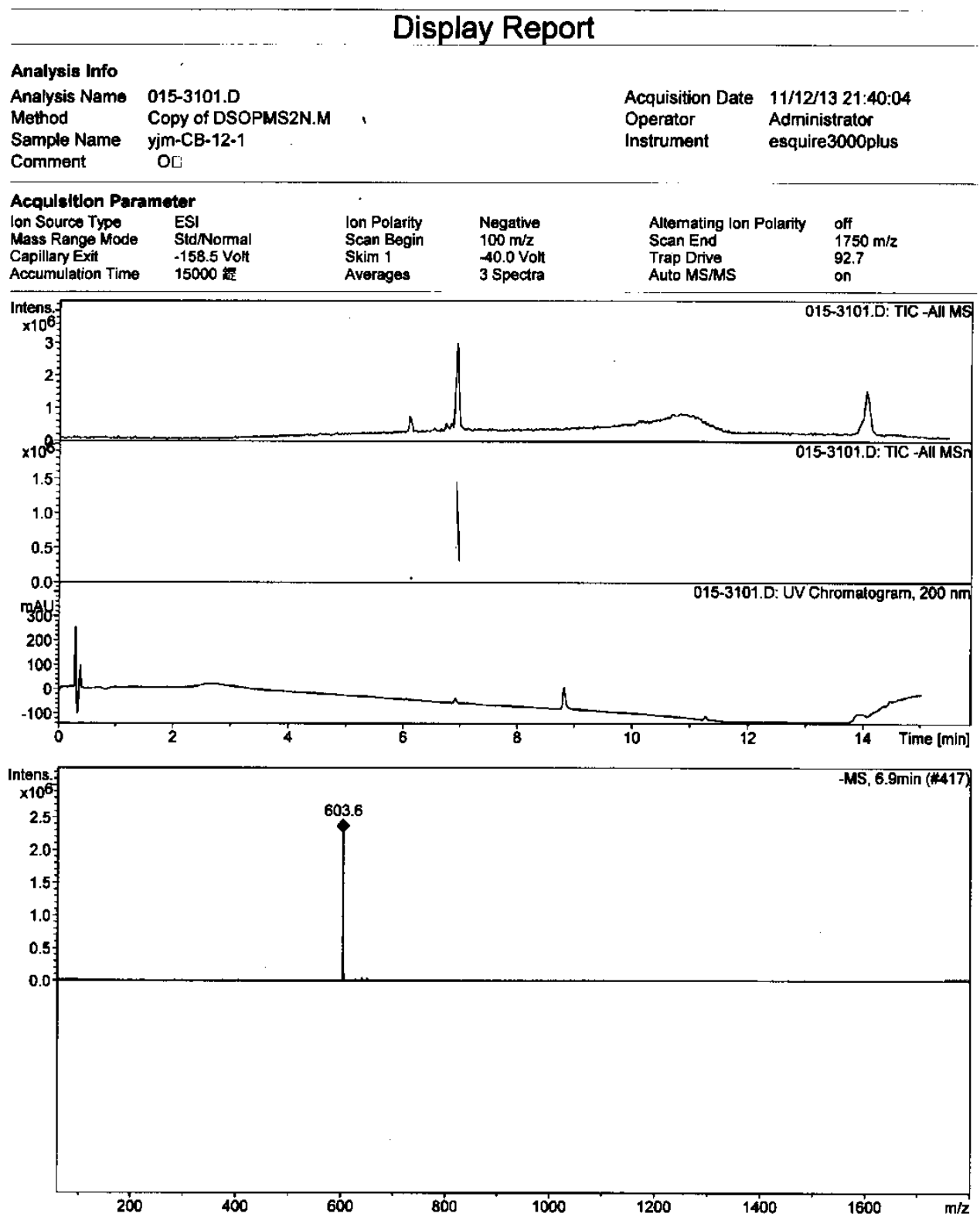


Figure S74. HRESI(+)MS spectrum of cibacciferin E (9)

\section{Elemental Composition Report}

Single Mass Analysis

Tolerance $=3.0$ PPM / DBE: $\min =-1.5, \max =50.0$

Element prediction: Off

Number of isotope peaks used for $\mathrm{i}-\mathrm{FIT}=3$

Monoisotopic Mass, Even Electron Ions

257 formula(e) evaluated with 1 results within limits (up to 50 closest results for each mass)

$\begin{array}{llll}\text { C: } 5-80 & H: 2-120 & \text { O: } 0-20 & \mathrm{Na}: 0-1\end{array}$
CB-12-1
LCT PXE KE324

19-Nov-2013

CB-12-1_111921 (0.423) AM2 (Ar, 10000.0,0.00,1.00); ABS; Cm (9:23)

$$
\text { }
$$

627.2416

$9.050+003$

Minimum:

Maximum:

$\begin{array}{lll}5.0 & 3.0 & -1.5 \\ & & 50.0\end{array}$

Mass Calc. Mass mDa PPM DBE

$627.2416 \quad 627.2417$

$-0.1 \quad-0.2$

11.5

i-FIT

i-FIT (Norm) Formula

627.2417

$\begin{array}{lll}-0.1 & -0.2 \quad 11.5\end{array}$

54.7

0.0

C31 $\mathrm{H} 40 \quad 012 \quad \mathrm{Na}$ 
Figure S75. IR spectrum of cibacciferin E (9)

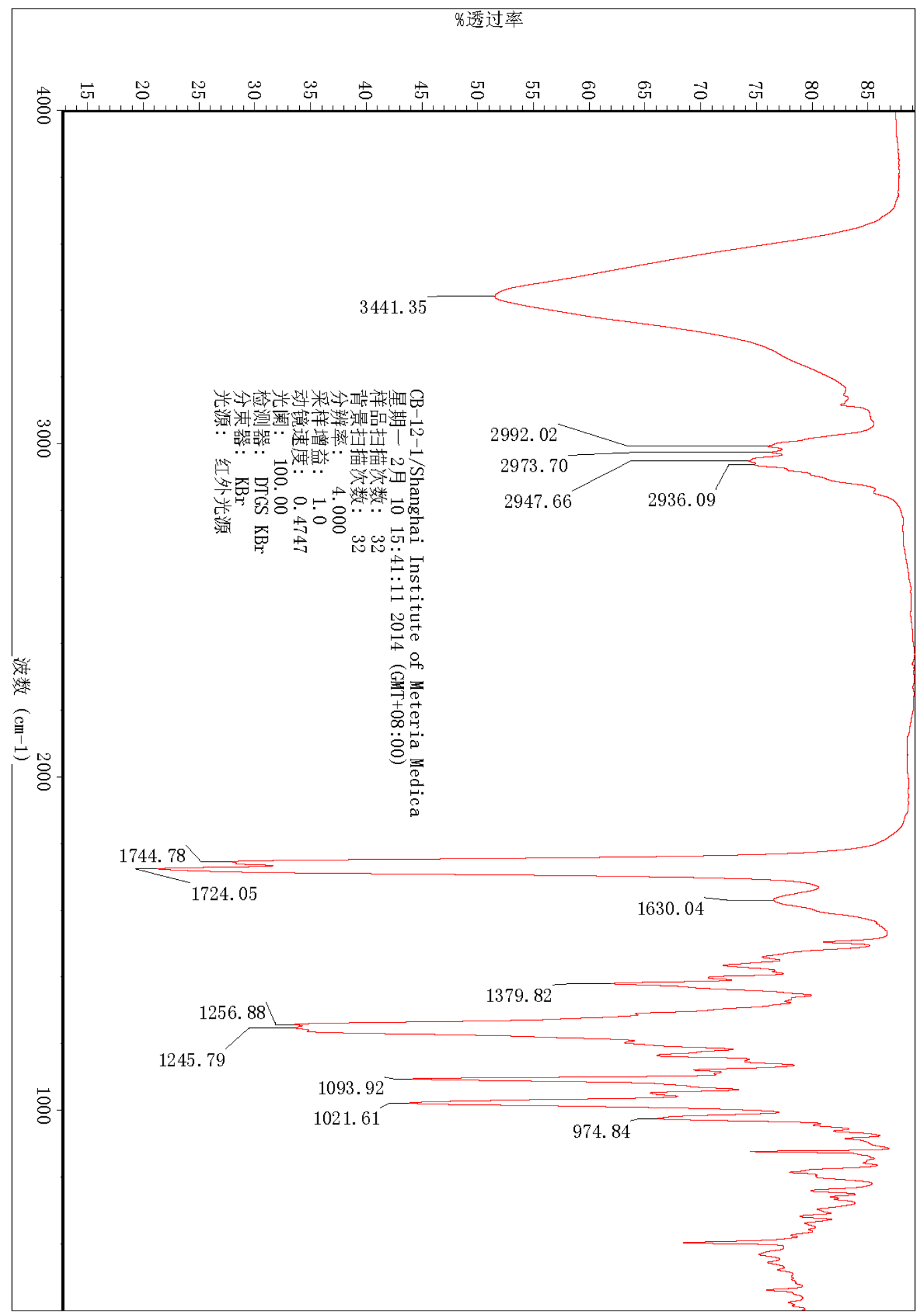


Figure S76. ${ }^{1} \mathrm{H}$ NMR spectrum of $2 \beta$-acetoxycibacciferin $\mathrm{E}(\mathbf{1 0})$ in $\mathrm{CDCl}_{3}$

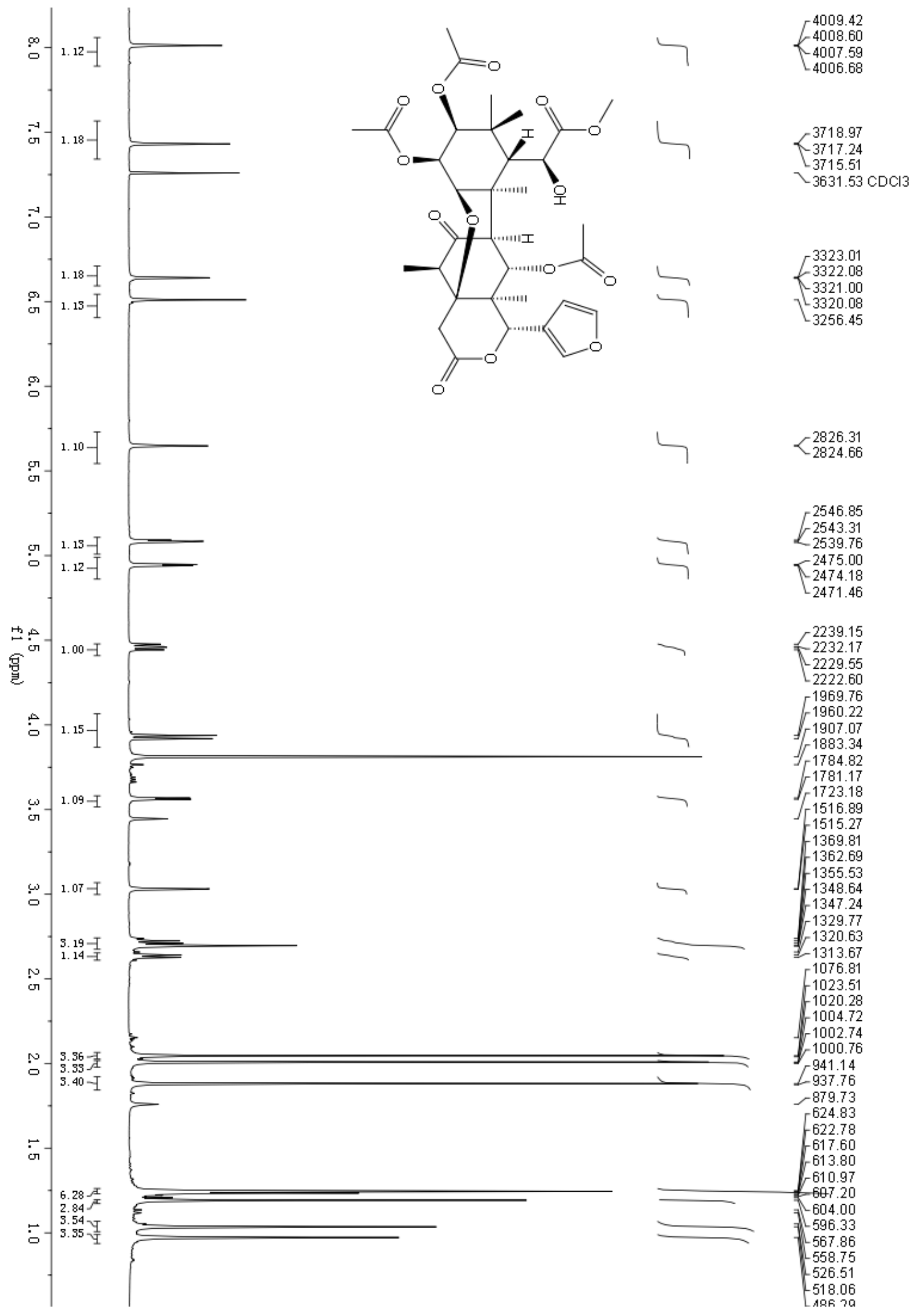


Figure S77. ${ }^{13} \mathrm{C}$ NMR spectrum of $2 \beta$-acetoxycibacciferin $\mathrm{E}(\mathbf{1 0})$ in $\mathrm{CDCl}_{3}$

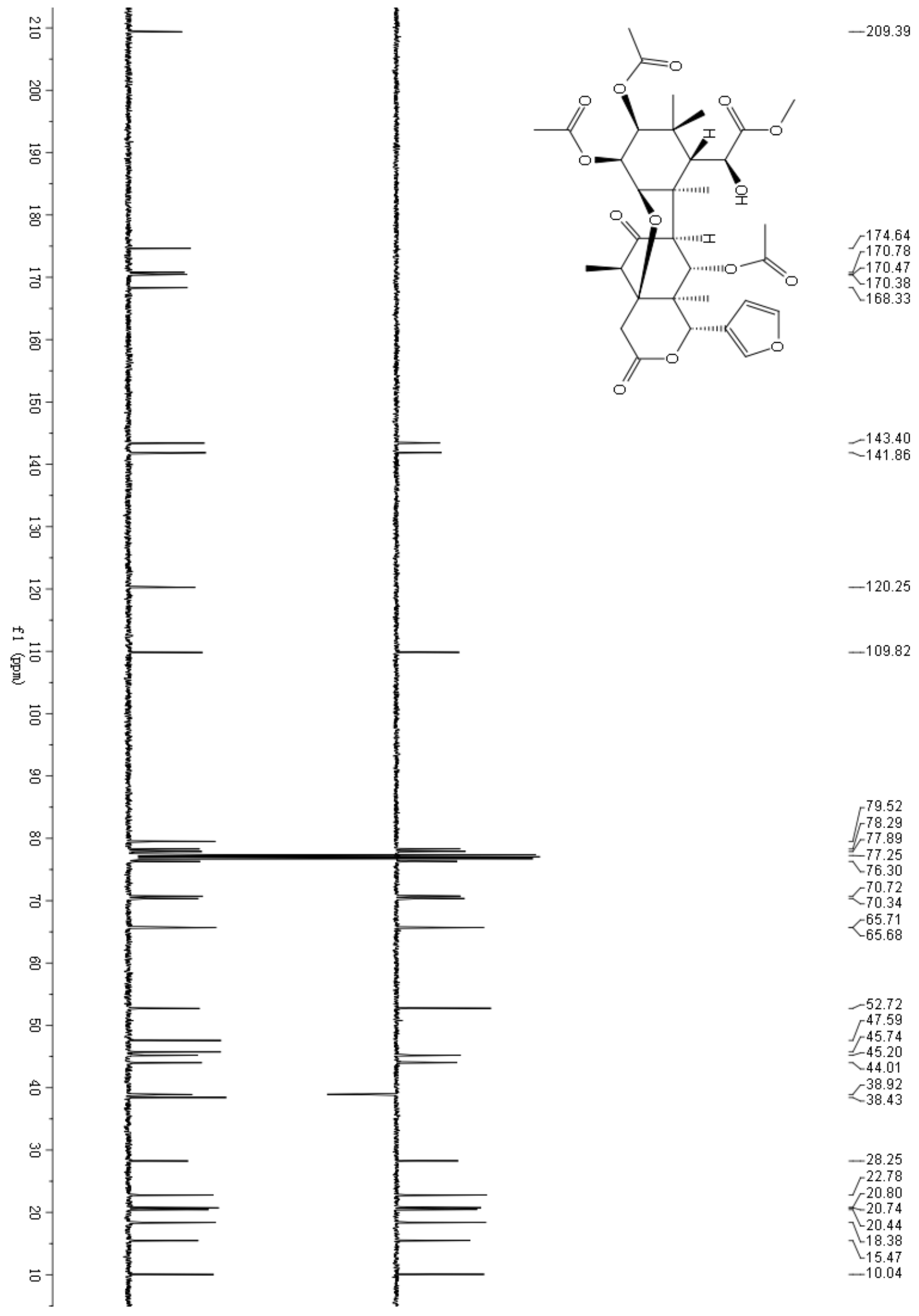


Figure S78. HSQC spectrum of $2 \beta$-acetoxycibacciferin $\mathrm{E}(\mathbf{1 0})$ in $\mathrm{CDCl}_{3}$

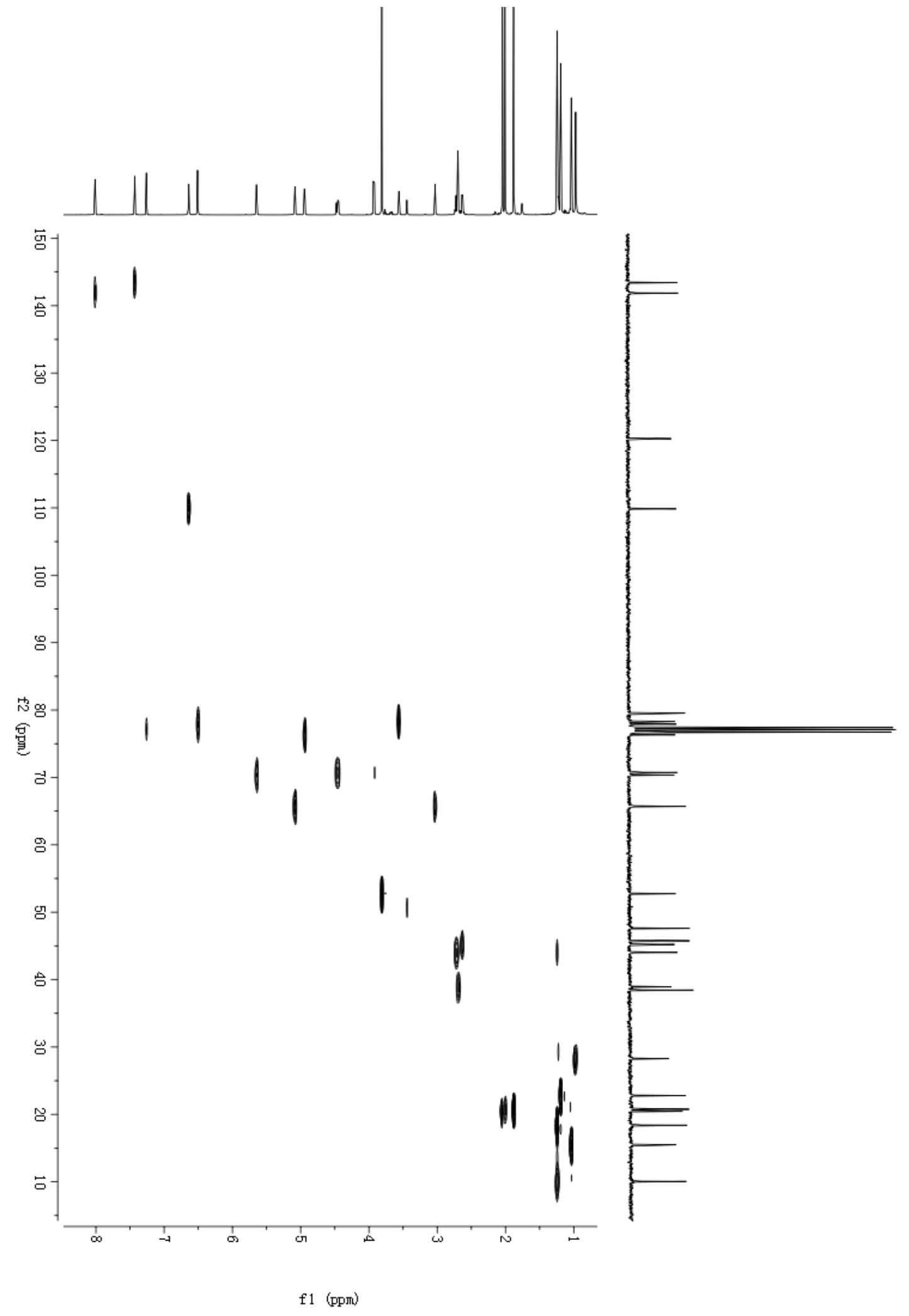


Figure S79. $\mathrm{HMBC}$ spectrum of $2 \beta$-acetoxycibacciferin $\mathrm{E}(\mathbf{1 0})$ in $\mathrm{CDCl}_{3}$

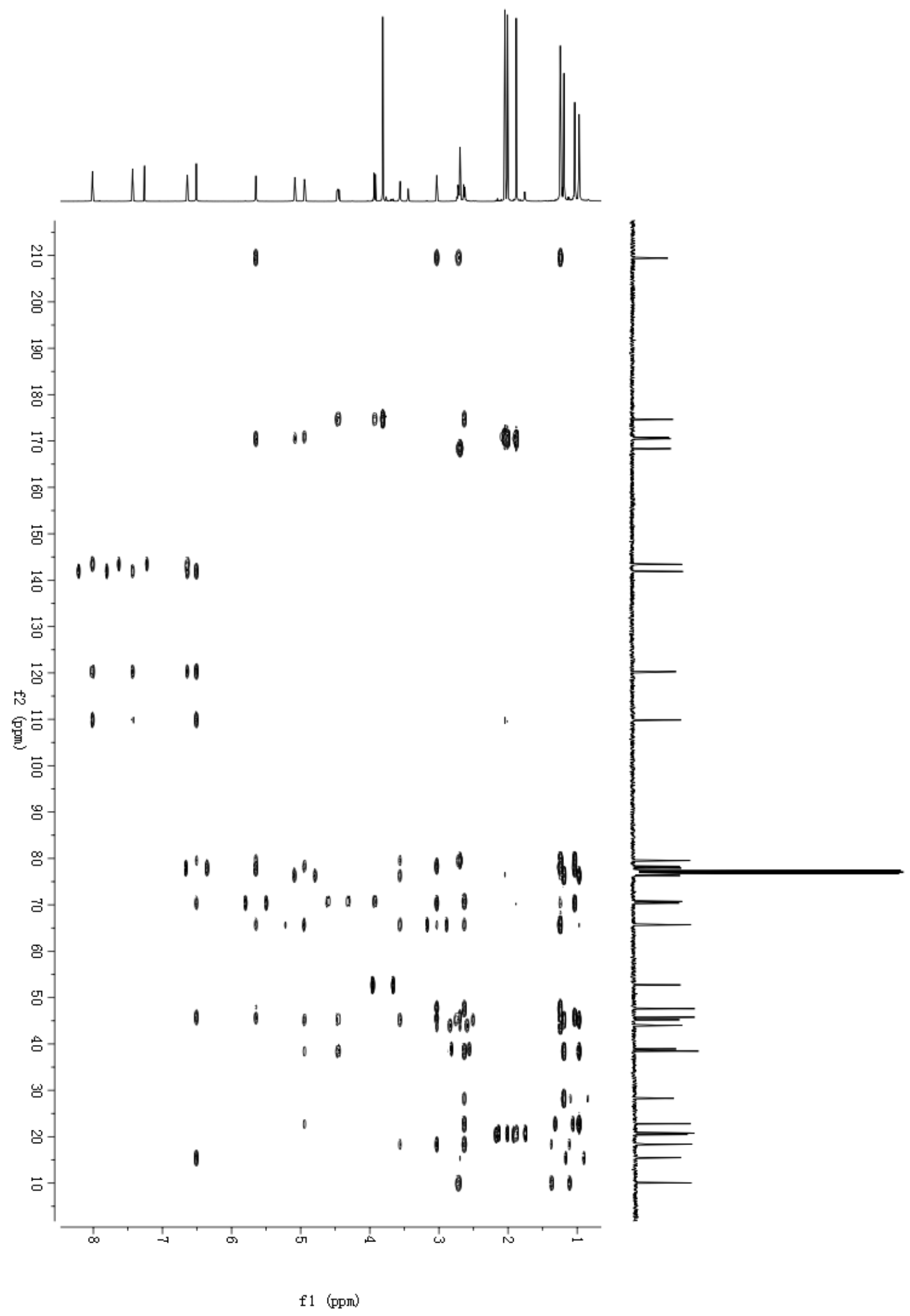


Figure S80. ESI(+)MS spectrum of $2 \beta$-acetoxycibacciferin E (10)

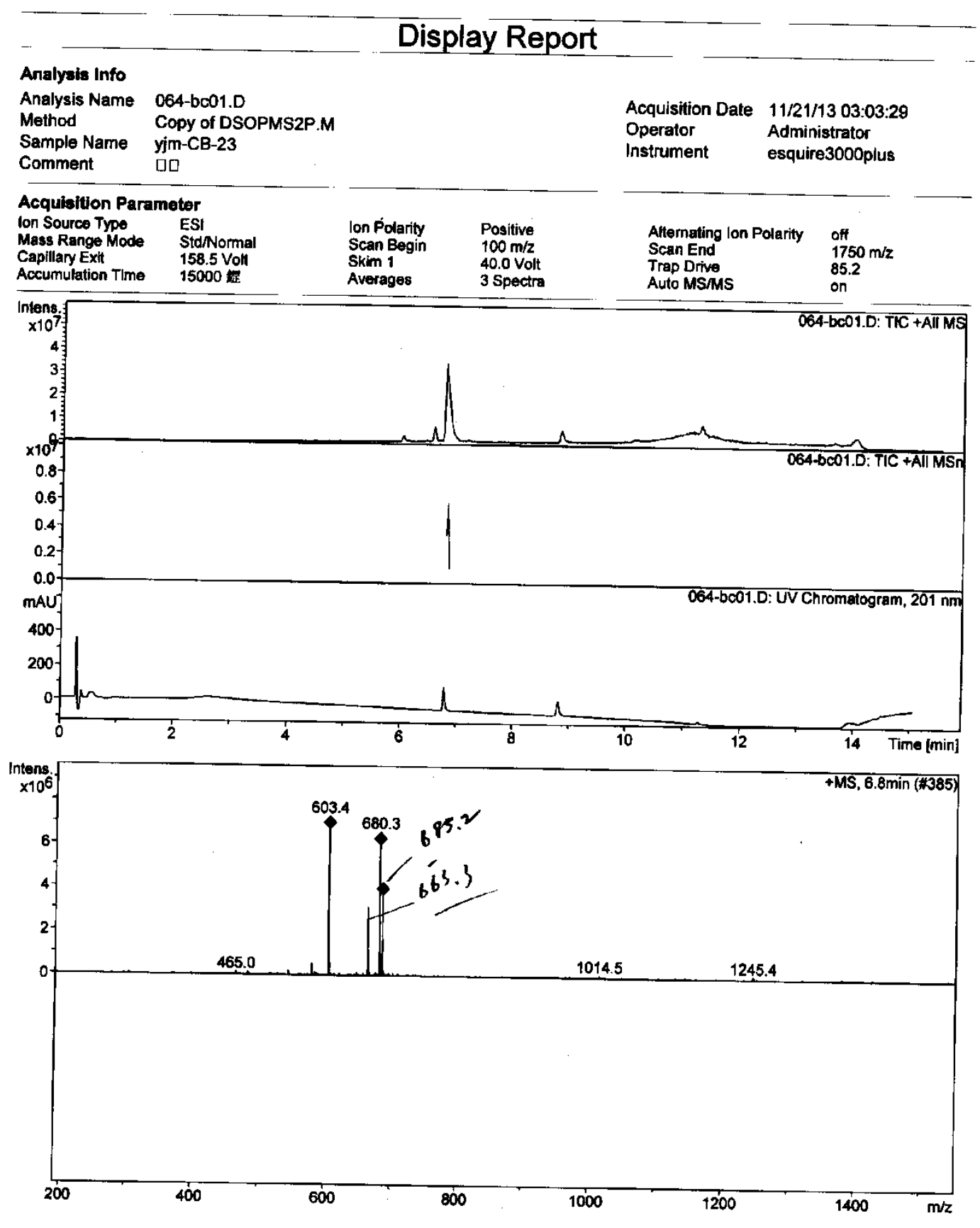


Figure S81. ESI(-)MS spectrum of $2 \beta$-acetoxycibacciferin E (10)

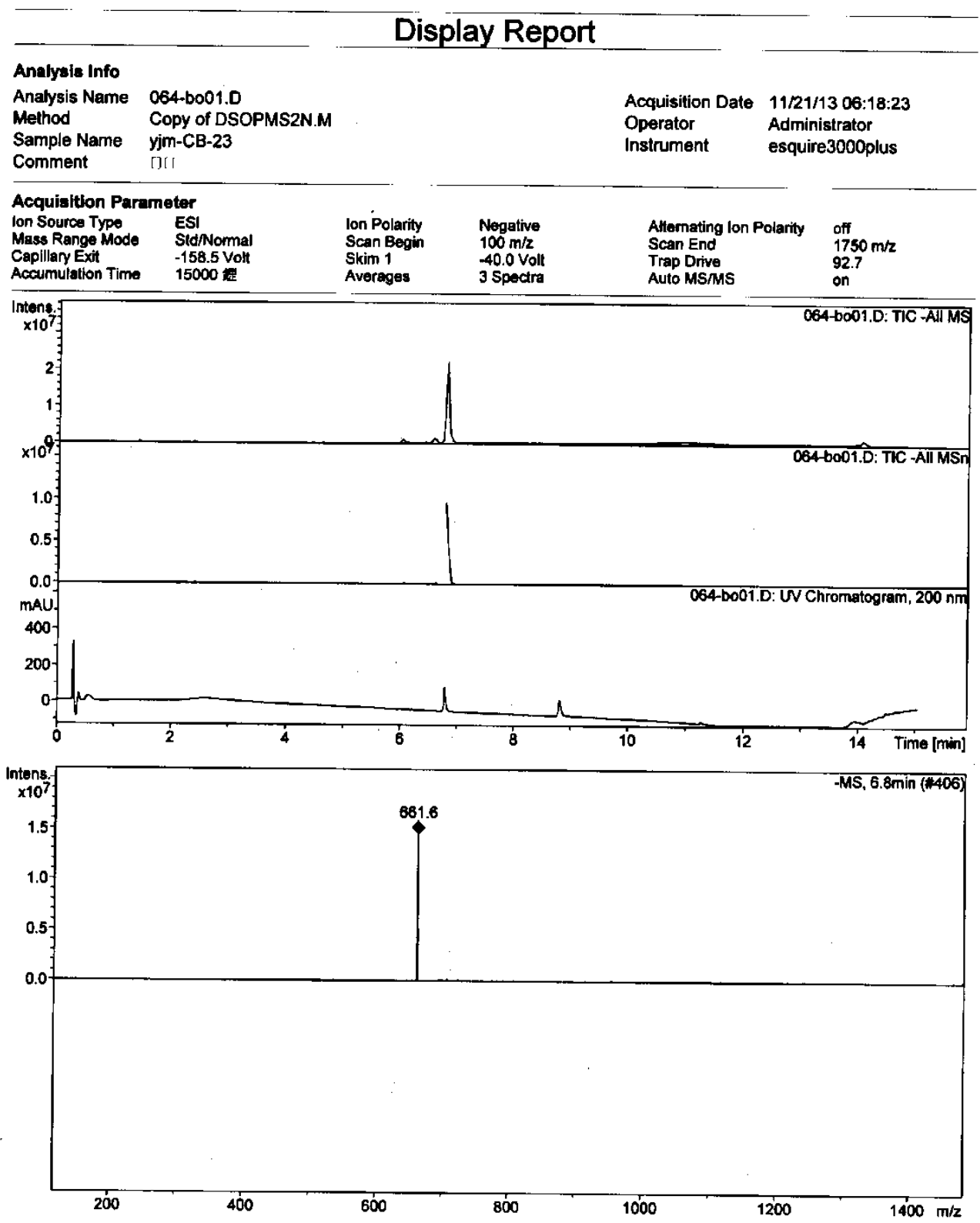


Figure S82. HRESI(+)MS spectrum of $2 \beta$-acetoxycibacciferin E (10)

\section{Elemental Composition Report}

\section{Single Mass Analysis}

Tolerance $=3.0 \mathrm{PPM} / \mathrm{DBE}: \min =-1.5, \max =50.0$

Element prediction: Off

Number of isotope peaks used for $\mathrm{i}-\mathrm{FIT}=3$

Monoisotopic Mass, Even Electron Ions

286 formula(e) evaluated with 1 results within limits (up to 50 closest results for each mass)

Elements Used:

$\begin{array}{lll}\text { C: } 5-80 & \text { H: } 2-120 & \text { O: } 0-20 \quad \mathrm{Na}: 0-1\end{array}$

CB-23 LCT PXE KE324
LB

02-Jan-2014

16:47:01

$\begin{array}{rr}\text { CB-23_20140102 13 (0.247) AM2 (Ar, 10000.0,0.00,1.00); ABS; Cm (10:23) } & 1: \text { TOF MS ES+ } \\ 1.89 \mathrm{e}+003\end{array}$

100

685.2471

:

$\%$

686.2504
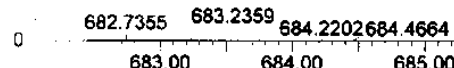

$687.2493 \quad 688.2540,689.3076,690.3077690 .5471 \mathrm{~m} / \mathrm{z}$

Minimum:

Maxinum:

$5.0 \quad-1.5$

IDA

$\begin{array}{lllllllllll}685.2471 & 685.2472 & -0.1 & -0.1 & 12.5 & 15.8 & 0.0 & \mathrm{C} 33 & \mathrm{H} 42 & 014 & \mathrm{Na}\end{array}$ 
Figure S83. IR spectrum of $2 \beta$-acetoxycibacciferin E (10)

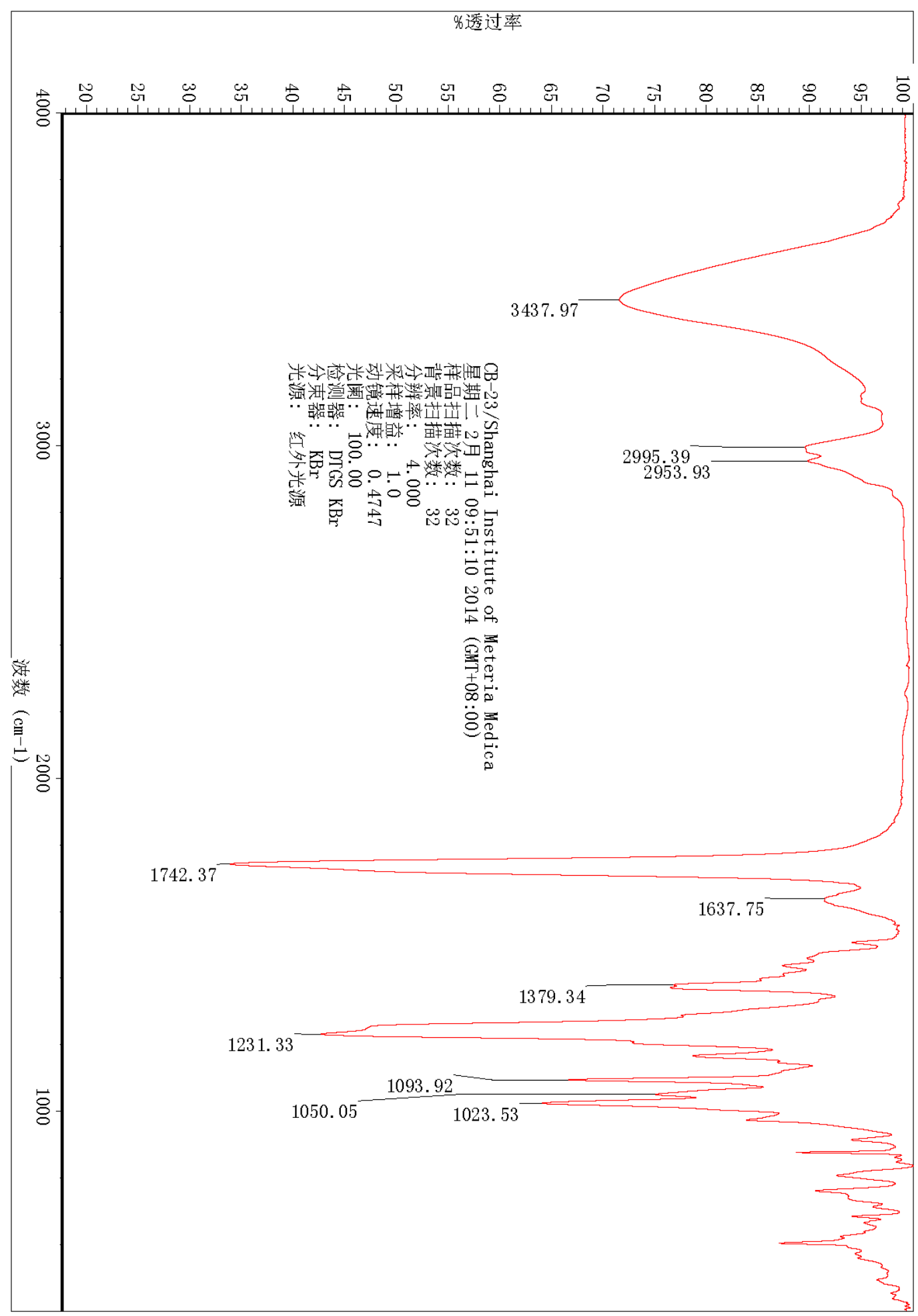


Figure S84. ${ }^{1} \mathrm{H}$ NMR spectrum of cibacciferin $\mathrm{F}(11)$ in $\mathrm{CDCl}_{3}$

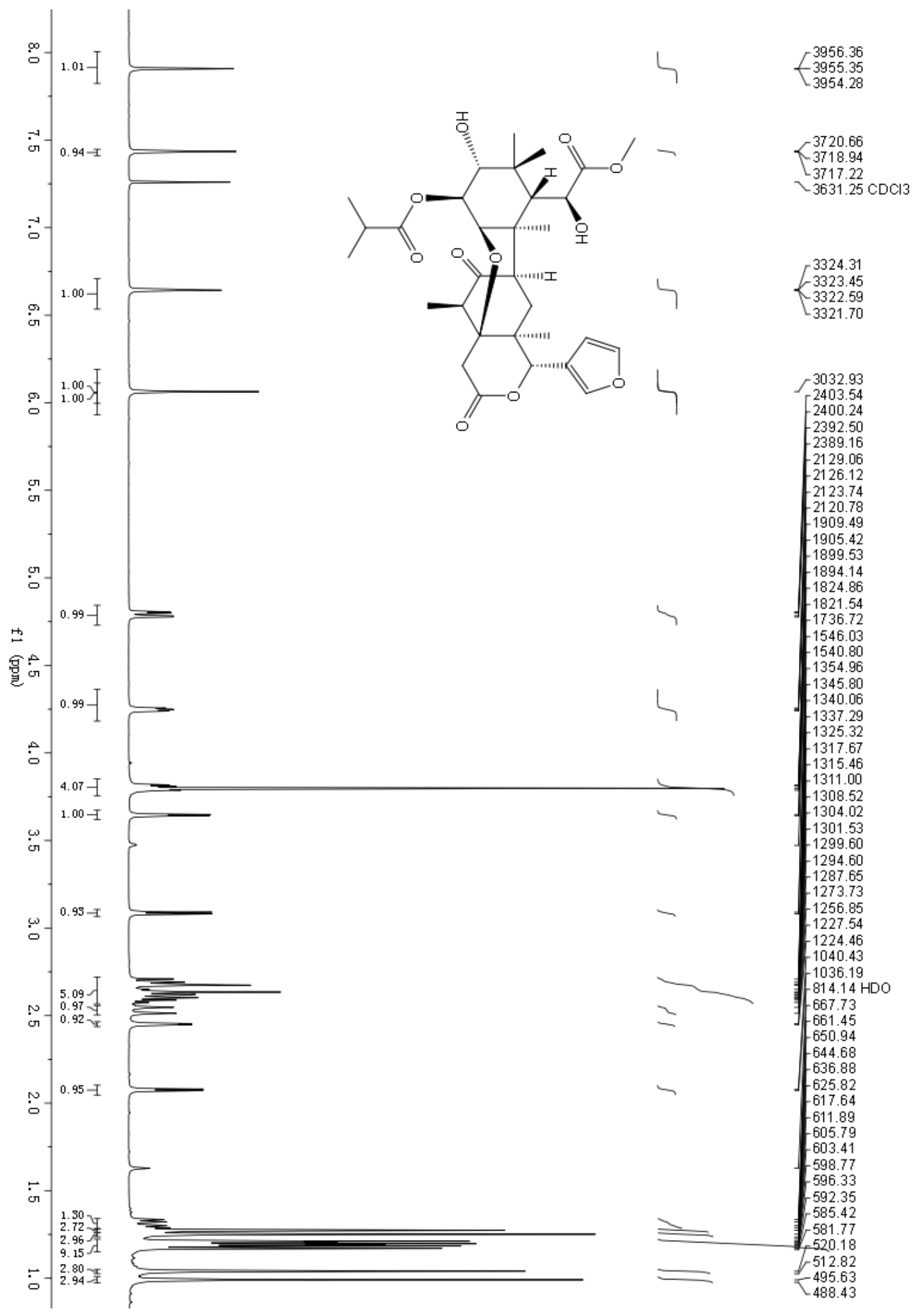


Figure S85. ${ }^{13} \mathrm{C}$ NMR spectrum of cibacciferin $\mathrm{F}(\mathbf{1 1})$ in $\mathrm{CDCl}_{3}$

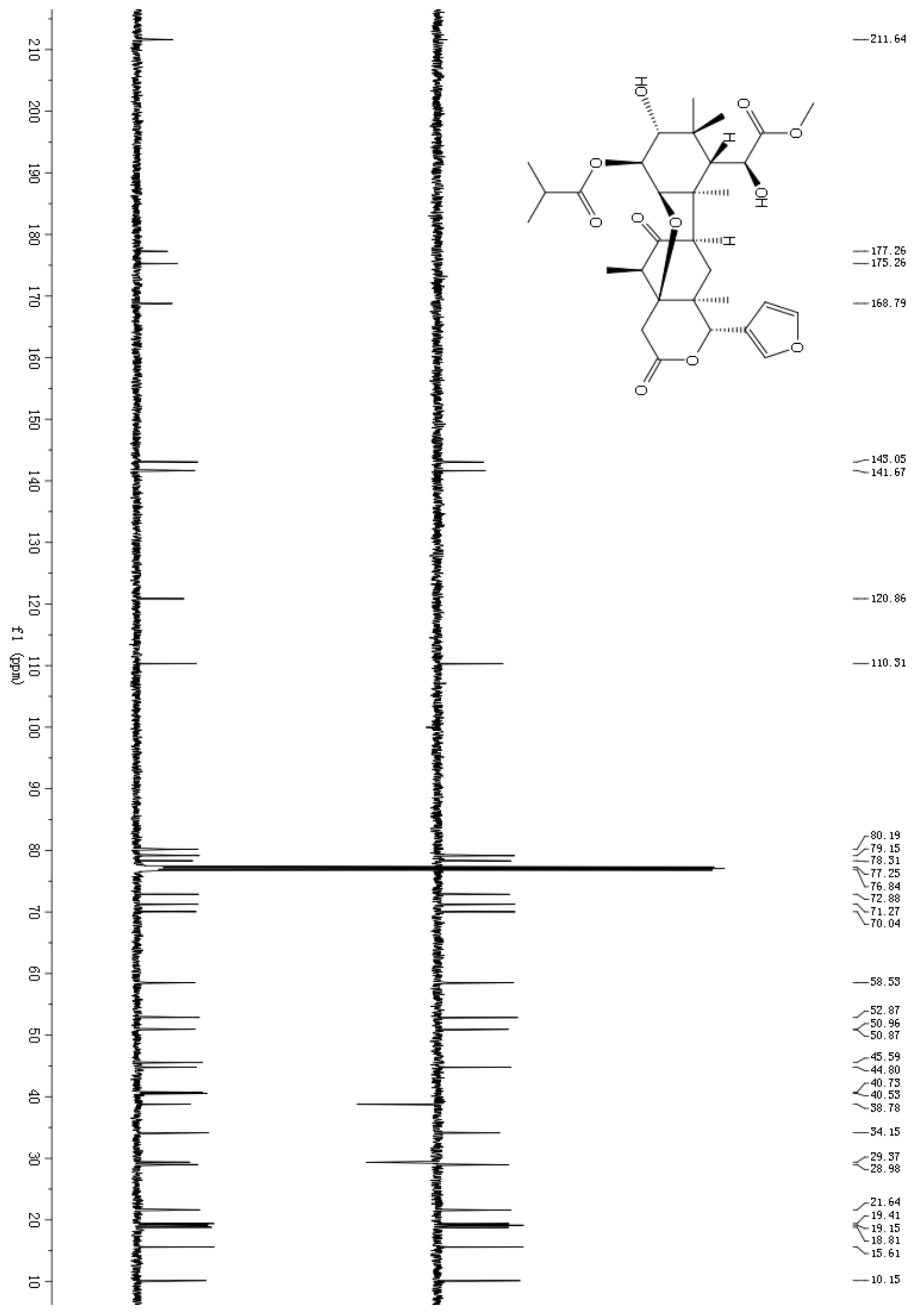


Figure S86. HSQC spectrum of cibacciferin $\mathrm{F}(\mathbf{1 1})$ in $\mathrm{CDCl}_{3}$

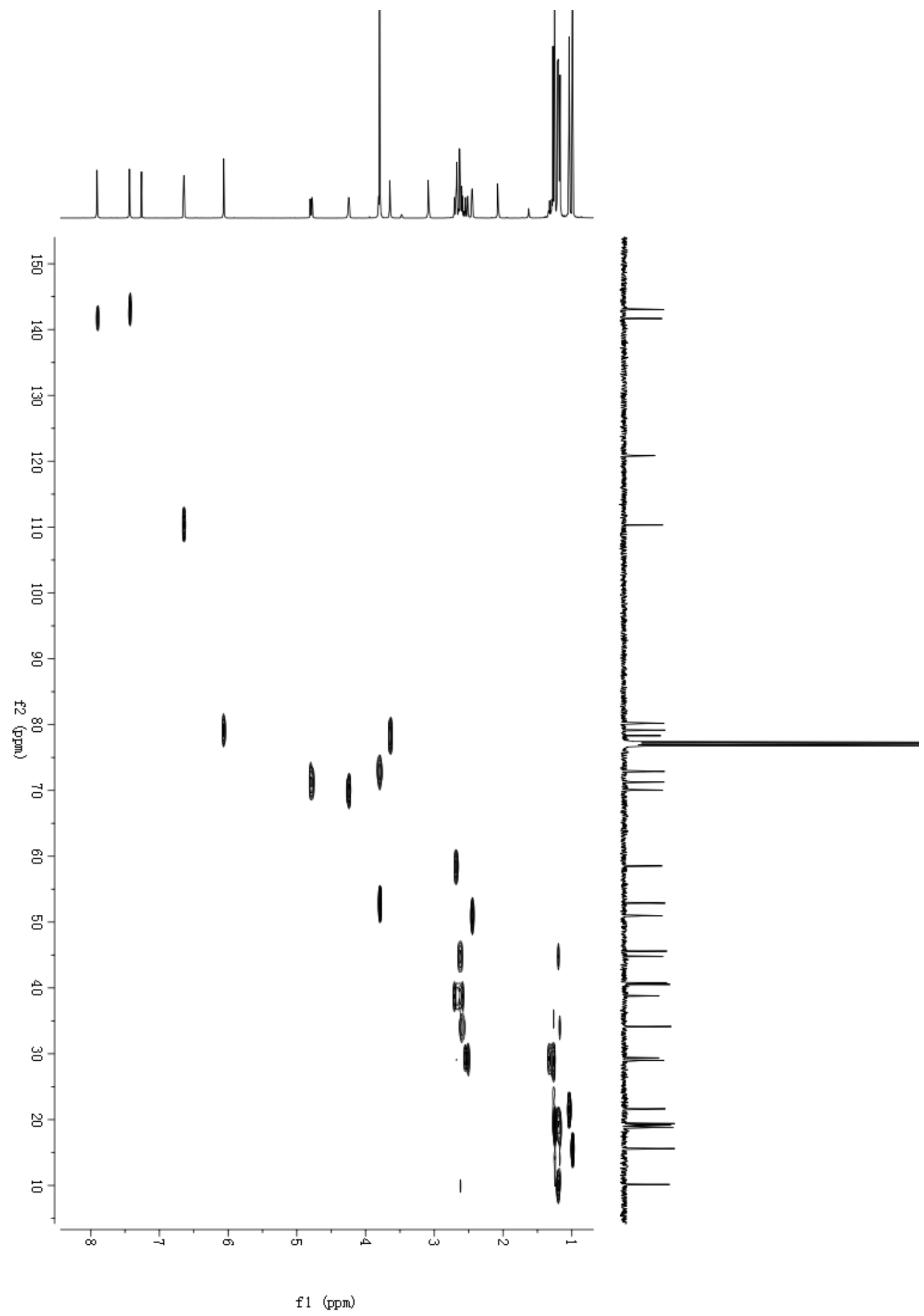


Figure S87. HMBC spectrum of cibacciferin $\mathrm{F}(\mathbf{1 1})$ in $\mathrm{CDCl}_{3}$

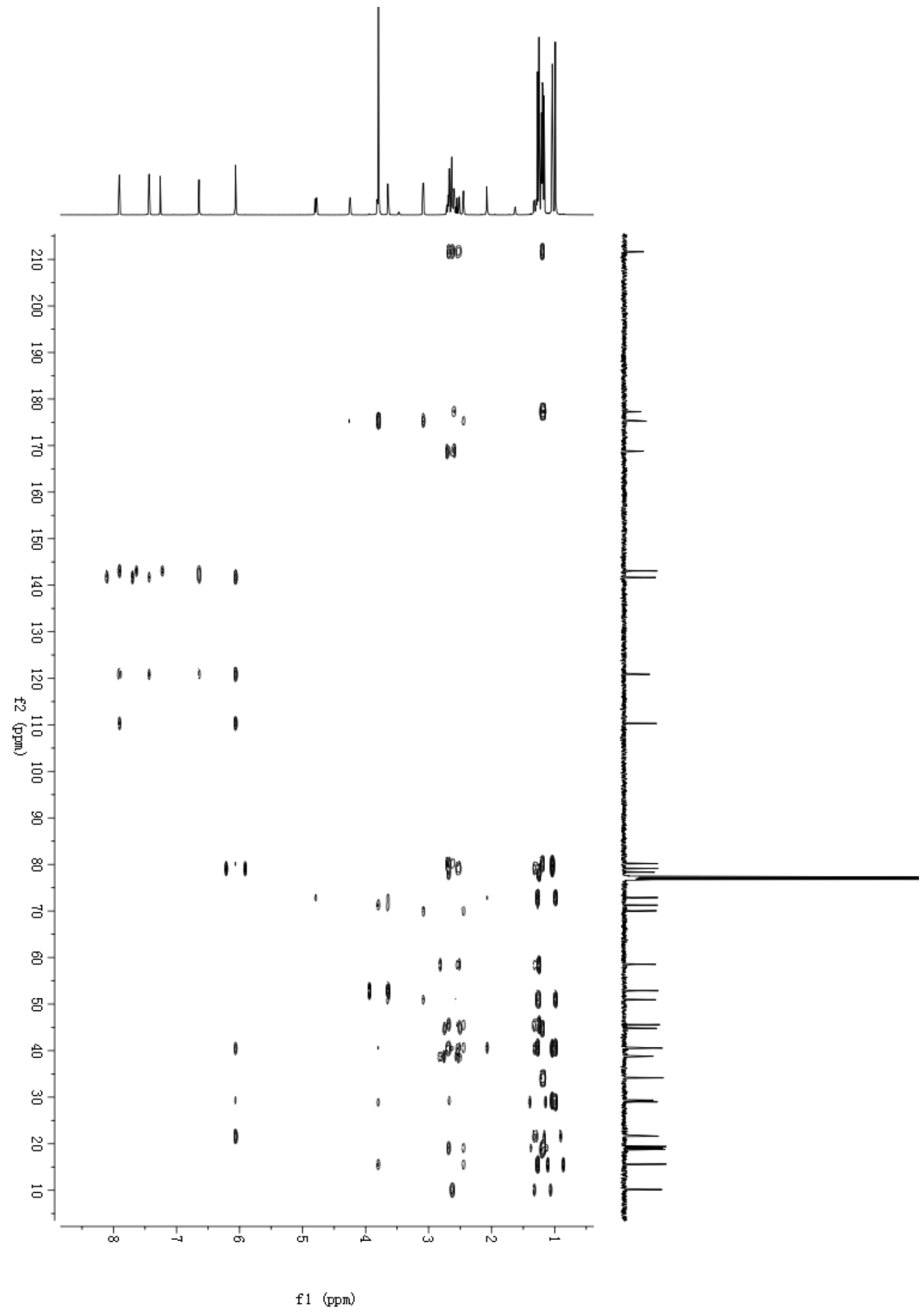


Figure S88. ROESY spectrum of cibacciferin $\mathrm{F}(\mathbf{1 1})$ in $\mathrm{CDCl}_{3}$

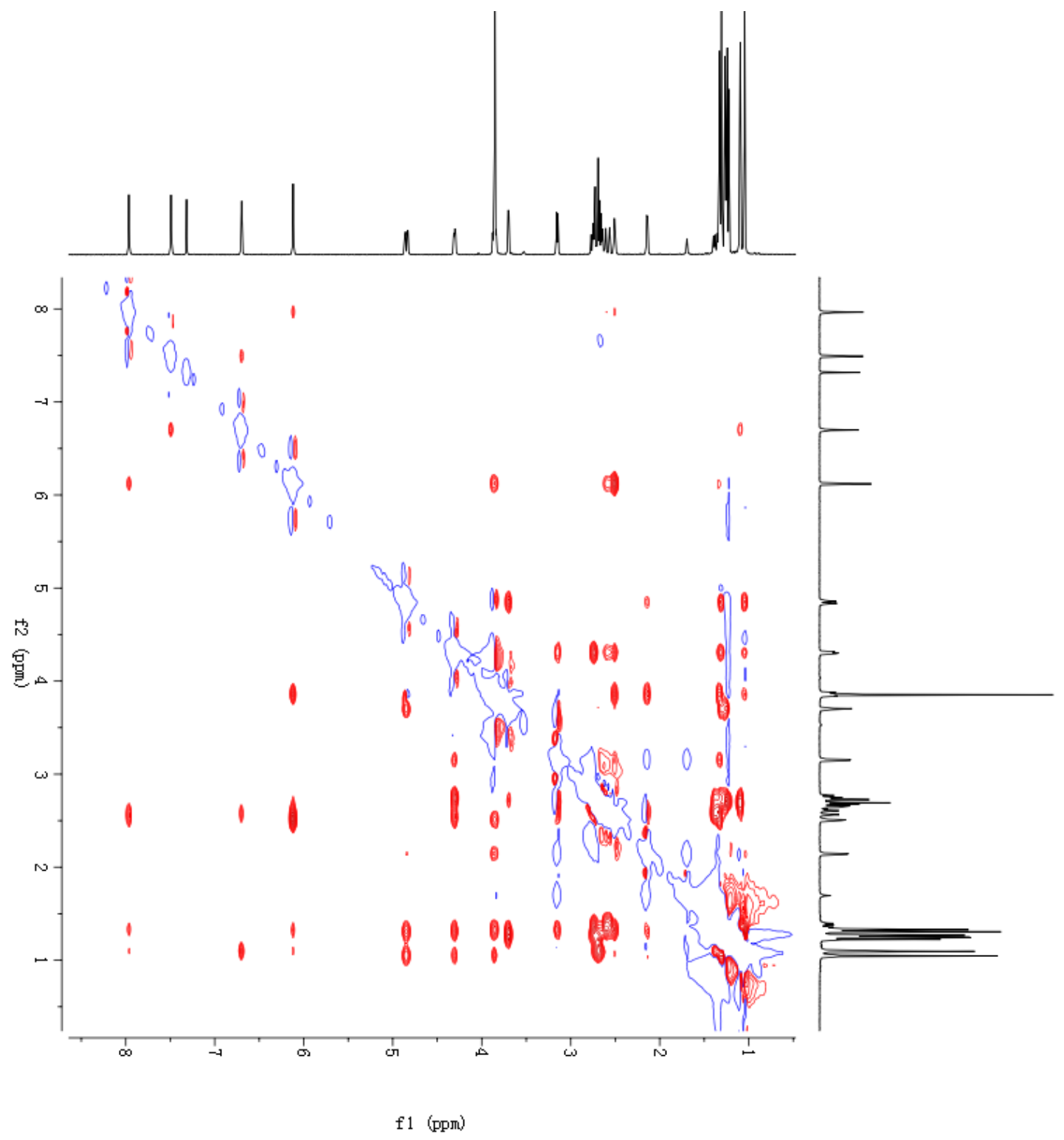


Figure S89. ESI(+)MS spectrum of cibacciferin F (11)

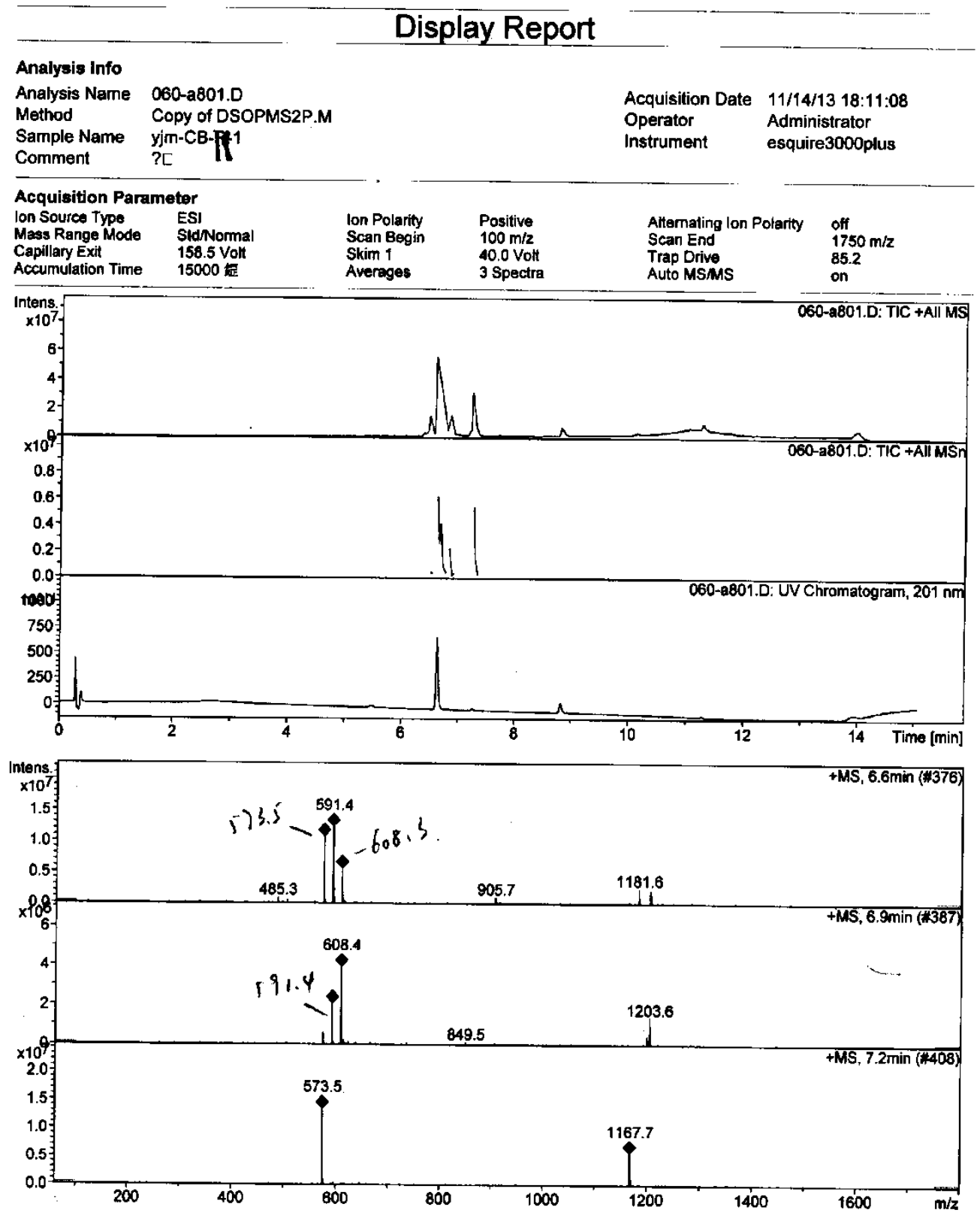


Figure S90. ESI(-)MS spectrum of cibacciferin F (11)

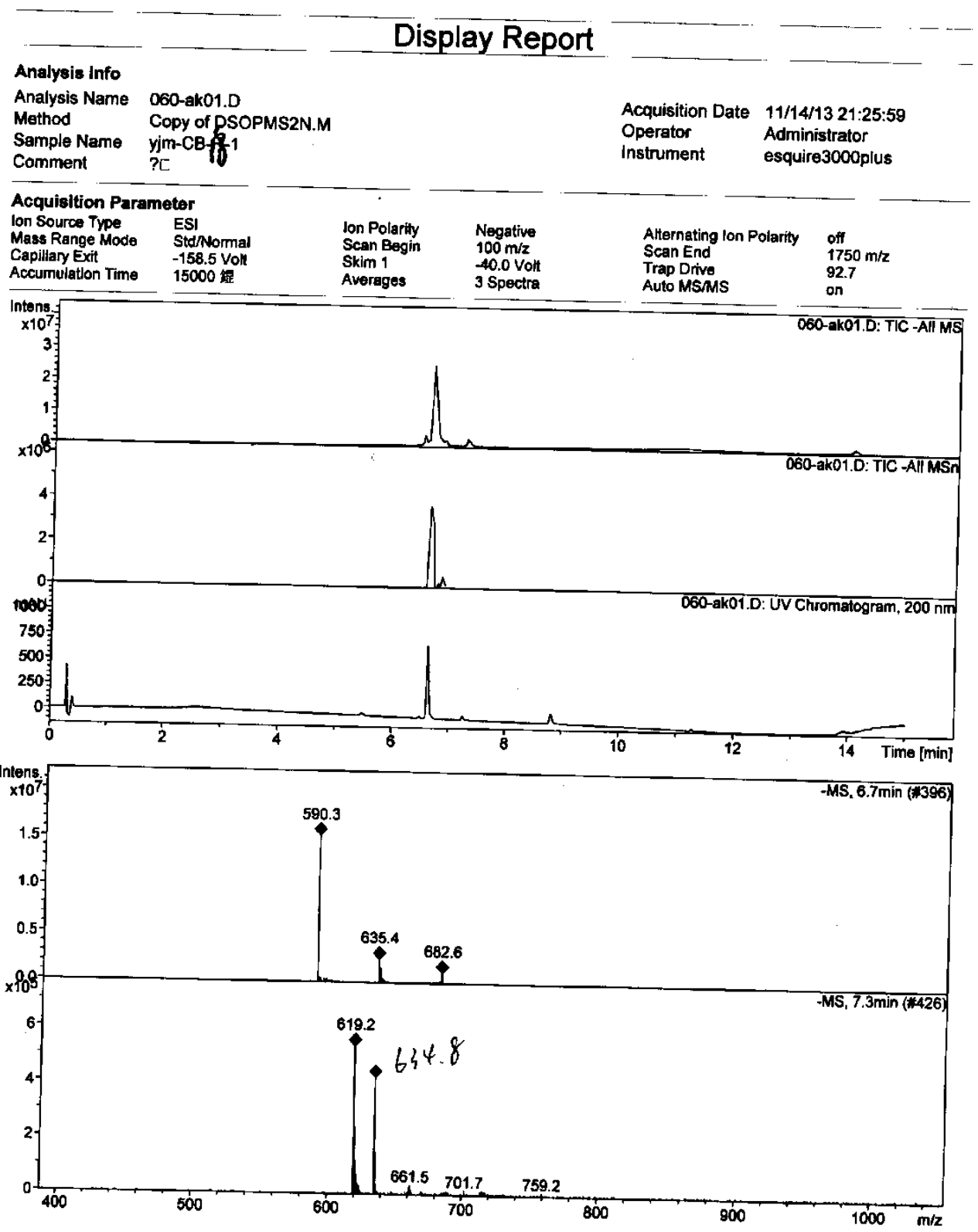

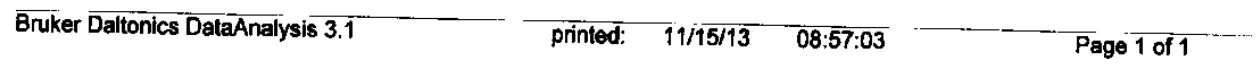


Figure S91. HRESI(+)MS spectrum of cibacciferin F (11)

Elemental Composition Report

Page 1

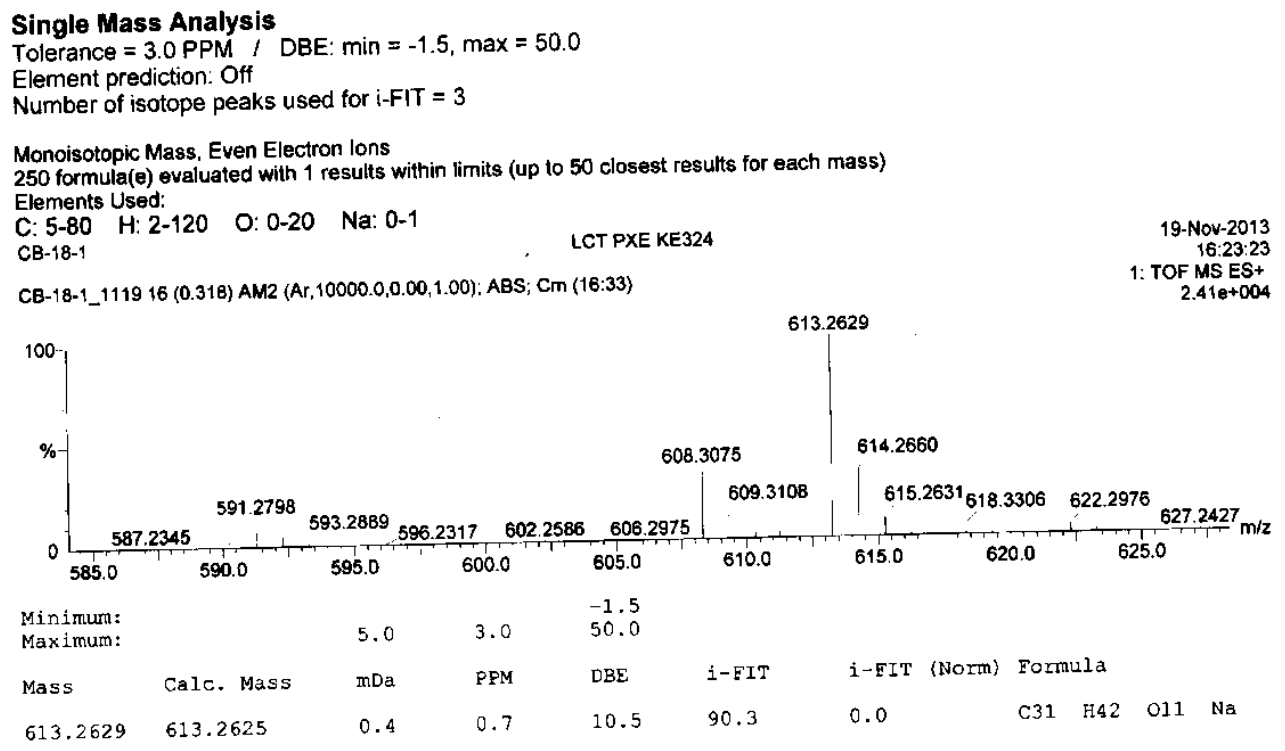


Figure S92. IR spectrum of cibacciferin F (11)

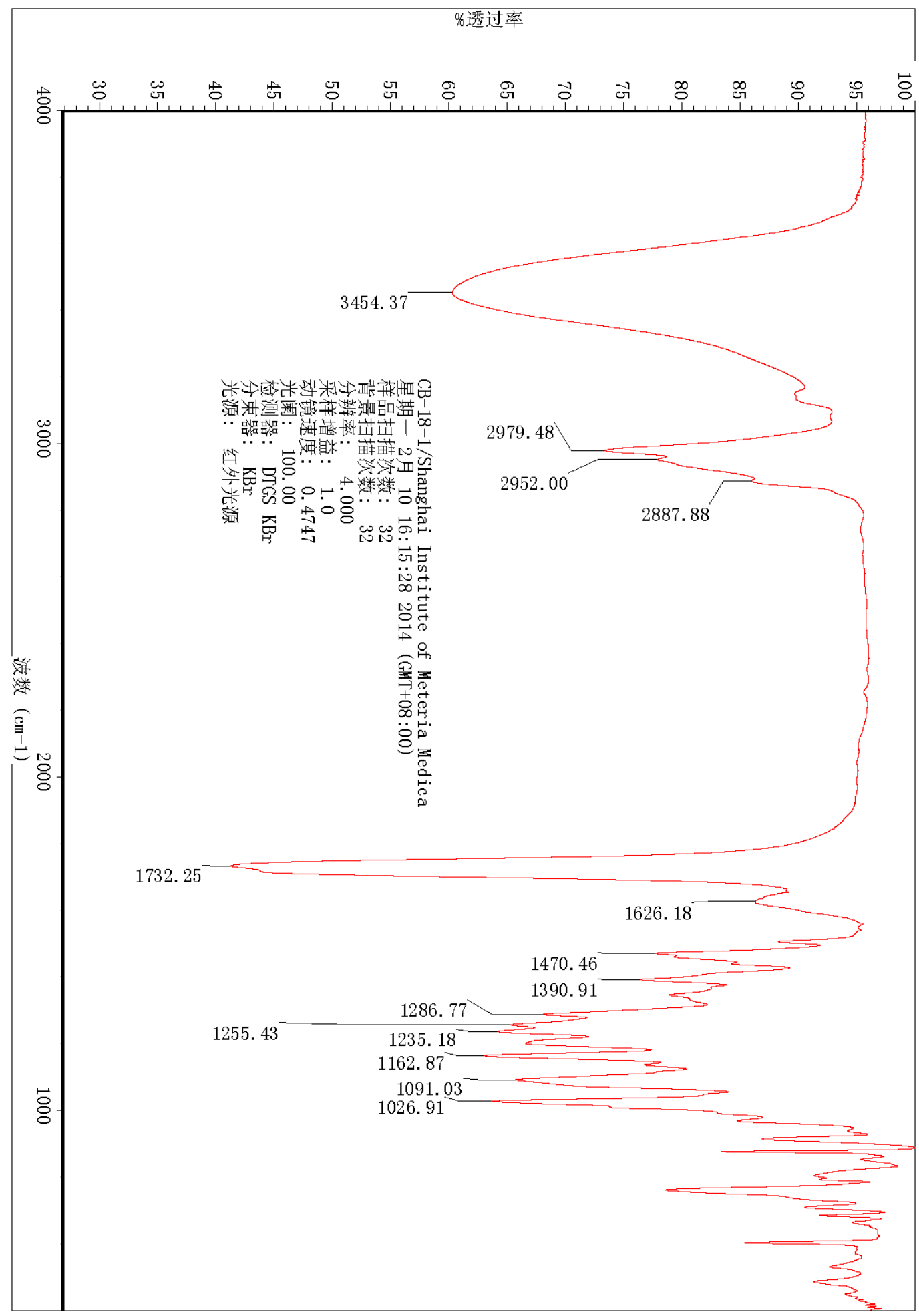


Figure S93. ${ }^{1} \mathrm{H}$ NMR spectrum of 6-dehydroxycibacciferin $\mathrm{F}(\mathbf{1 2})$ in $\mathrm{CDCl}_{3}$

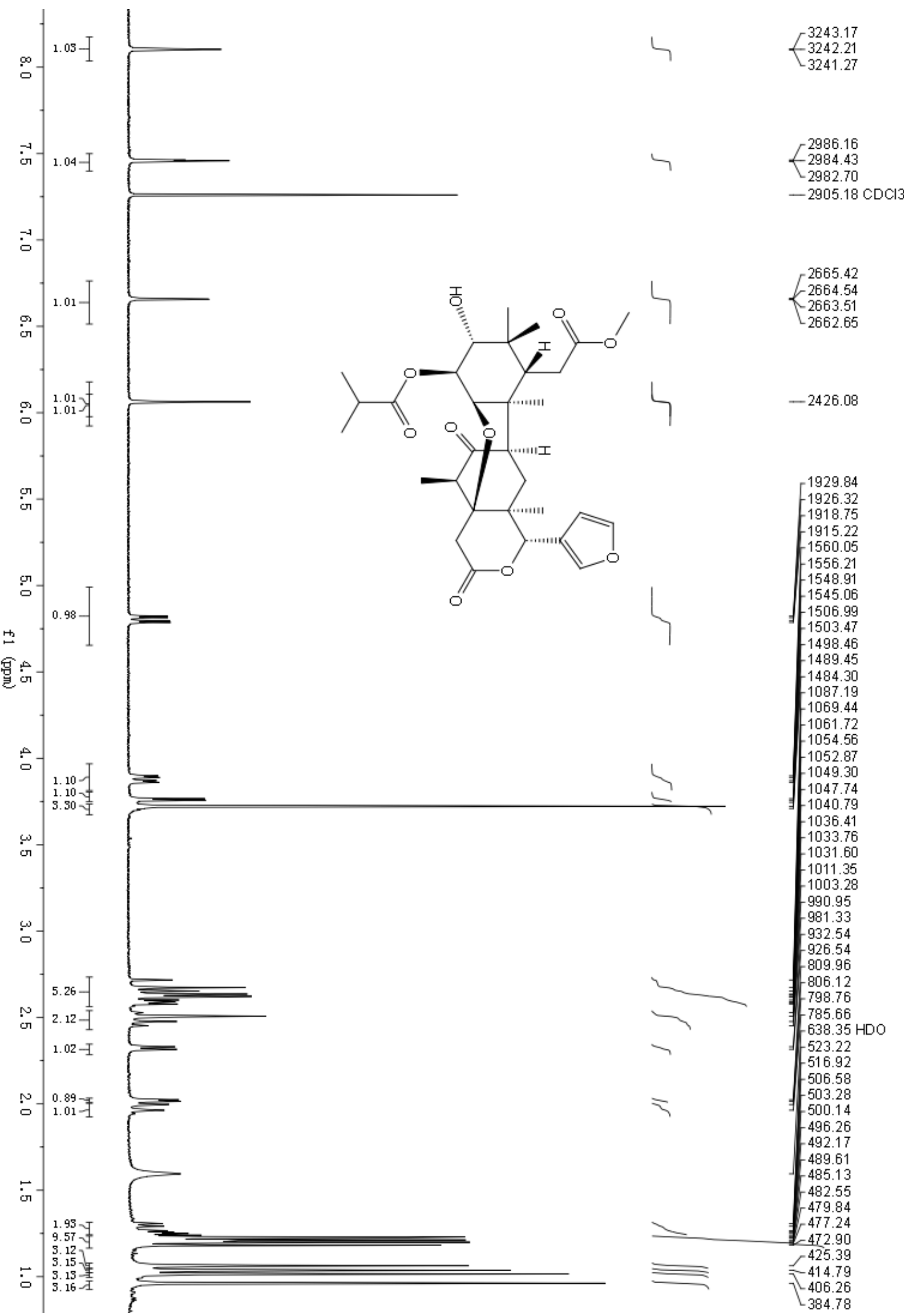


Figure S94. ${ }^{13} \mathrm{C}$ NMR spectrum of 6-dehydroxycibacciferin $\mathrm{F}(\mathbf{1 2})$ in $\mathrm{CDCl}_{3}$

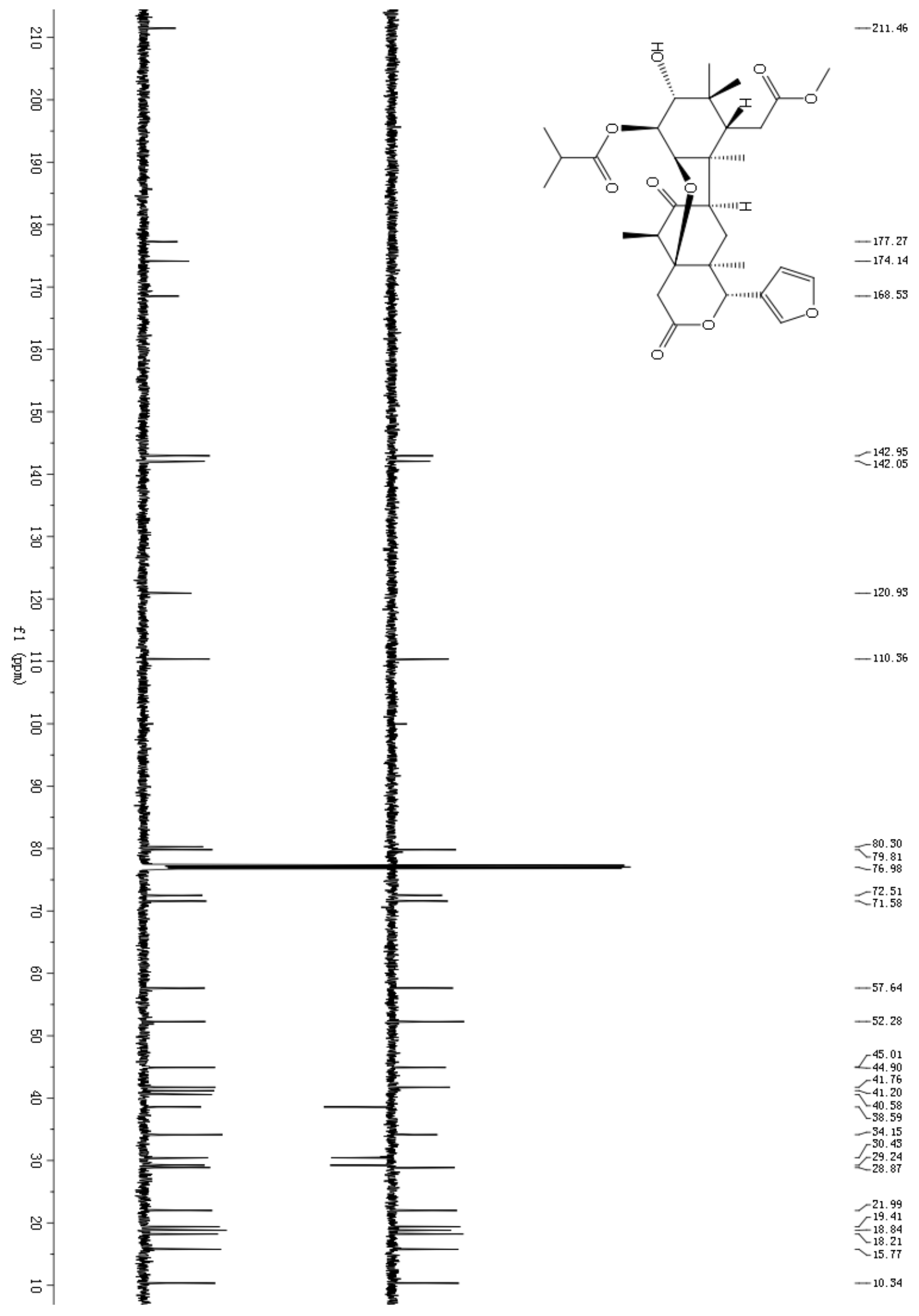


Figure S95. HSQC spectrum of 6-dehydroxycibacciferin $\mathrm{F}$ (12) in $\mathrm{CDCl}_{3}$

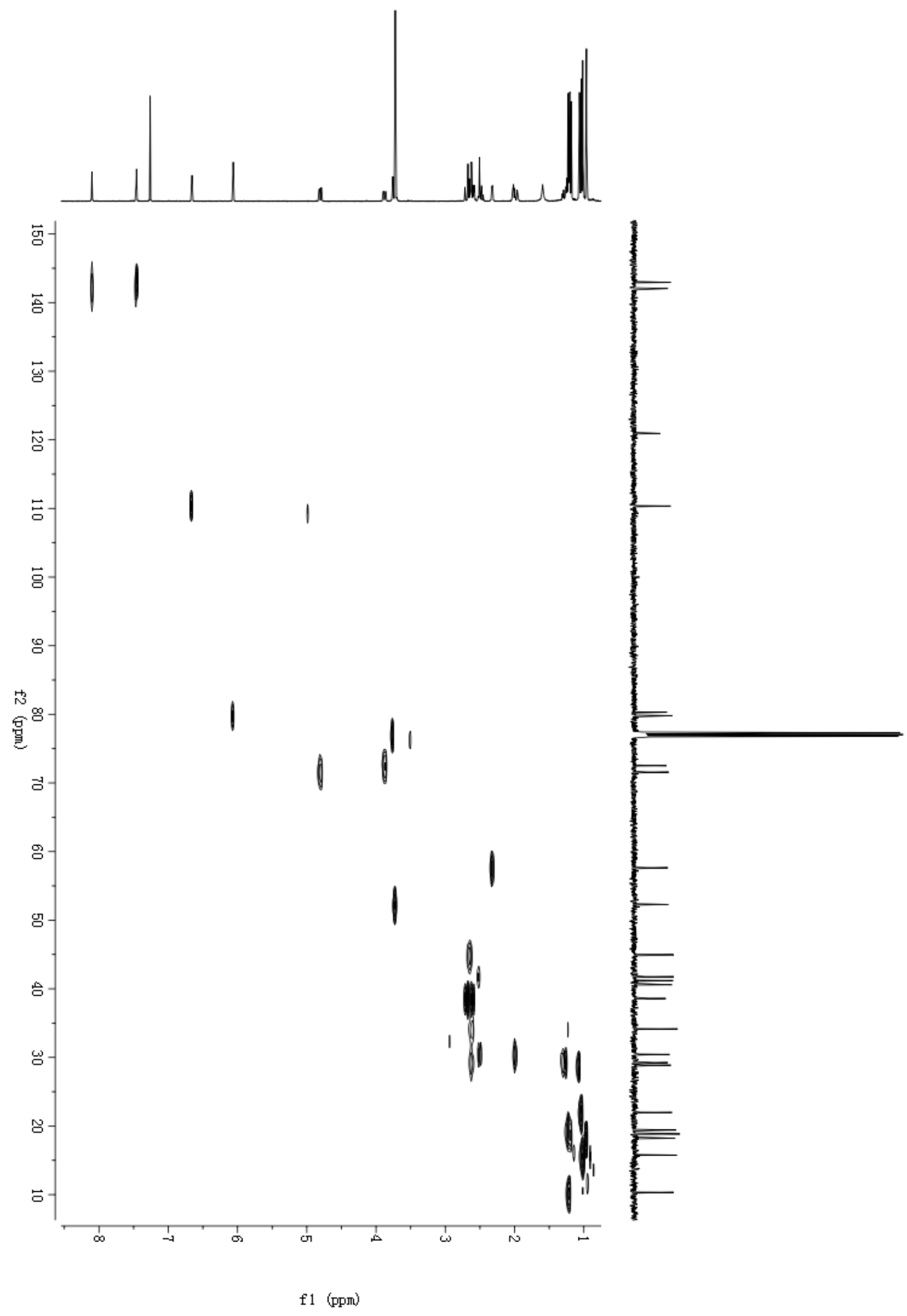


Figure S96. $\mathrm{HMBC}$ spectrum of 6-dehydroxycibacciferin $\mathrm{F}$ (12) in $\mathrm{CDCl}_{3}$

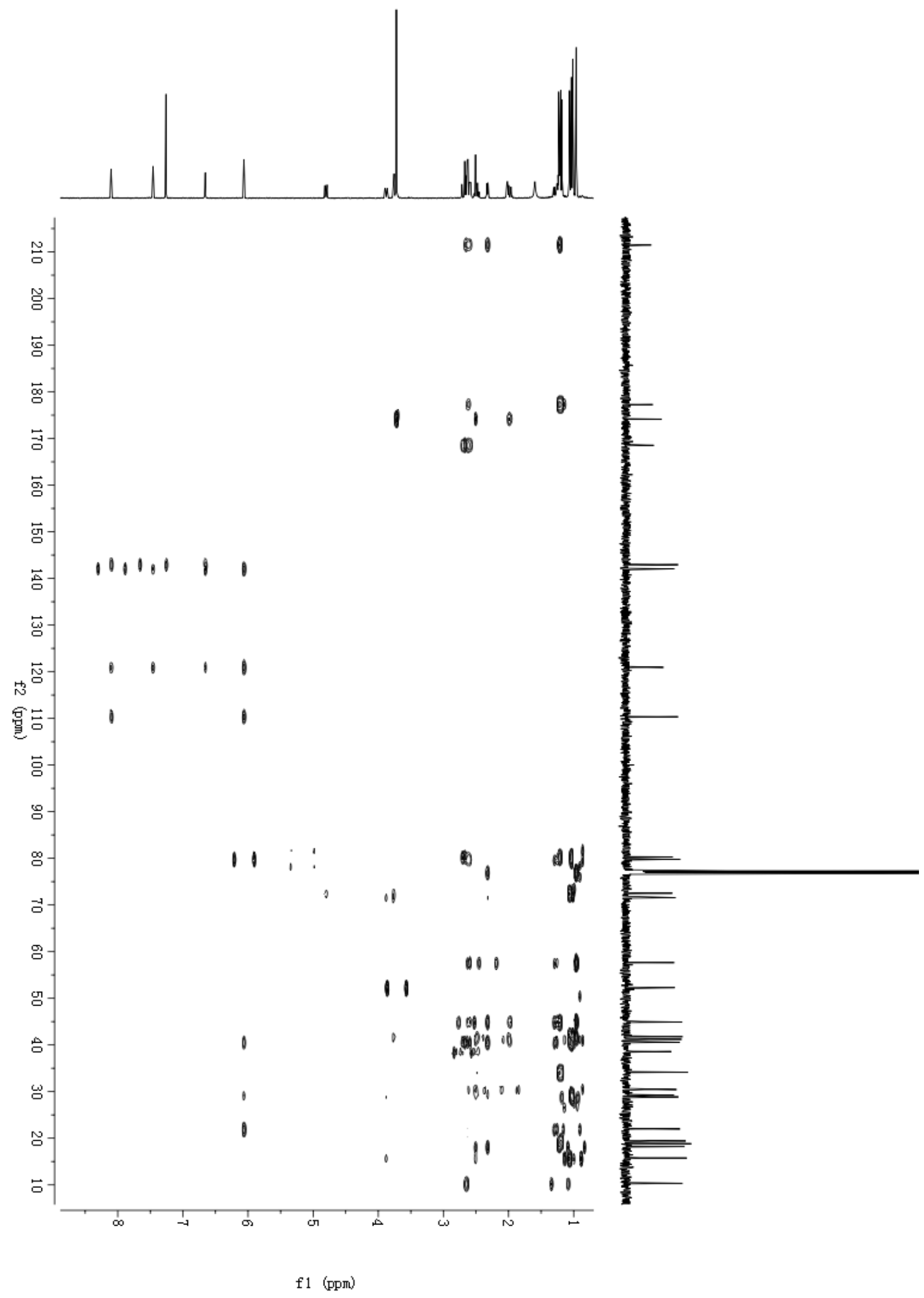


Figure S97. ESI(+)MS spectrum of 6-dehydroxycibacciferin F (12)

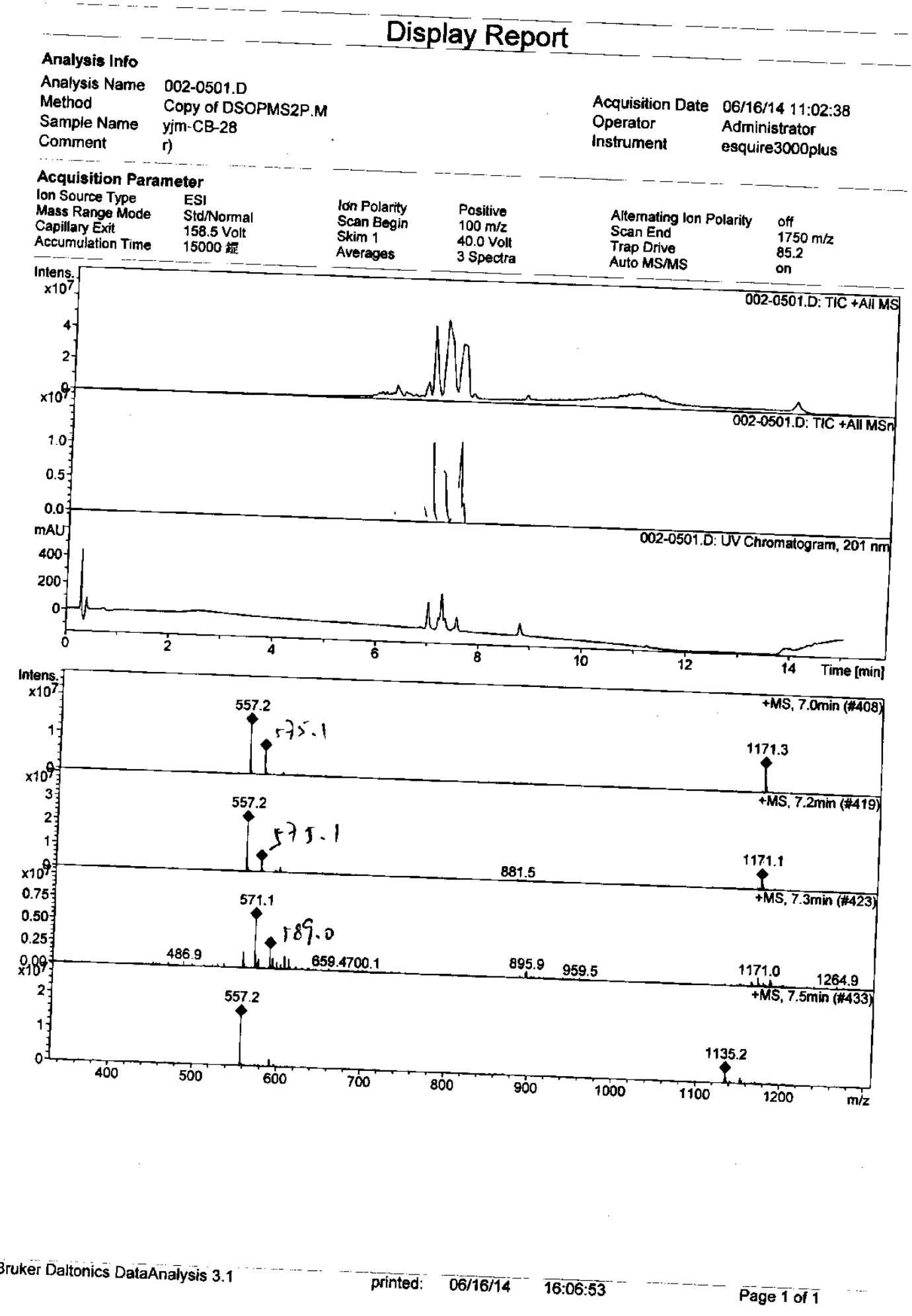


Figure S98. ESI(-)MS spectrum of 6-dehydroxycibacciferin F (12)

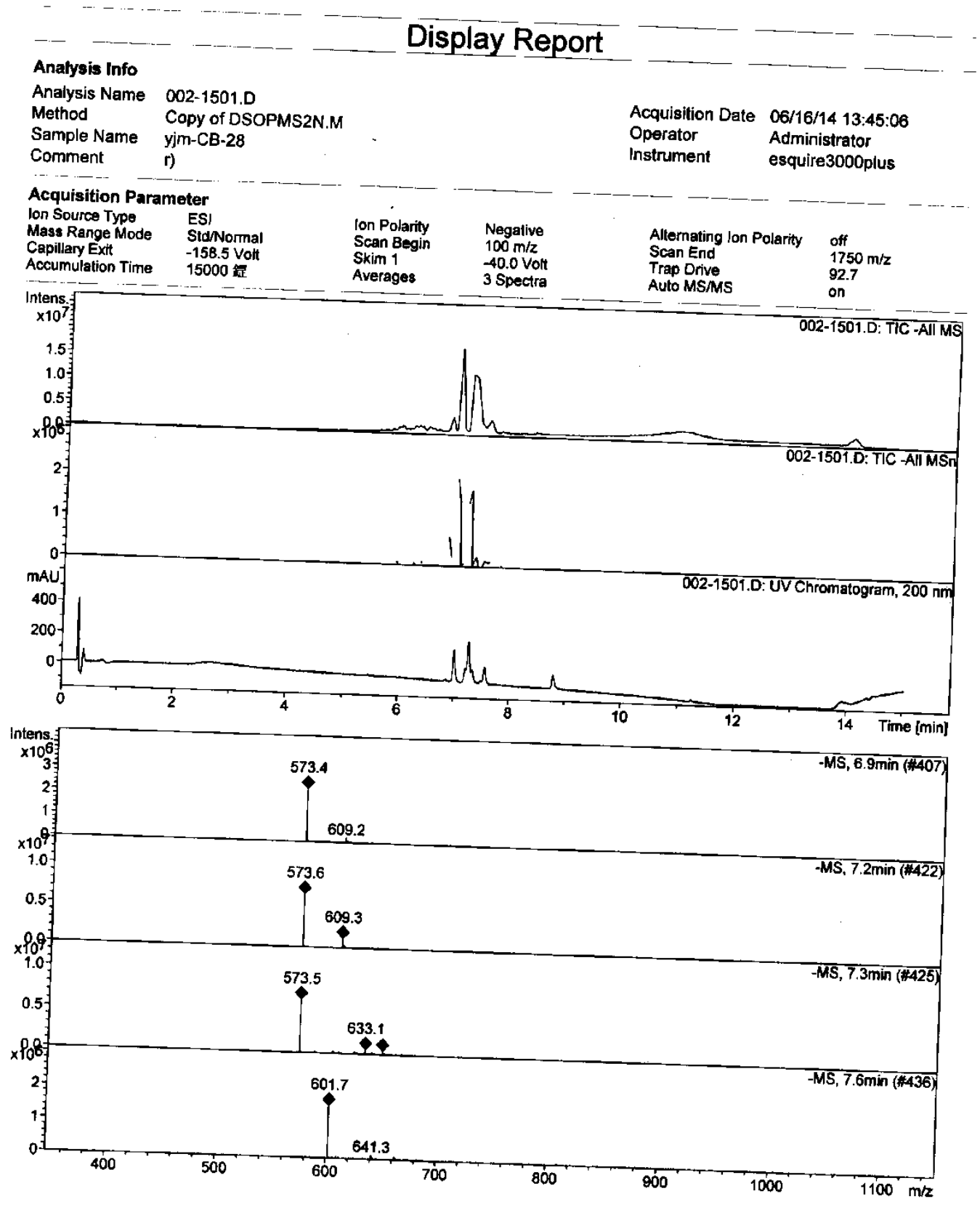


Figure S99. HRESI(+)MS spectrum of 6-dehydroxycibacciferin F (12)

Elemental Composition Report

Single Mass Analysis

Tolerance $=5.0$ PPM / DBE: $\min =-1.5, \max =50.0$

Element prediction: Off

Number of isotope peaks used for i-FIT $=3$

Monoisotopic Mass, Even Electron lons

242 formula(e) evaluated with 2 results within limits (up to 50 closest results for each mass)

Elements Used:

$\begin{array}{llll}\text { C: } 5-80 & \text { H: } 2-120 & \text { O: } 0-20 & \mathrm{Na}: 0-1\end{array}$

CB-28

LCT PXE KE324

05-Dec-2013

16:49:02

CB-28_1205 13 (0.284) AM2 (Ar, 10000.0,0.00,1.00); ABS; Cm (8:27)

1: TOF MS ES+

100

$\%$

592.3128

$0 \quad 582.3044 \quad 585.7650 \quad 587.2834589 .2914$

\begin{tabular}{l|l|l}
$593.3152 \quad 598.2703$ \\
\multicolumn{1}{|c|}{595.2693} & & 599.2707600 .2555
\end{tabular} 582.0 $84.0 \quad 586.0$

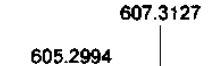

Minimum:

Maximum:

$5.0 \quad 5.0$

$-1.5$

Nass

Caic. Mass mDa PPM

DBE

597.2674

597.2676

$-0.2$

$-0.3$

10.5

i-FIT

i-FIT (Norm) Formula

597.2700

$-2.6 \quad-4.4$

$13.5-99.5$

0.0

C31 H42 010 Nâ

C33 H41 010 
Figure S100. IR spectrum of 6-dehydroxycibacciferin F (12)

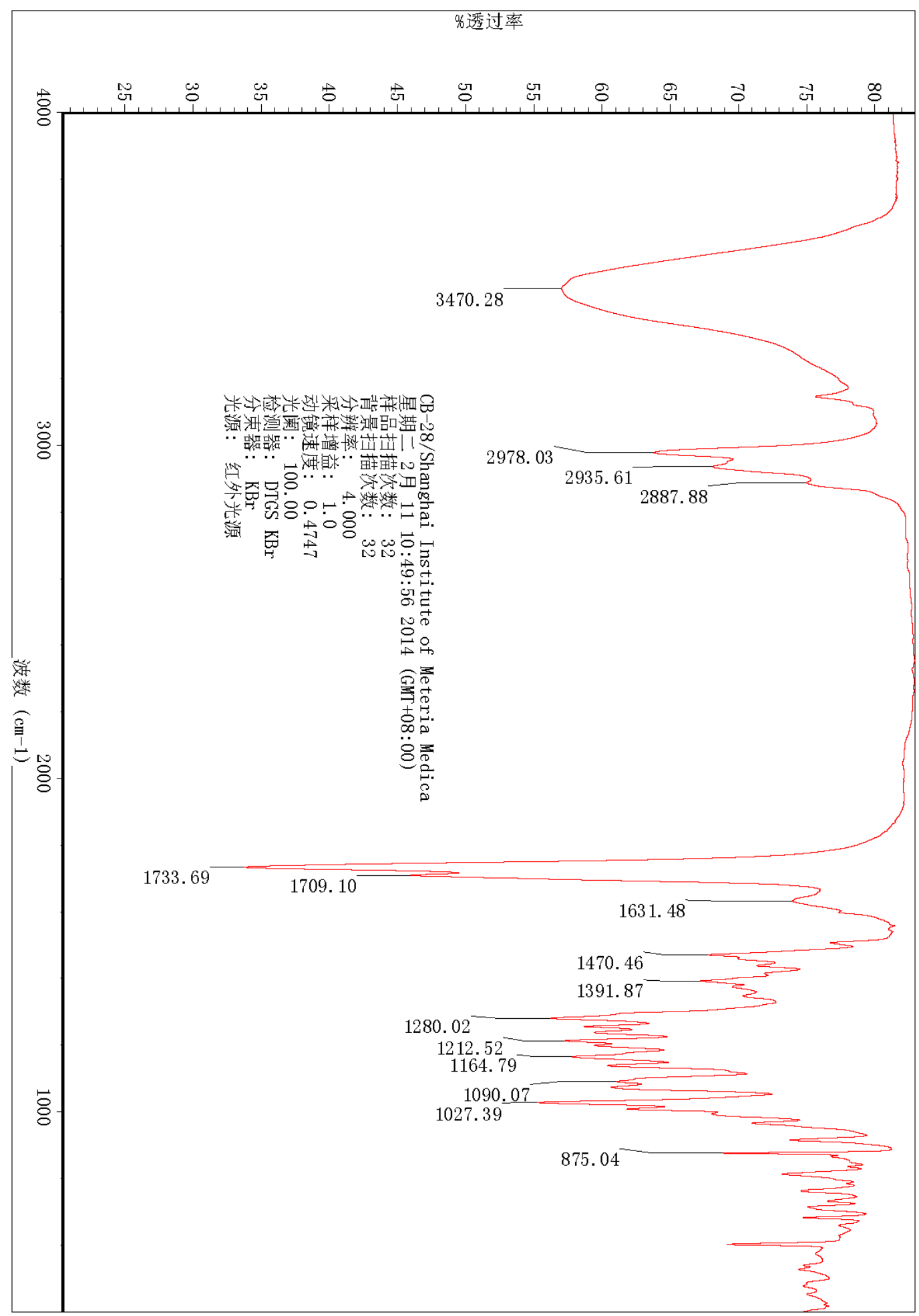


Figure S101. ${ }^{1} \mathrm{H}$ NMR spectrum of 12-deacetoxycibacciferin $\mathrm{E}(\mathbf{1 3})$ in $\mathrm{CDCl}_{3}$

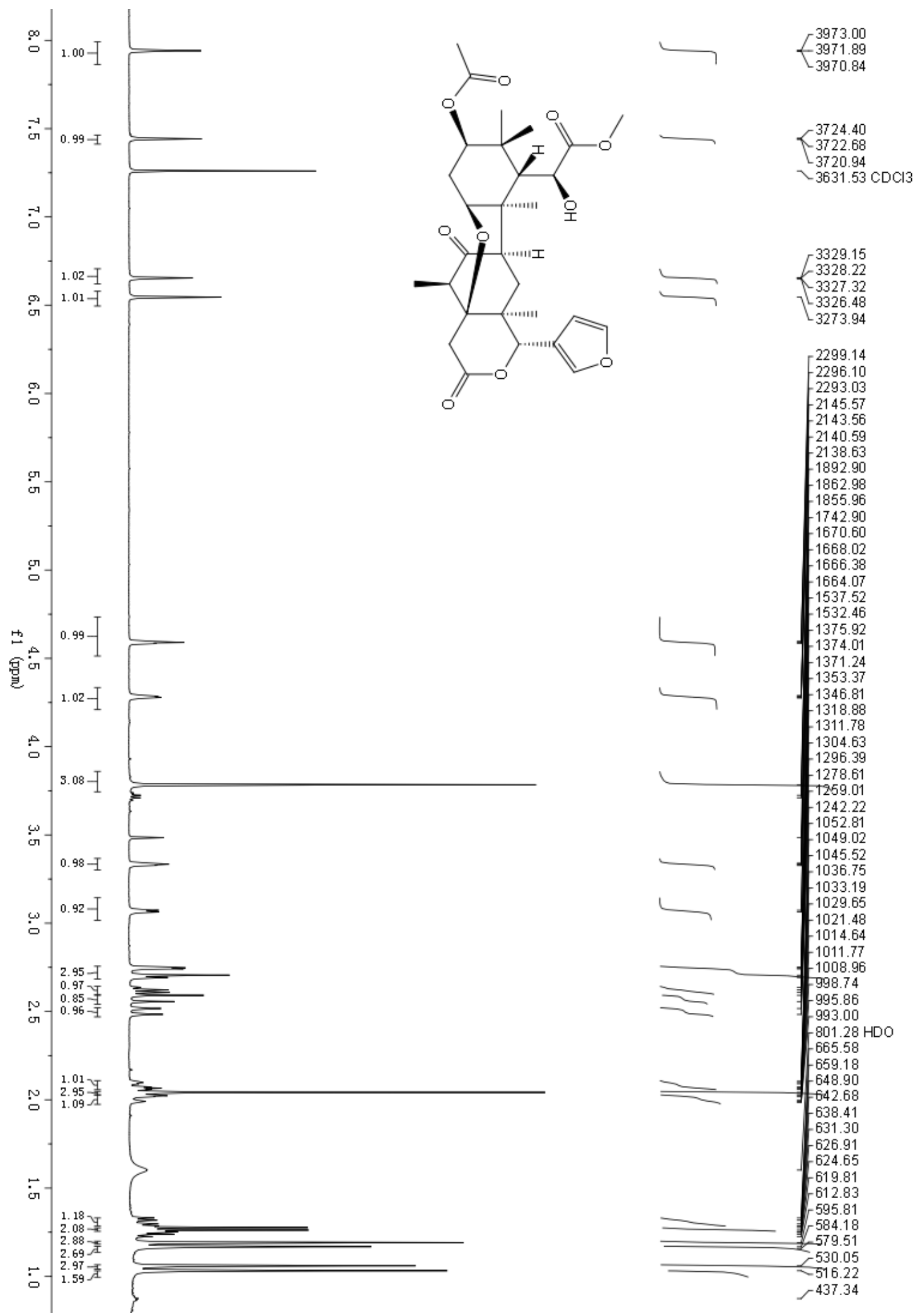


Figure S102. ${ }^{13} \mathrm{C}$ NMR spectrum of 12-deacetoxycibacciferin $\mathrm{E}(\mathbf{1 3})$ in $\mathrm{CDCl}_{3}$

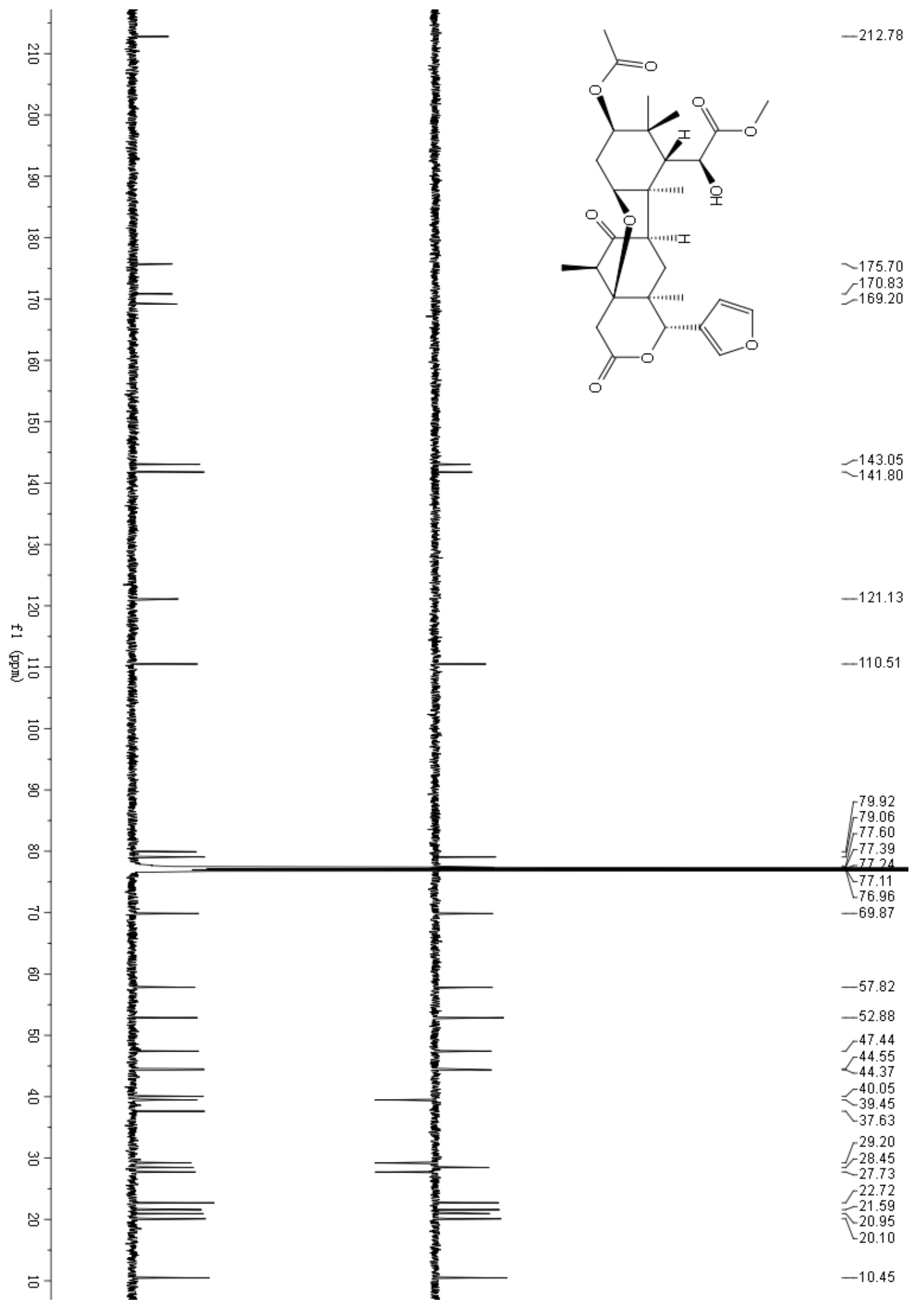


Figure S103. HSQC spectrum of 12-deacetoxycibacciferin E (13) in $\mathrm{CDCl}_{3}$

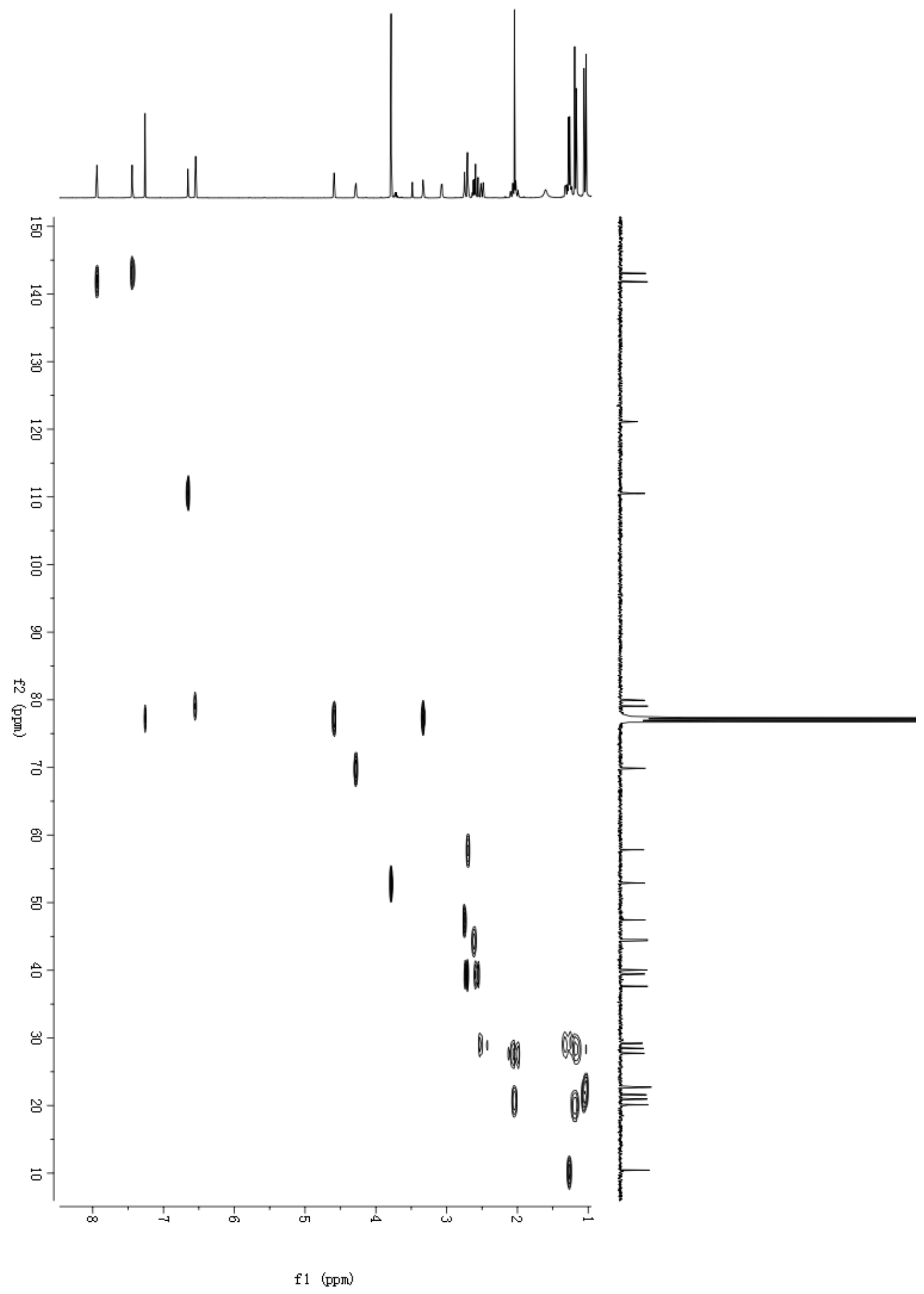


Figure S104. HMBC spectrum of 12-deacetoxycibacciferin E (13) in $\mathrm{CDCl}_{3}$

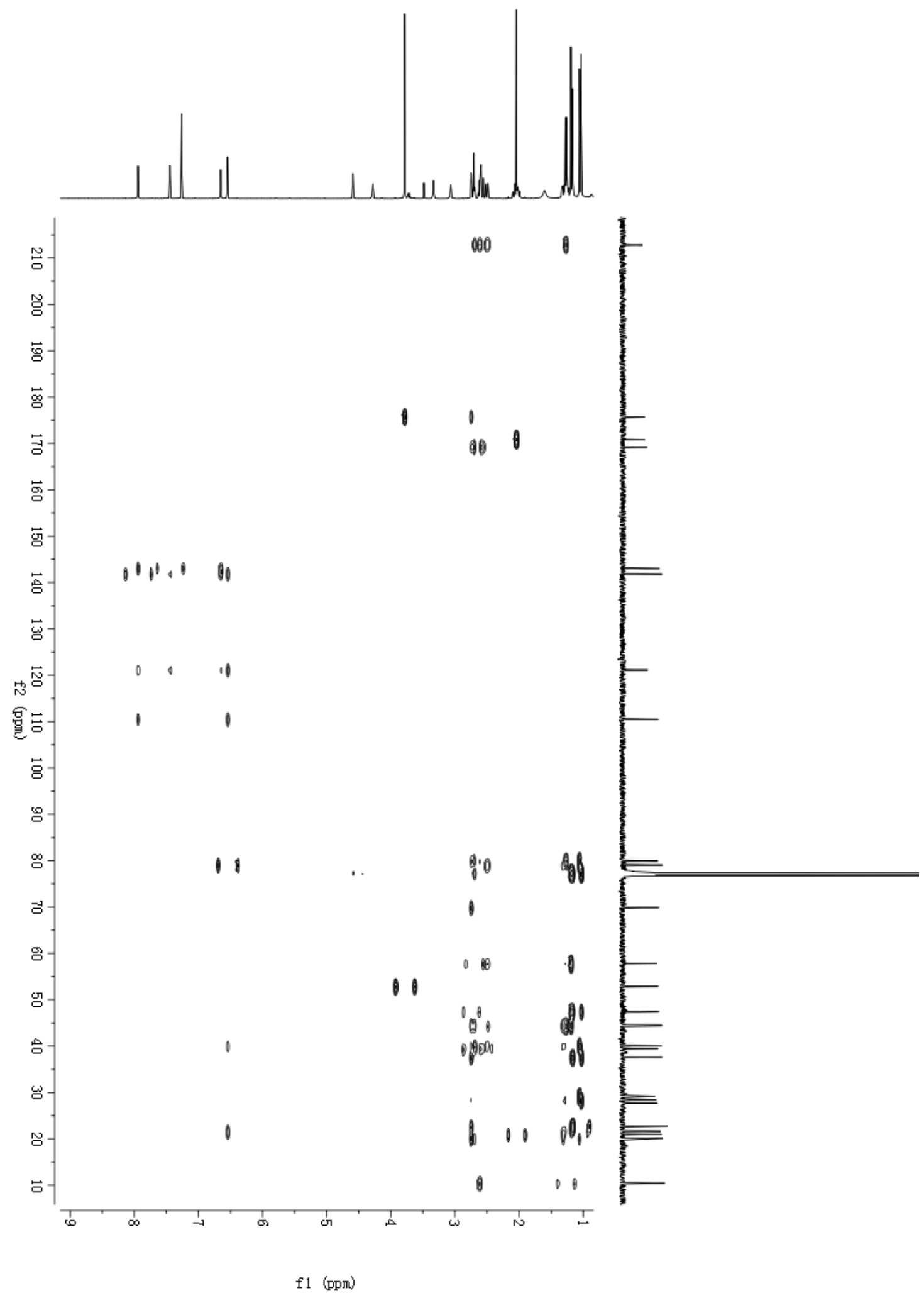


Figure S105. ESI(+)MS spectrum of 12-deacetoxycibacciferin E (13)

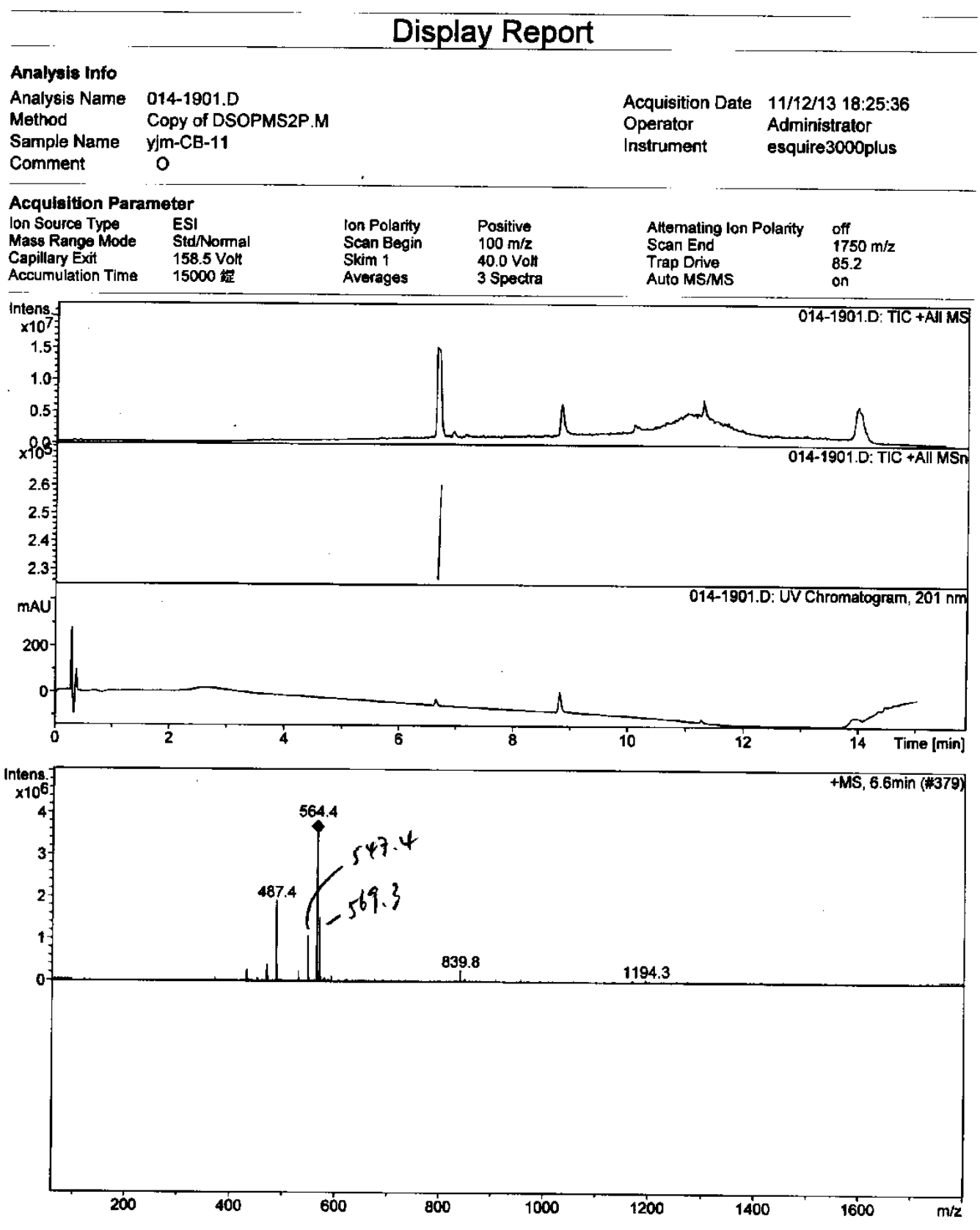

Bruker Daltonics DataAnalysis $3.1 \quad$ printed: $11 / 13 \overline{13} \quad 09: \overline{5}: \overline{13}-11$ Page 1 
Figure S106. ESI(-)MS spectrum of 12-deacetoxycibacciferin E (13)

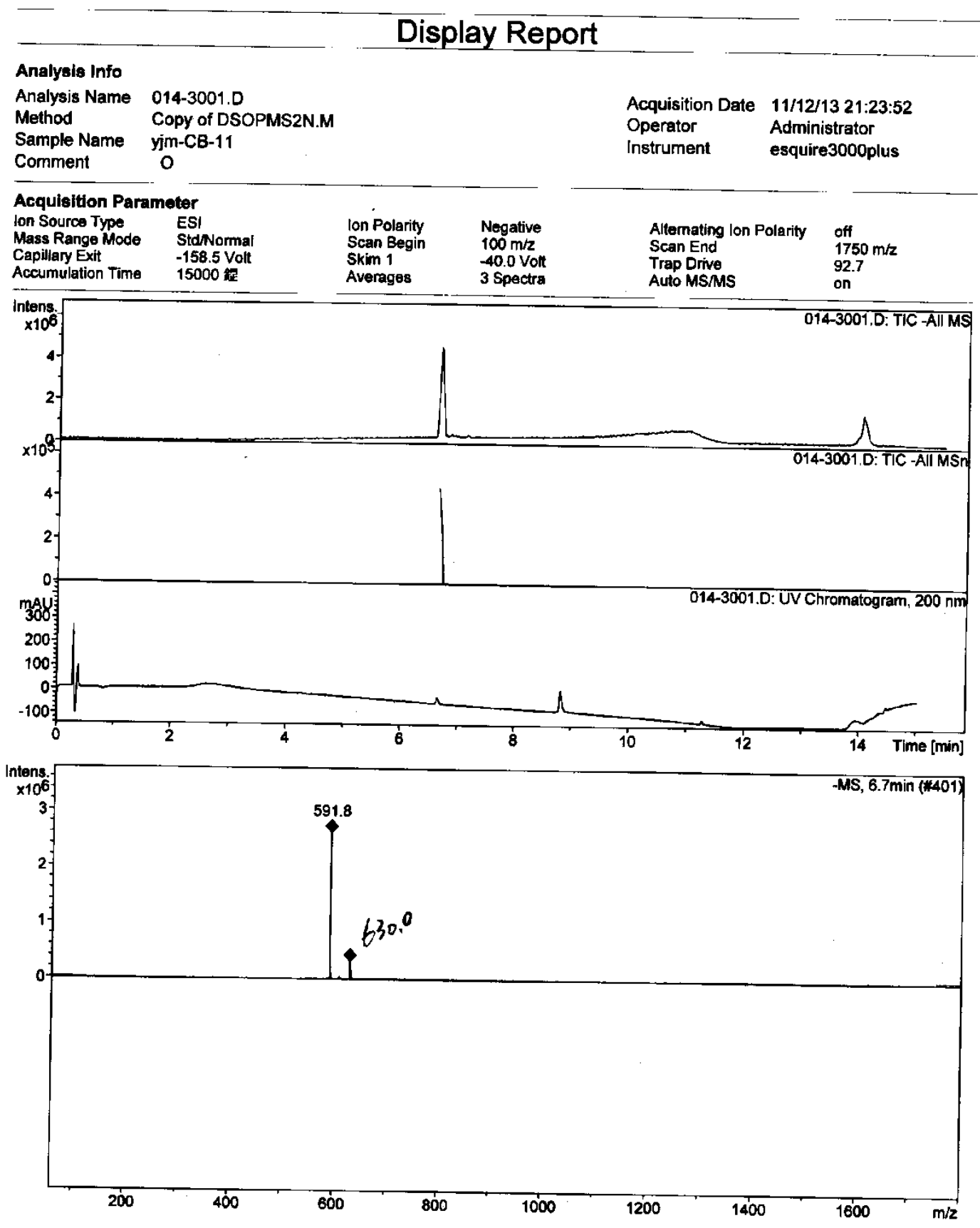


Figure S107. HRESI(+)MS spectrum of 12-deacetoxycibacciferin E (13)

\section{Elemental Composition Report}

Single Mass Analysis

Tolerance $=3.0 \mathrm{PPM} /$ I DBE: $\min =-1.5, \max =50.0$
Element prediction: Off

Number of isotope peaks used for i-FIT $=3$

Monoisotopic Mass, Even Electron lons

228 formula(e) evaluated with 1 results within limits (up to 50 closest results for each mass)

Elements Used:

$\begin{array}{llll}\text { C: } 5-80 & \text { H: } 2-120 & \text { O: } 0-20 \quad \mathrm{Na}: 0-1 \\ \text { CB-11 } & \end{array}$

LCT PXE KE324

CB-11_1119 26 (0.566) AM2 (Ar, 10000.0,0.00,1.00); ABS; Cm (25:41)

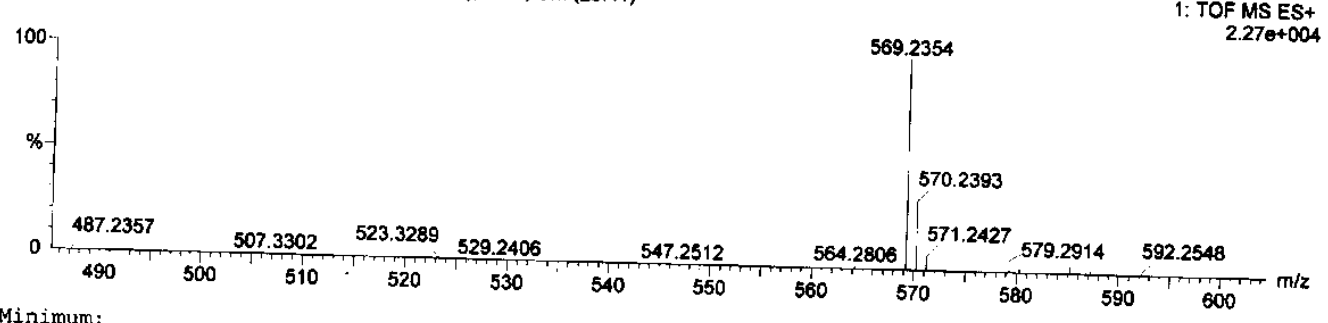

Minimum:

Maximum:

-1.5
50.0

Mass Calc. Mass mDa PPM DBE

$569.2354 \quad 569.2363 \quad-0.9$

I-FIT i-FIT (Norm) Formula

$\begin{array}{llllllll}569.2363 & -0.9 & -1.6 & 10.5 & 69.4 & 0.0 & \text { C29 H38 } 010 \mathrm{~N}\end{array}$


Figure S108. IR spectrum of 12-deacetoxycibacciferin E (13)

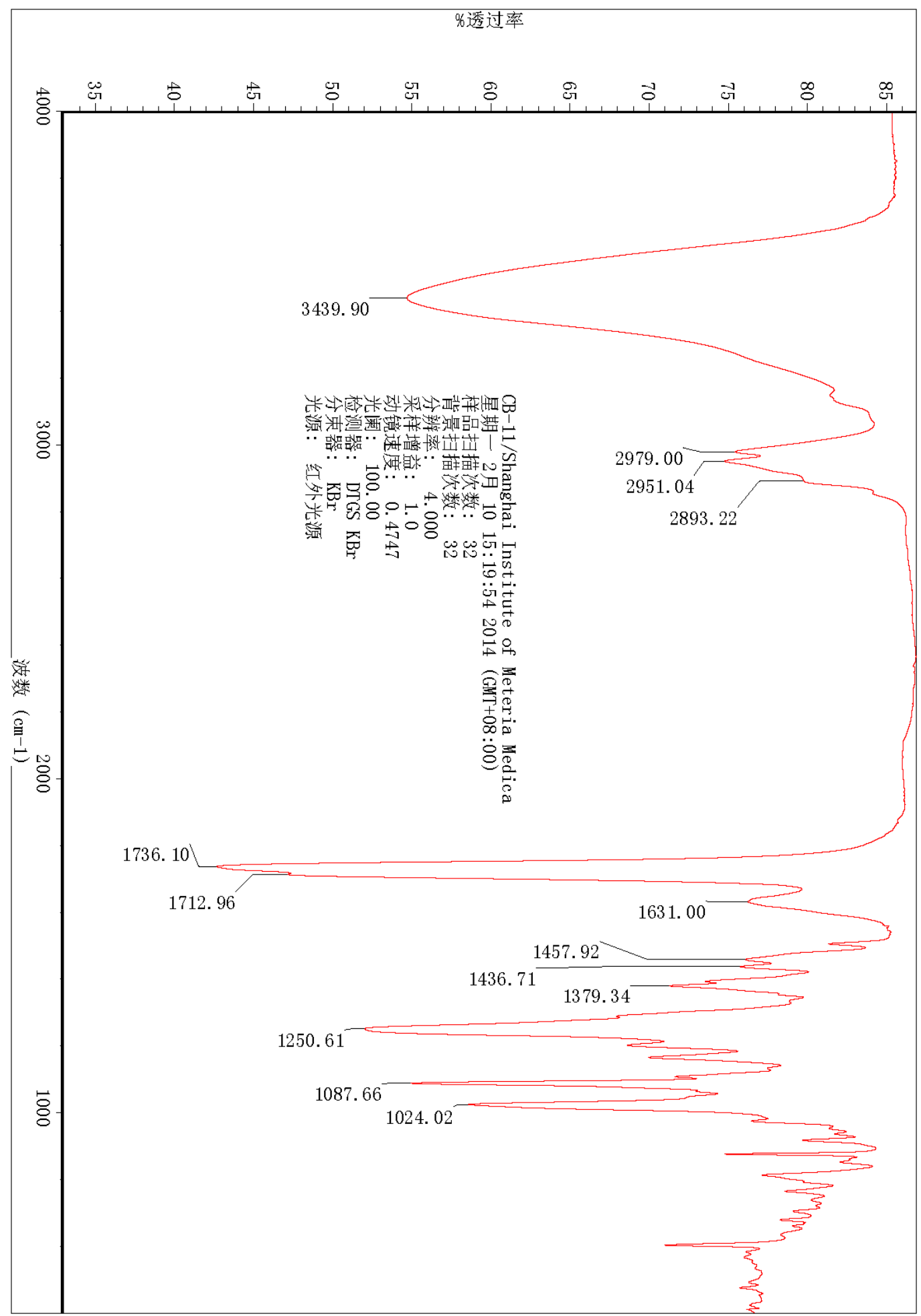


Figure S109. ${ }^{1} \mathrm{H}$ NMR spectrum of $2 \beta$-acetoxy-12-deacetoxycibacciferin E (14) in $\mathrm{CDCl}_{3}$

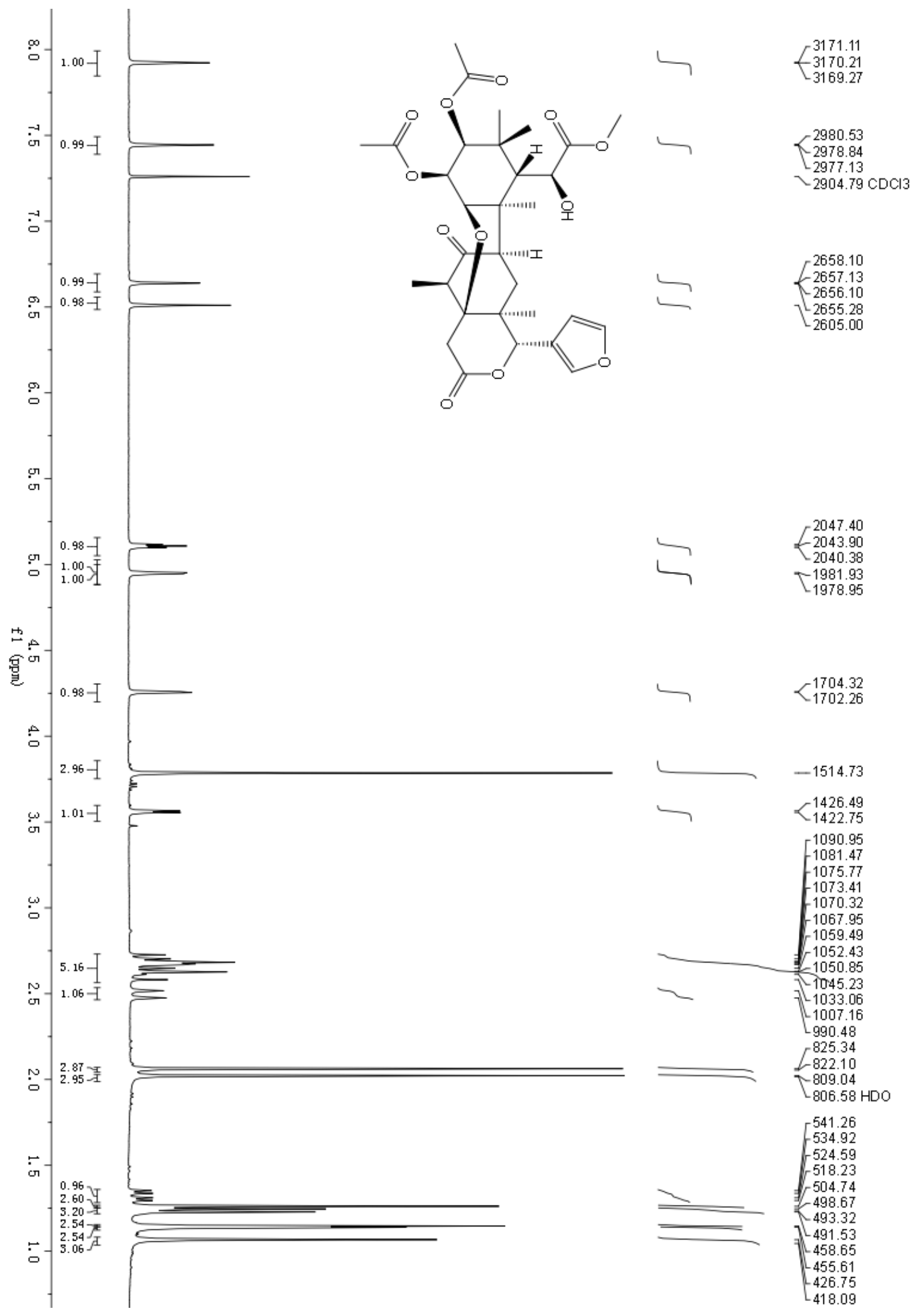


Figure S110. ${ }^{13} \mathrm{C}$ NMR spectrum of $2 \beta$-acetoxy-12-deacetoxycibacciferin E (14) in $\mathrm{CDCl}_{3}$

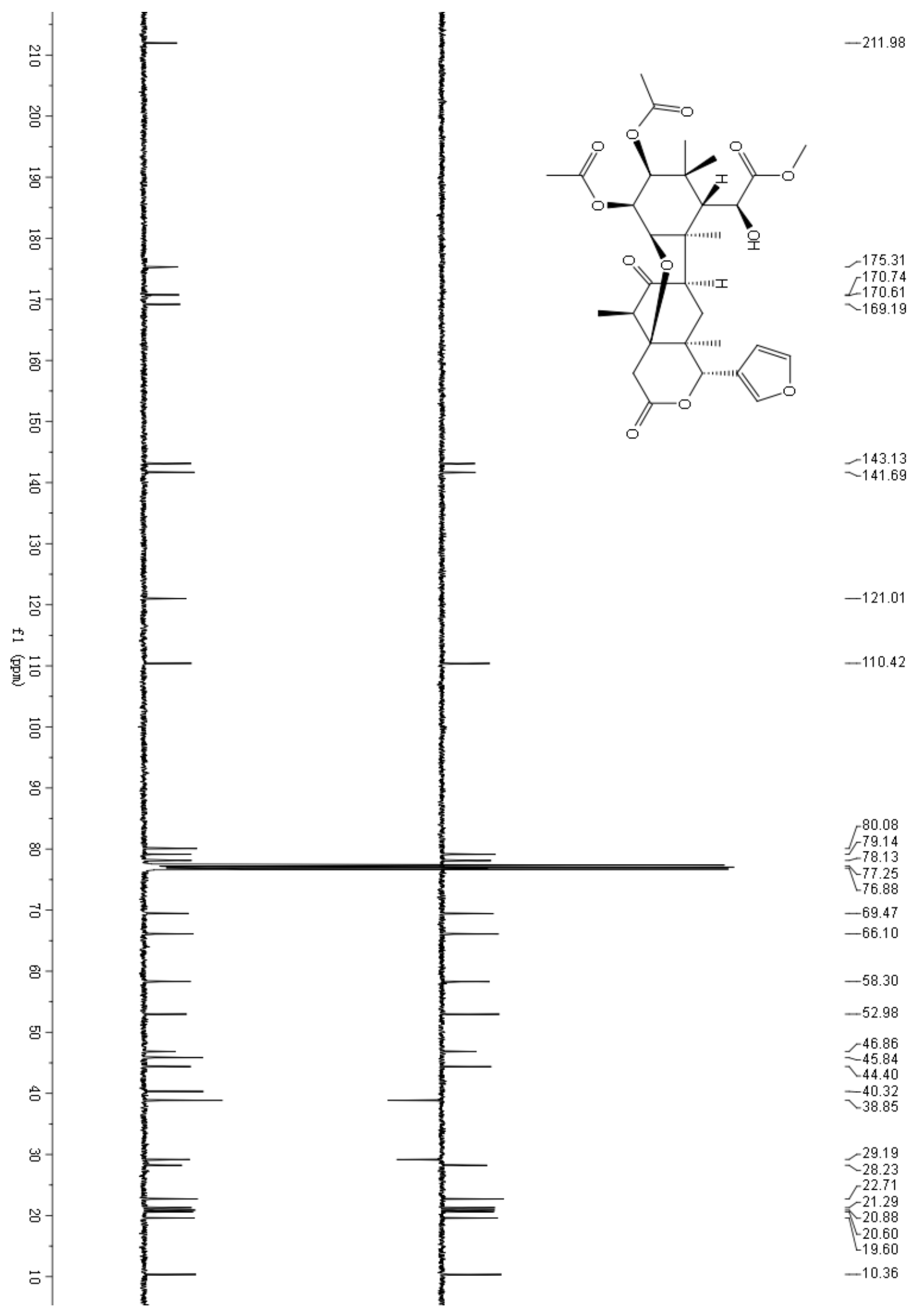


Figure S111. HSQC spectrum of $2 \beta$-acetoxy-12-deacetoxycibacciferin E (14) in $\mathrm{CDCl}_{3}$

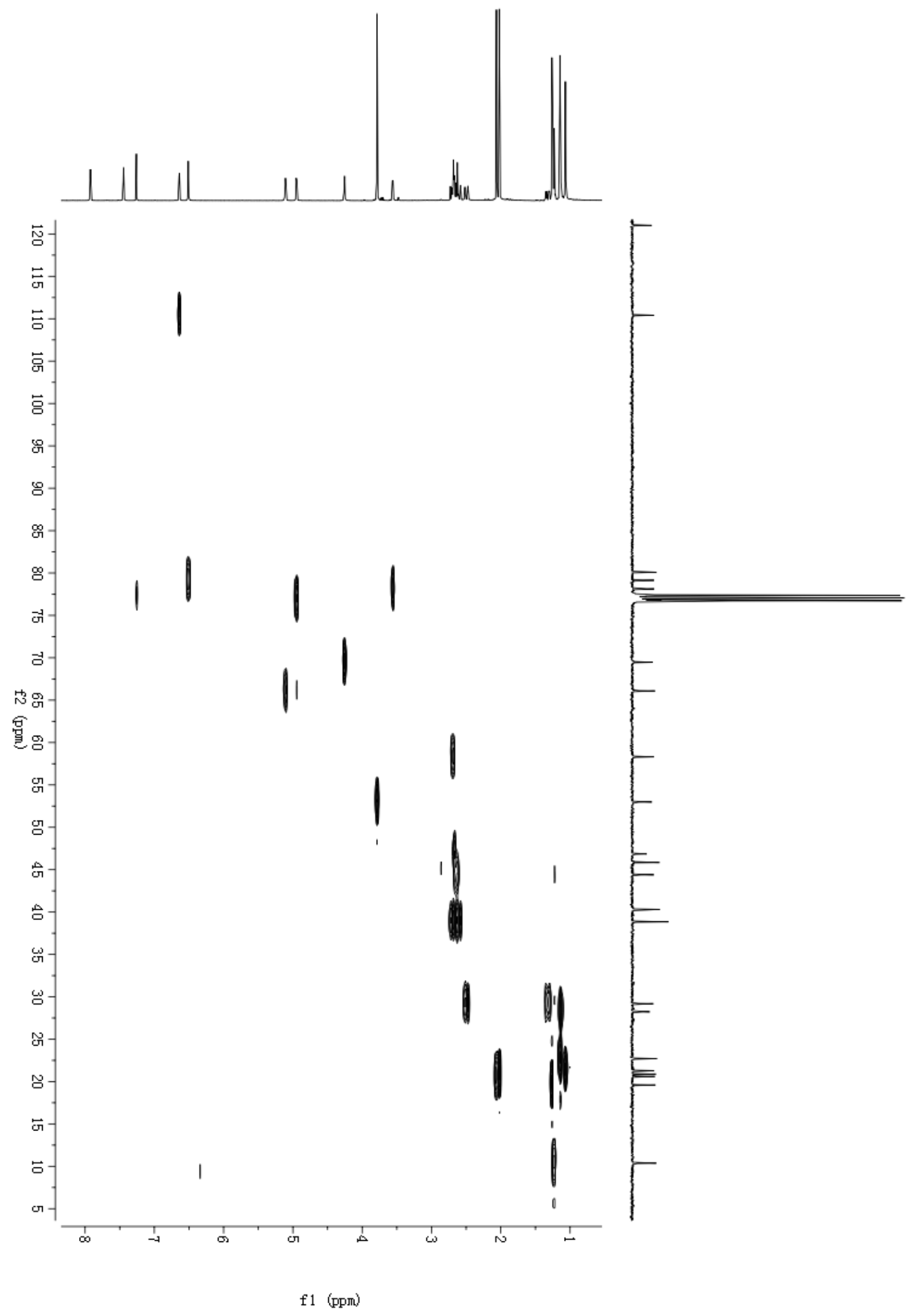


Figure S112. HMBC spectrum of $2 \beta$-acetoxy-12-deacetoxycibacciferin E (14) in $\mathrm{CDCl}_{3}$

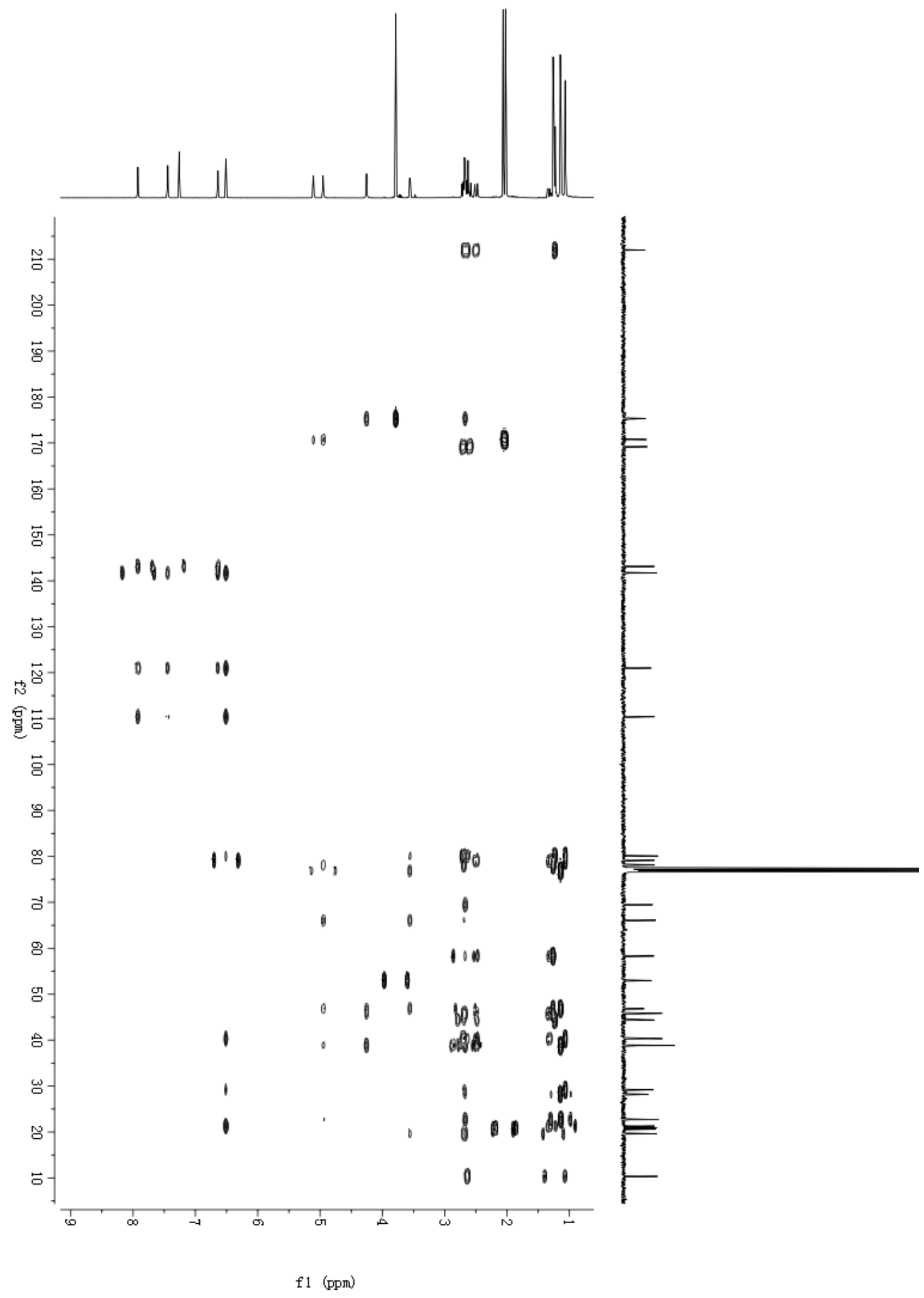


Figure S113. ESI(+)MS spectrum of $2 \beta$-acetoxy-12-deacetoxycibacciferin E (14)

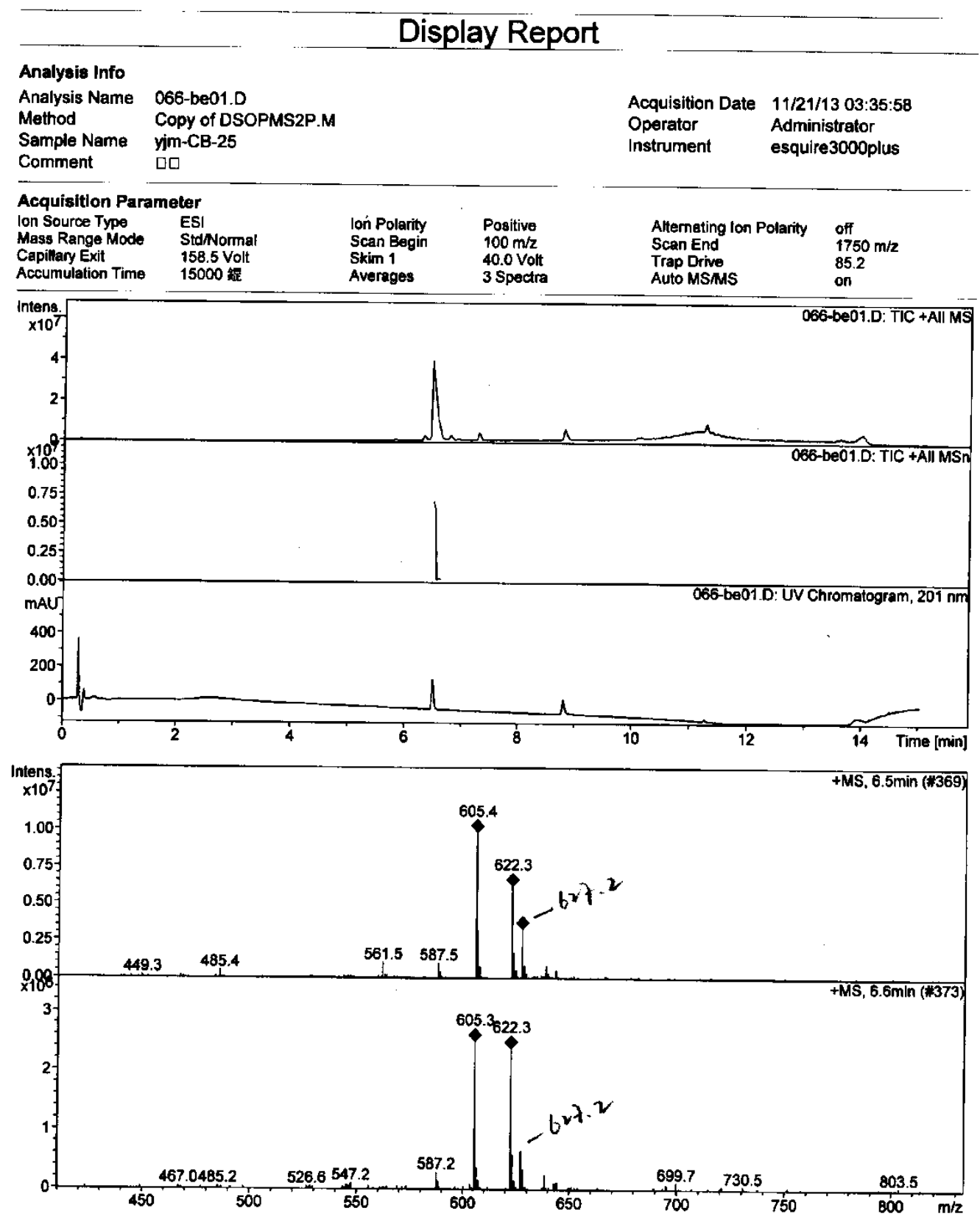


Figure S114. ESI(-)MS spectrum of $2 \beta$-acetoxy-12-deacetoxycibacciferin E (14)

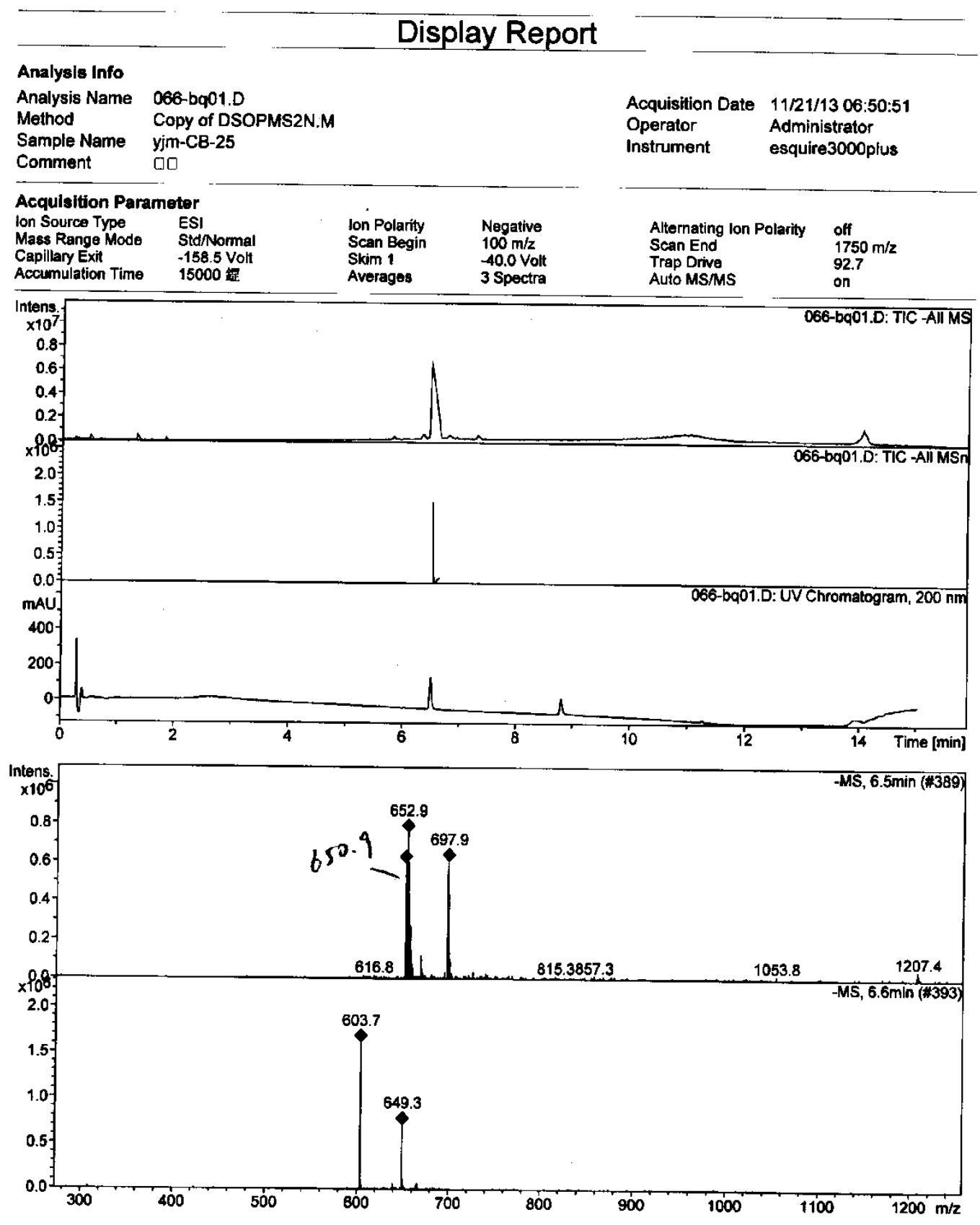


Figure S115. HRESI(+)MS spectrum of $2 \beta$-acetoxy-12-deacetoxycibacciferin E (14)

Elemental Composition Report

Page 1

Single Mass Analysis

Tolerance $=3.0 \mathrm{PPM} /$ DBE: $\min =-1.5, \max =50.0$

Element prediction: Off

Number of isotope peaks used for $\mathrm{i}-\mathrm{FIT}=3$

Monoisotopic Mass, Even Electron lons

257 formula(e) evaluated with 1 results within limits (up to 50 closest results for each mass)

Elements Used:

$\begin{array}{llll}\text { C: } 5-80 & \text { H: } 2-120 & \text { O: } 0-20 & \mathrm{Na}: 0-1\end{array}$

CB-25 ' LCT PXE KE324

18-Jul-2014

CB-25_20140718 33 (0.725) AM2 (Ar, 10000.0,0.00,1.00); ABS; Cm (33:41)

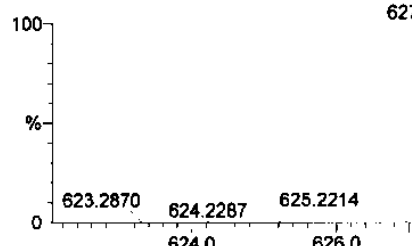

627.2422

Minimum: 624.0

$629.3854 \quad 630.3875$

\subsection{3}

$5.0 \quad 3.0 \quad 50.0$

Mass Calc. Mass mDa PPM DBE i-FIT i-FIT \{Norn\} Formula

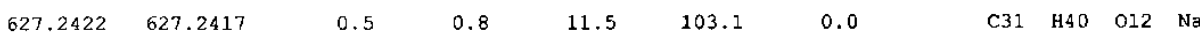


Figure S116. IR spectrum of $2 \beta$-acetoxy-12-deacetoxycibacciferin E (14)

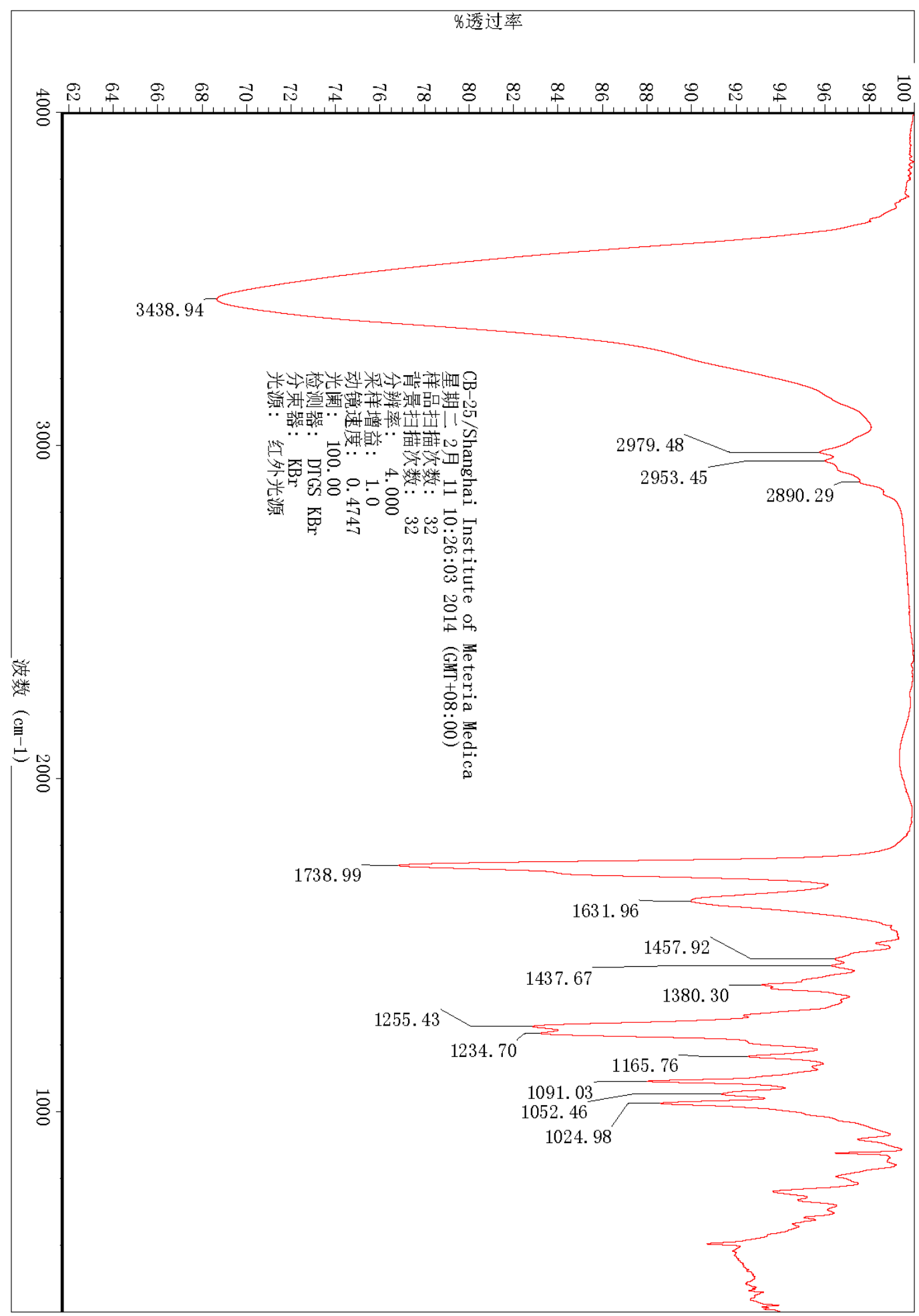


Figure S117. ${ }^{1} \mathrm{H}$ NMR spectrum of cibacciferin $\mathrm{G}(\mathbf{1 5})$ in $\mathrm{CDCl}_{3}$

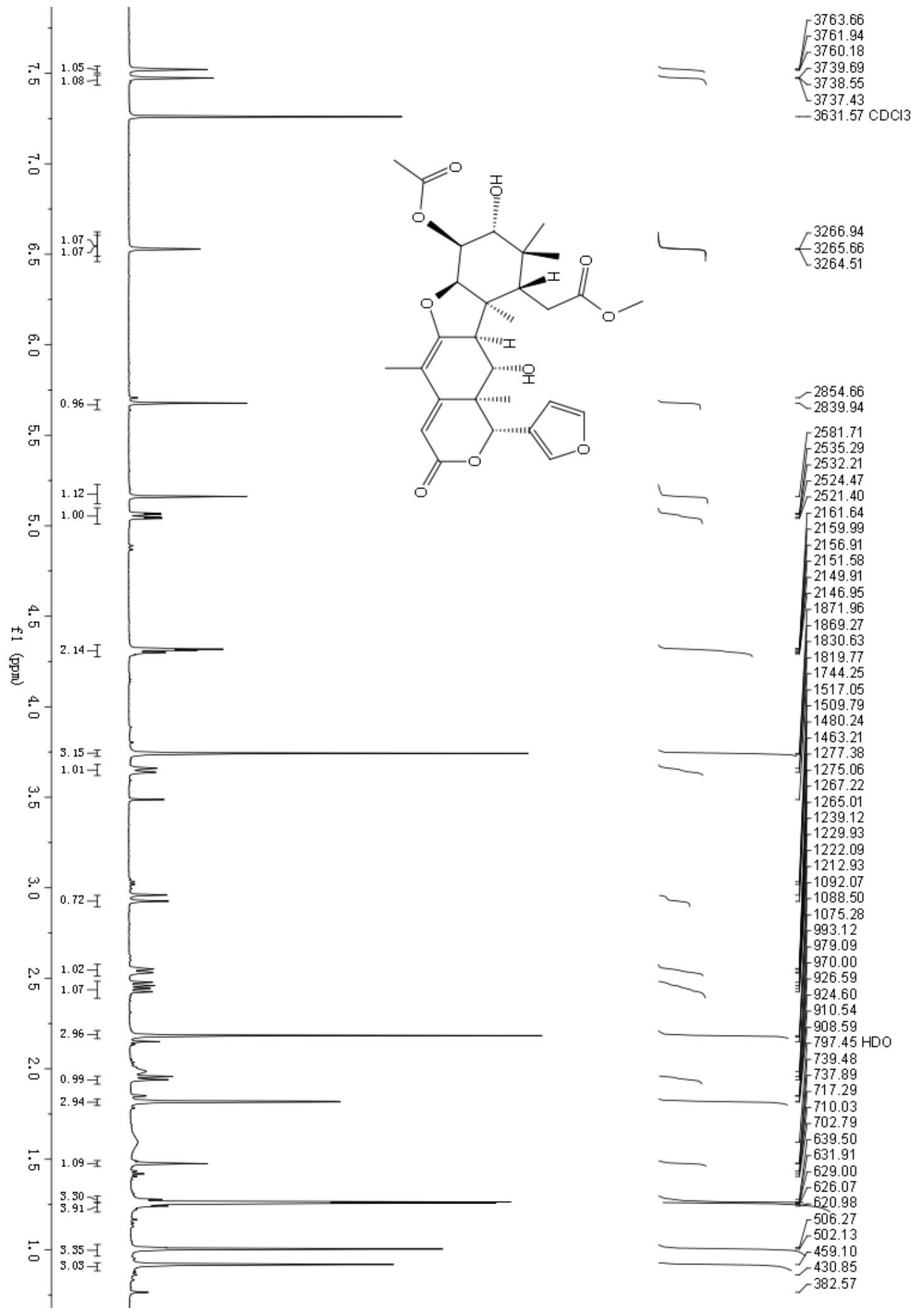


Figure S118. ${ }^{13} \mathrm{C}$ NMR spectrum of cibacciferin $\mathrm{G}(\mathbf{1 5})$ in $\mathrm{CDCl}_{3}$

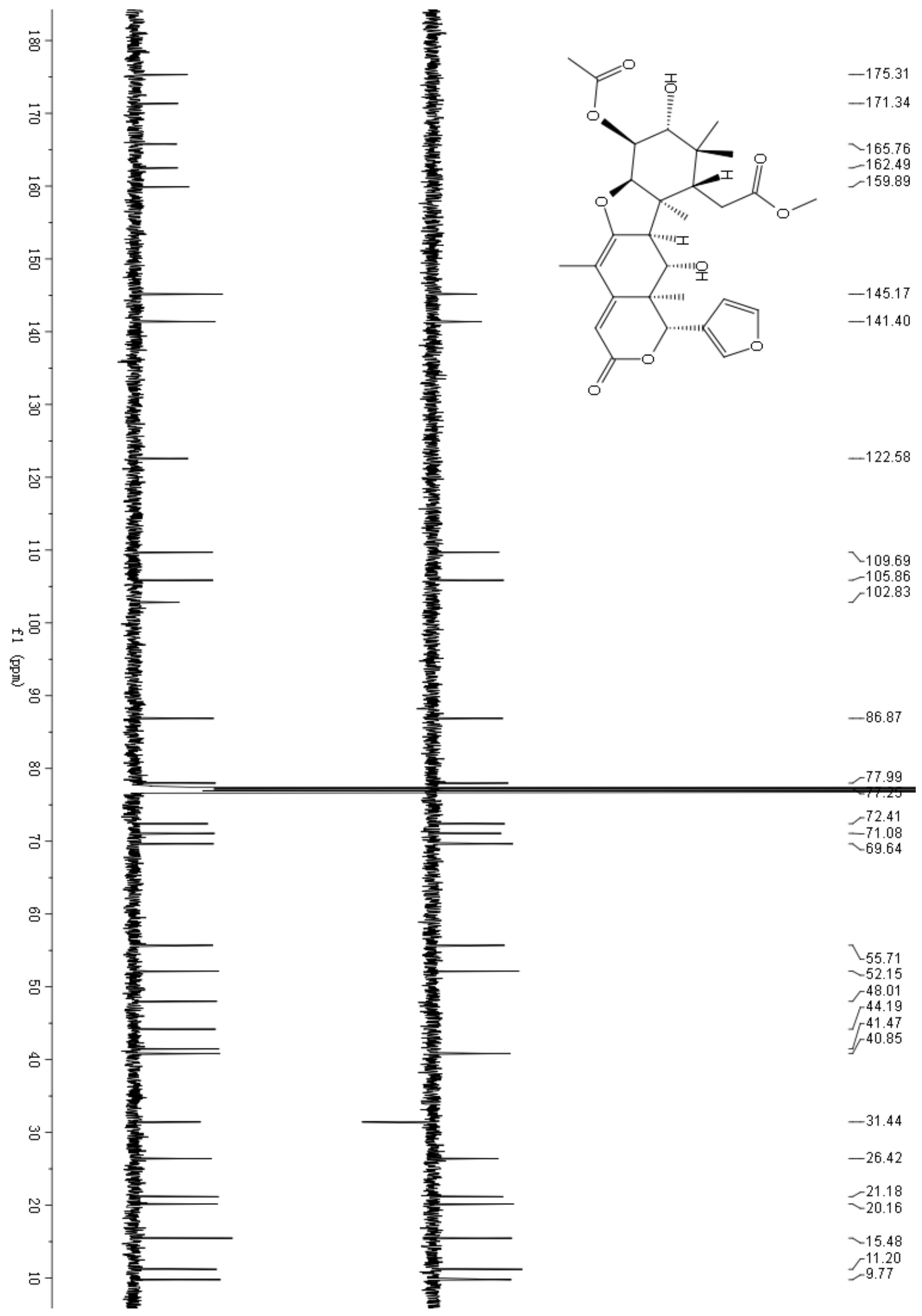


Figure S119. HSQC spectrum of cibacciferin $\mathrm{G}(\mathbf{1 5})$ in $\mathrm{CDCl}_{3}$

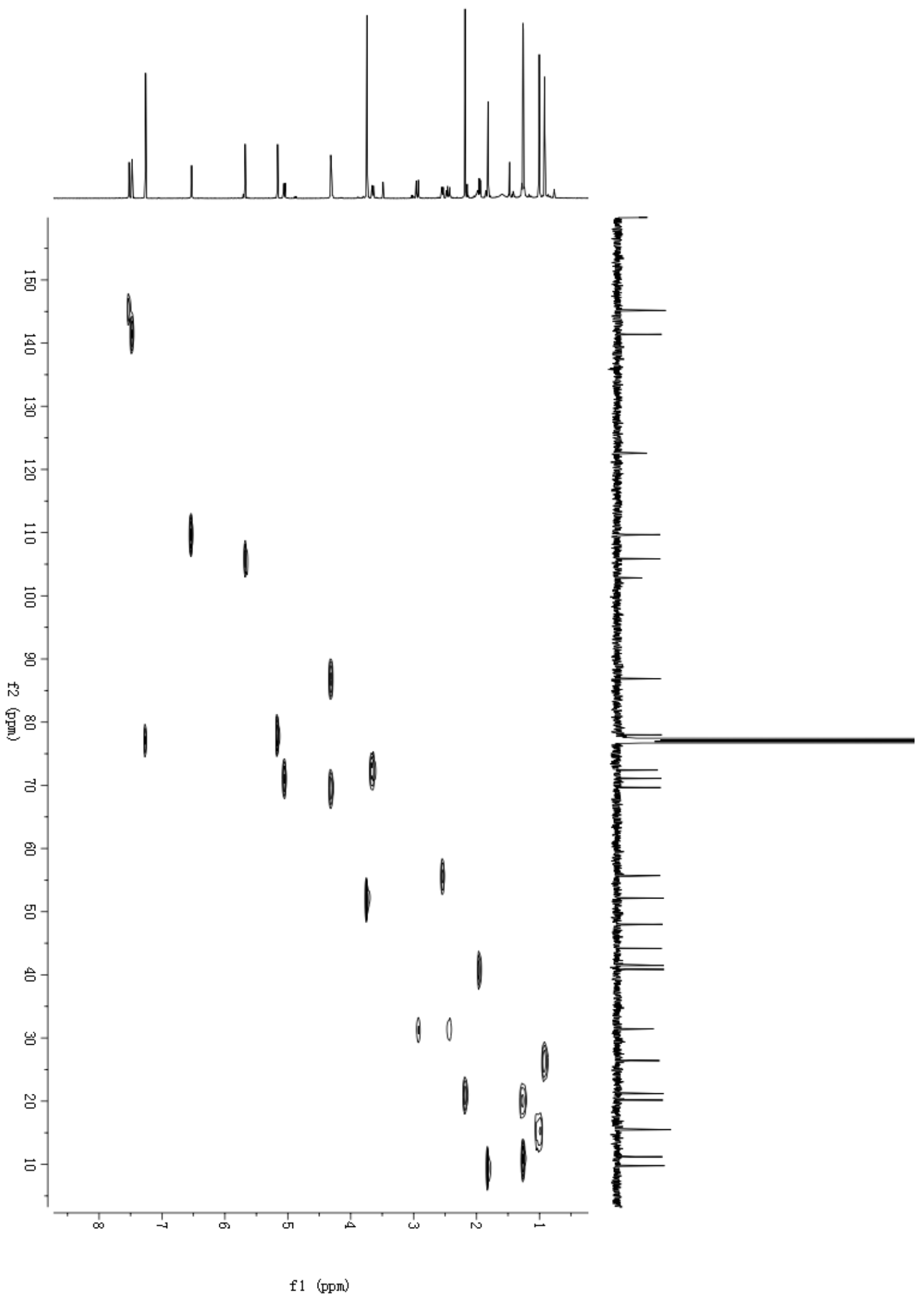

129 
Figure S120. $\mathrm{HMBC}$ spectrum of cibacciferin $\mathrm{G}(\mathbf{1 5})$ in $\mathrm{CDCl}_{3}$

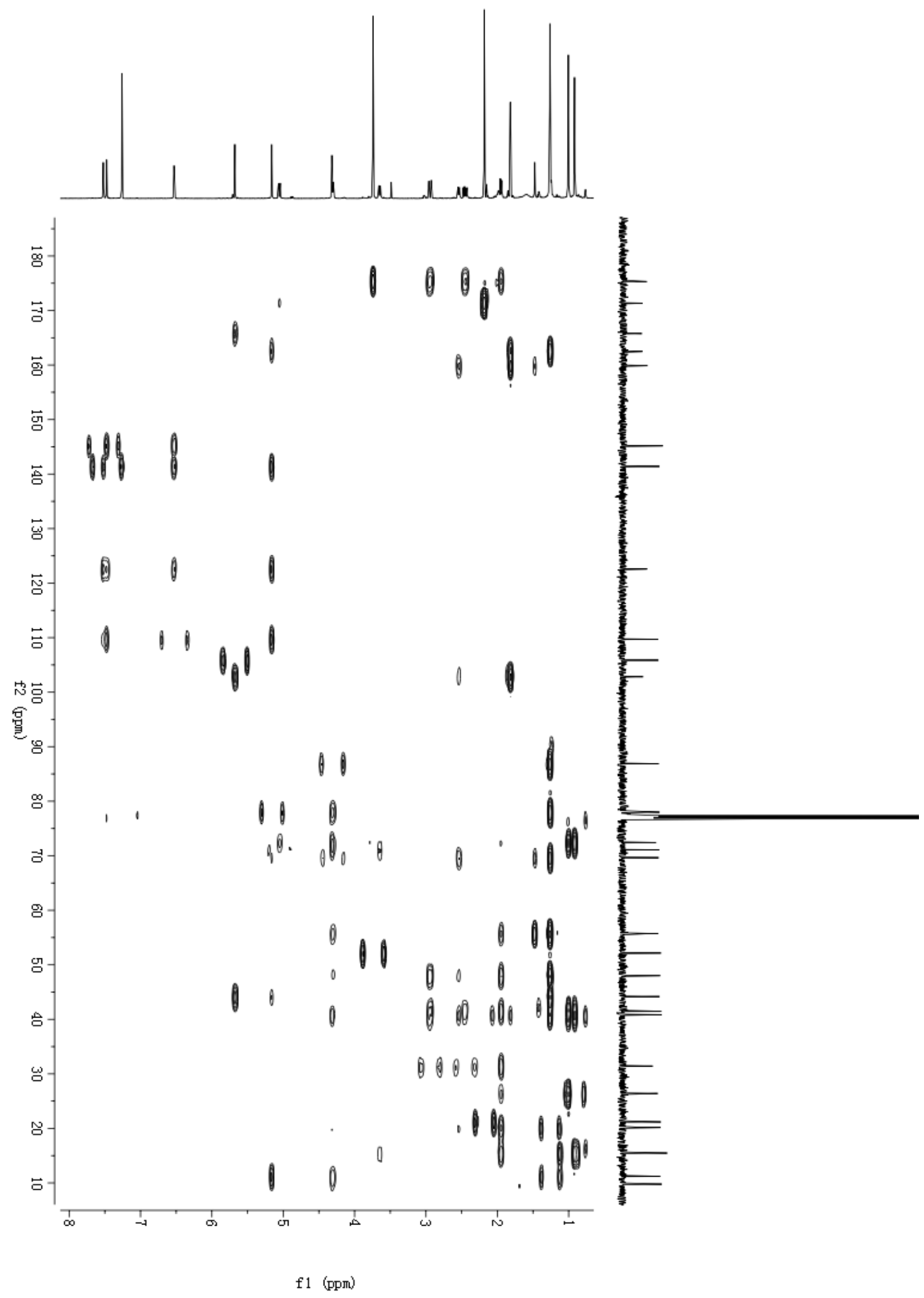


Figure S121. ROESY spectrum of cibacciferin $\mathrm{G}(15)$ in $\mathrm{CDCl}_{3}$

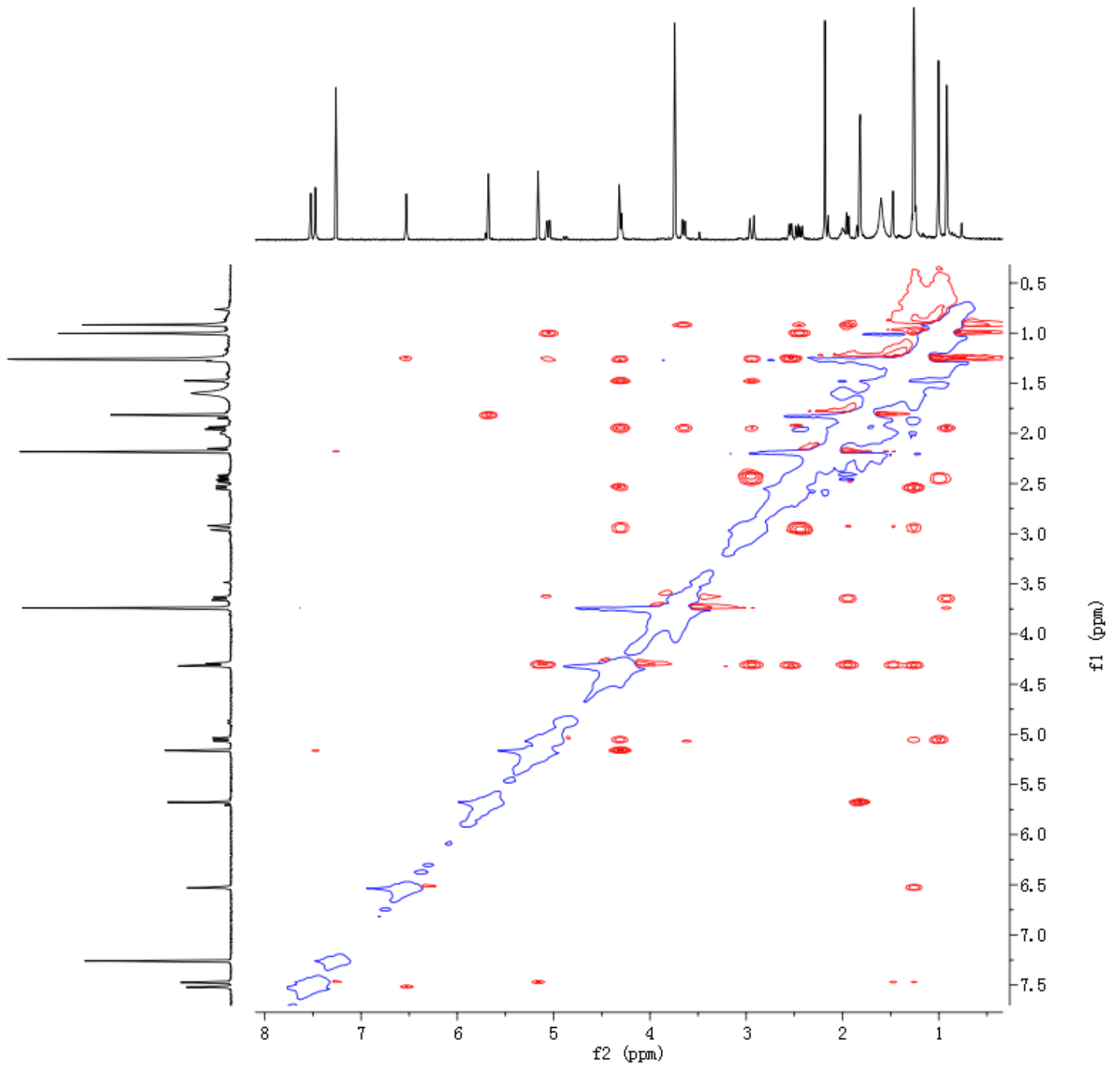


Figure S122. ESI(+)MS spectrum of cibacciferin G (15)

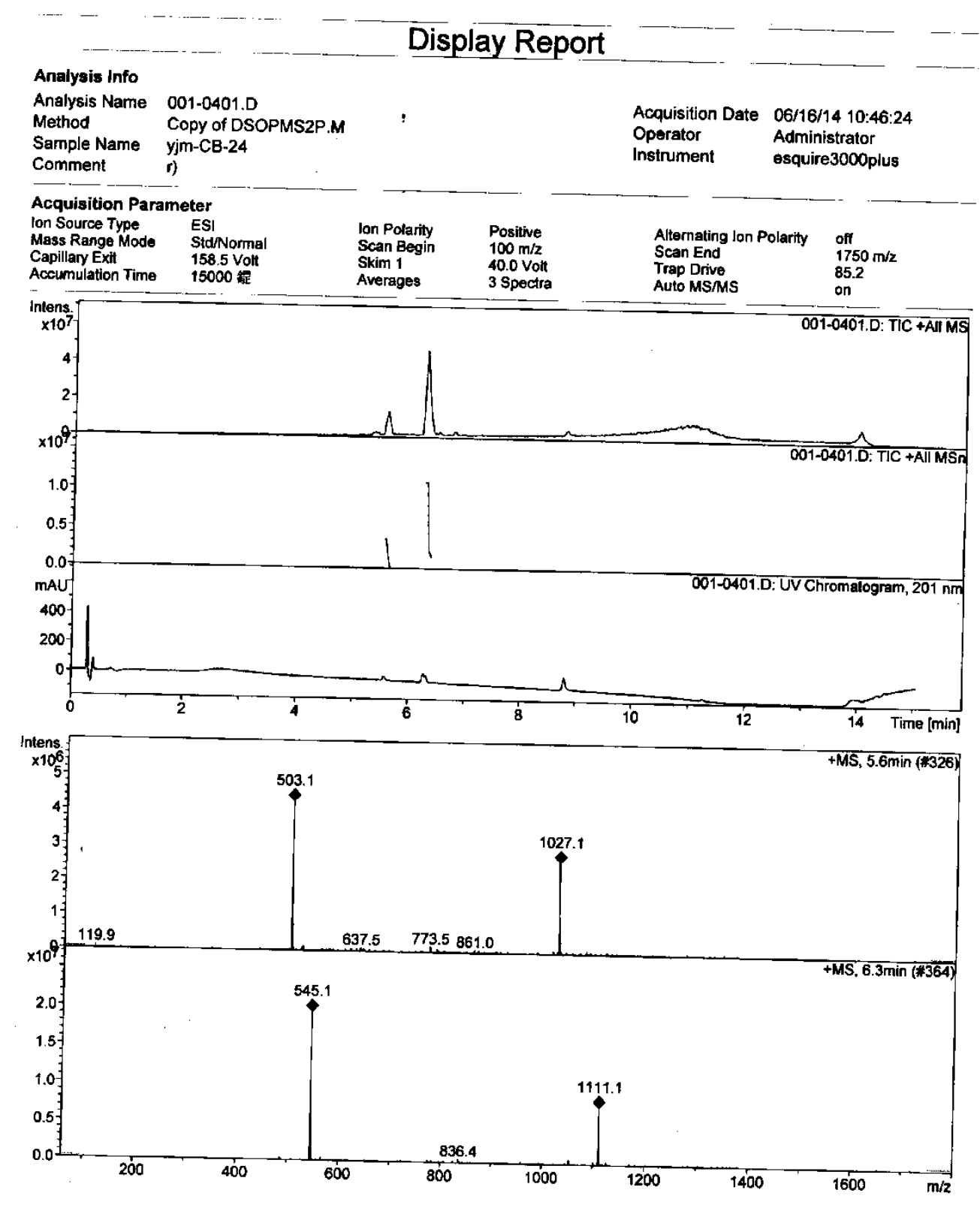


Figure S123. ESI(-)MS spectrum of cibacciferin G (15)

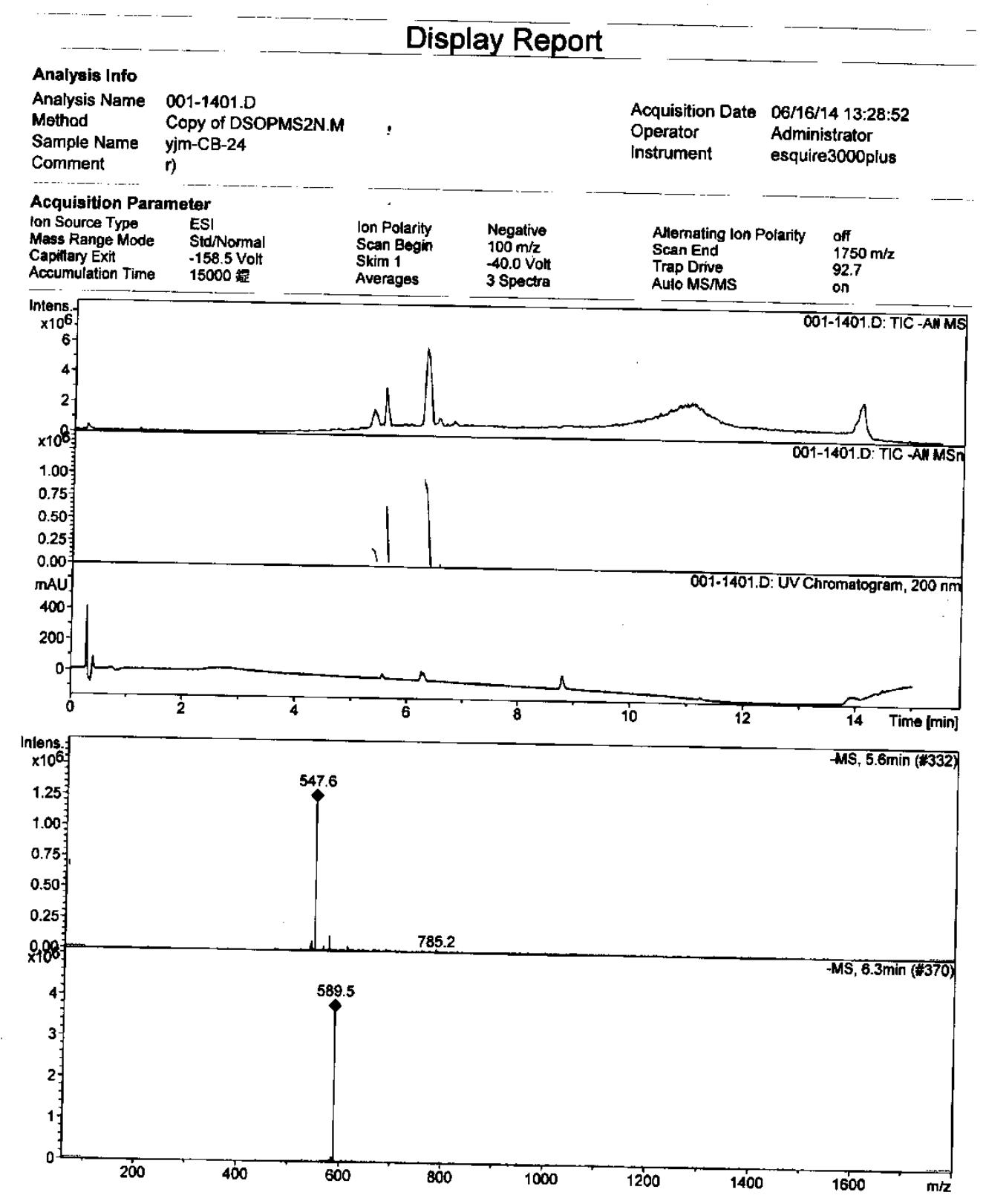


Figure S124. HRESI(+)MS spectrum of cibacciferin G (15)

Elemental Composition Report

Page 1

Single Mass Analysis

Tolerance $=5.0$ PPM DBE: $\min =-1.5, \max =50.0$

Element prediction: Off

Number of isotope peaks used for i-FIT $=3$

Monoisotopic Mass, Even Electron lons

109 formula(e) evaluated with 1 results within limits (üp to 50 closest results for each mass)

Elements Used:

$\begin{array}{lll}\text { C: } 5-80 & \text { H: } 2-120 & \text { O: } 0-20\end{array}$

CB-24

LCT PXE KE324

05-Dec-2013

1: TOF MS ESt

CB-24_1205 9 (0.196) AM2 (Ar, 10000.0.0.00.1.00); ABS; Cm (8:25)

$1.250+004$

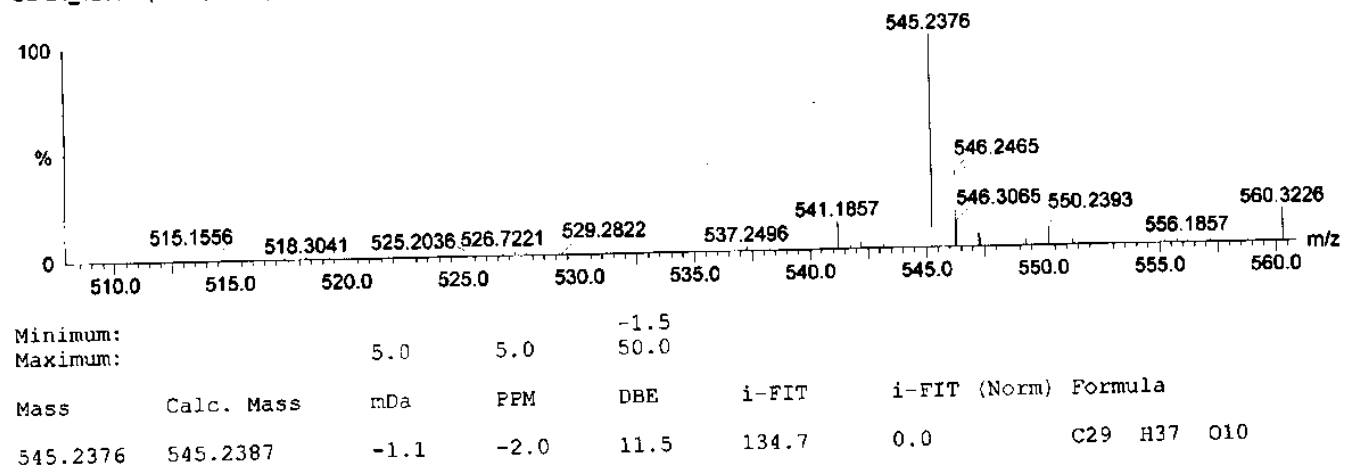


Figure S125. IR spectrum of cibacciferin G (15)

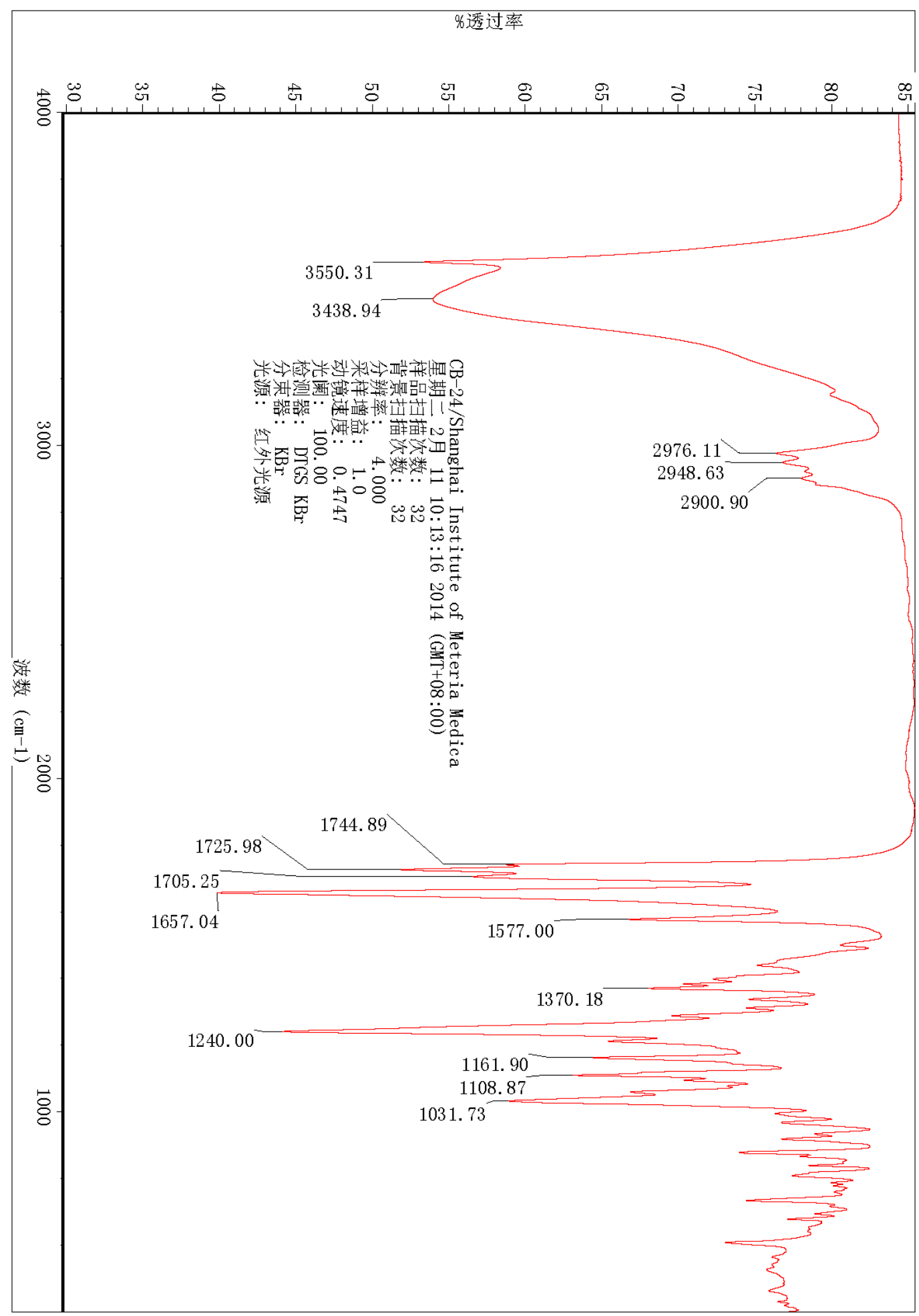


Figure S126. UV spectrum of cibacciferin G (15)

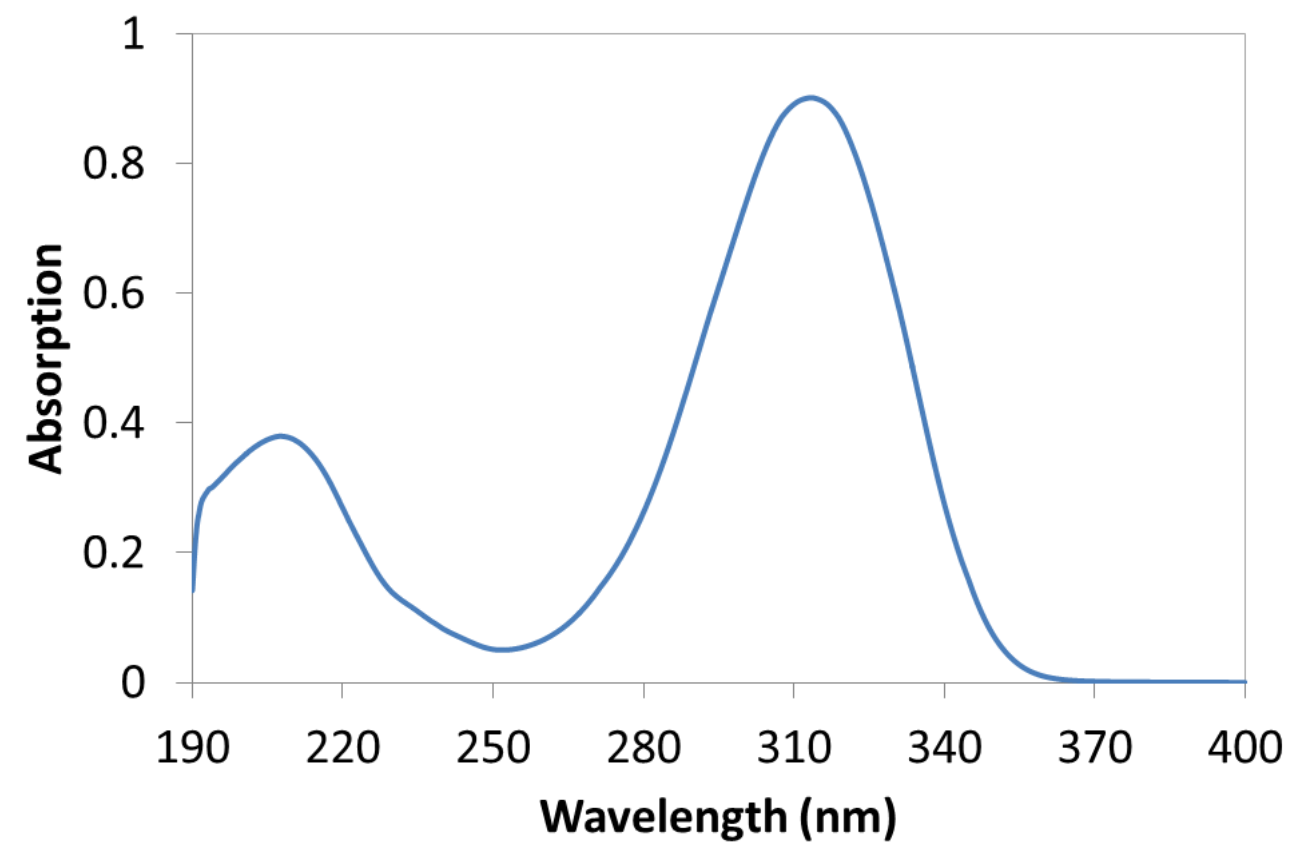


Figure S127. ${ }^{1} \mathrm{H}$ NMR spectrum of cibacciferin $\mathrm{H}(\mathbf{1 6})$ in $\mathrm{CDCl}_{3}$

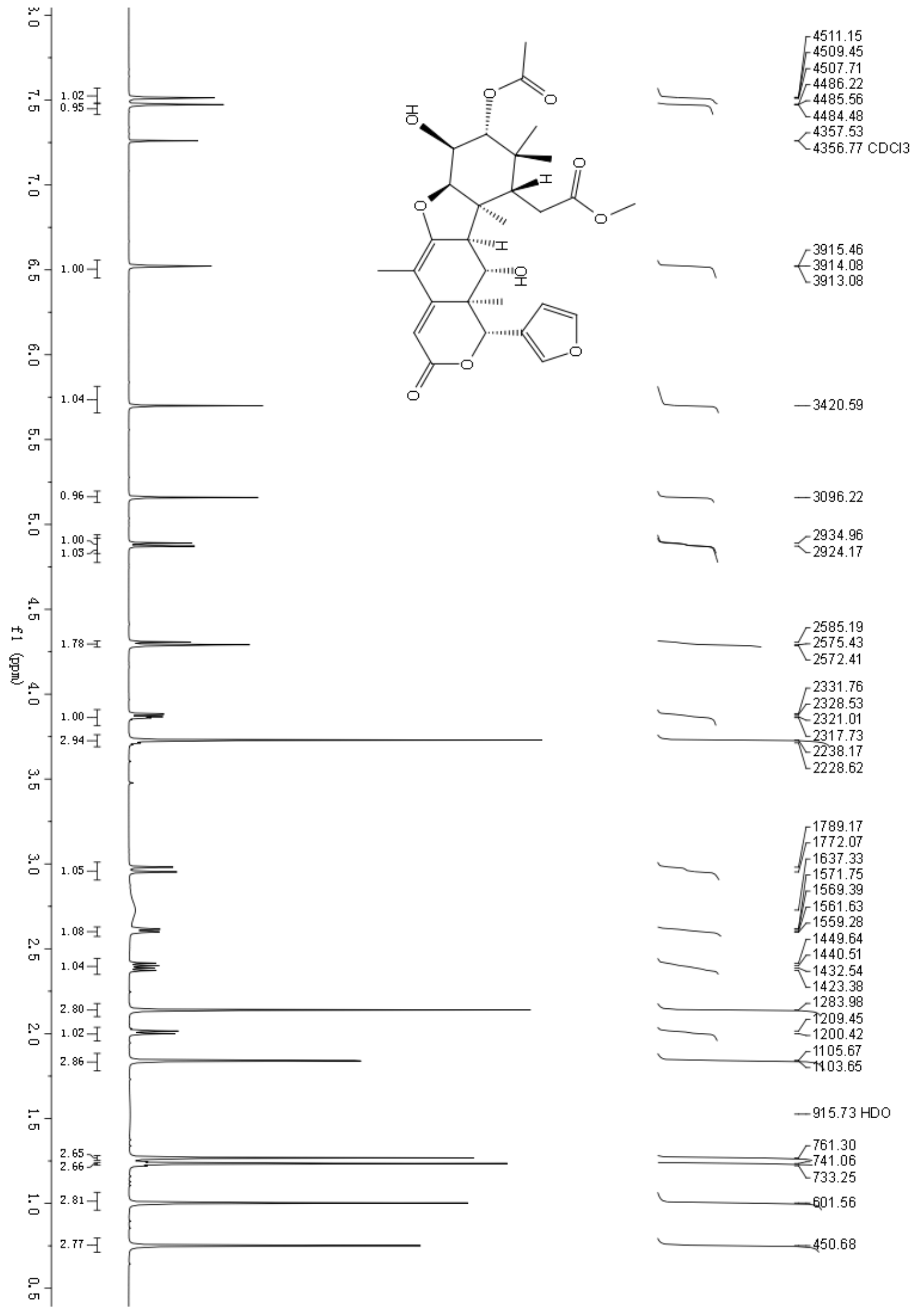


Figure S128. ${ }^{13} \mathrm{C}$ NMR spectrum of cibacciferin $\mathrm{H}(\mathbf{1 6})$ in $\mathrm{CDCl}_{3}$

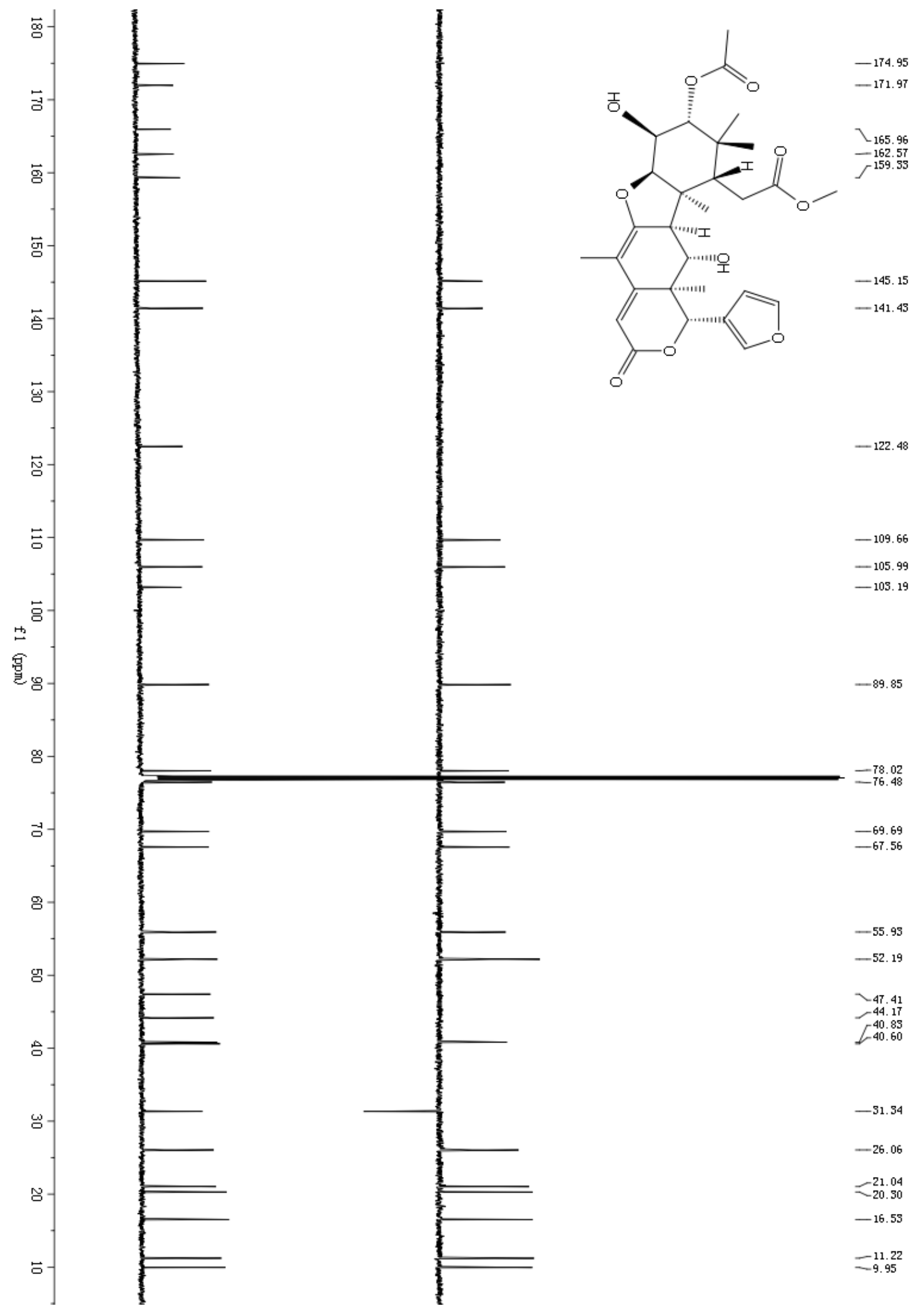


Figure S129. HSQC spectrum of cibacciferin $\mathrm{H}(\mathbf{1 6})$ in $\mathrm{CDCl}_{3}$

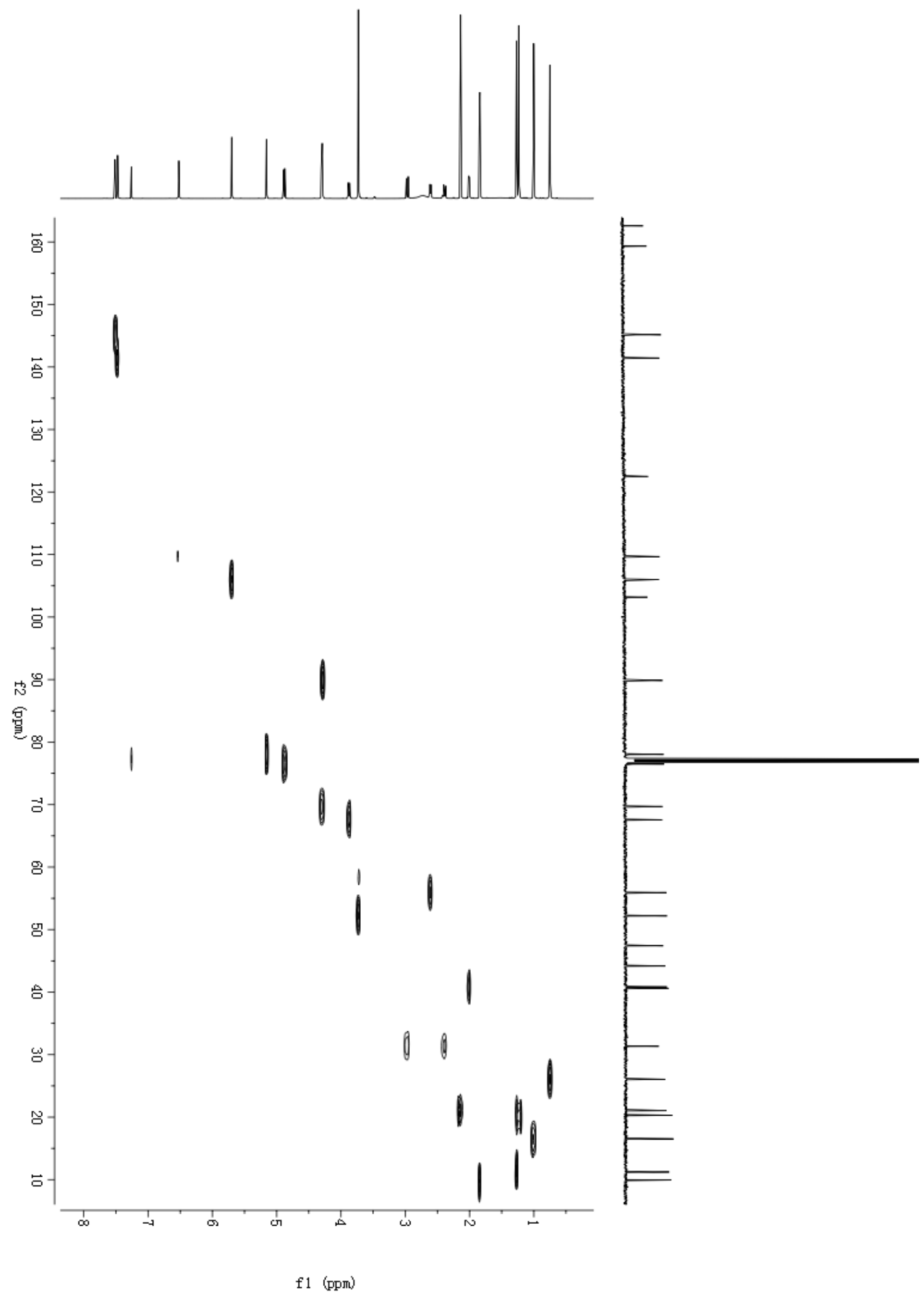


Figure S130. $\mathrm{HMBC}$ spectrum of cibacciferin $\mathrm{H}(\mathbf{1 6})$ in $\mathrm{CDCl}_{3}$

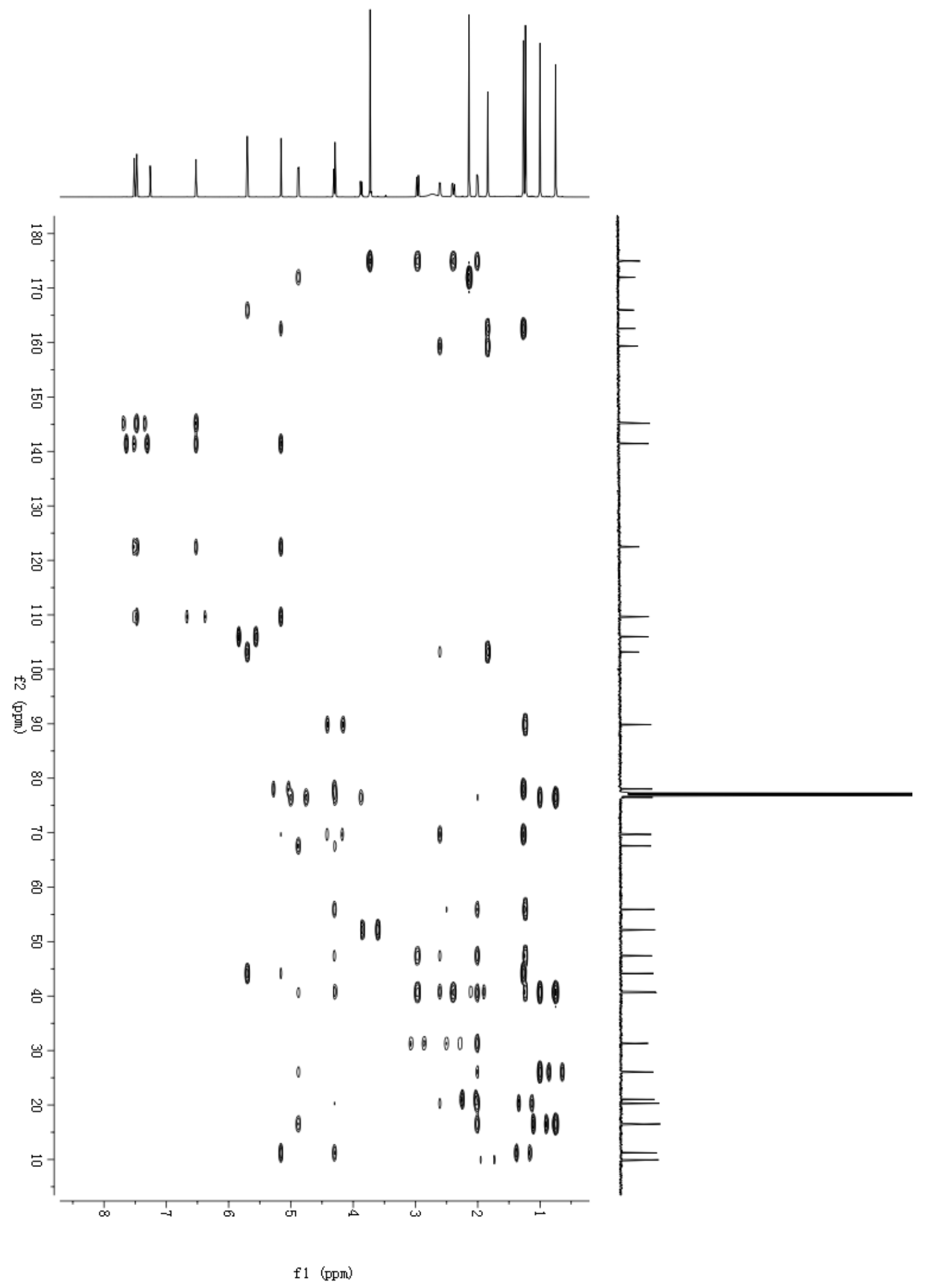


Figure S131. ESI(+)MS spectrum of cibacciferin H (16)

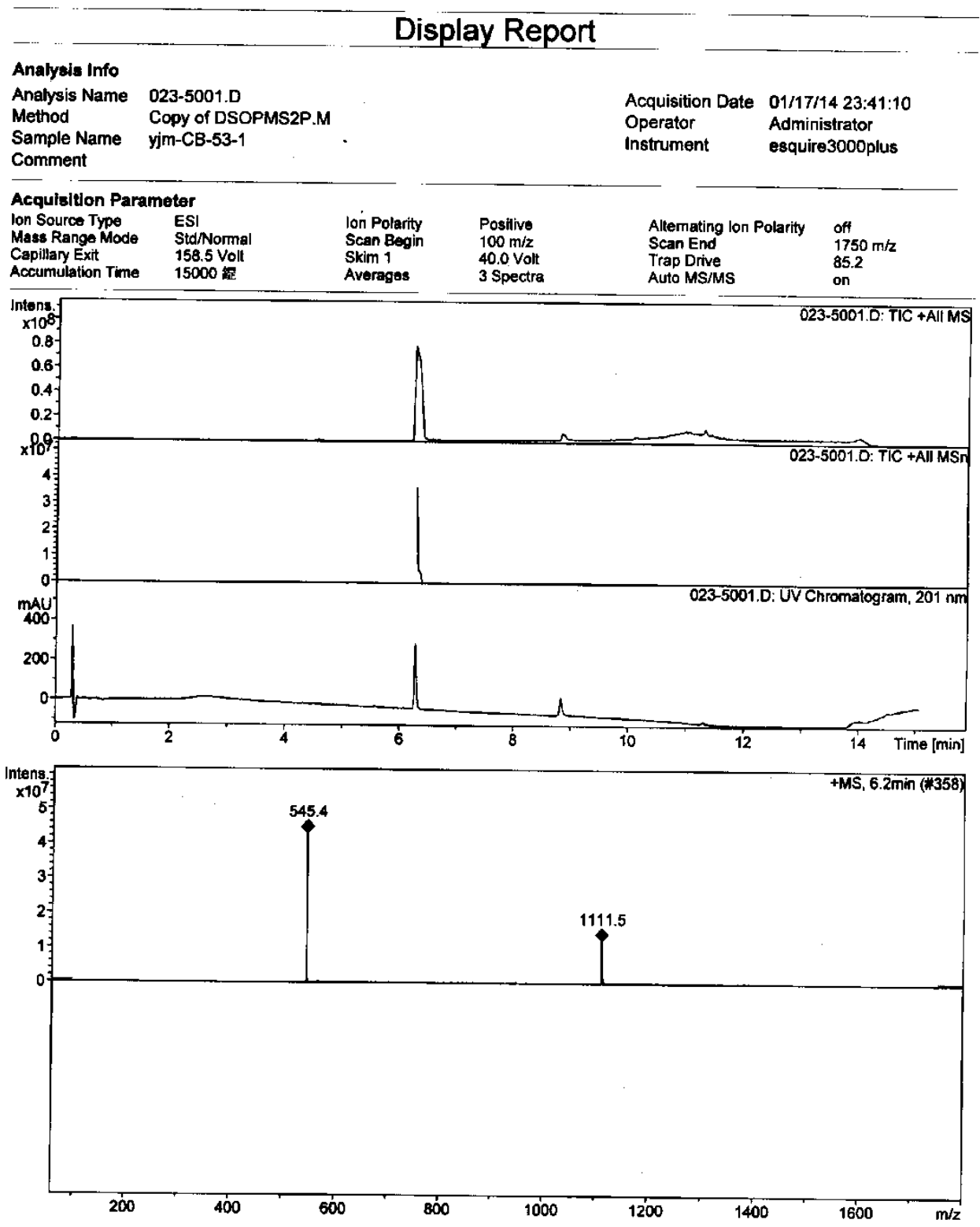

Bruker Daltonics DataAnalysis 3.1 
Figure S132. ESI(-)MS spectrum of cibacciferin H (16)

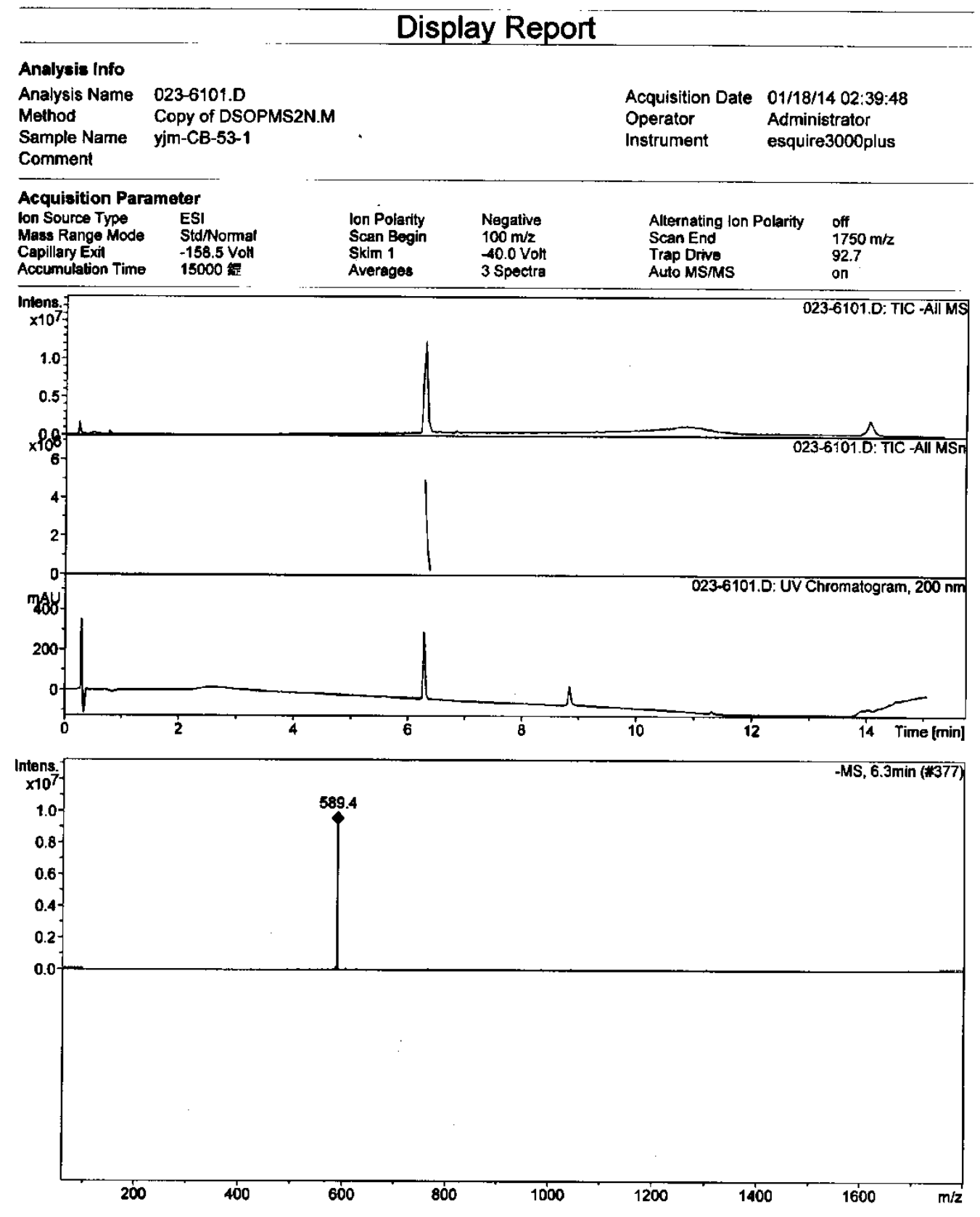


Figure S133. HRESI(+)MS spectrum of cibacciferin H (16)

Elemental Composition Report

Page 1

Single Mass Analysis

Tolerance $=5.0$ PPM / DBE: $\min =-1.5, \max =50.0$

Element prediction: Off

Number of isotope peaks used for $\mathrm{i}-\mathrm{FIT}=3$

Monoisotopic Mass, Even Electron lons

216 formula(e) evaluated with 1 results within limits (up to 50 closest results for each mass)

Elements Used:

$\begin{array}{llll}\text { C: } 5-80 & \text { H: } 2-120 & \text { O: } 0-20 & \mathrm{Na}: 0-1\end{array}$

CB-53-1 . LCT PXE KE324

CB-53-1_0505 S (0.195) AM2 (Ar, 10000.0,0.00,1.00); ABS; Cm \{5:17)

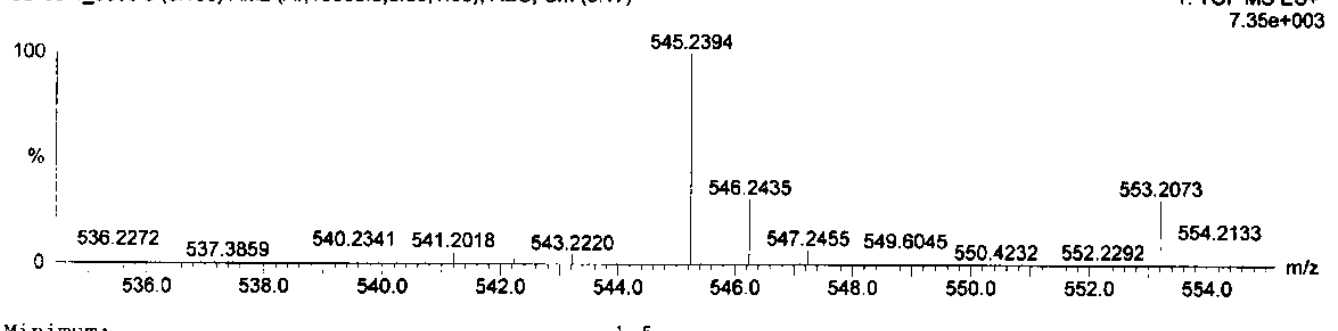

Minimum:

Maximium: $\quad 5.0 \quad 5.0 \quad \frac{-1.5}{50}$

Máss Calc. Mass mDa PPM DBE i-EIT i-FIT (Norm) Formula

$\begin{array}{llllllllll}545.2394 & 545.2387 & 0.7 & 1.3 & 11.5 & 108.8 & 0.0 & \text { C29 } & \text { H37 } & 010\end{array}$ 
Figure S134. IR spectrum of cibacciferin H (16)

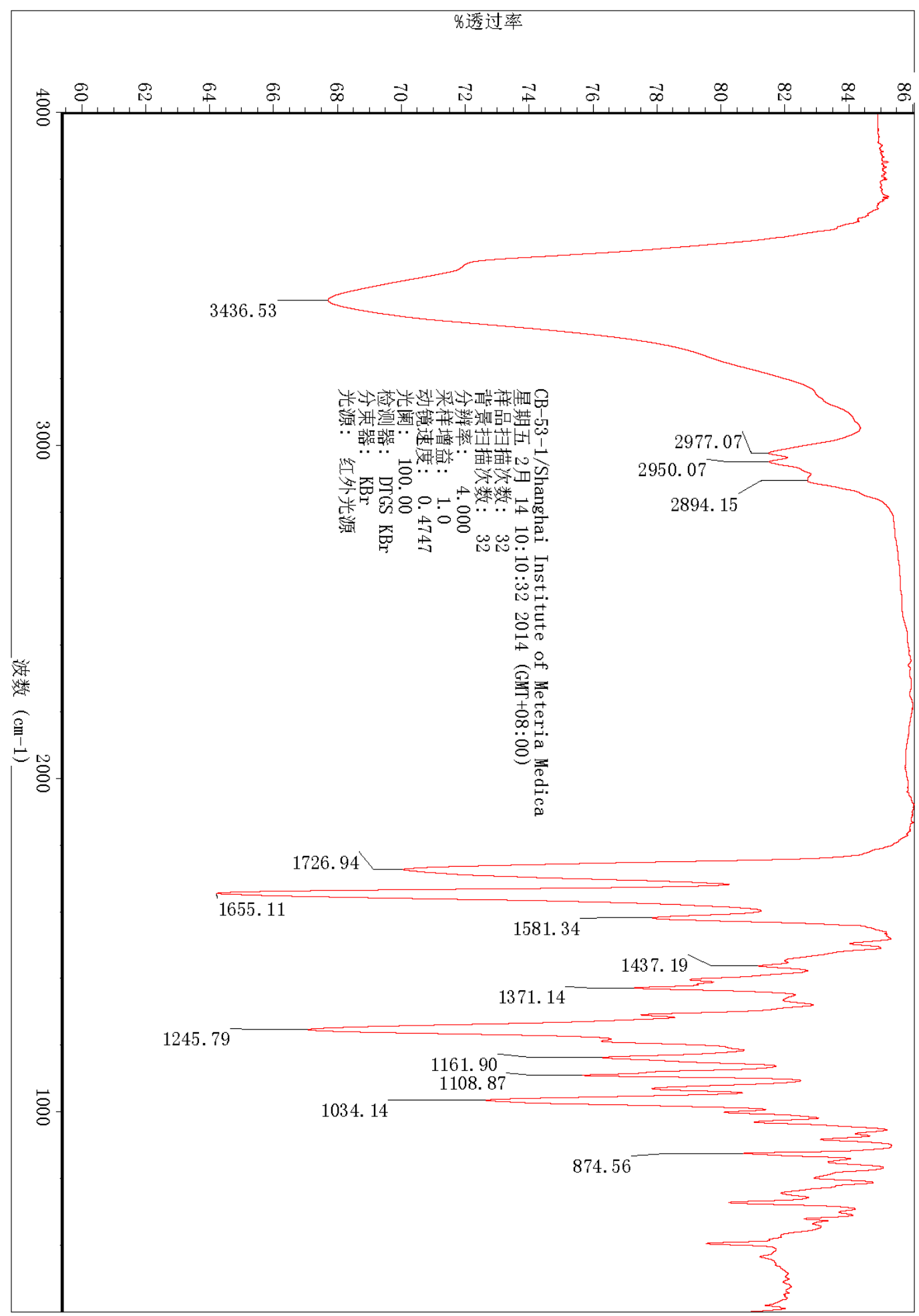


Figure S135. UV spectrum of cibacciferin H (16)

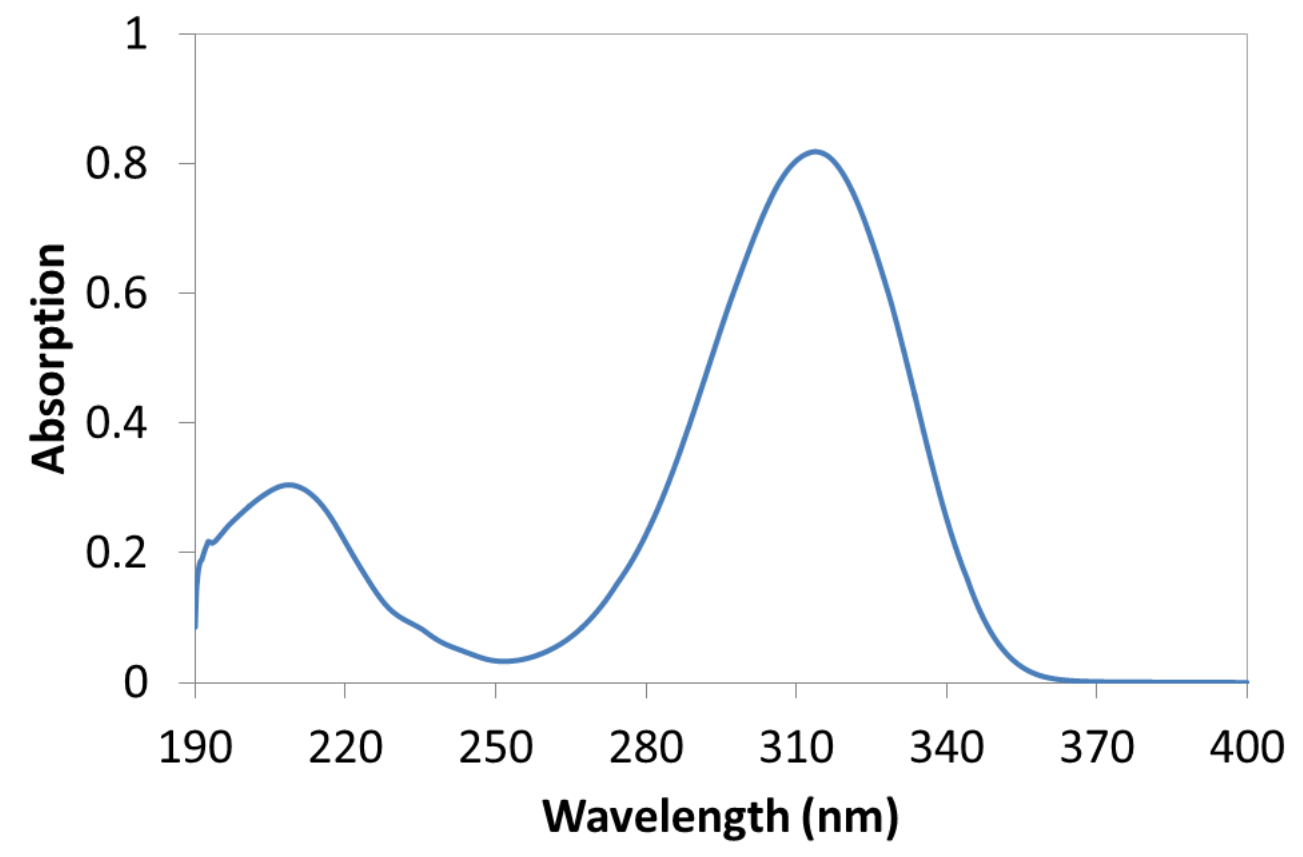


Figure S136. ${ }^{1} \mathrm{H}$ NMR spectrum of 12-dehydroxycibacciferin $\mathrm{H}(17)$ in $\mathrm{CDCl}_{3}$

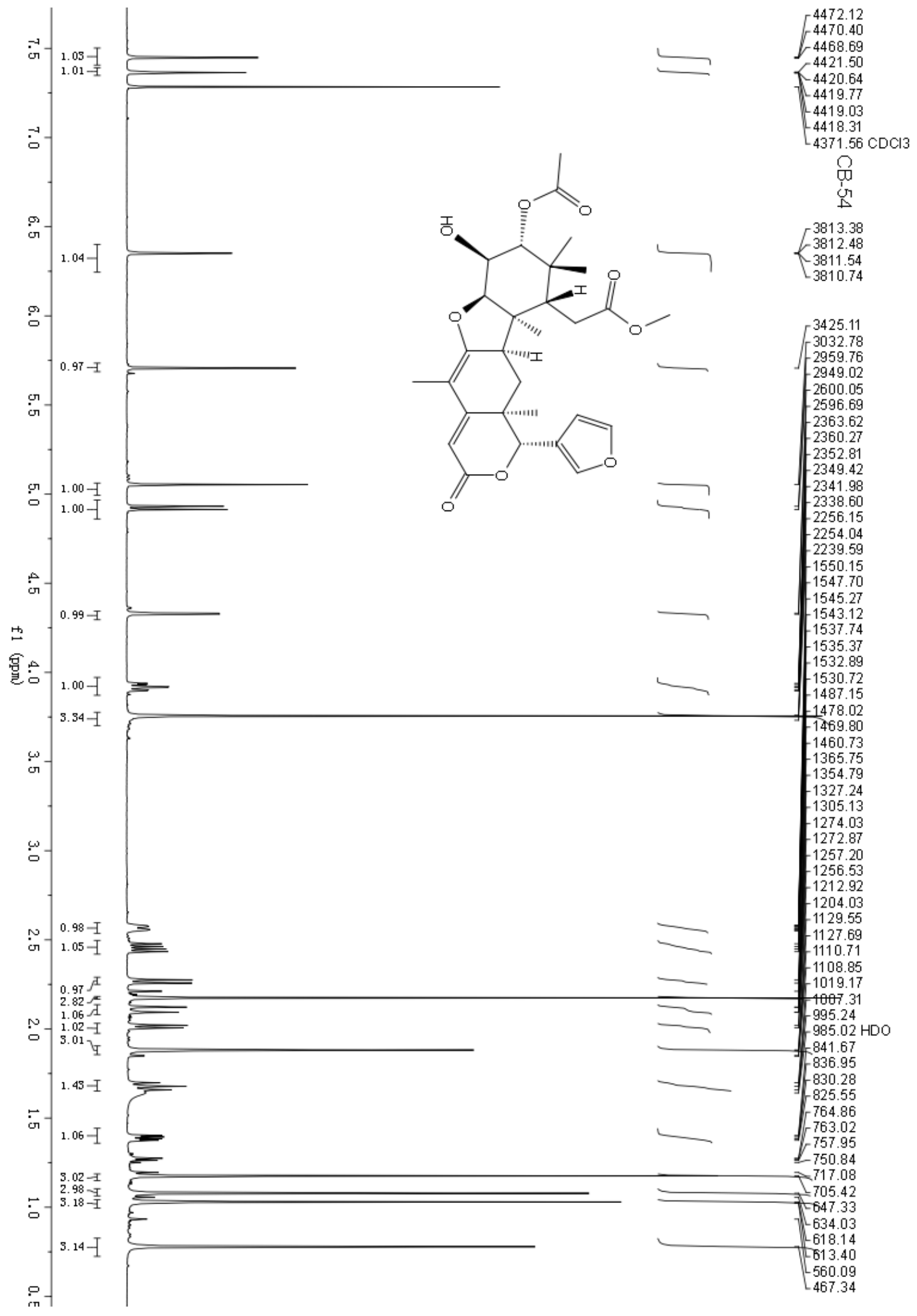


Figure S137. ${ }^{13} \mathrm{C}$ NMR spectrum of 12-dehydroxycibacciferin $\left.\mathrm{H}(\mathbf{1 7})\right)$ in $\mathrm{CDCl}_{3}$

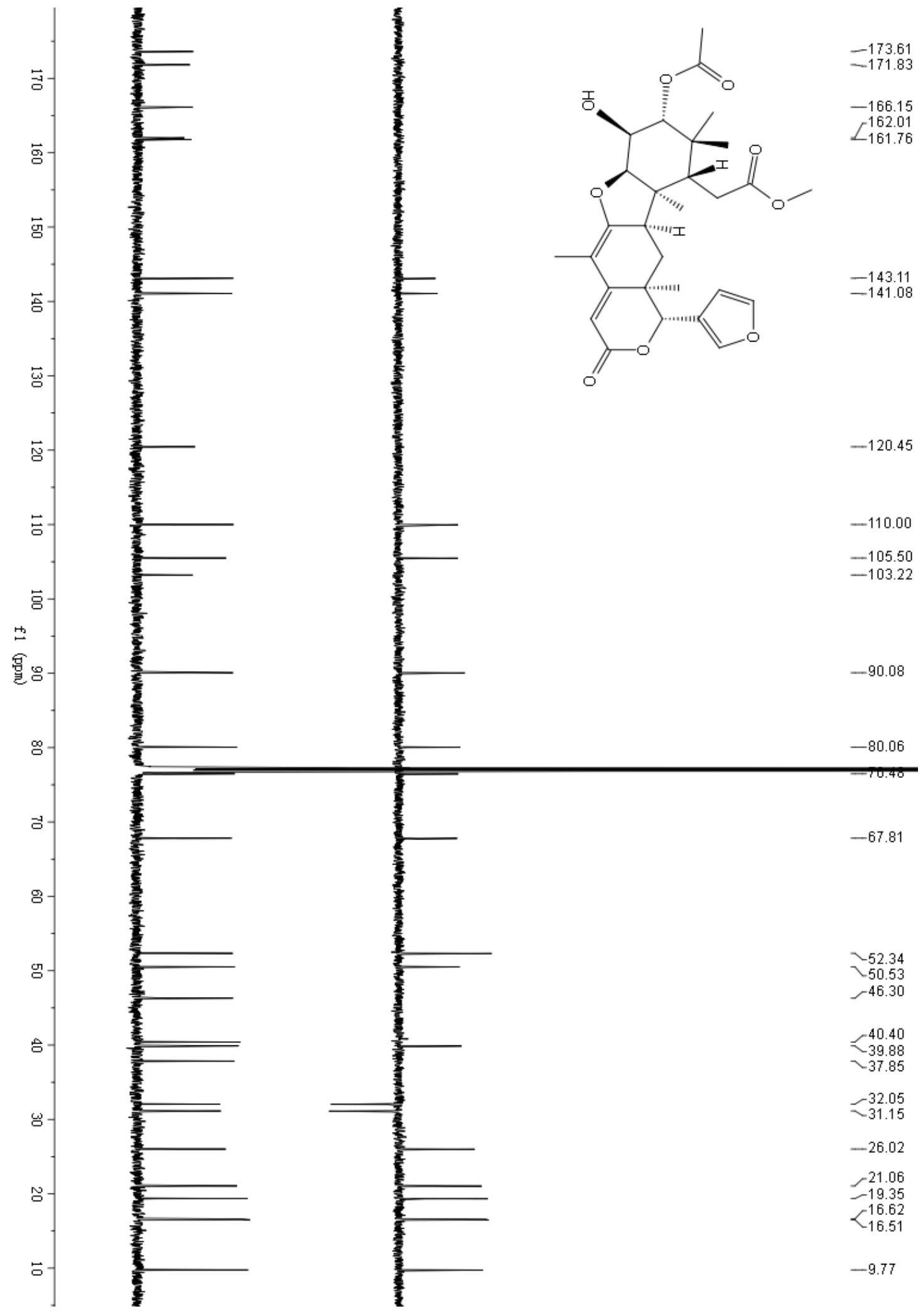


Figure S138. HSQC spectrum of 12-dehydroxycibacciferin $\mathrm{H}(17)$ in $\mathrm{CDCl}_{3}$

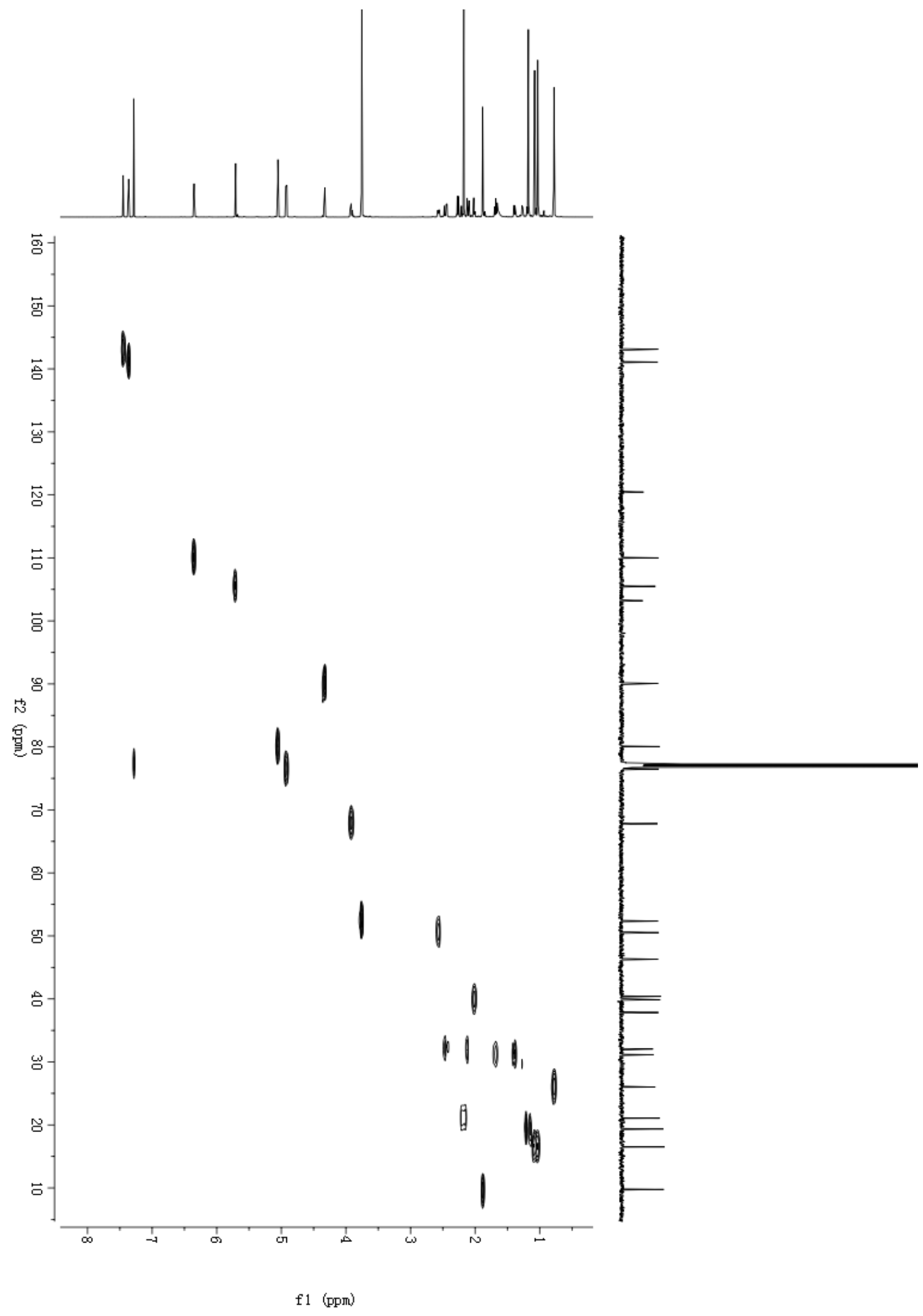


Figure S139. HMBC spectrum of 12-dehydroxycibacciferin $\mathrm{H}(17)$ in $\mathrm{CDCl}_{3}$

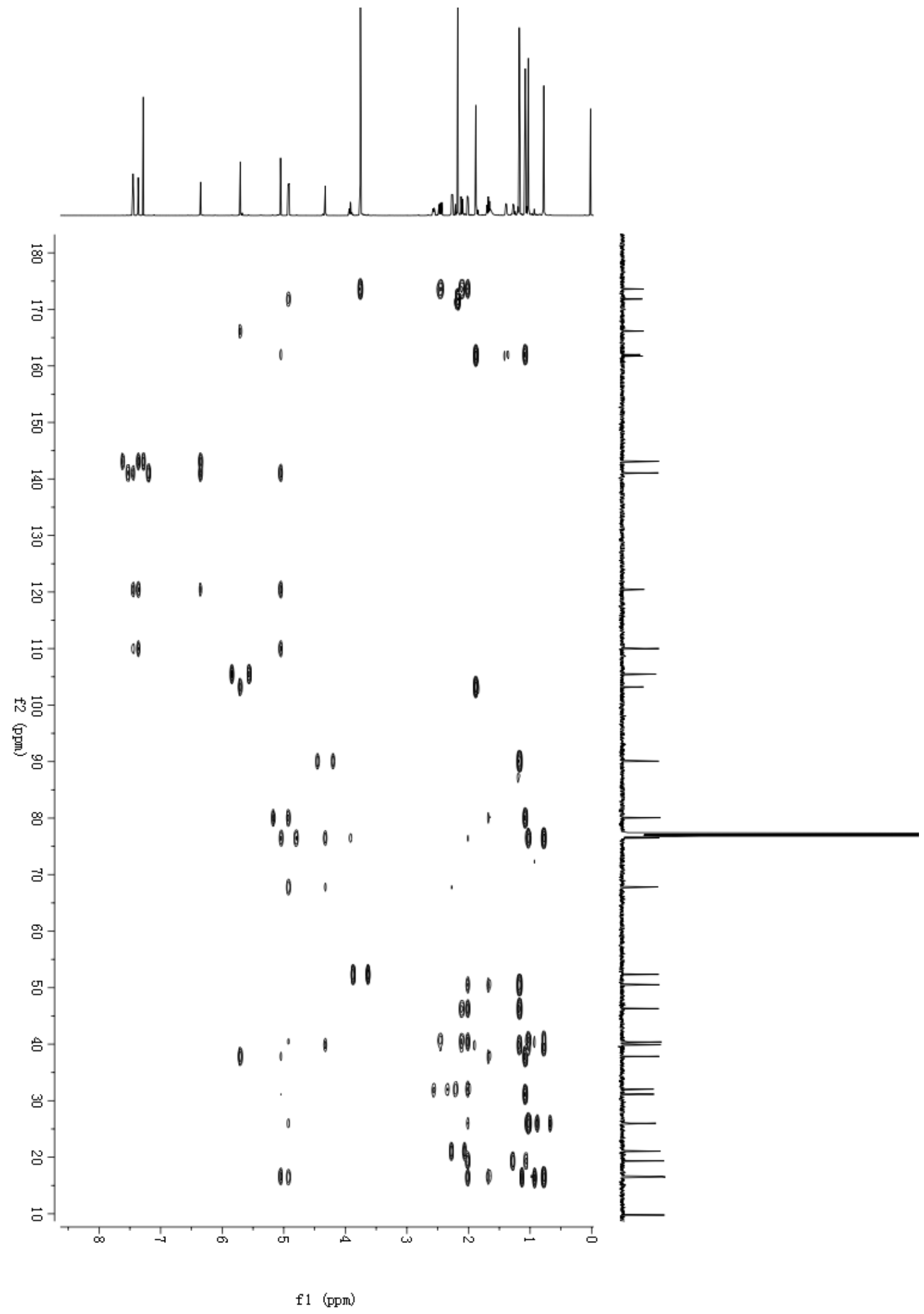


Figure S140. ESI(+)MS spectrum of 12-dehydroxycibacciferin H (17)

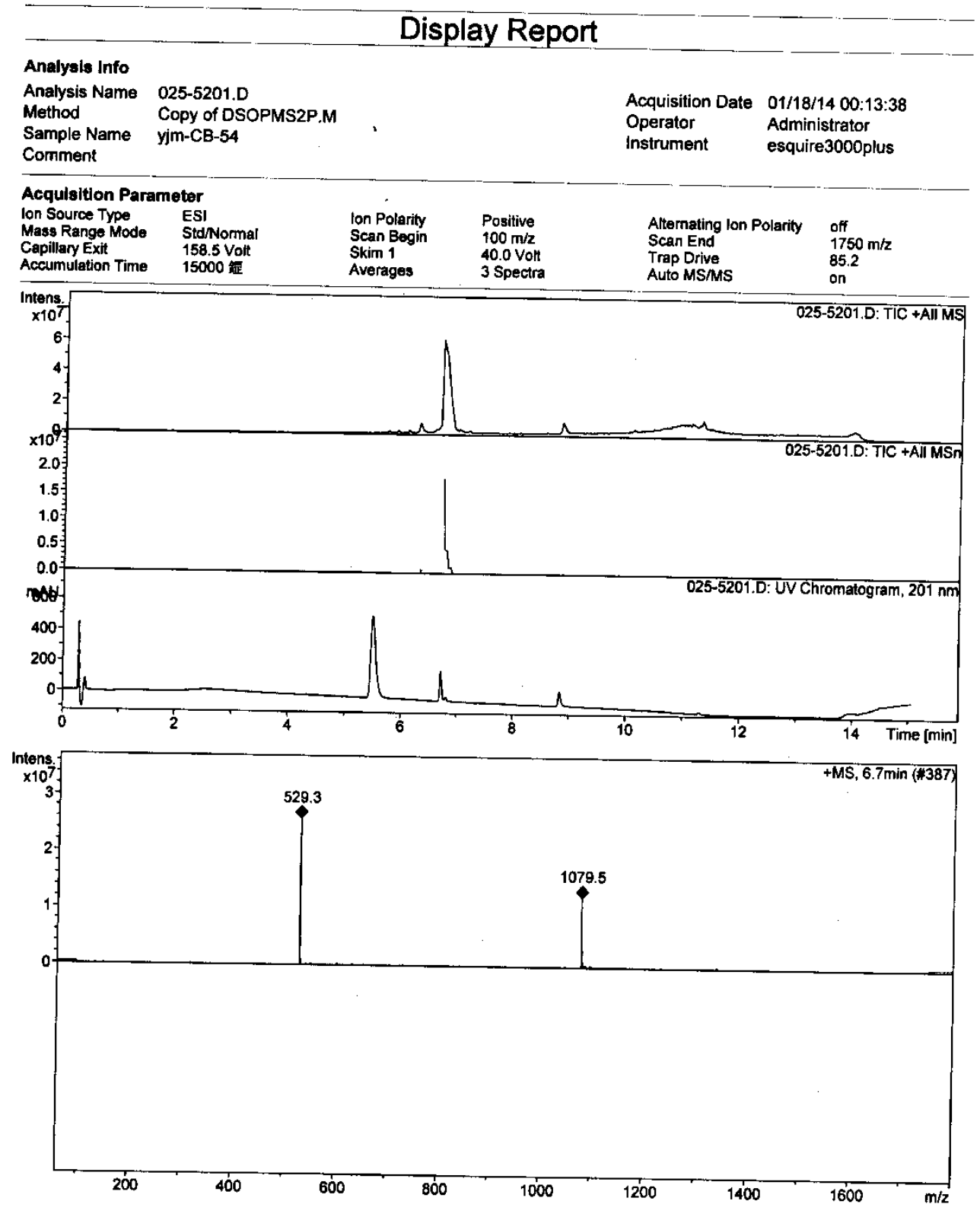


Figure S141. ESI(-)MS spectrum of 12-dehydroxycibacciferin H (17)

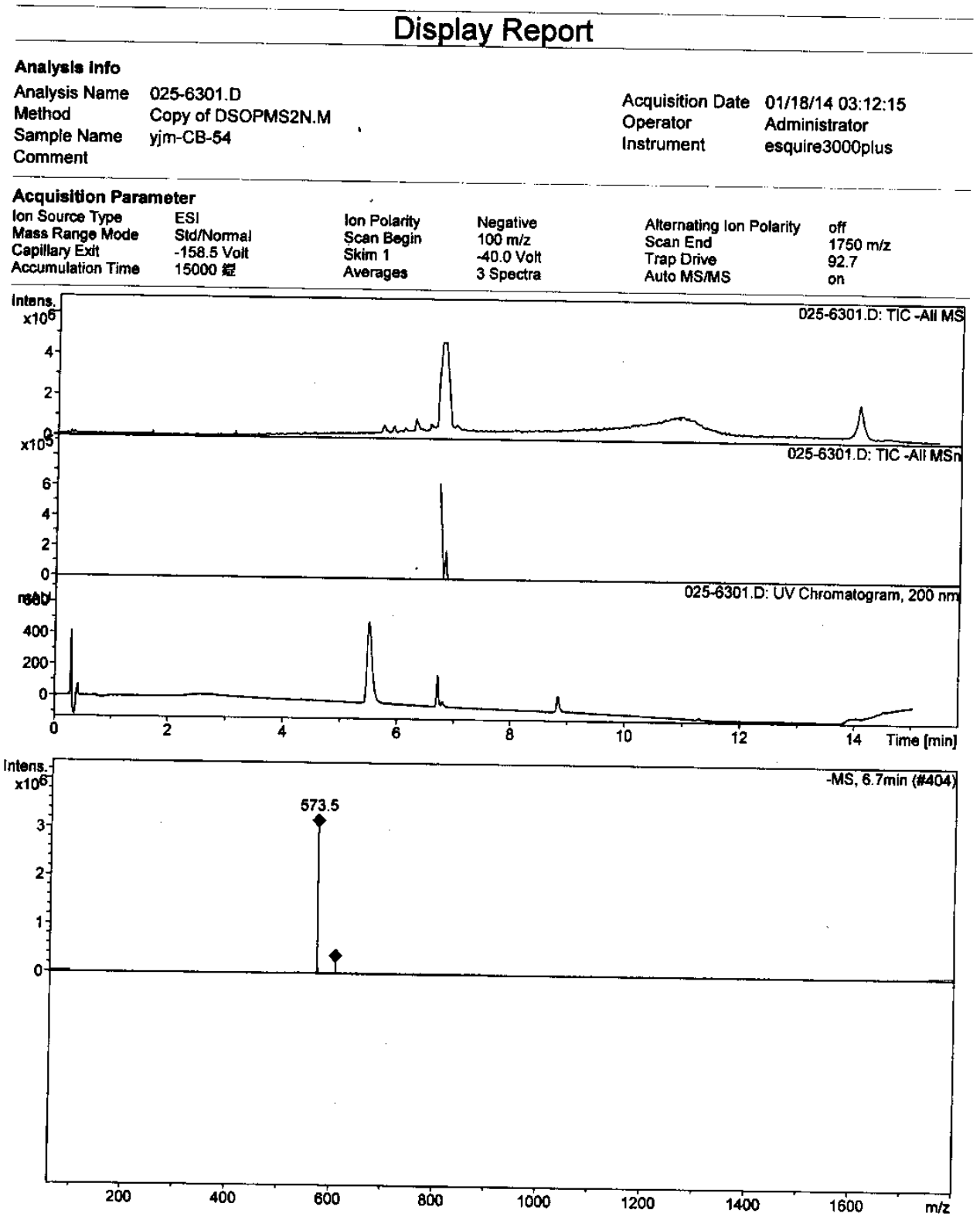


Figure S142. HRESI(+)MS spectrum of 12-dehydroxycibacciferin H (17)

\section{Elemental Composition Report}

Page 1

\section{Single Mass Analysis}

Tolerance $=3.0 \mathrm{PPM} / \mathrm{DBE}: \min =-1.5, \max =50.0$

Element prediction: Off

Number of isotope peaks used for i-FIT $=3$

Monoisotopic Mass, Even Electron lons

208 formula(e) evaluated with 1 results within limits (up to 50 closest results for each mass)

Elements Used:

$\begin{array}{llll}\text { C: } 5-80 & \text { H: } 2-120 & \text { O: } 0-20 & \text { Na: } 0-1\end{array}$

CB-54 54 LCT PXE KE324
L

Q6-May-2014

1: TOF MS ES+

CB-54_0506 13 (0.283) AM2 (Ar, 10000.0,0.00,1.00); ABS; Cm (5:22)

$1.54 \mathrm{e}+004$

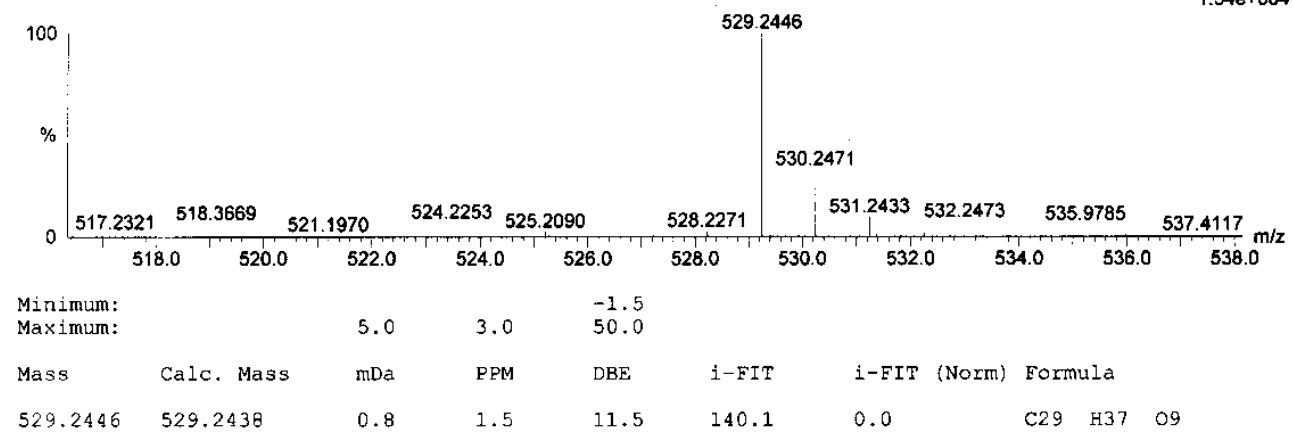


Figure S143. IR spectrum of 12-dehydroxycibacciferin H (17)

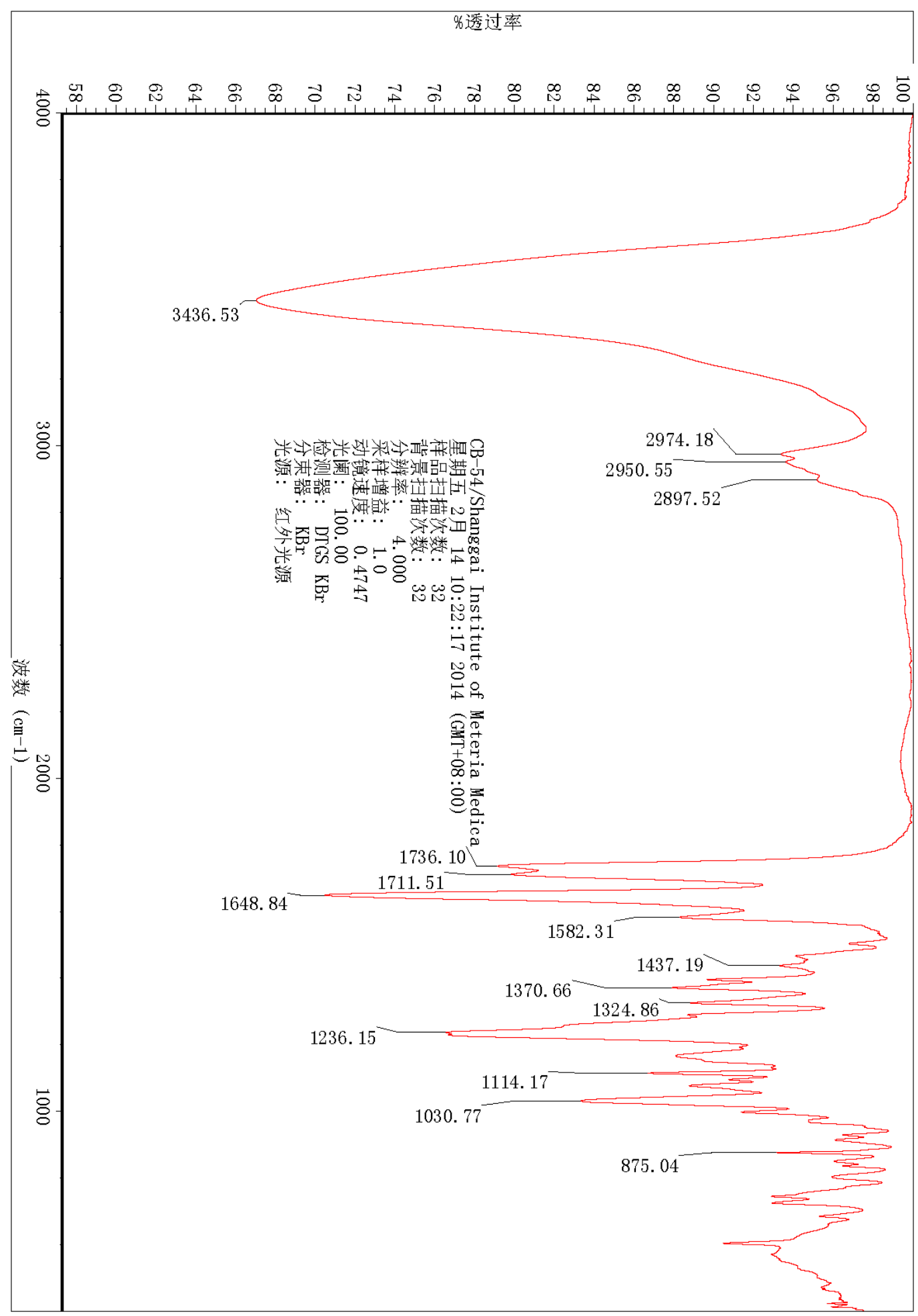


Figure S144. UV spectrum of 12-dehydroxycibacciferin H (17)

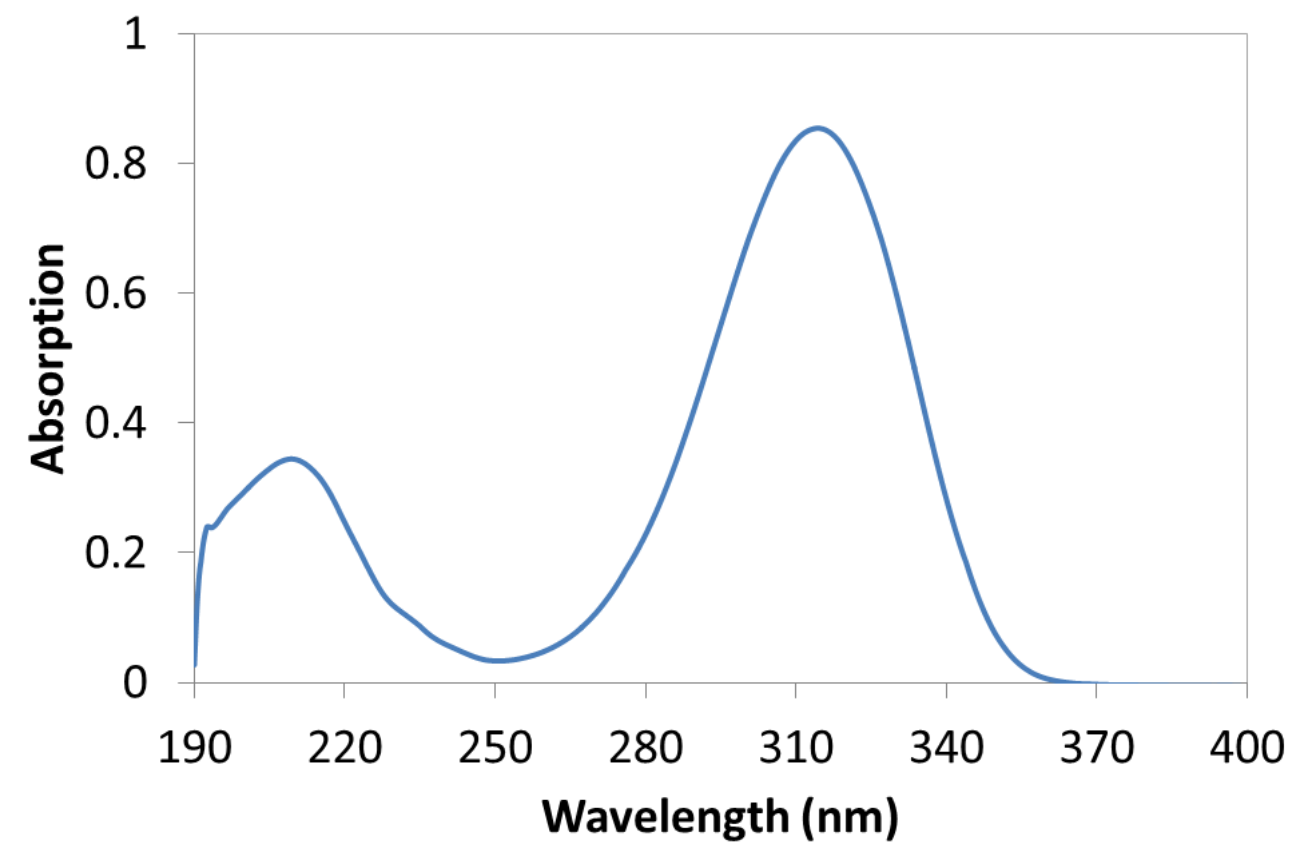


Figure S145. ${ }^{1} \mathrm{H}$ NMR spectrum of cibacciferin I (18) in $\mathrm{CDCl}_{3}$
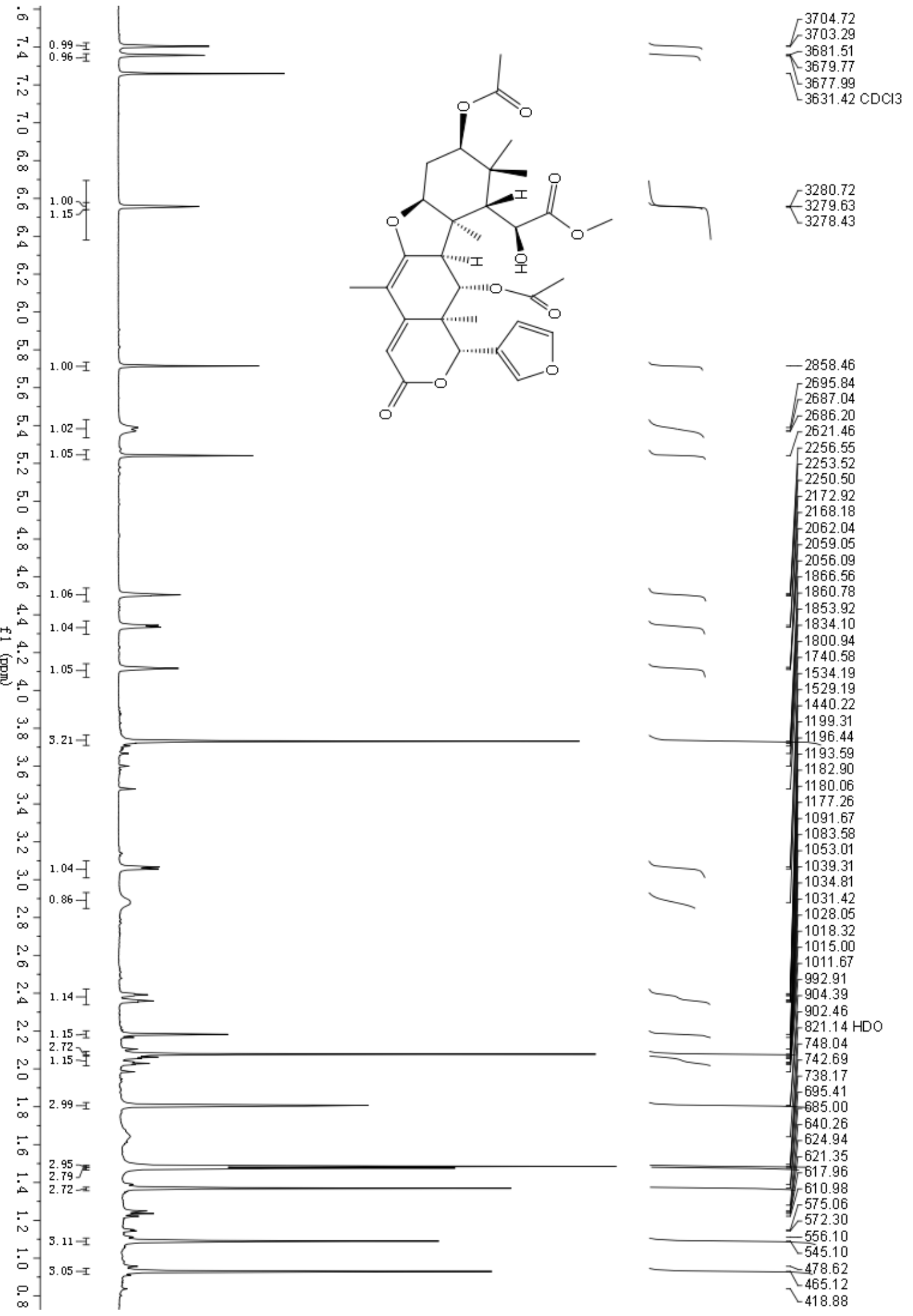
Figure S146. ${ }^{13} \mathrm{C}$ NMR spectrum of cibacciferin I (18) in $\mathrm{CDCl}_{3}$

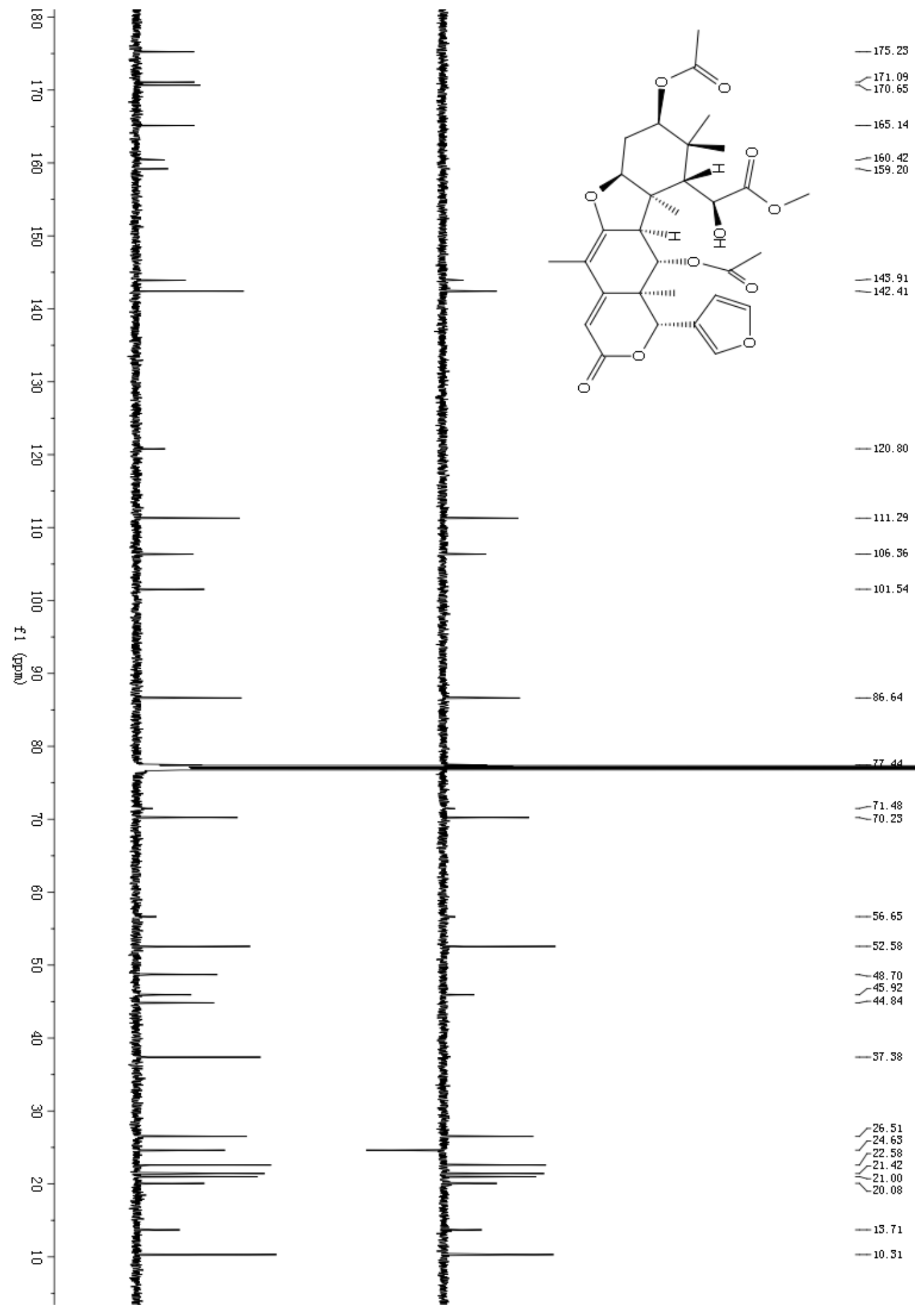


Figure S147. HSQC spectrum of cibacciferin I (18) in $\mathrm{CDCl}_{3}$

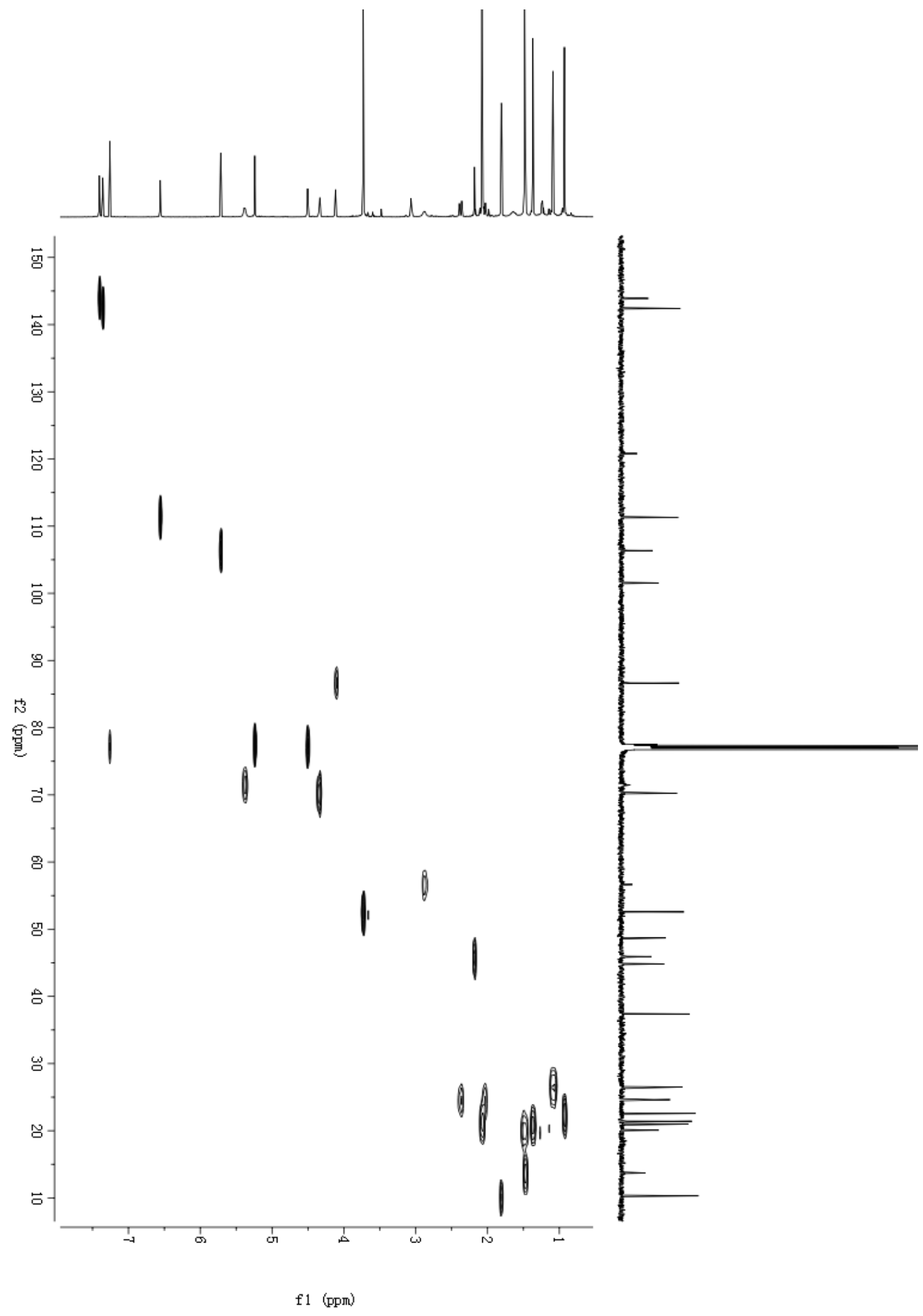


Figure S148. $\mathrm{HMBC}$ spectrum of cibacciferin I (18) in $\mathrm{CDCl}_{3}$

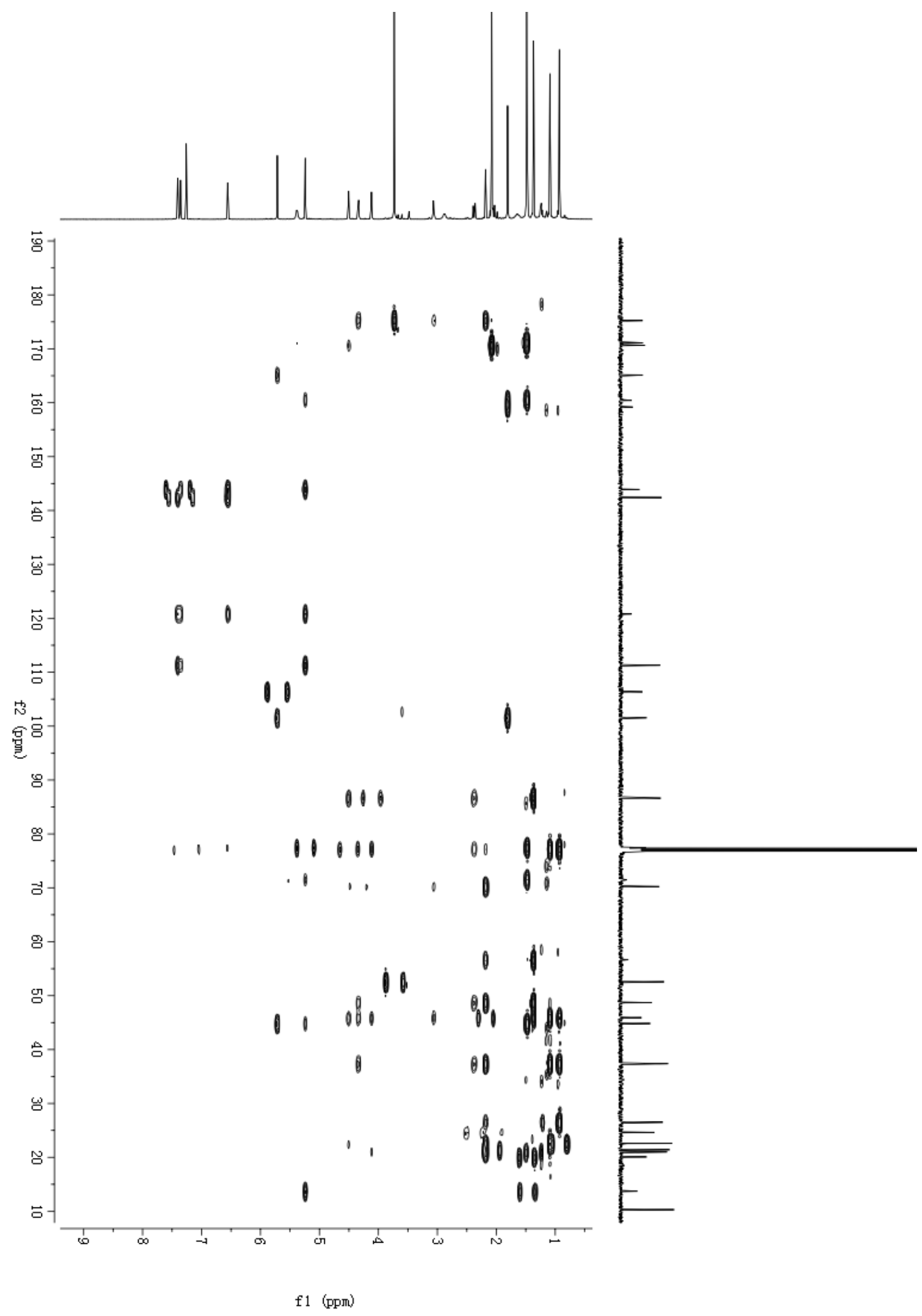


Figure S149. ROESY spectrum of cibacciferin I (18) in $\mathrm{CDCl}_{3}$

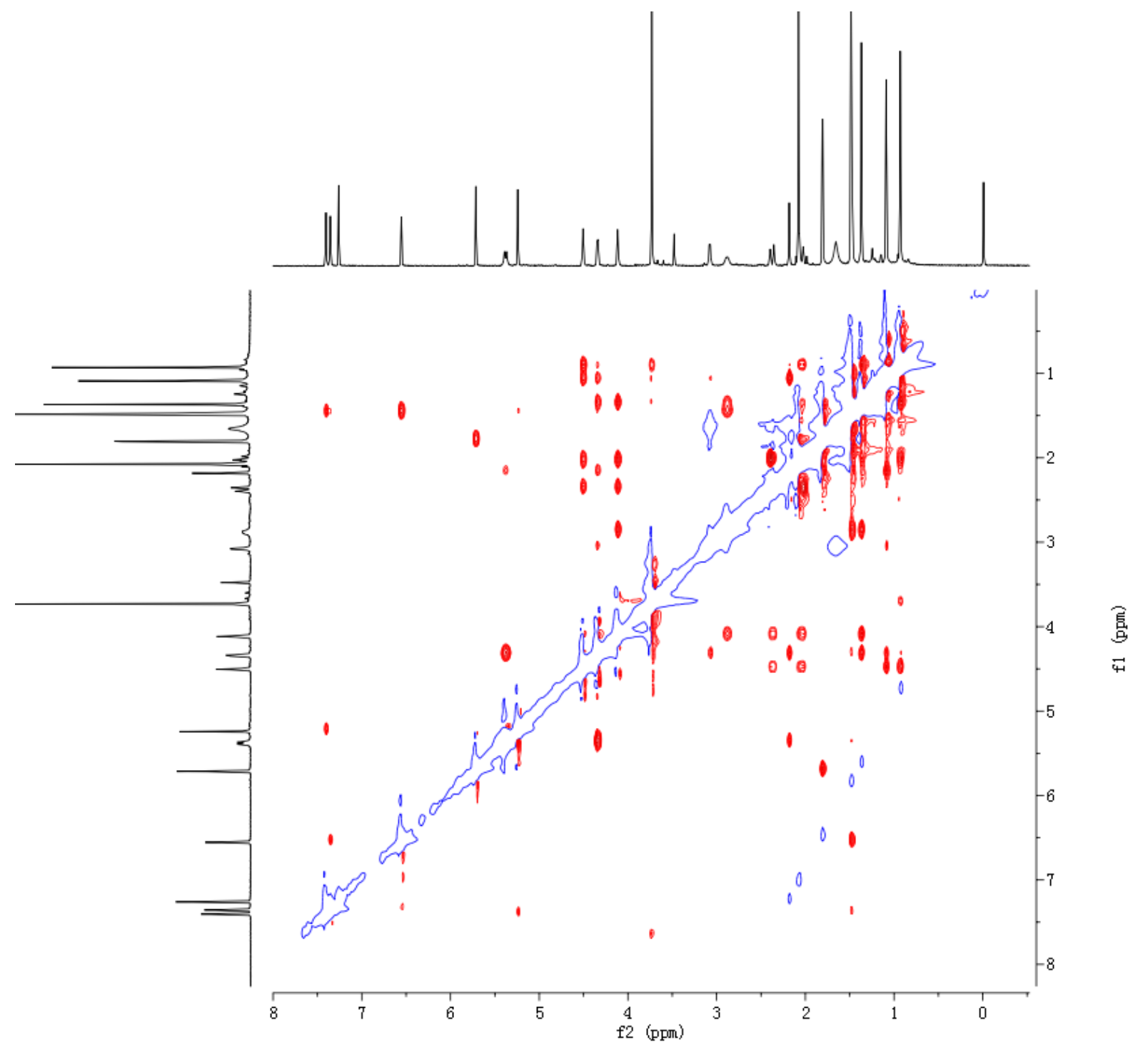


Figure S150. ESI(+)MS spectrum of cibacciferin I (18)

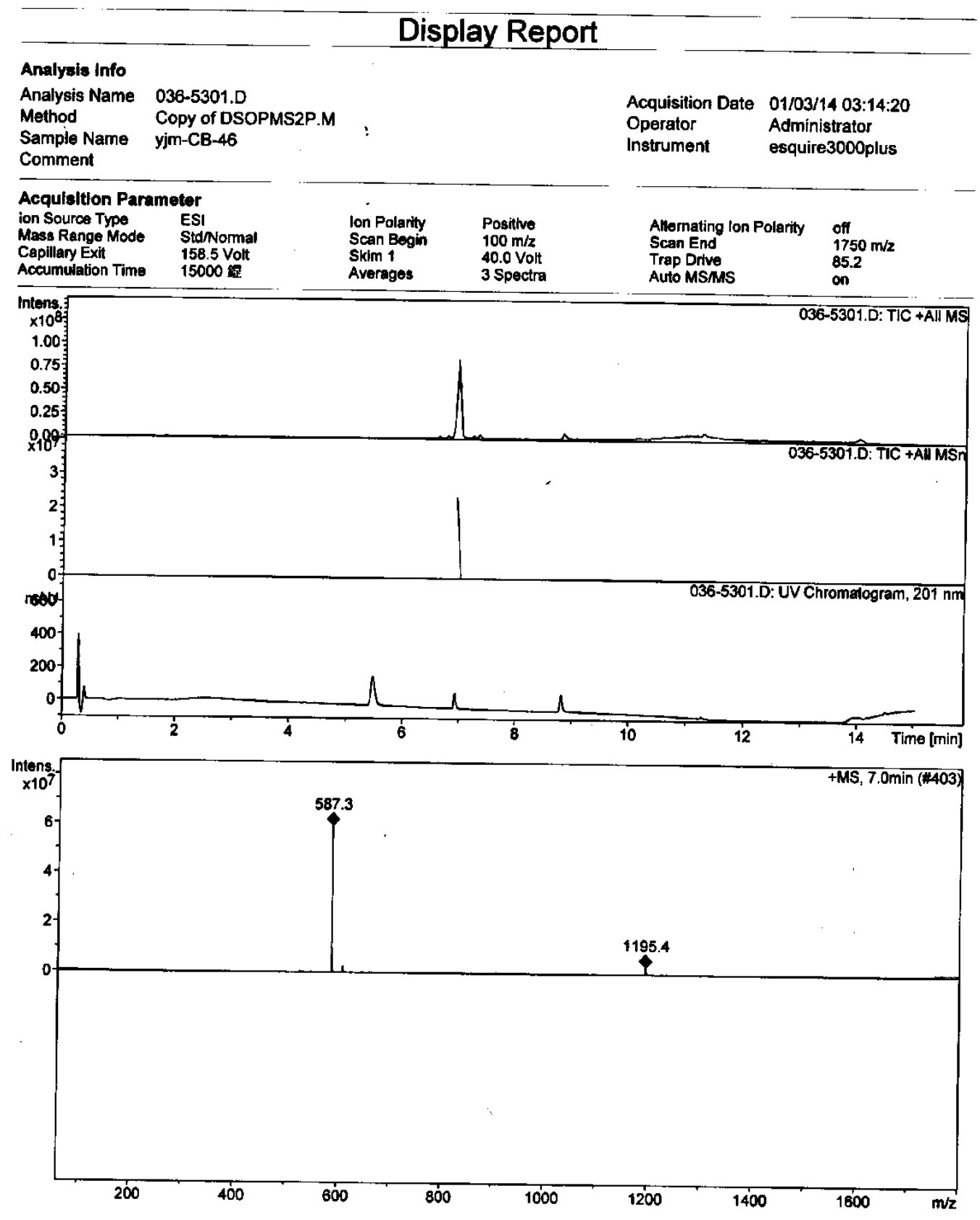


Figure S151. ESI(-)MS spectrum of cibacciferin I (18)

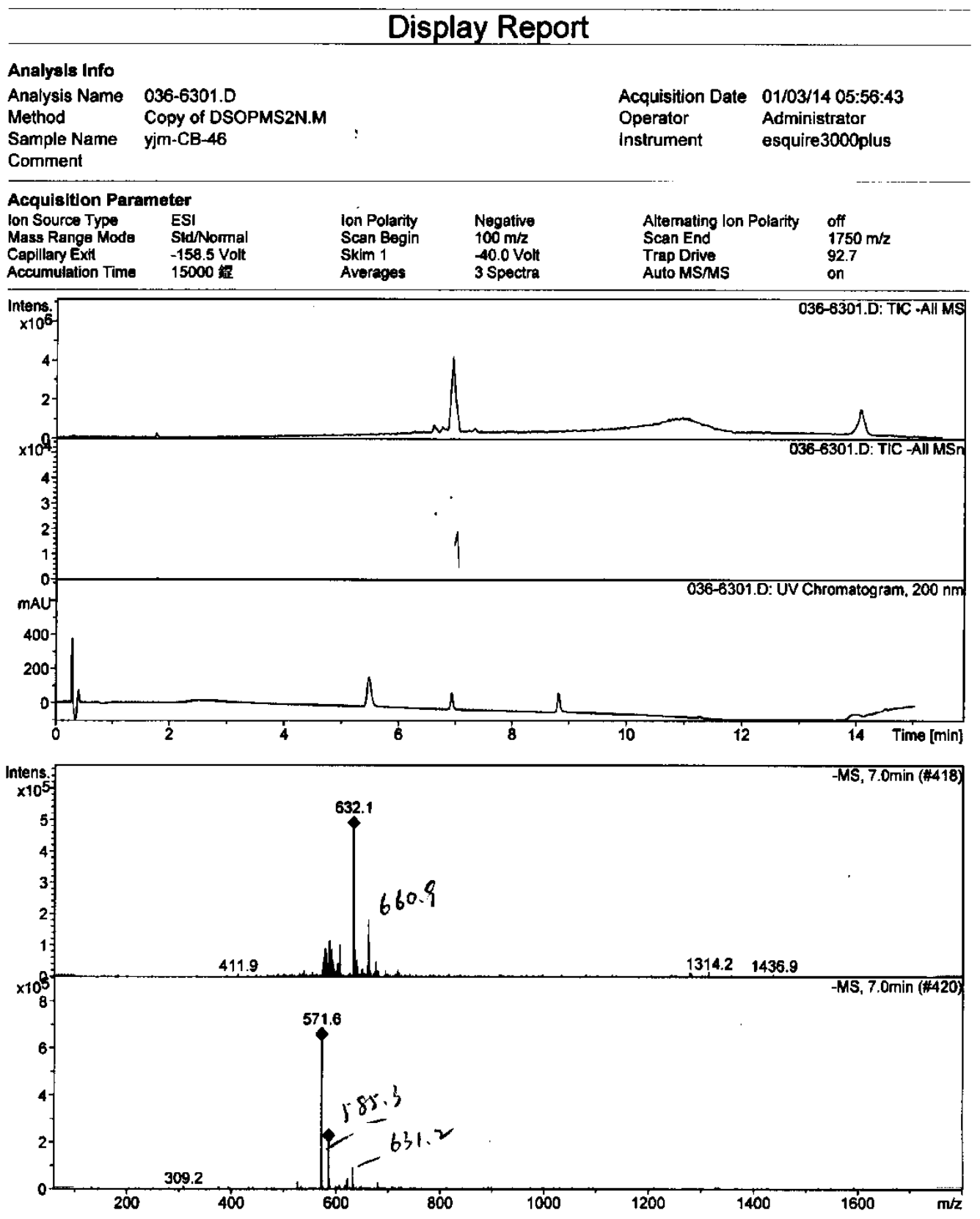


Figure S152. HRESI(+)MS spectrum of cibacciferin I (18)

Elemental Composition Report

Page 1

Single Mass Analysis

Tolerance $=3.0 \mathrm{PPM} / \mathrm{DBE}: \min =-1.5, \max =50.0$

Element prediction: Off

Number of isotope peaks used for i-FIT $=3$

Monoisotopic Mass, Even Electron lons

237 formula(e) evaluated with 1 results within limits '(up to 50 closest results for each mass)

$\begin{array}{llll}\text { C: } 5-80 & \text { H: } 2-120 & \text { O: } 0-20 & \mathrm{Na}: 0-1\end{array}$

\begin{tabular}{l} 
CB-46 LCT PXE KE324 \\
\hline
\end{tabular}

03-Apr-2014

13:46:57

CB-46_20140403 10 (0.196) AM2 (Ar, 10000.0,0.00,1.00); ABS; Cm (6:21)

1: TOF MS ES+

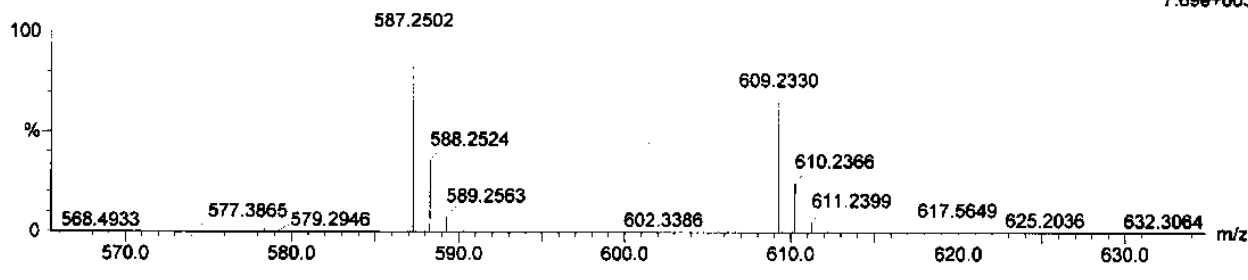

Minimum: -3.5

Maximum: $\quad 5.0 \quad 3.0 \quad 50.0$

Mass Calc. Mass mDa PPM DBE i-EIT i-FIT (Norm) Formula

$\begin{array}{llllllllll}587.2502 & 587.2492 & 1.0 & 1.7 & 12.5 & 81.0 & 0.0 & \text { C31 } & \text { H39 } & 011\end{array}$ 
Figure S153. IR spectrum of cibacciferin I (18)

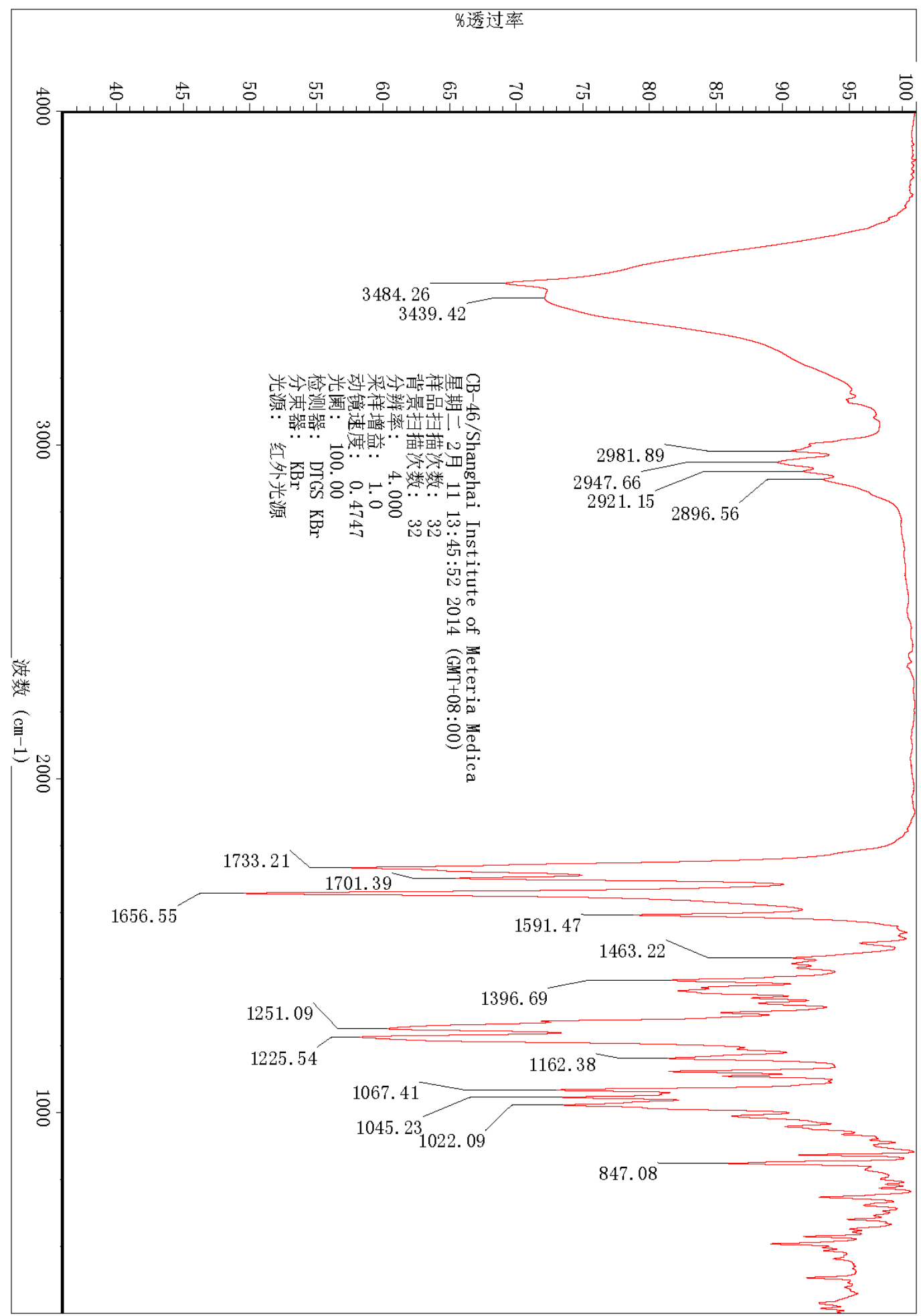


Figure S154. UV spectrum of cibacciferin I (18)

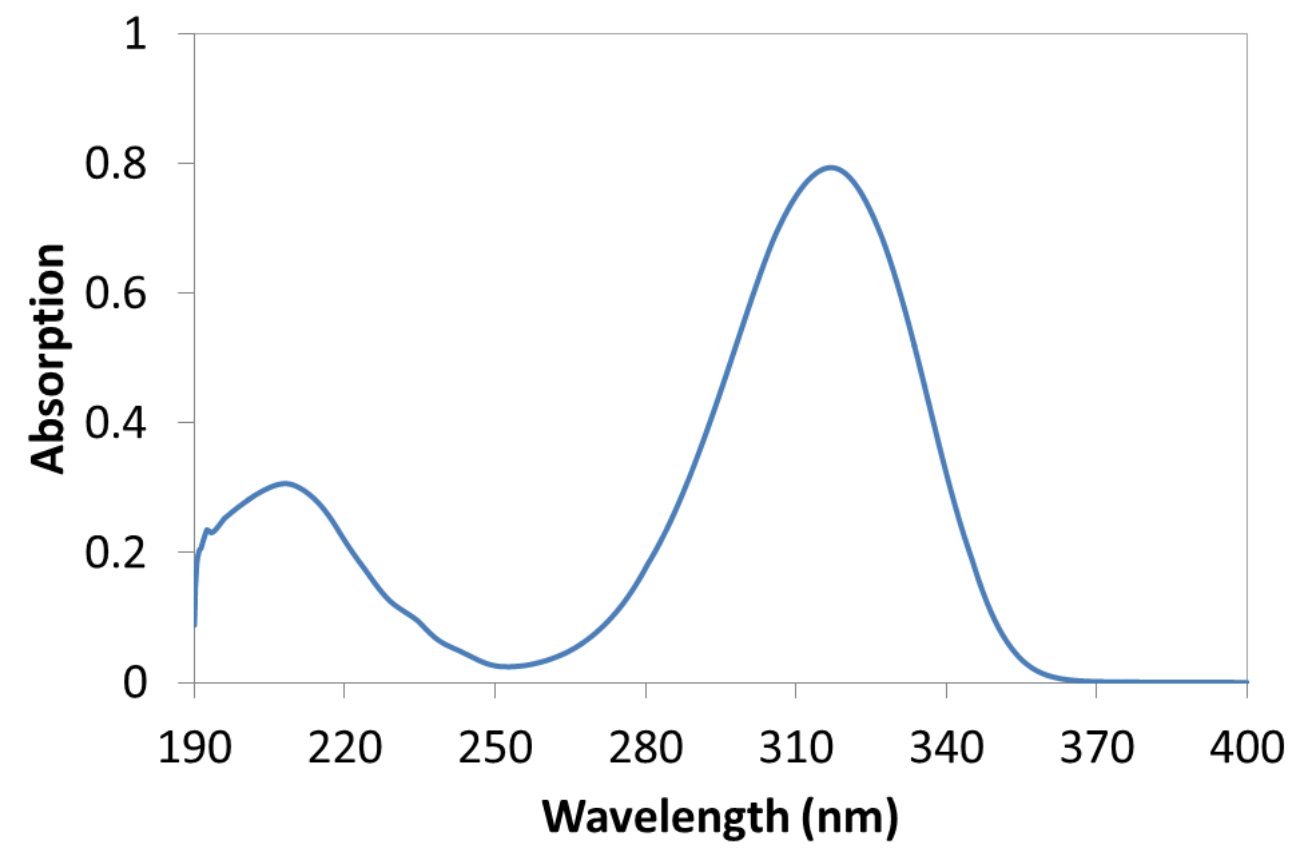


Figure S155. ECD curves of compounds 1-8

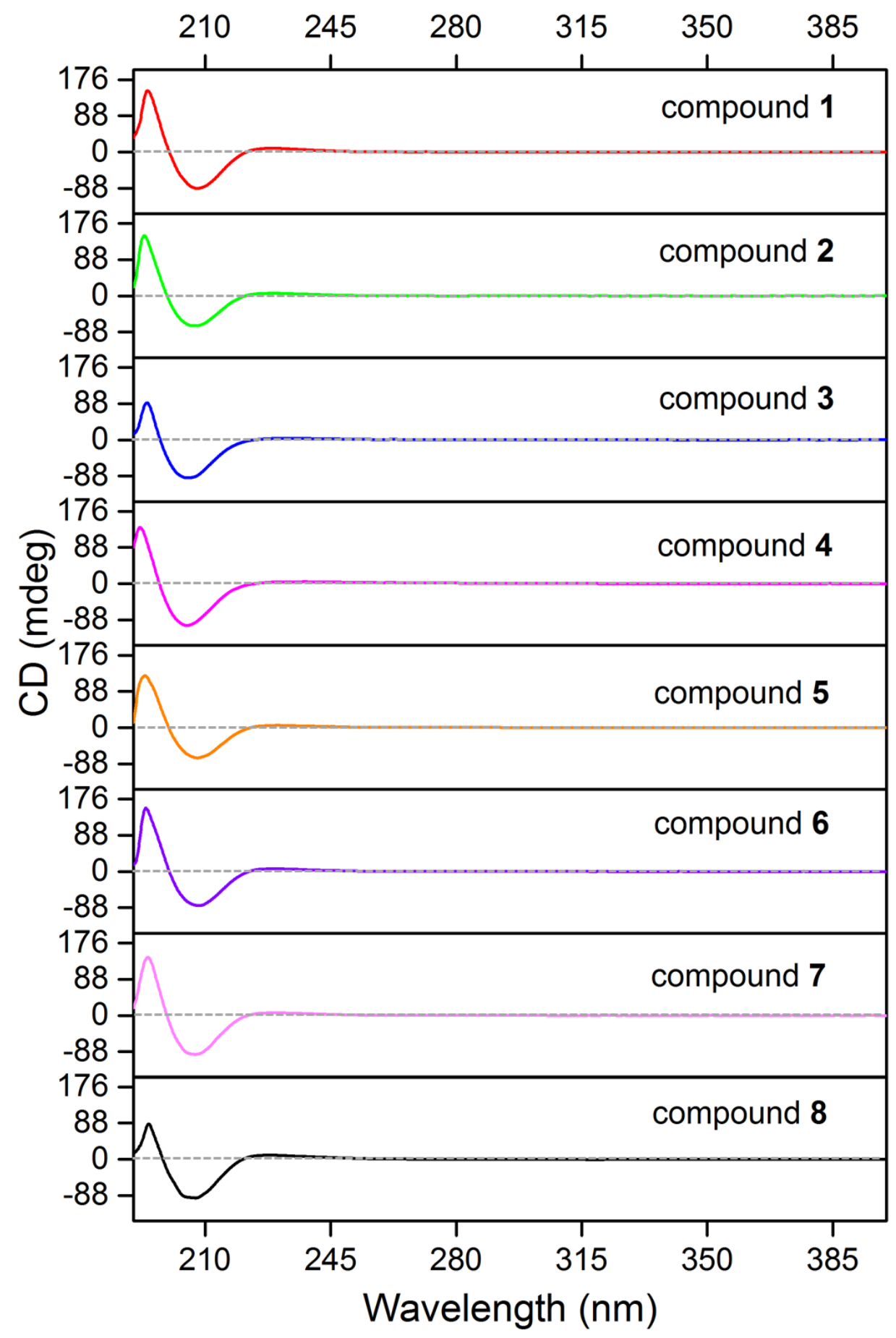


Figure S156. ECD curves of compounds 9-14

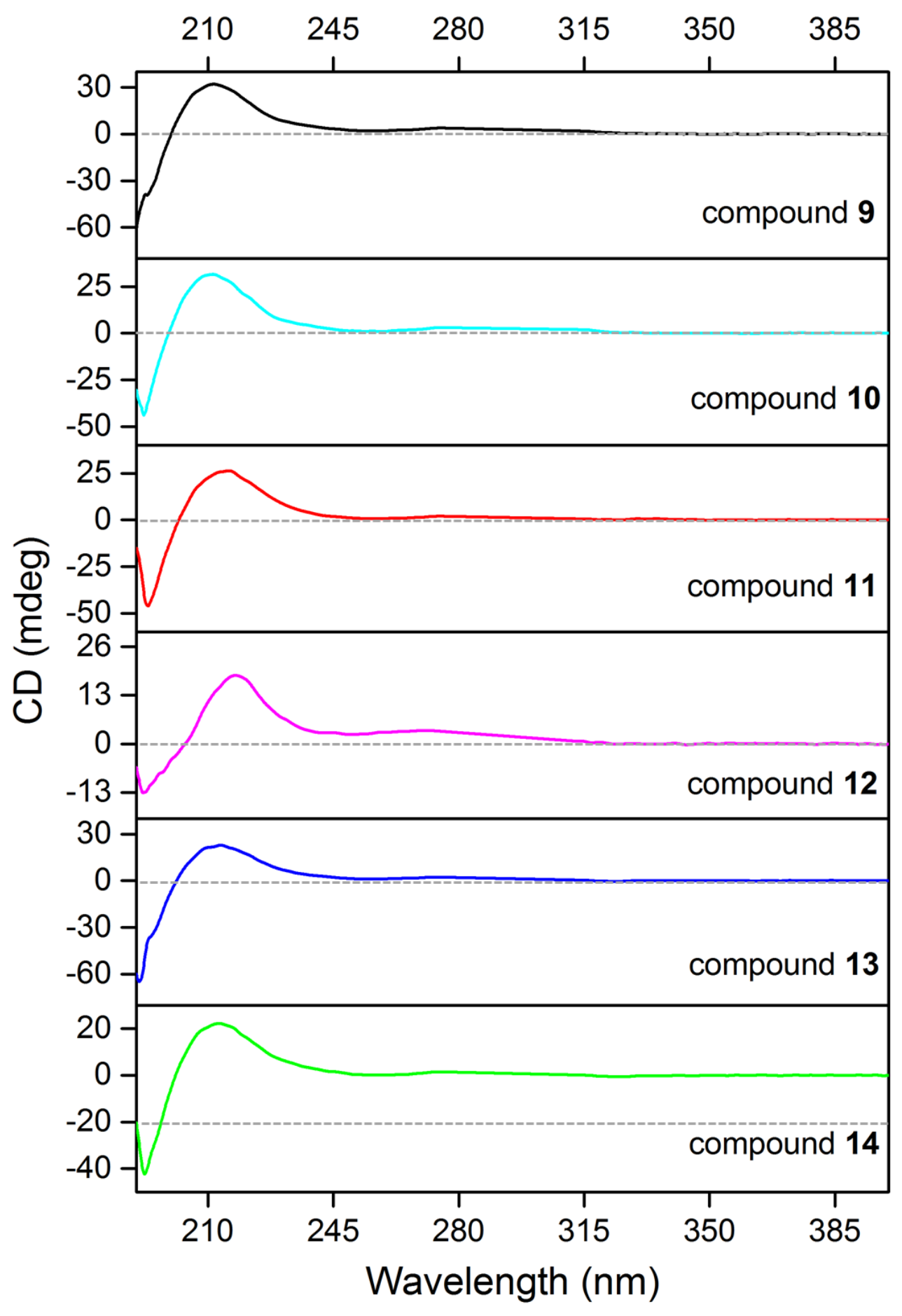


Figure S157. ECD curves of compounds 15-18

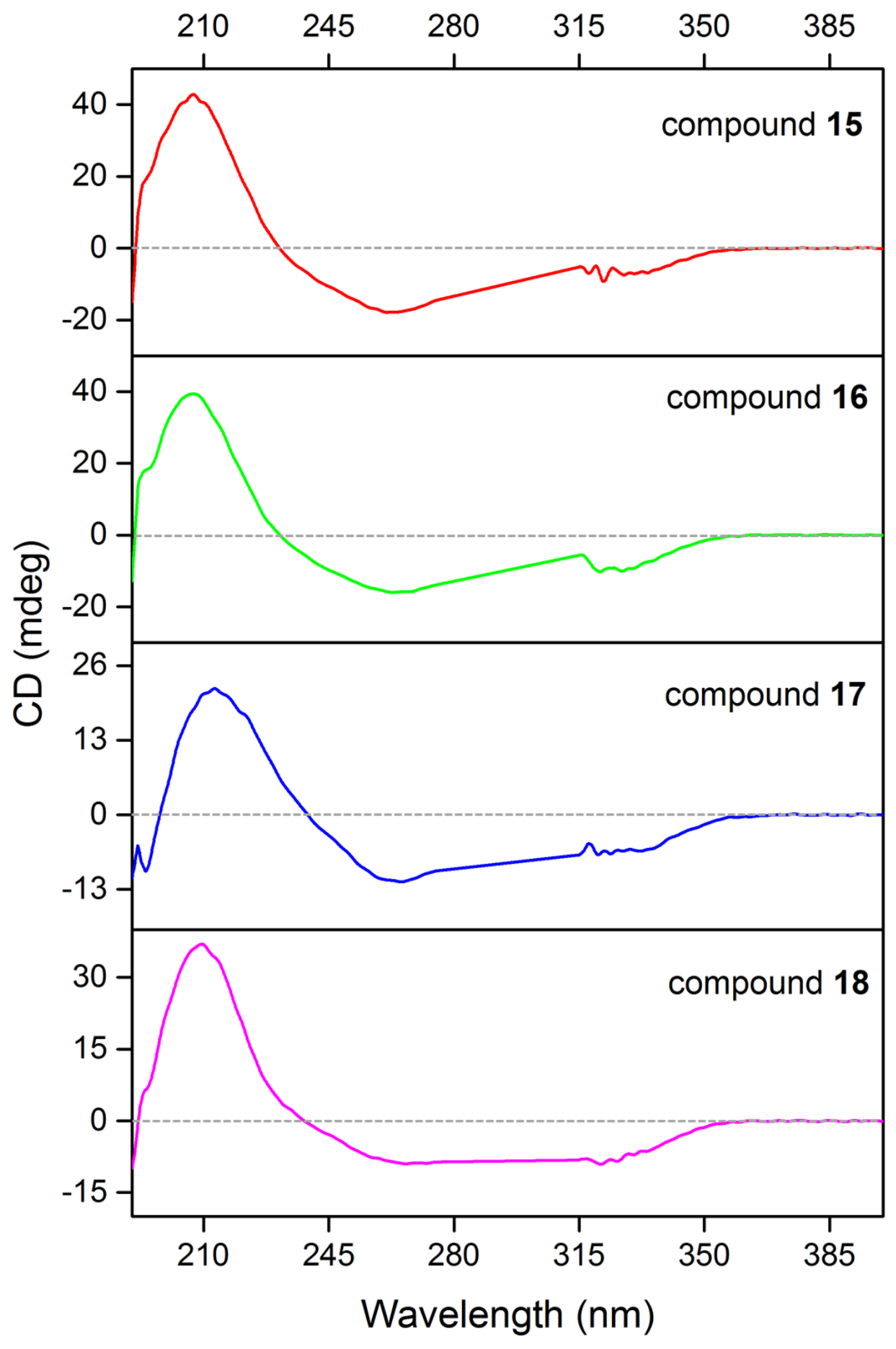


Figure S158. Comparison of the experimental ${ }^{13} \mathrm{C}$ NMR data of compound 9 with the calculated ones

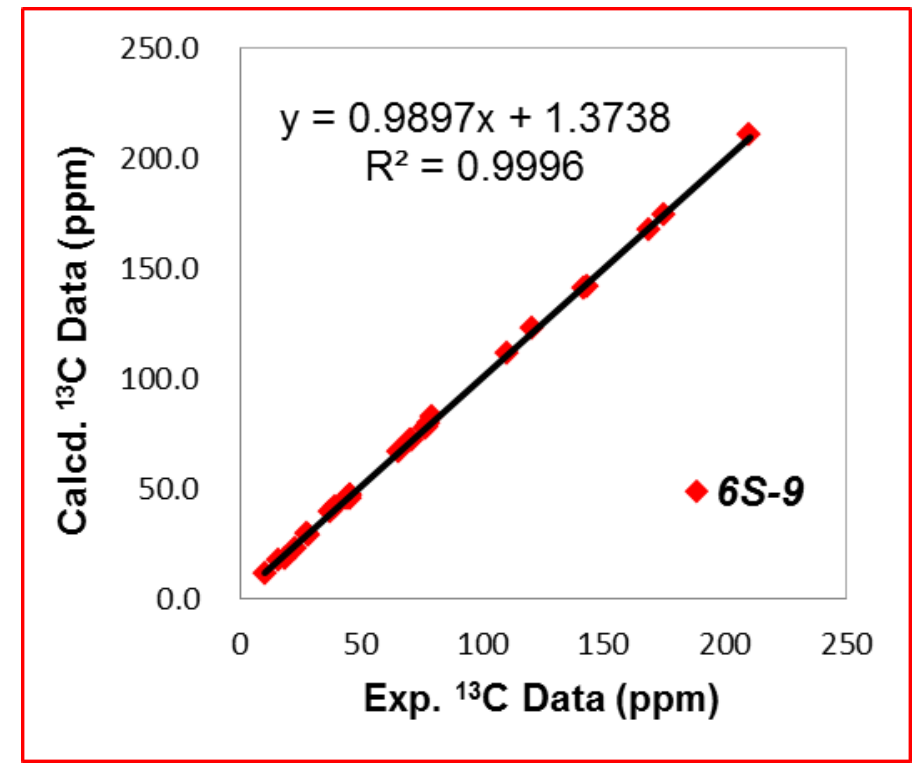


Figure S159. Comparison of the experimental ${ }^{13} \mathrm{C}$ NMR data of compound 11 with the calculated ones

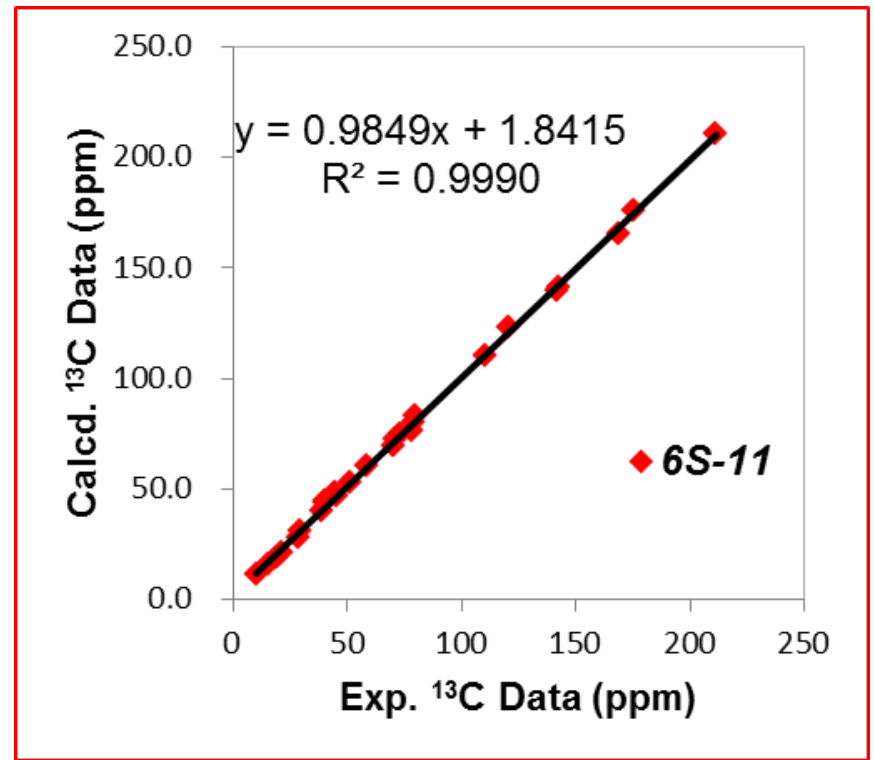


Figure S160. Comparison of the experimental ${ }^{13} \mathrm{C}$ NMR data of compound 18 with the calculated ones

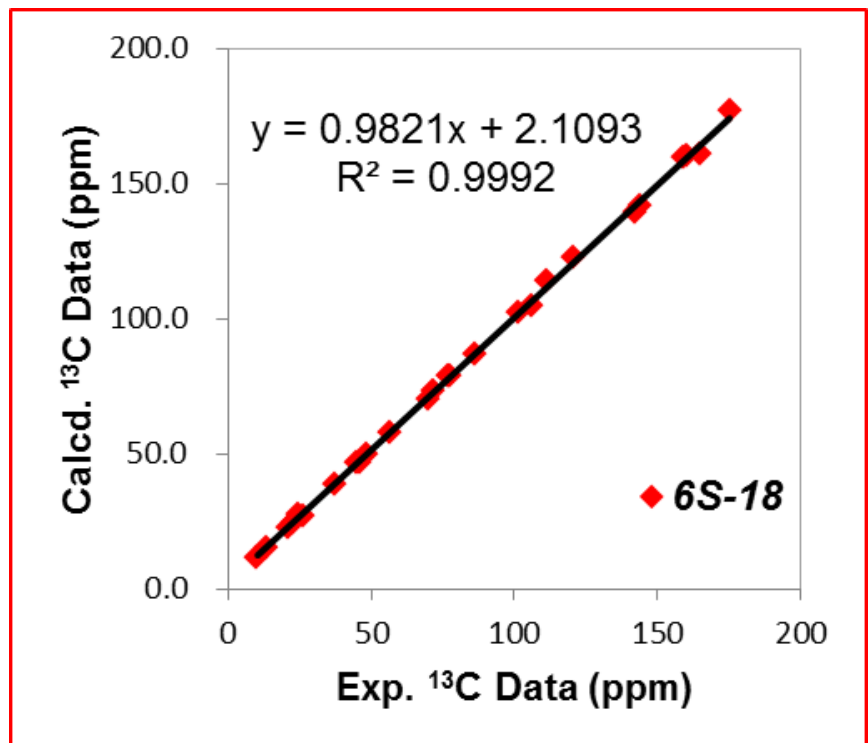

